\title{
AFGMUAN
}

PIBISOMERBS

III

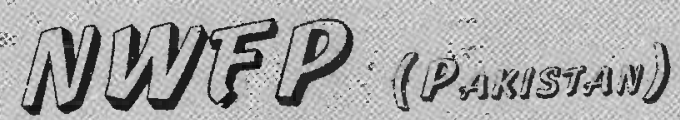

PBISONS

2. 5 U B UEY BEP Q R T
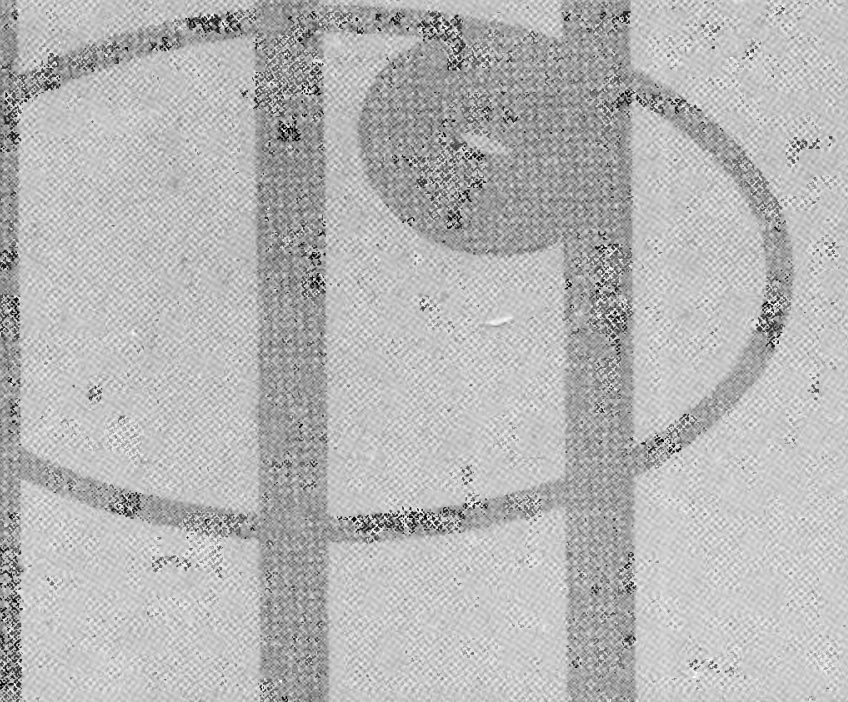

$\frac{x^{2}+\frac{3}{3}}{x^{2}}$

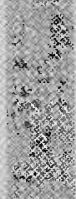

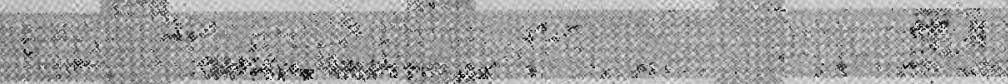

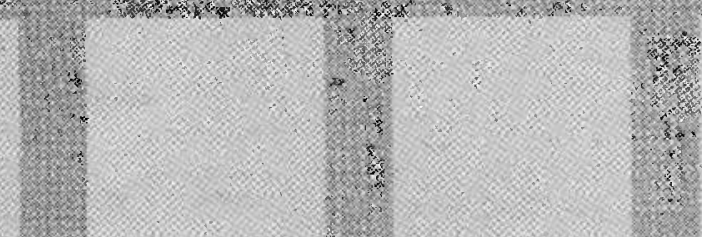




\section{Administered by:}

- Cooperation Centre for Afghanistan (CCA)

- Human Rights Commission of Pakistan (HRCP)

$$
\text { July/December, } 1996
$$

\section{Published by:}

$\rightarrow \quad$ Cooperation Centre for Afghanistan (CCA)

in Peshawar, Pakistan; February, 1997

\section{CCA Office in Bamyan}

420, St. No. 13, E/3, Phase 1. Hayatabad, Peshawar, Pakistan Tel/Fax: $092-0521-810116$ GPO Box No. 1378
Old Bazaar,

Bamyan City, Bamyan,

Afghanistan

\section{CCA Orfice in Mazar:}

Kuch-e-Kalantar, Sarak-e-Bukhdi. Mazar-e-Sharif. Balkh. Afghanistan 
No. Title

Page

1. Preface

2. Introduction

3. Importance of the Survey

4. Objectives of the Survey

5. Procedure

6. List of surveyed prisons

7. Table shows prisons vs number of Afghan refugee prisoners in NWFP

8. District Prison Mardan

9. Judicial Lock up Swabi

10. District Prison Daggar

11. District Prison Swat

12. District Prison Charsadda

13. Central Prison Peshawar

14. District Prison Tairmargara

15. Central Prison Haripur

16. District Prison Kohat

17. District Prison Bannu

18. Central Prison Dara Ismail Khan

19. Judicial Lockup Bajaur

20. Judicial Lockup Dir (upper)

21. Conclusions

22. Crinies Sections 


\section{PREFACE:}

Many Afghans had to leave their country following the invasion of Afghanistan by the Soviet troops in 1979. The numbers grew quickly. According to Pakistan Government 70 per cent of Afghan refugee population in Pakistan have been living in North west Frontier Provinces (NWFP) of Pakistan.

While the nature of the conflict has changed, conflict has not ended. Most refugees cite the continuing hostilities and insecurity as the main reasons for their reluctance to repatriate. Other obstacles are the lack of a recognised authority capable of maintaining law and order. The presence of mines, the destruction of physical structure of the country and the deficiency of food stocks, health care facilities and other public services.

Afghan refugees living in an asylum country are confronted with many problems particularly security problem. The police regard them with suspicion, check their movement and arrest them for minor or ordinary faults. So, beside criminals there are many innocent refugees who are arrested by the police and are kept in the prison. These prisoners should be supported and should be provided with legal aid in order to be released.

CCA as a dedicated human rights organisation has put several steps towards this end. These steps include a survey which was done in collaboration with members of the Human Rights Commission of Pakistan over a period of six months (July 1996 to September 1996) in NWFP of Pakistan. Particulars of around 300 Afghan refugee prisoners were collected and documented during the survey which are, here in, reported and publicised.

It is note-worthy to be mentioned that CCA and HRCP members did the survey voluntarily. CCA has not received any special fund for this project. I would also like to mention that beside our work for identifying the prisoners, we have been trying through independent advocates and contact with authorities for the release of these prisoners. Fortunately, our efforts led to the release of almost one third of these prisoners and work for the release of the remainders are underway.

Publishing this report, CCA, calls upon all concerned organisations like UNHCR, the Governments of Afghanistan and Pakistan as well as Afghan NGOs to pay due attention to and play their expected role in the process of releasing of these prisoners and making the environment safer for the Afghan refugees living in abroad. 


\section{Introduction:}

Afghanistan is a broken country and the citizen of the country persist the weight of different fights attacks in the last 17 year. The ratio of the Afghan refugee people was much higher in the neighbour countries. Now they are living a deplorable and deformed condition, depending deviously on other. Afghanistan is a completed disastrous country and discordant situation also causing discomfort for the Afghan people who have been waiting for the last 17 years for the freedom and peace, It is true that after. the evacuation of USSR army, peace was not maintained in Afghanistan and the circumstances were much estranged among the warring factions and feasible federal government was not constituted in Afghanistan.

Transitional and fickle state, in different protectorate provinces were framed according to their own willing by force and started engaging in the conflicts among themselves. Flock of more Afghan began to take refugee in Pakistan due to these skirmishes.

Afghan people are going to complete here two decades as guests, under UNHCR policy for refugee. They merged with the people of Pakistan in each corner.

In Pakistan Afghan were quite prosperous before 1994. When the propinquity of UNHCR announced forbid in future, their predestination became predicated and worse. So the Afghans joined illegitimate activities with help of local indoor persons. The government of Pakistan decided to keep strict view on the action of Afghan people. Some were arrested, but the majority of them were innocent. Hundred Afghan persons children and women have been arrested under the vagrancy act i.e. 109. They are confined in different prisons in NWFP or in other prisons of the country for the last years. Wife, parents and children are waiting for their releasing. But unlucky, they have no legal and human aid, nor the Afghan NGOs have tried for the deliverance of Afghan deject people. 


\section{Importance of the Survey}

Now-a-days Afghan refugees have confronted different problems. Among of them one is the arrestment of innocent people under the vagrancy act or 14 foreigners act. It is true that their is no law for refugees in Pakistan. Ordinance of the 1959 is specified for Indian refugees not for all refugees. So the migrations and rehabilitation of Afghan refugees in Pakistan is under the UNHCR policies. Police arrest the Afghan, register FIR under section 107, 109, 151, 55 and these people are sent to prisons, which causes also over population of the prisons condition. These arrested and confined person have not been provided legal and human aid. So it was obviously felt that an effective steps may be taken for the deliverance of oppressed Afghan by the co-operation of local Afghan NGOs; which are working for the welfare of Afghan refugees.

\section{Objectives of the Survey:}

1. to assess the number of Afghan refugee prisoners in NWFP prisons;

2. to collect bio date, name, father's name, residence, kinds of crimes and status of legal aid;

3. to provide free legal aid to oppressed prisoners for their releasing;

4. and, try to get co-operation humanly, morally and financially for releasing dejected Afghan prisoners from local Afghan NGOs and other related persons.

\section{Procedure}

The survey project has been divided in two phases:

1. Field work:

Visits of the selected prisons in NWFP and collecting bio data of all confined Afghan refugees oppressed persons.

2. Court work:

- Simple cases will be put in courts for release on bail and acquittal.

- Heavy cases will be put in courts for trails.

The survey was conducted by HRCP and CCA activists group. Questionnaires were used, structured for this purpose and additional information were also collected, then these were tabulated. 


\section{List of surveyed prisons:}

\begin{tabular}{|c|c|c|}
\hline No. & Type of Prisons & Location \\
\hline 1 & District Prison & Mardan \\
\hline 2 & Judicial Lockup & Swabi \\
\hline 3 & District Prison & Daggar, Bunair \\
\hline 4 & District Prison & Swat \\
\hline 5 & District Prison & Charsada \\
\hline 6 & Central prison & Peshawar \\
\hline 7 & District Prison & Taimergara \\
\hline 8 & Central prison & Haripur \\
\hline 9 & District Prison & Kohat \\
\hline 10 & District Prison & Bannu \\
\hline 11 & Central prison & DI Khan \\
\hline 12 & Judicial Lockup & Bajaur \\
\hline 13 & Judicial Lockup & Dir \\
\hline
\end{tabular}

Table shows prisons vs number of Afghan refugee prisoners in NWFP

\begin{tabular}{|c|c|c|c|c|}
\hline S. No. & Name of Prisons & Male & Female & Total \\
\hline 1 & Mardan & 002 & 002 & 004 \\
\hline 2 & Swabi & 015 & 000 & 015 \\
\hline 3 & Daggar & 003 & 002 & 005 \\
\hline 4 & Swat & 015 & 000 & 015 \\
\hline 5 & Charsada & 002 & 002 & 004 \\
\hline 6 & Peshawar & 020 & 000 & 020 \\
\hline 7 & Taimargara & 027 & 000 & 027 \\
\hline 8 & Haripur & 014 & 000 & 014 \\
\hline 9 & Kohat & 128 & 000 & 128 \\
\hline 10 & Bannu & 016 & 001 & 017 \\
\hline 11 & DI Khan & 015 & 000 & 015 \\
\hline 12 & Bajaur & 007 & 000 & 007 \\
\hline \multirow[t]{2}{*}{13} & Dir & 023 & 000 & 023 \\
\hline & Total & 287 & 007 & 294 \\
\hline
\end{tabular}




\section{District Prison Mardan}

\begin{tabular}{|c|c|}
\hline S. No. & $01-01-001$ \\
\hline Name & Shah Bannu \\
\hline Wife of & Noorullah \\
\hline Age & 45 \\
\hline Sex & Female \\
\hline Address in Pakistan & Nasir Bagh Camp, Peshawar, Pakistan \\
\hline \multicolumn{2}{|c|}{ Address in Afghanistan } \\
\hline Accusation/Crime & Arrested under section 4 P.O \\
\hline Date arrested & $09 / 01 / 1996$ \\
\hline Prison & Mardan \\
\hline Type of Prison & District \\
\hline Present condition & Not released. \\
\hline Remarks & $\begin{array}{l}\text { She was produced to different courts in Swabi now the court has decided her case, but she is till in the prison. } \\
\text { She said to the team that she was innocent, all the allegations are false. She needed human aid for bail. }\end{array}$ \\
\hline
\end{tabular}




\begin{tabular}{|c|c|}
\hline S. No. & $01-02-002$ \\
\hline Wife of & Murad Ali \\
\hline Age & 25 \\
\hline Sex & Female \\
\hline Accusation/Crime & was charged under section $\mathrm{Z} .0 \mathrm{O} 05 / 10+11$ \\
\hline Date arrested & $25 / 01 / 1996$ \\
\hline Prison & Mardan \\
\hline Type of Prison & District \\
\hline
\end{tabular}

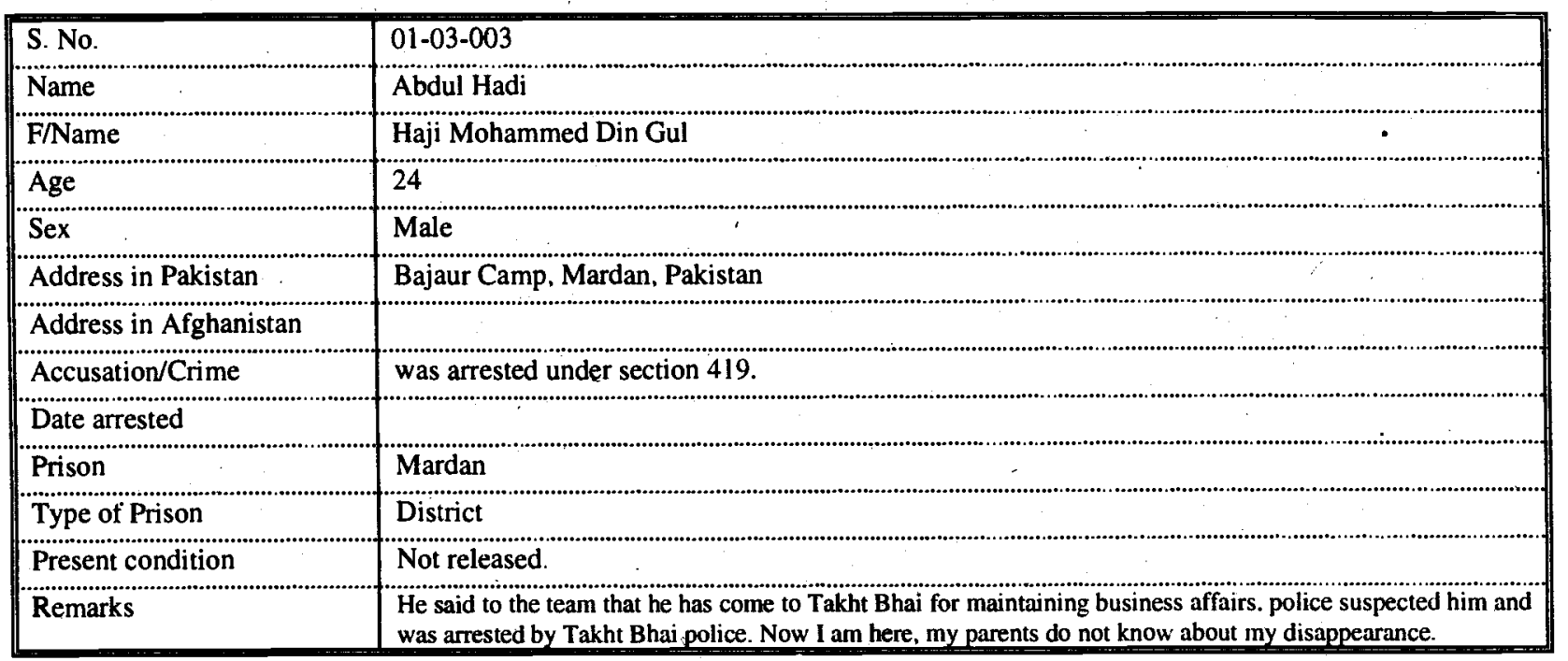

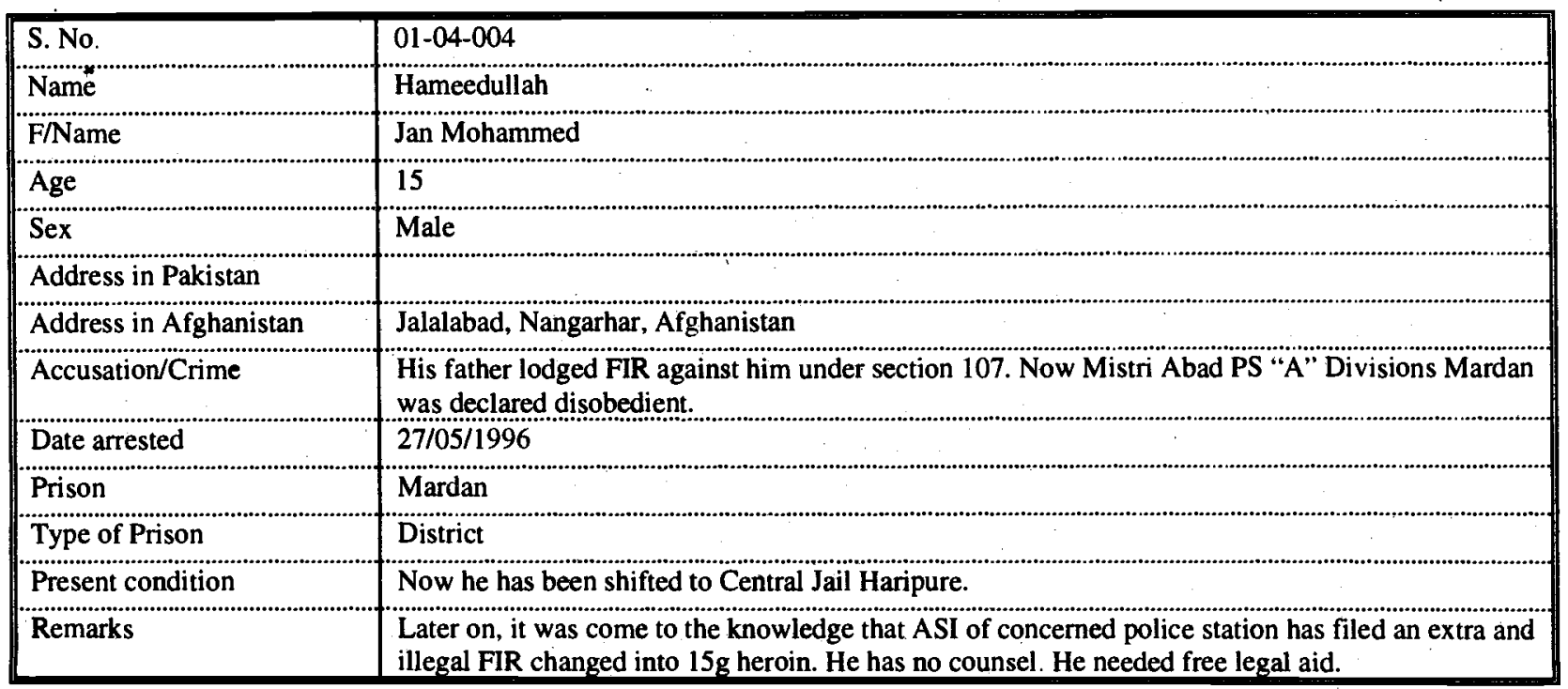




\section{Iudicial Lockup Swabi}

\begin{tabular}{||l|l||}
\hline S. No. & $02-01-005$ \\
\hline Name & lslamuddin \\
\hline F/Name & Sair Aulia \\
\hline Age & 30 \\
\hline Sex & Male \\
\hline Address in Pakistan & Gandaf Camp, Swabi, NWFP, Pakistan \\
\hline Address in Afghanistan & Kunar, Afghanistan \\
\hline Accusation/Crime & was charged under section 379 PPC \\
\hline Date arrested & $12 / 04 / 1996$ \\
\hline Prison & Swabi \\
\hline Type of Prison & Judicial Lockup \\
\hline Present condition & His case in under trial. \\
\hline Remarks & He was arrested by IDS Police. \\
\hline
\end{tabular}




\begin{tabular}{|c|c|}
\hline S. No: & $02-02-006$ \\
\hline Name & Musa Khan \\
\hline F/Name & Redi Gul \\
\hline Age & 25 \\
\hline Sex & Male \\
\hline Address in Pakistan & Barakai Camp, Swabi, NWFP. Pakistan \\
\hline Address in Afghanistan & Logar. Afghanistan \\
\hline Accusation/Crime & was arrested under section 420,419 . \\
\hline Date arrested & $18 / 04 / 1996$ \\
\hline Prison & Swabi \\
\hline Type of Prison & Judicial Lockup \\
\hline Present condition & He was released on $05 / 09 / 1996$ after a joint effort of CCA \& HRCP. \\
\hline Remarks & $\begin{array}{l}\text { Once his bail application has been rejected by the session court Swabi, then he has submitted } \\
\text { appeal for bail to Peshawar High Court. }\end{array}$ \\
\hline
\end{tabular}

\begin{tabular}{|c|c|}
\hline S. No. & $02-03-007$ \\
\hline Name & Haroon \\
\hline F/Name & Akhtar Mohammed \\
\hline Age & 19 \\
\hline Sex & Male \\
\hline Address in Pakistan & Barakai Camp, Swabi. Pakistan \\
\hline Address in Afghanistan & Karabagh, Kabul, Afghanistan \\
\hline Accusation/Crime & was arrested under section 419,420 . \\
\hline Date arrested & $19 / 04 / 1996$ \\
\hline Prison & Swabi \\
\hline Type of Prison & Judicial Lockup \\
\hline Present condition & He was released on $05 / 09 / 1996$ after a joint effort of CCA \& HRCP. \\
\hline Remarks & His bail application had once rejected by the Session Court. \\
\hline
\end{tabular}

\begin{tabular}{|c|c|}
\hline S. No. & 02-04-008 \\
\hline Name & Lal Mohammed \\
\hline F/Name & Mohammed Qibad \\
\hline Age & 28 \\
\hline Sex & Male \\
\hline Address in Pakistan & Barakai Camp, Swabi, NWFP, Pakistan \\
\hline Address in Afghanistan & Karabagh, Kabul, Afghanistan \\
\hline Accusation/Crime & was charged under section 334 . \\
\hline Date arrested & $12 / 04 / 1996$ \\
\hline Prison & Swabi \\
\hline Type of Prison & Judicial Lockup \\
\hline Present condition & He was released on $05 / 09 / 1996$ after a joint effort of CCA \& HRCP. \\
\hline Remarks & $\begin{array}{l}\text { He said that his case had been delaying due to not produce witnesses. The man who had blamed to me also } \\
\text { makes ostences. The court changed the dates for further procedure for the last three months. }\end{array}$ \\
\hline
\end{tabular}




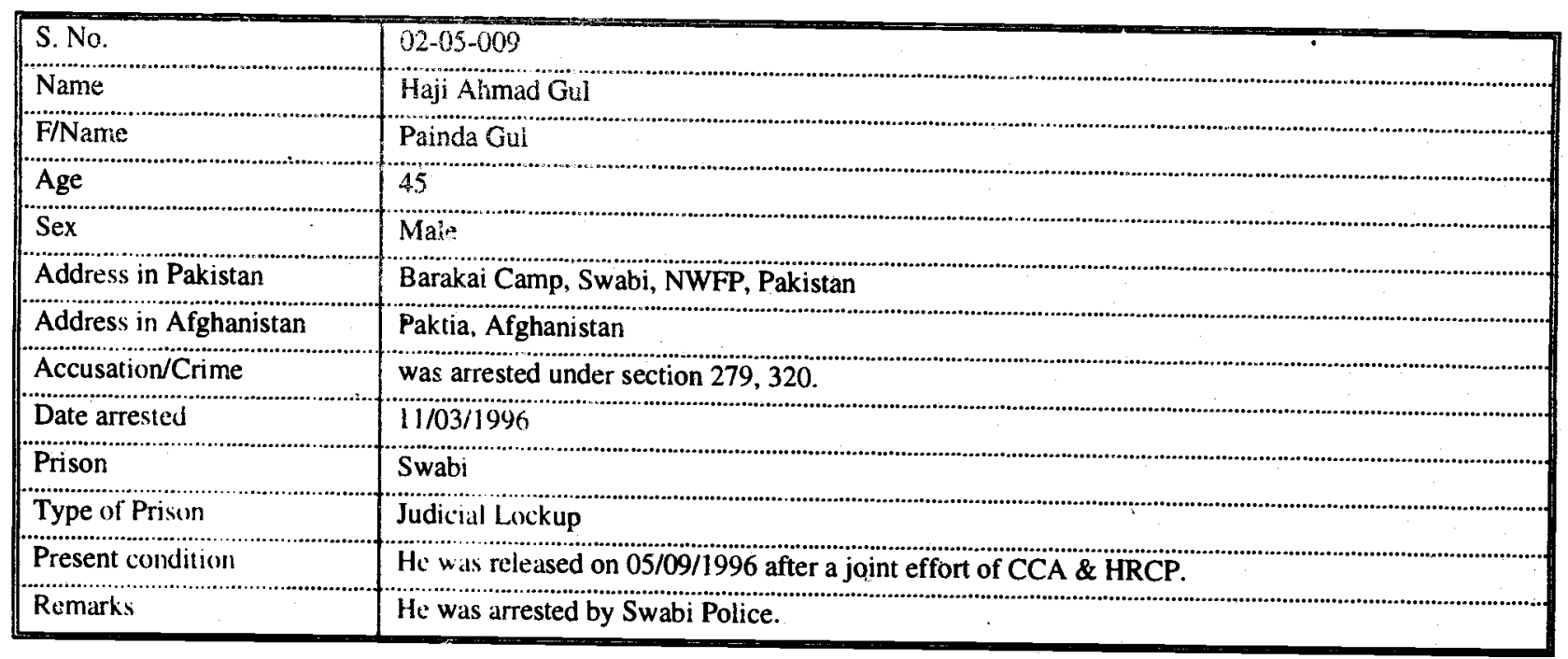

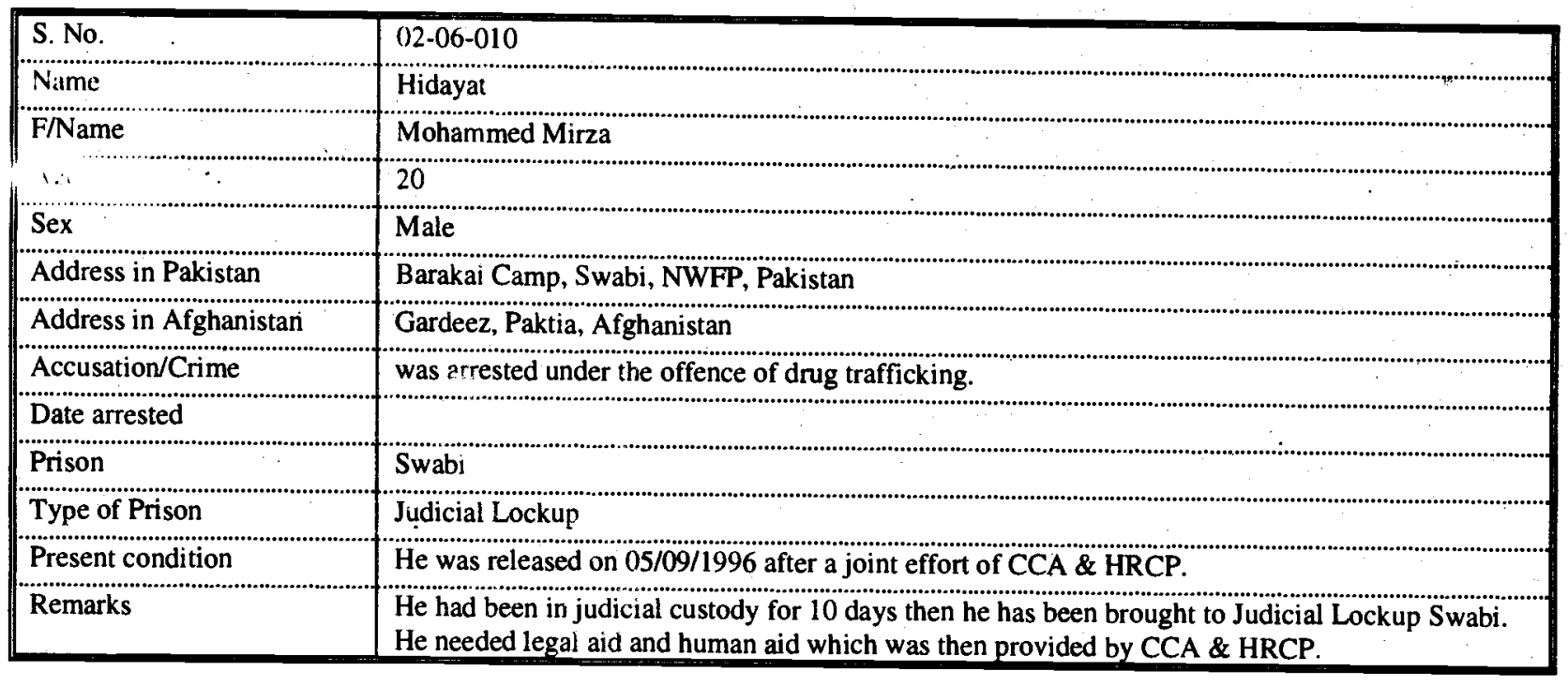

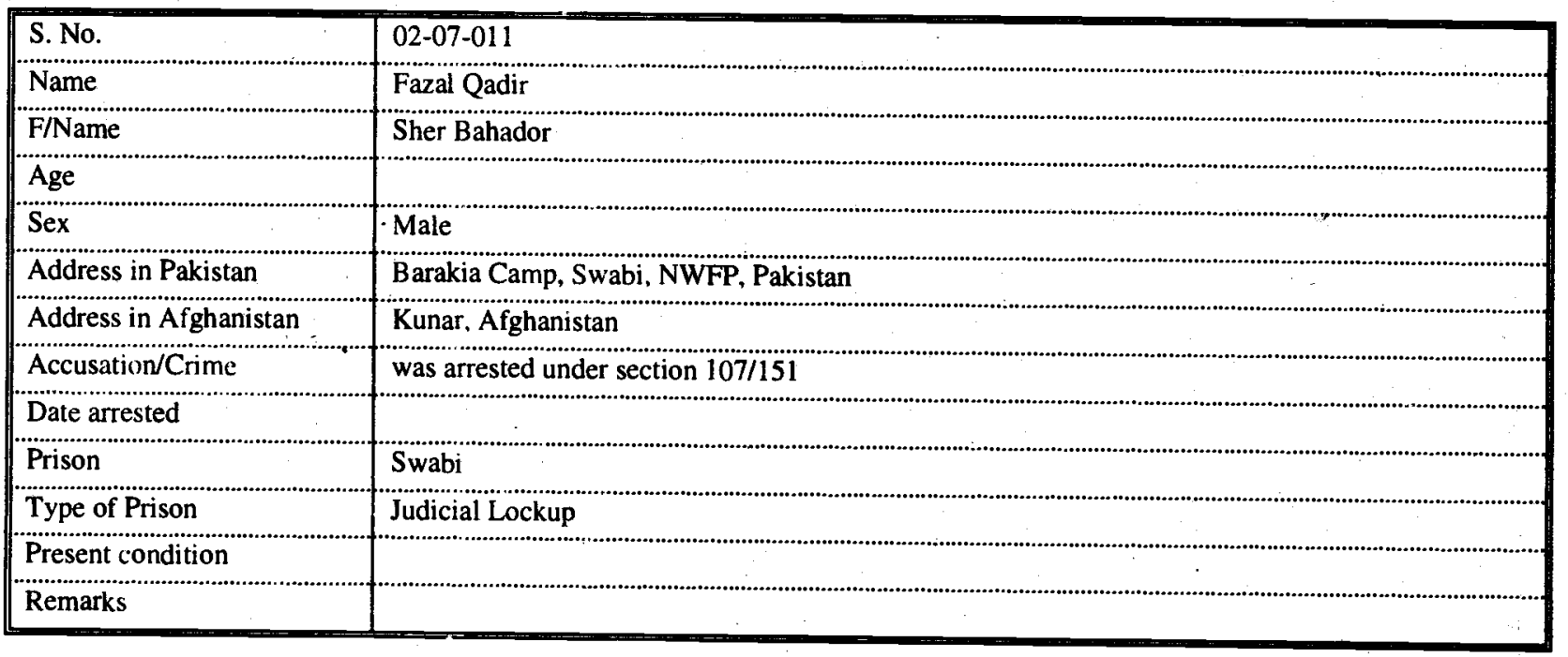




\begin{tabular}{|c|c|}
\hline S. No. & $02-08-012$ \\
\hline Name & Salehddin \\
\hline F/Name & Said Ulia \\
\hline Age & . \\
\hline Sex & Male \\
\hline Address in Pakistan & Barakai, Swabi, NWFP, Pakistan \\
\hline Accusation/Crime & was arrested under section $107 / 151$ \\
\hline \multicolumn{2}{|l|}{ Date arrested } \\
\hline Prison & Swabi \\
\hline Type of Prison & Judicial Lockup \\
\hline
\end{tabular}

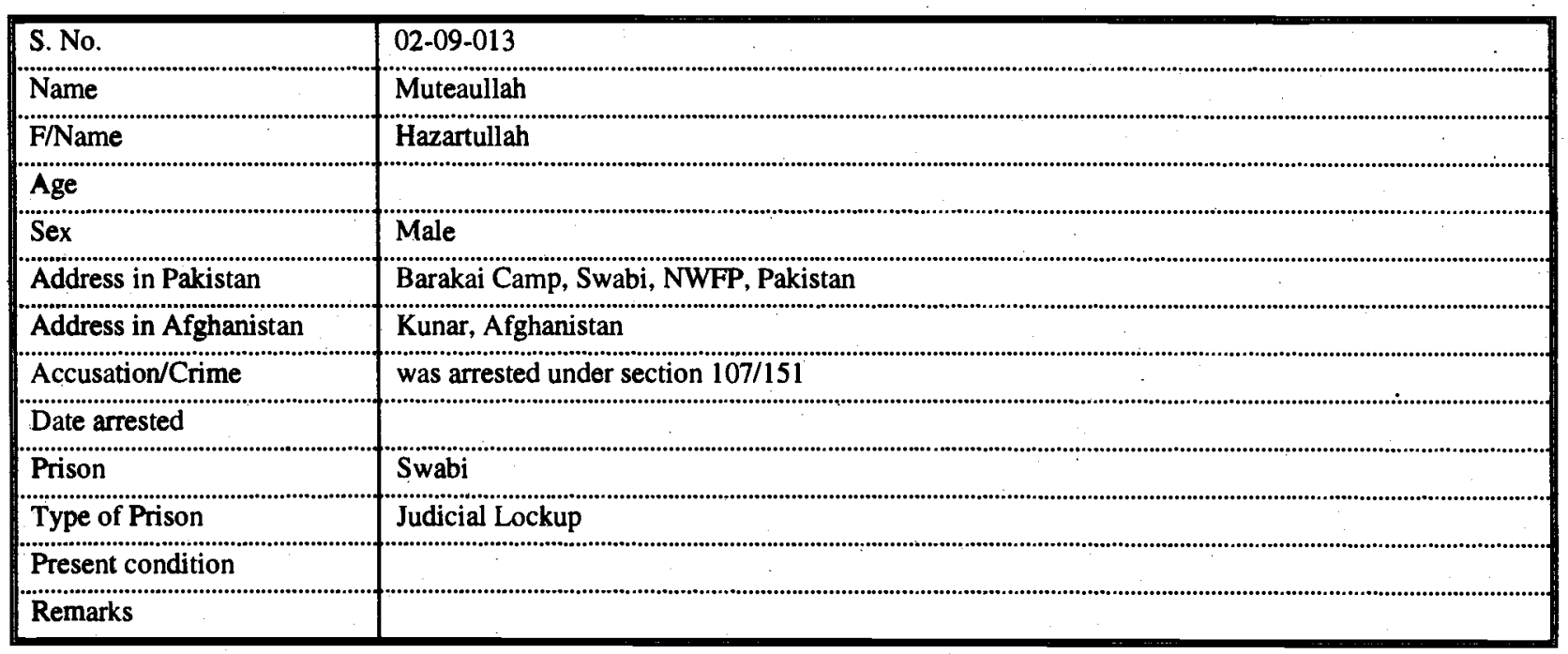

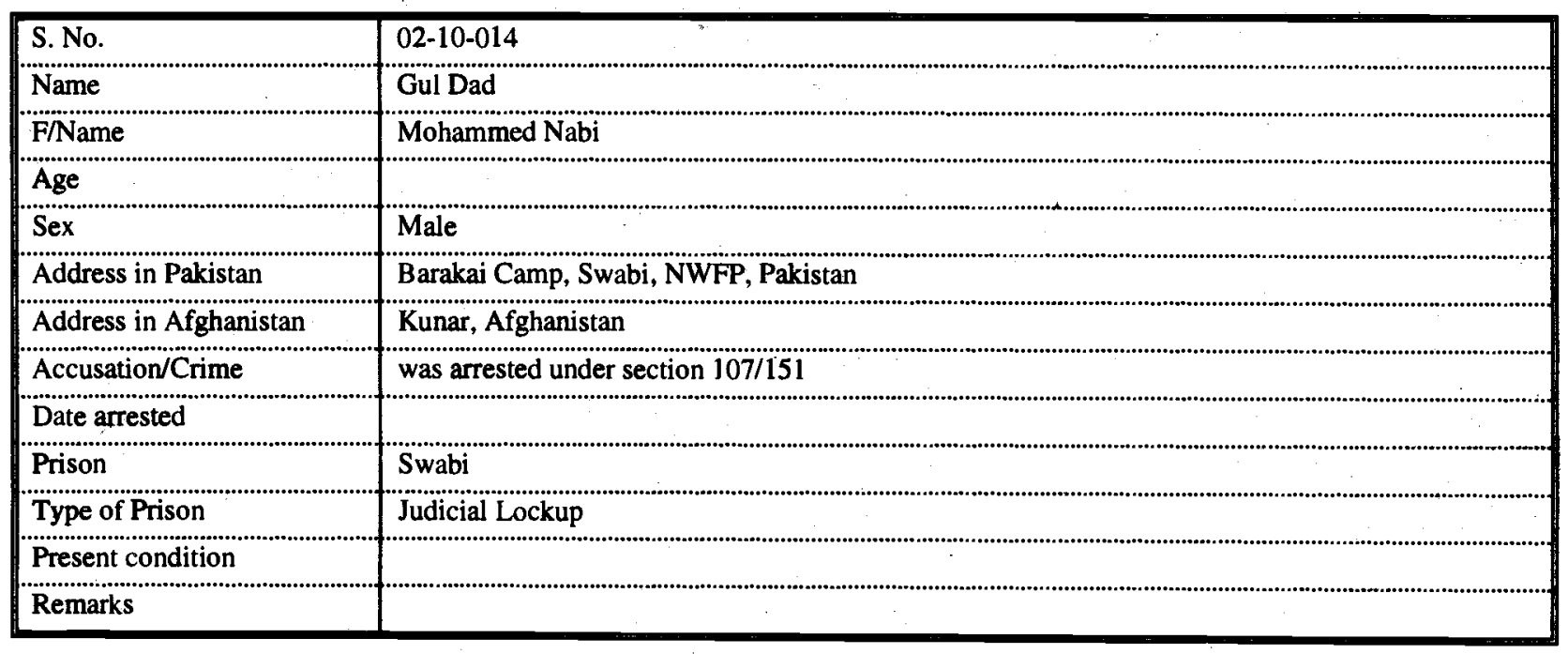




\begin{tabular}{|c|c|}
\hline S. No. & $02-11-015$ \\
\hline Name & Sayyed Ghani \\
\hline F/Name & Abdul Ghani \\
\hline \multicolumn{2}{|l|}{ Age } \\
\hline Sex & Male \\
\hline Accusation/Crime & was arrested under section $107 / 151$ \\
\hline \multicolumn{2}{|l|}{ Date arrested } \\
\hline Prison & Swabi \\
\hline Type of Prison & Judicial Lockup \\
\hline
\end{tabular}

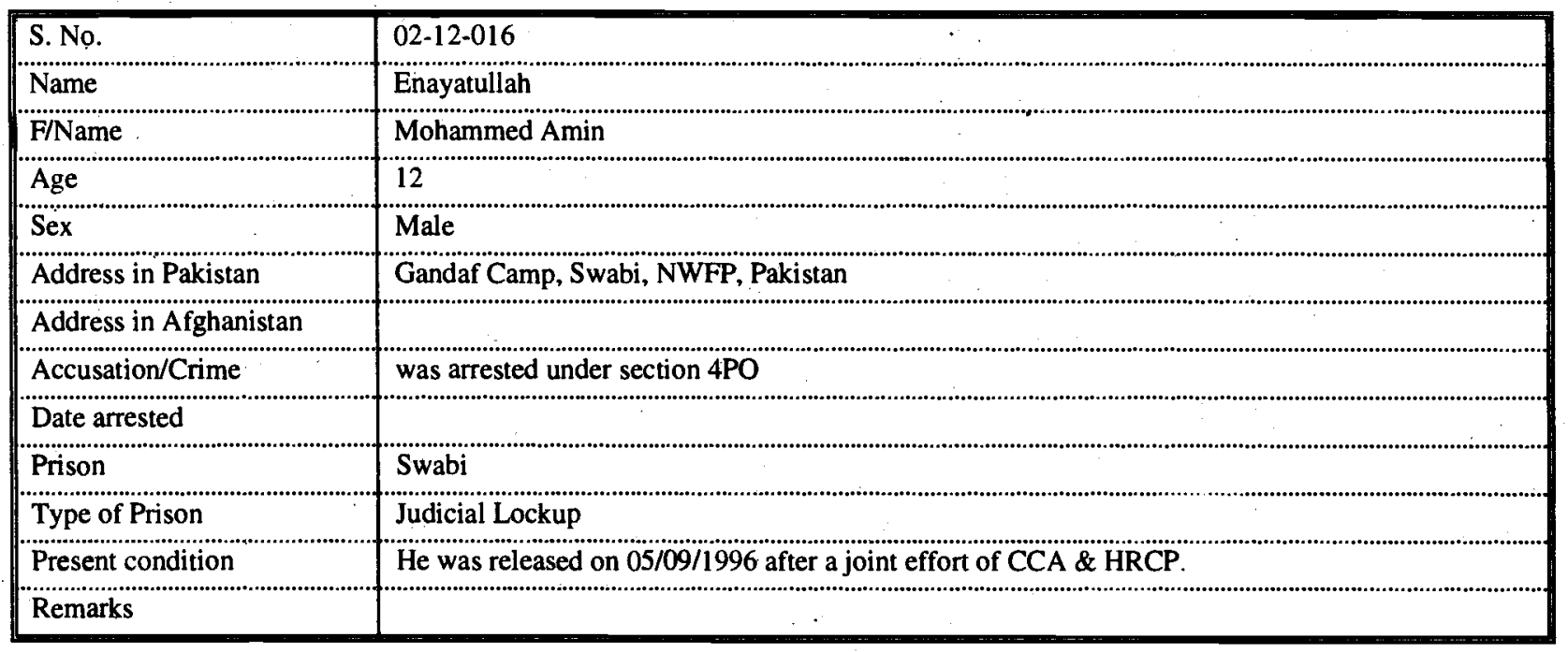

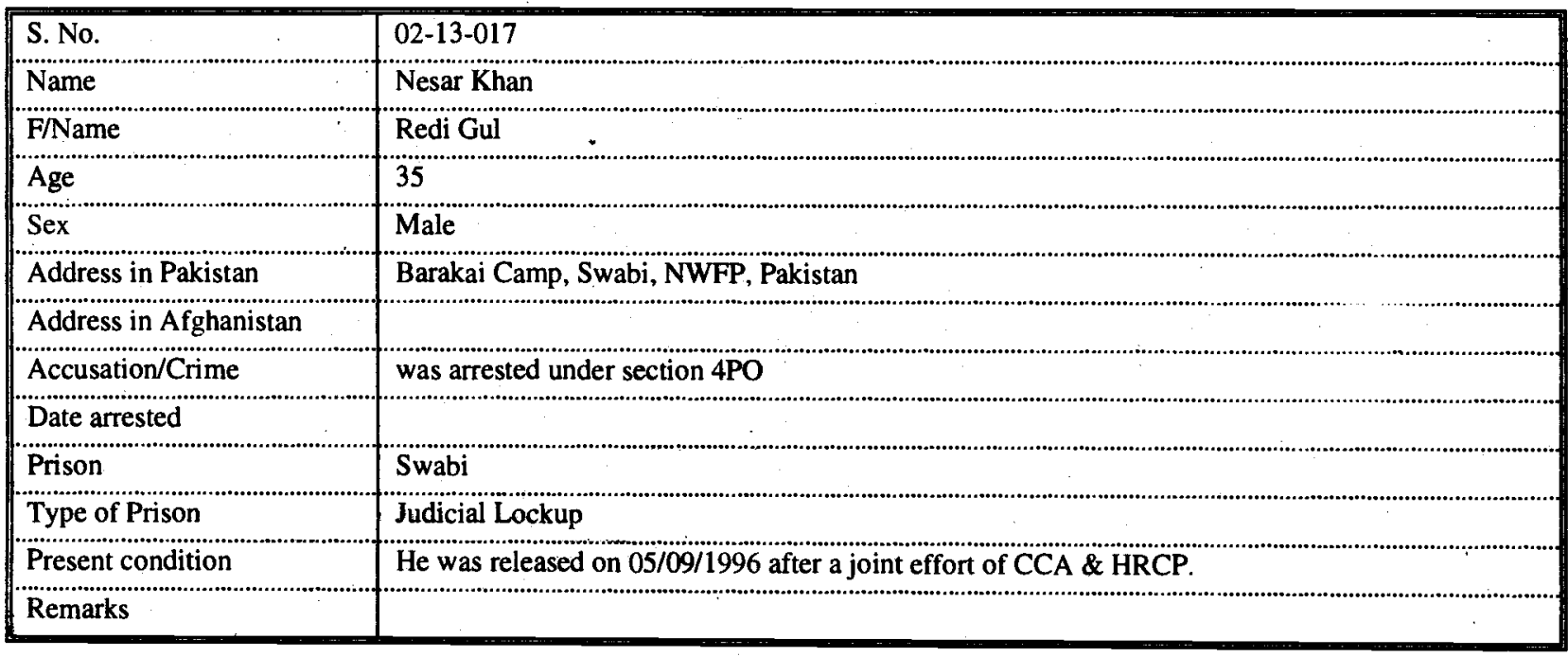




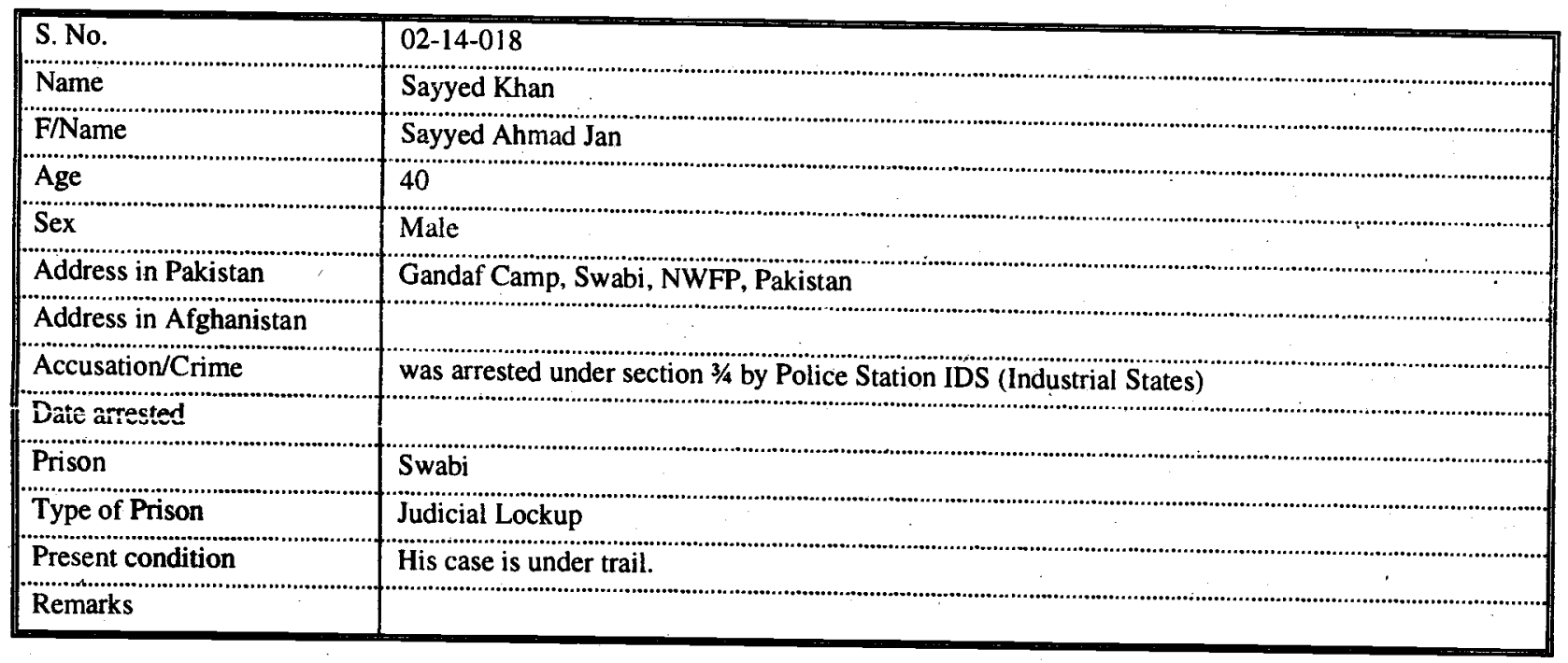

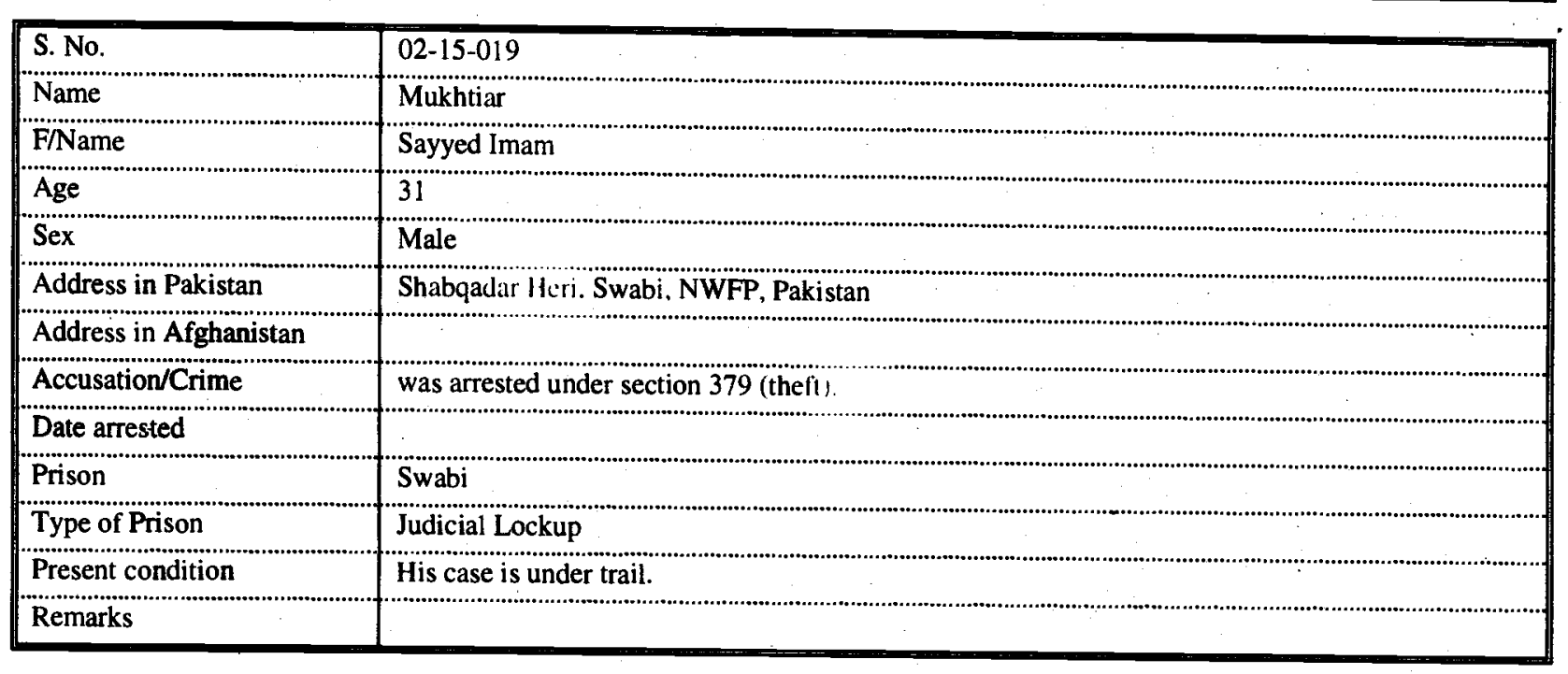




\section{District Prison Daggar}

\begin{tabular}{|c|c|}
\hline S. No. & $03-01-020$ \\
\hline Namé & Abdul Mateen \\
\hline F/Name & Abdul Waheed \\
\hline Age & 26 \\
\hline Sex & Male \\
\hline Address in Pakistan & Koga Camp, Bunair, Daggar, NWFP, Pakistan \\
\hline \multicolumn{2}{|c|}{ Address in Afghanistan } \\
\hline Accusation/Crime & was arrested under section 302 . \\
\hline Date arrested & $12 / 02 / 1996$ \\
\hline Prison & Bunair, Daggar District \\
\hline Type of Prison & District. \\
\hline Present condition & He was released on $26 / 08 / 1996$ after a joint effort of CCA \& HRCP. \\
\hline Remarks & $\begin{array}{l}\text { His father Abdul Waheed registered against hin killing brother's wife and he was brought to totally police } \\
\text { station. His cuse had not been conmenced nor the counsel was available for him for sometimes. }\end{array}$ \\
\hline
\end{tabular}




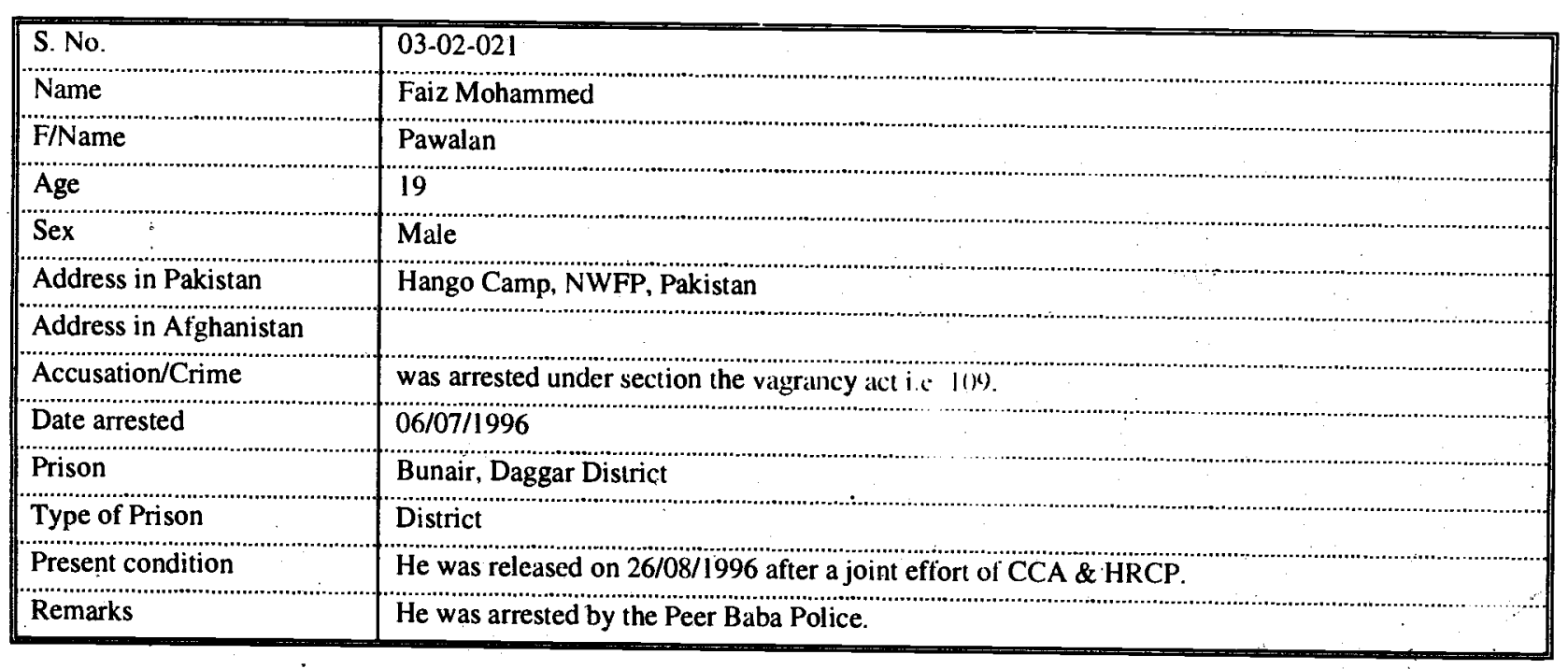

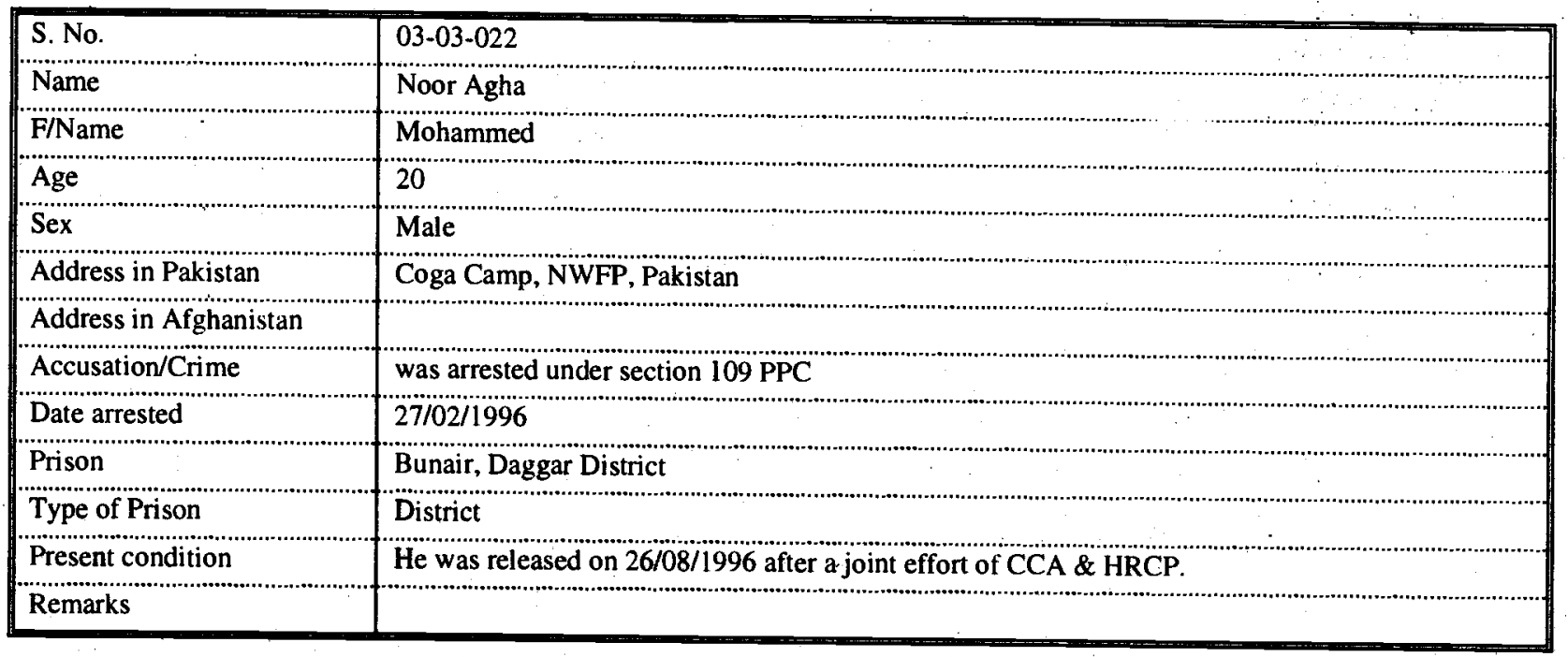

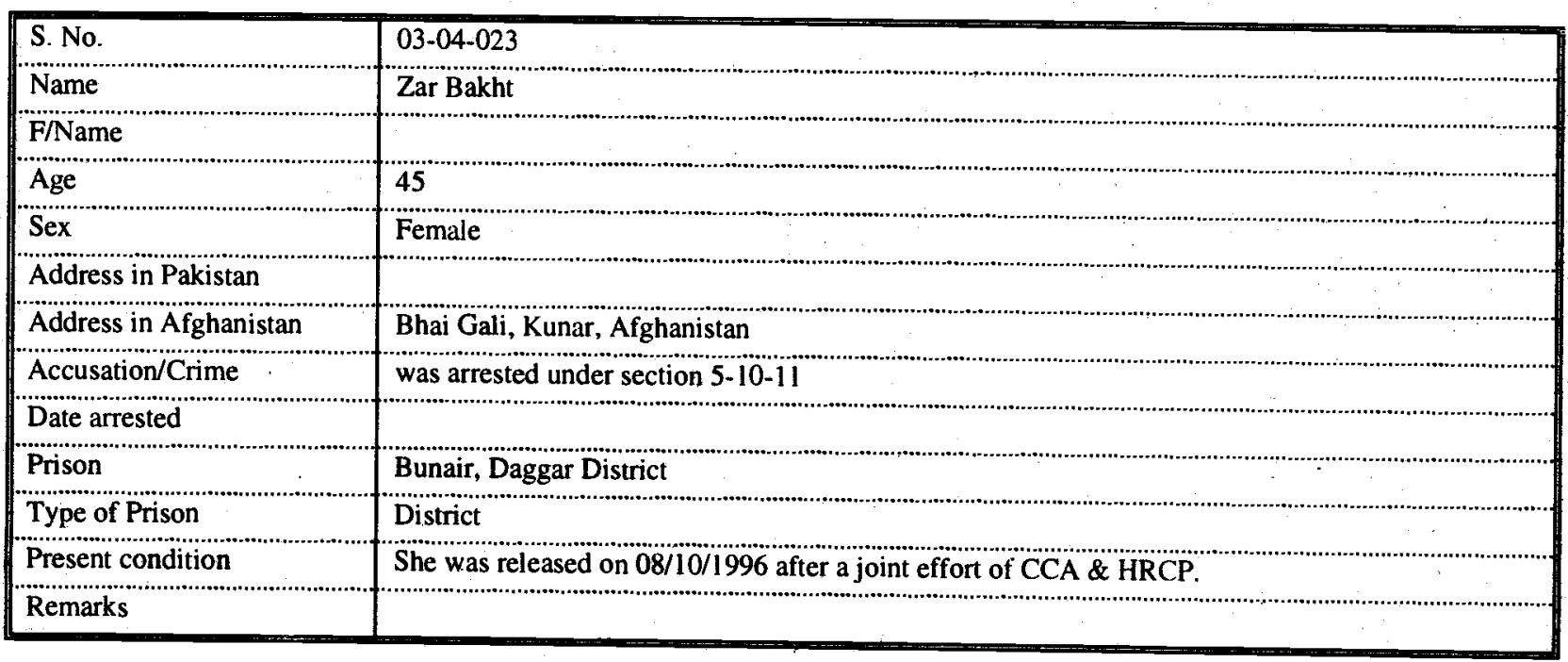




\begin{tabular}{|c|c|}
\hline S. No. & $03-05-024$ \\
\hline Name & Rafiqa \\
\hline \multicolumn{2}{|l|}{ F/Name } \\
\hline Age & $45 \cdot$ \\
\hline Sex & Female \\
\hline \multicolumn{2}{|l|}{ Address in Pakistan } \\
\hline Address in Afghanistan & Bhai Gali. Kunar, Afghanistan \\
\hline Accusation/Crime & was arrested under section $5-10-11$ \\
\hline \multicolumn{2}{|l|}{ Date arrested } \\
\hline Prison & Bunair, Daggar District \\
\hline Type of Prison & District \\
\hline Present condition & He was released on 08/10/1996 after a joint effort of CCA \& HRCP \\
\hline Remarks & \\
\hline
\end{tabular}




\section{District Prison Swat}

\begin{tabular}{|c|c|}
\hline S. No. & $04-01-025$ \\
\hline Name & Rozi Khan \\
\hline F/Name & Azam Khan \\
\hline Age & 25 \\
\hline Sex & Male \\
\hline Address in Pakistan & Kochi Garhi Camp, Peshawar, NWFP, Pakistan \\
\hline Address in Afghanistan & . \\
\hline Accusation/Crime & was arrested under section vagrancy act 109 . \\
\hline Date arrested & $06 / 02 / 1996$ \\
\hline Prison & Swat \\
\hline Type of Prison & District \\
\hline Present condition & He was released on $25 / 08 / 1996$ after a joint effort of CCA \& HRCP. \\
\hline Remarks & $\begin{array}{l}\text { He was arrested by PS Mingora. He was working at Mingora Bazaar on daily wages basis in a fruit shop. He } \\
\text { said to the team that he has needed legal \& human rights which then was provided by CCA \& HRCP. }\end{array}$ \\
\hline
\end{tabular}




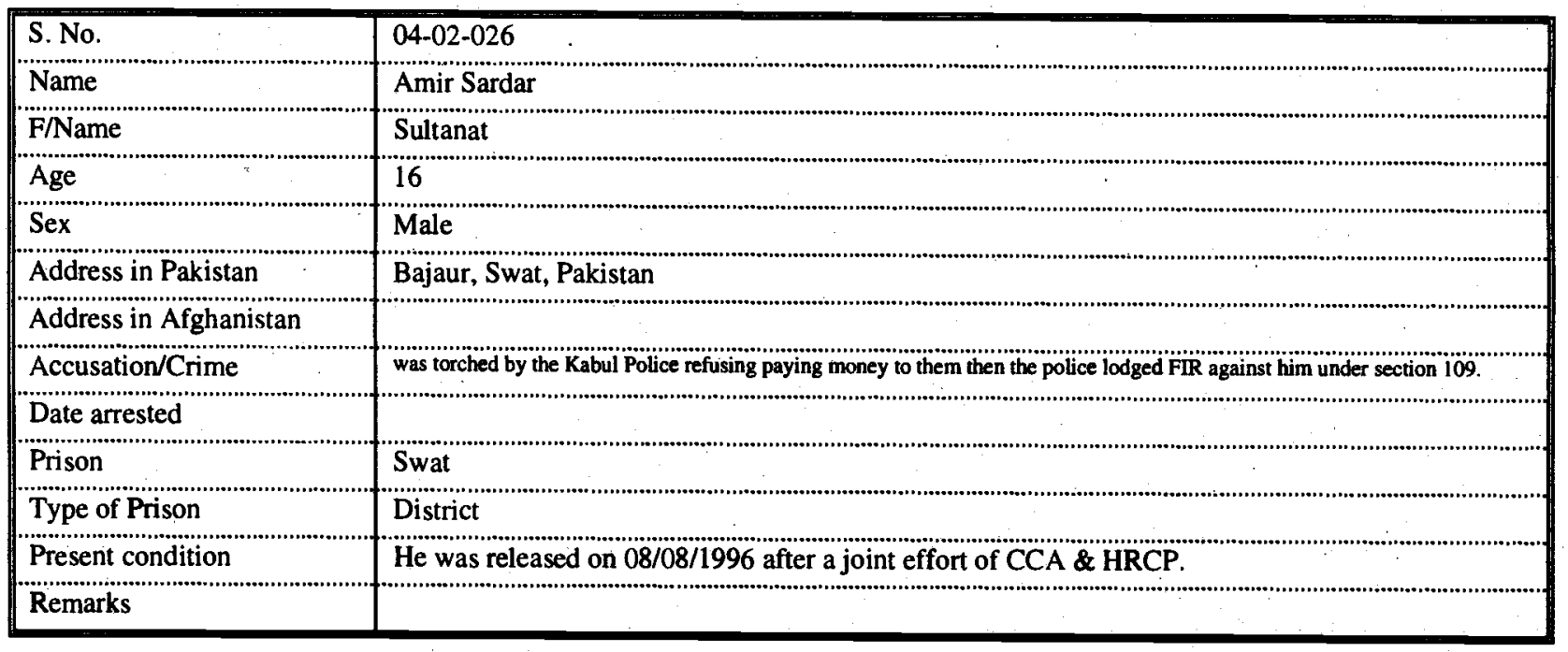

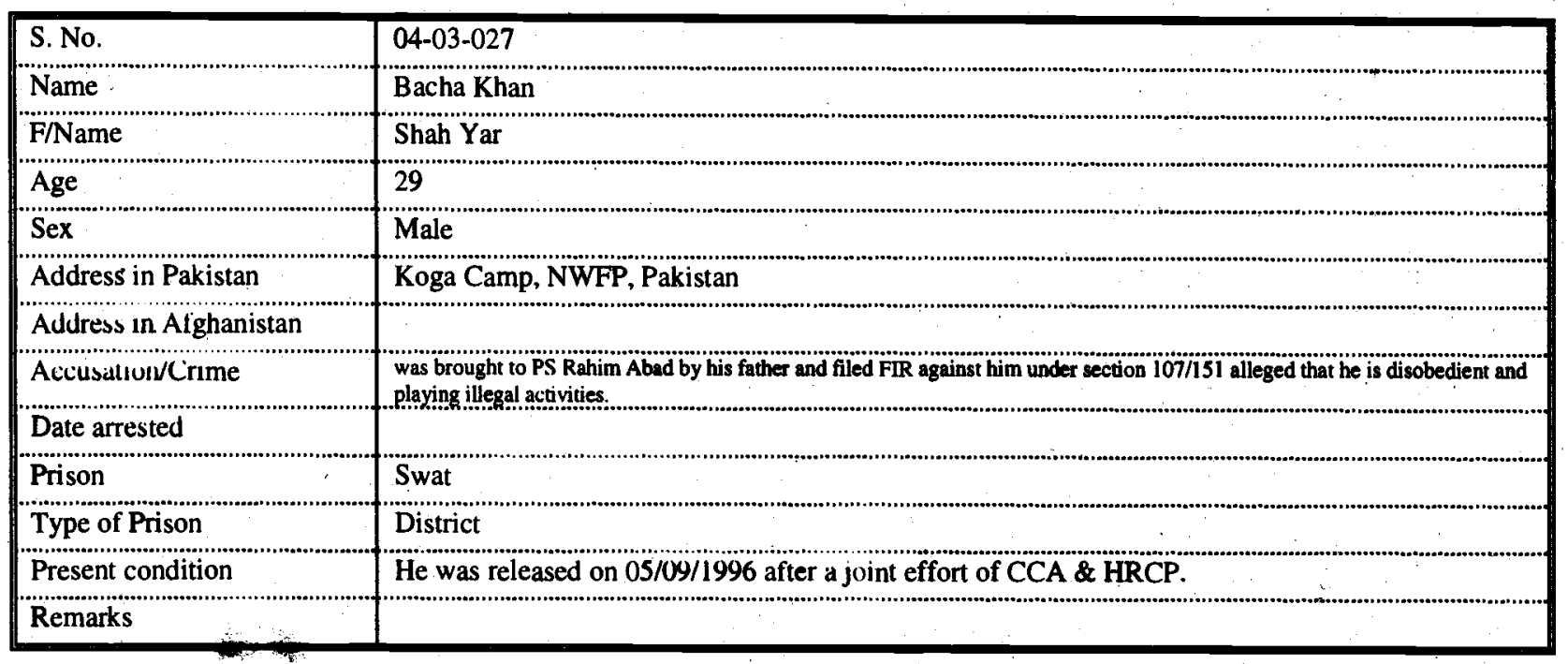

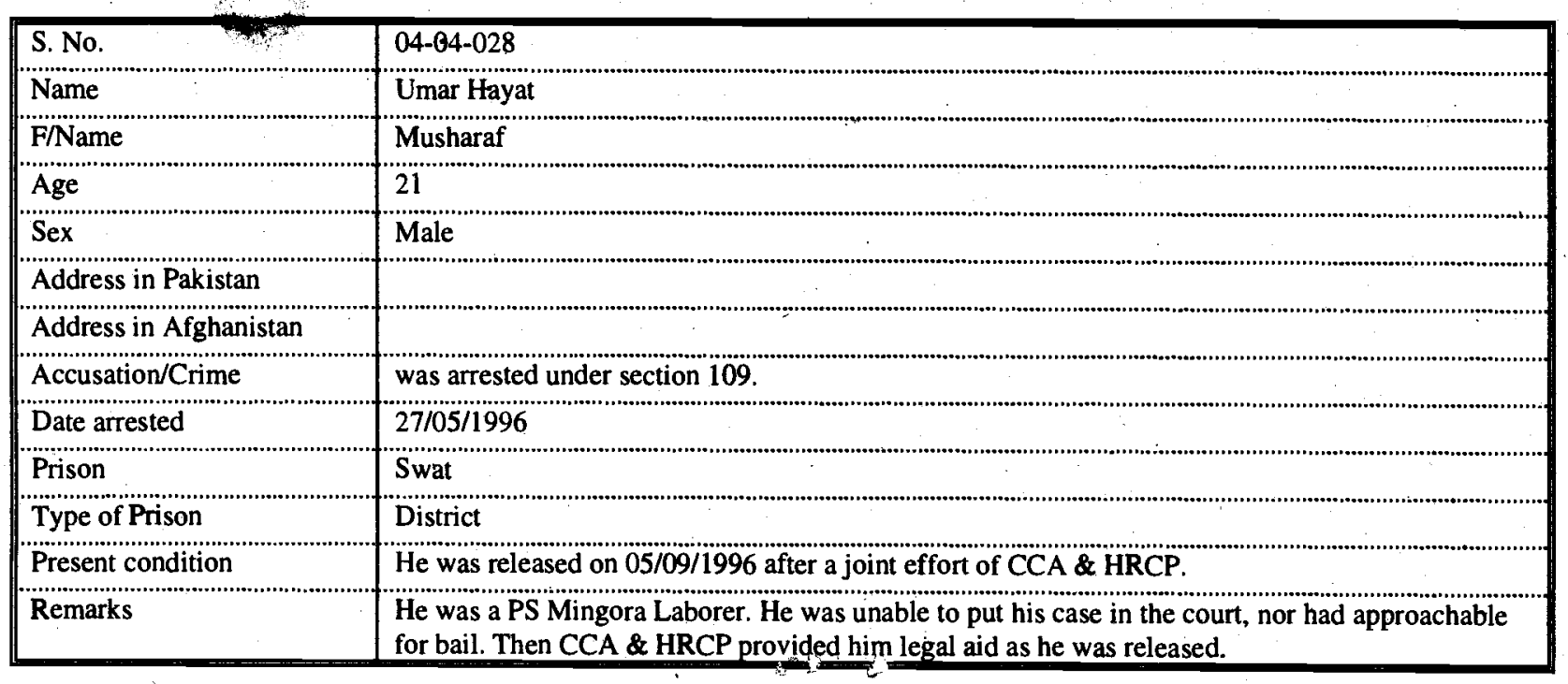




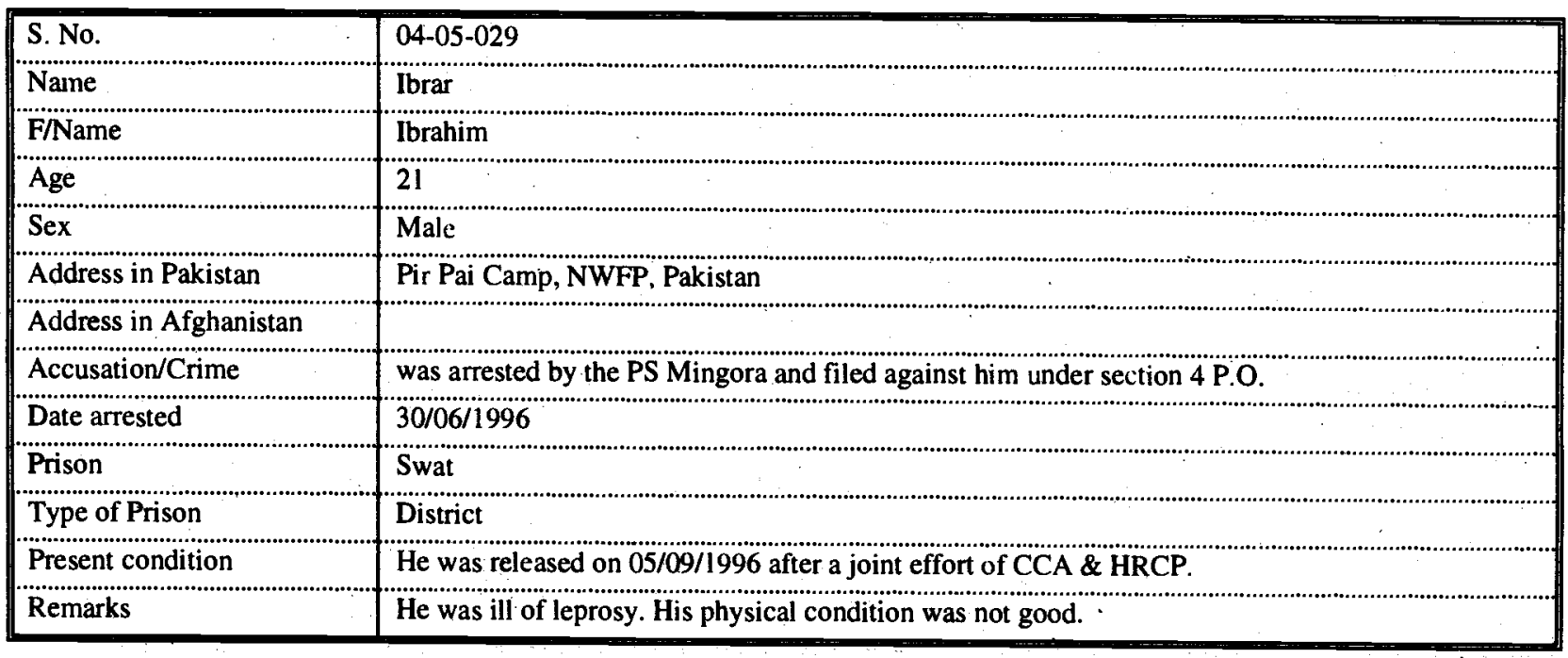

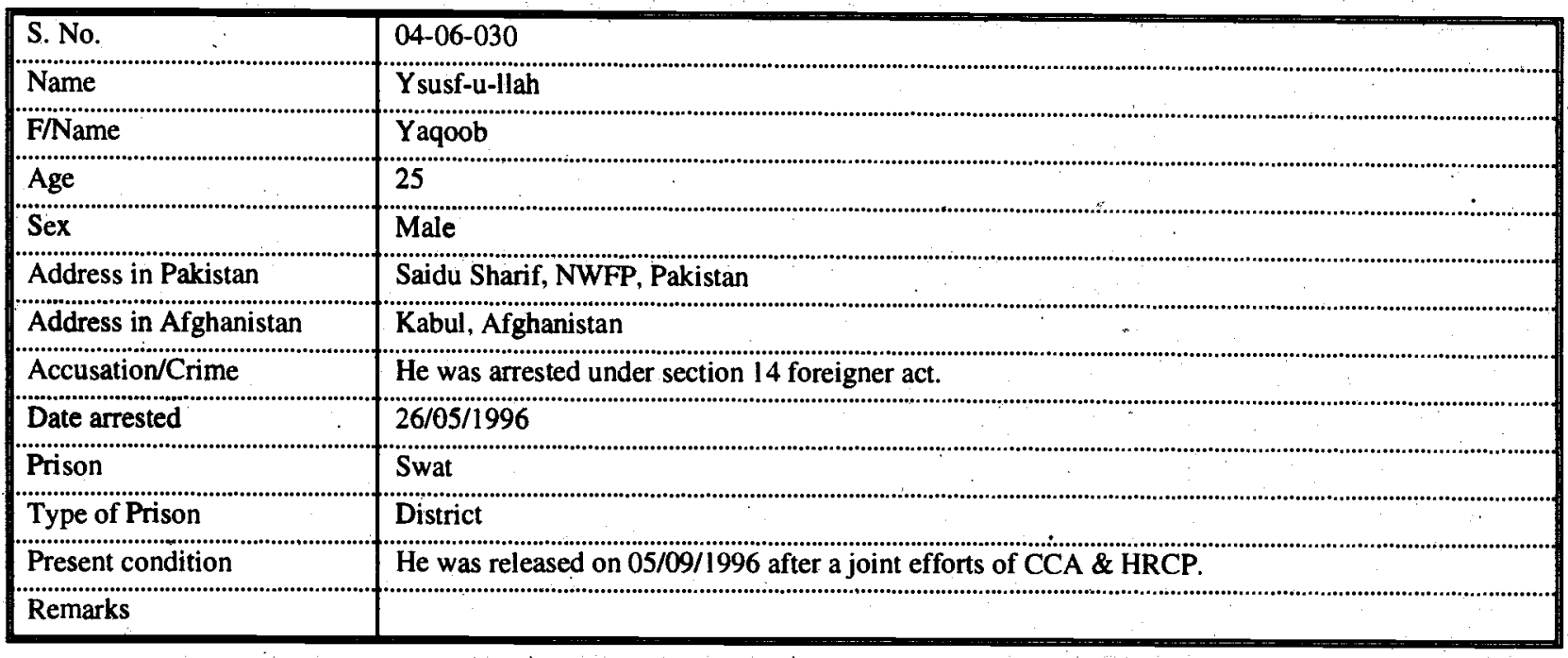

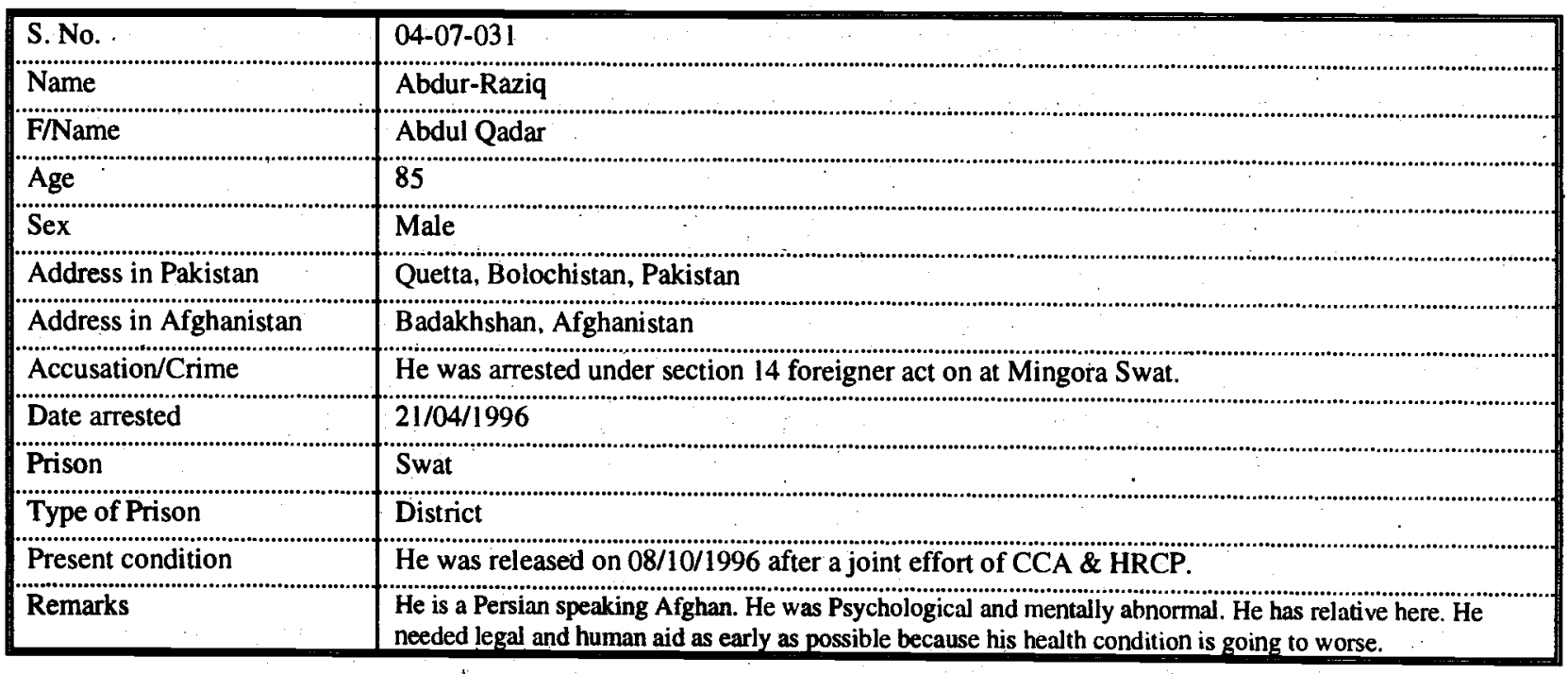




\begin{tabular}{|c|c|}
\hline S. No. & $04-08-032$ \\
\hline Name & Abdul Waheed \\
\hline F/Name & Abdul Manan \\
\hline Age & 35 \\
\hline Sex & Male \\
\hline Address in Pakistan & Gogani Balist, Swat, NWFP, Pakistan \\
\hline \multicolumn{2}{|c|}{ Address in Afghanistan } \\
\hline Accusation/Crime & He was arrested under section 109. \\
\hline \multicolumn{2}{|l|}{ Date arrested } \\
\hline Prison & Swat \\
\hline Type of Prison & District \\
\hline Present condition & He was released on $25 / 08 / 1996$ after a joint effort of CCA \& HRCP. \\
\hline Remarks & \\
\hline
\end{tabular}

\begin{tabular}{|c|c|}
\hline S. No. & $04-09-033$ \\
\hline Name & Frid Gul \\
\hline F/Name & Omar Gul \\
\hline \multicolumn{2}{|l|}{ Age } \\
\hline Sex & Male \\
\hline Address in Pakistan & Takht Bhai, Swat, NWFP, Pakistan \\
\hline Address in Afghanistan & Jalalabad, Afghanistan \\
\hline Accusation/Crime & He was arrested under section 109/107/151 \\
\hline \multicolumn{2}{|l|}{ Date arrested } \\
\hline Prison & Swat \\
\hline Type of Prison & District \\
\hline Present condition & He was released on 16/08/1996 after a joint effort of CCA \& HRCP. \\
\hline Remarks & \\
\hline
\end{tabular}

\begin{tabular}{|c|c|}
\hline S. No. & $04-10-034$ \\
\hline Name & Awal Jan \\
\hline F/Name & Mira Jan \\
\hline \multicolumn{2}{|l|}{ Age } \\
\hline Sex & Male \\
\hline Address in Pakistan & Bajaur, NWFP, Pakistan \\
\hline Address in Afghanistan & Kunar, Afghanistan \\
\hline Accusation/Crime & He was arrested under section $109 / 107 / 151$ \\
\hline \multicolumn{2}{|l|}{ Date arrested } \\
\hline Prison & Swat $\cdots$ \\
\hline Type of Prison & District \\
\hline Present condition & He was released on $16 / 08 / 1996$ after a joint effort of CCA \& HRCP. \\
\hline Remarks & \\
\hline
\end{tabular}




\begin{tabular}{|c|c|}
\hline S. No. & $04-11-035$ \\
\hline Name & Mohammed Wazerullah Khan \\
\hline F/Name & Amirzada \\
\hline \multicolumn{2}{|l|}{ Age } \\
\hline Sex & Male \\
\hline Address in Pakistan & Mingawara, NWFP, Pakistan \\
\hline Address in Afghanistan & Jalalabad, Afghanistan \\
\hline Accusation/Crime & He was arrested under section $107 / 151$ \\
\hline \multicolumn{2}{|l|}{ Date arrested } \\
\hline Prison & Swat \\
\hline Type of Prison & District \\
\hline Present condition & He was released on 15/11/1996 after a joint effort of CCA \& HRCP. \\
\hline Remarks & \\
\hline
\end{tabular}

\begin{tabular}{|c|c|}
\hline S. No. & $04-12-036$ \\
\hline Name & Rasul Mohammed \\
\hline F/Name & Gul Mohammed \\
\hline \multicolumn{2}{|l|}{ Age } \\
\hline Sex & Male \\
\hline Address in Pakistan & Talash Camp, NWFP, Pakistan \\
\hline Address in Afghanistan & Kunar, Afghanistan \\
\hline Accusation/Crime & He was arrested under section 489 . \\
\hline \multicolumn{2}{|l|}{ Date arrested } \\
\hline Prison & Swat \\
\hline Type of Prison & District \\
\hline Present condition & He was released on $15 / 11 / 1996$ after a joint effort of CCA \& HRCP. \\
\hline Remarks & \\
\hline
\end{tabular}

\begin{tabular}{|c|c|}
\hline S. No. & $04-13-037$ \\
\hline Name & Abdul Rashid \\
\hline F/Name & Abdul Hamid \\
\hline Age & 21 \\
\hline Sex & Male \\
\hline Address in Pakistan & Bajaur Camp, NWFP, Pakistan \\
\hline Address in Afghanistan & \\
\hline Accusation/Crime & He was arrested under section $151 / 107$ \\
\hline Date arrested & \\
\hline Prison & Swat \\
\hline Type of Prison & District \\
\hline Present condition & His case is under trail. \\
\hline Remarks & \\
\hline
\end{tabular}




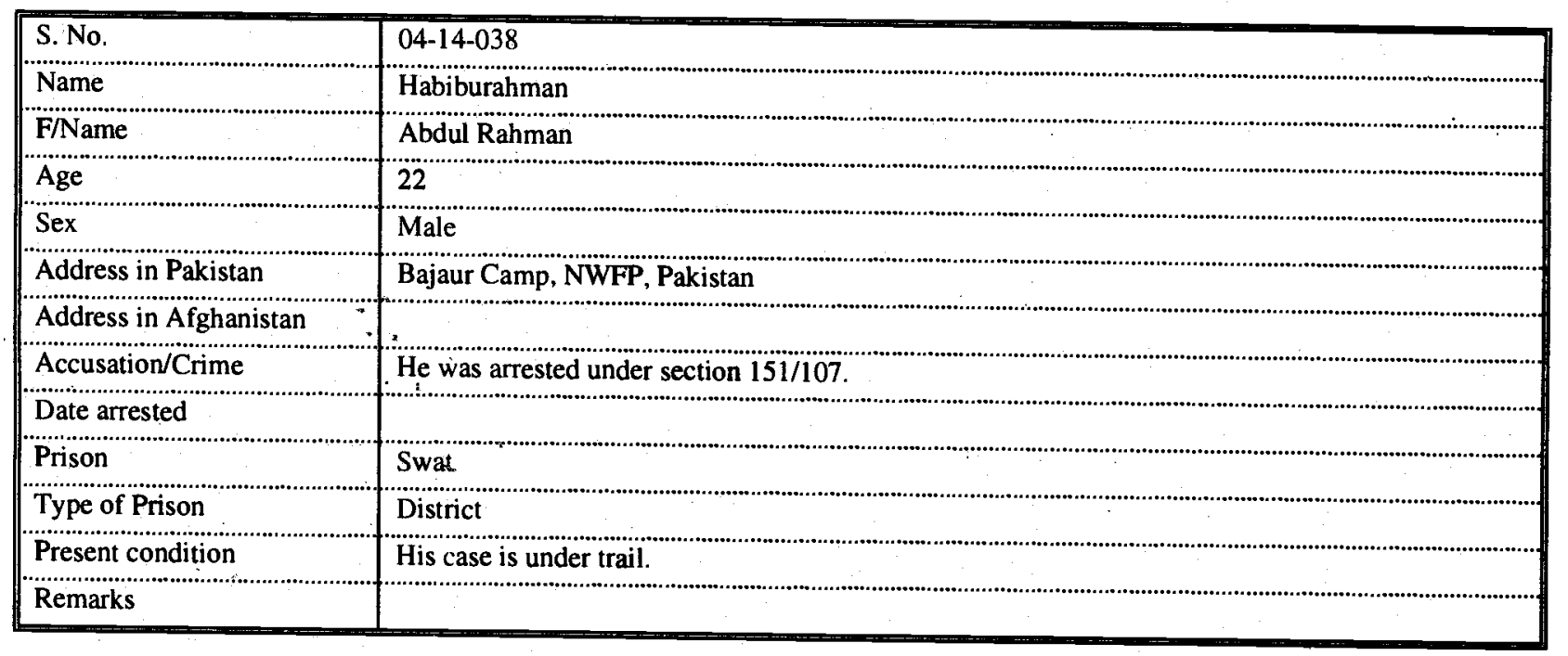

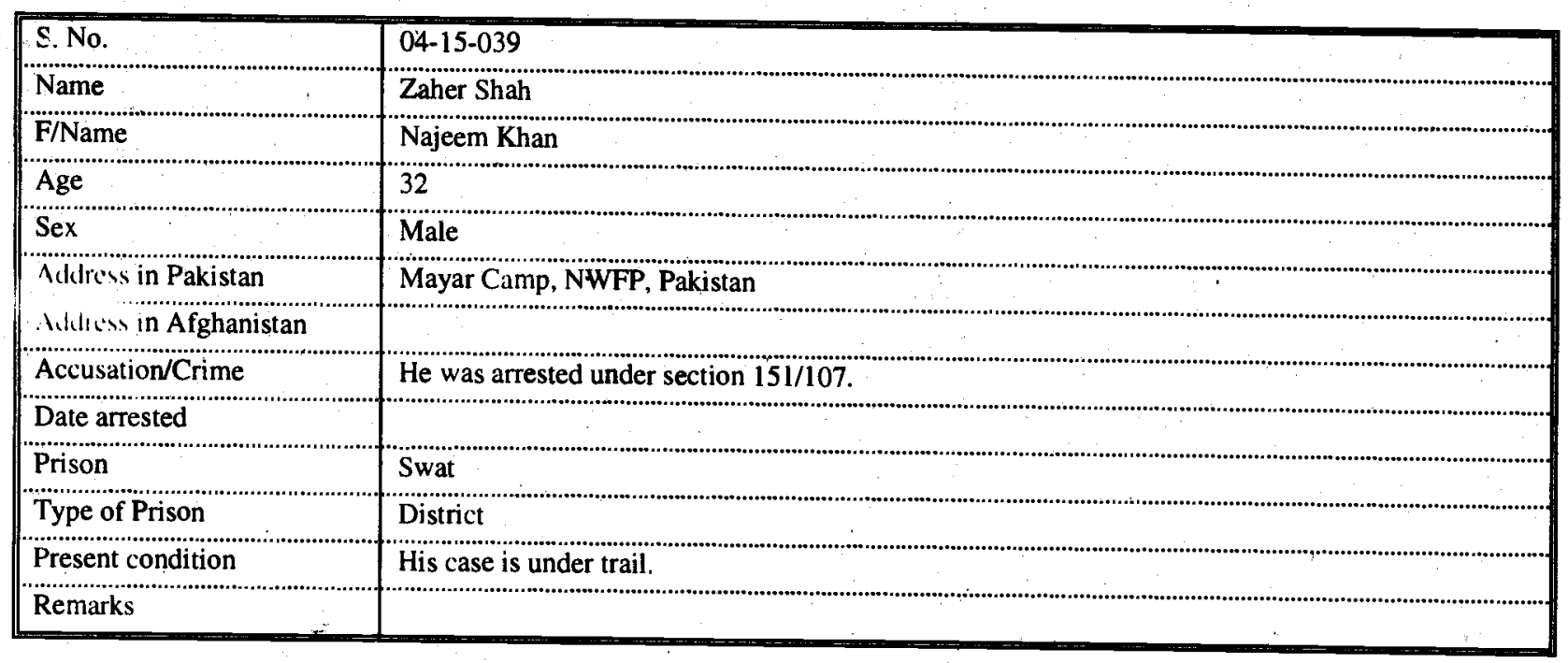




\section{District Prison Charsadda}

\begin{tabular}{|c|c|}
\hline S. No. & 05-01-040 \\
\hline Name & Sher Agha \\
\hline F/Name & Mohammed Tahir \\
\hline Age & 21 \\
\hline Sex & Male \\
\hline Address in Pakistan & Pendi, Pakistan \\
\hline Address in Afghanistan & Jalalabad, Afghanistan \\
\hline Accusation/Crime & He was arrested under section of $Z .0 .5 / 10 / 11$. \\
\hline Date arrested & $07 / 01 / 1996$ \\
\hline Prison & Charsada District \\
\hline Type of Prison & District \\
\hline Present condition & He was released on $22 / 08 / 1996$ after a joint effort of CCA \& HRCP. \\
\hline Remarks & He was arrested by Charsadda Police \\
\hline
\end{tabular}




\begin{tabular}{|c|c|}
\hline S. No. & $05-02-041$ \\
\hline Name & Zahid \\
\hline F/Name & Ghulam \\
\hline \multicolumn{2}{|l|}{ Age } \\
\hline Sex & Male \\
\hline Address in Pakistan & Seri Behlol, Takht Bhai, Pakistan \\
\hline Address in Afghanistan & Jalalabad, Afghanistan \\
\hline Accusation/Crime & He was arrested under section 109. \\
\hline Date arrested & $12 / 01 / 1995$ \\
\hline Prison & Charsada District \\
\hline Type of Prison & District \\
\hline Present condition & He was released on $22 / 08 / 1996$ after a joint effort of CCA \& HRCP. \\
\hline Remarks & \\
\hline
\end{tabular}

\begin{tabular}{|c|c|}
\hline S. No. & $05-03-042$ \\
\hline Name & Yasmin \\
\hline \multicolumn{2}{|l|}{ F/Name } \\
\hline Age & 29 \\
\hline Sex & Female \\
\hline Address in Pakistan & Nisata, Takht Bhai, Pakistan \\
\hline \multicolumn{2}{|c|}{ Address in Afghanistan } \\
\hline Accusation/Crime & She was arrested under section 05/10/11. \\
\hline \multicolumn{2}{|l|}{ Date arrested } \\
\hline Prison & Charsada District \\
\hline Type of Prison & District \\
\hline Present condition & She was released on bail and her case is under trail. \\
\hline Remarks & \\
\hline
\end{tabular}

\begin{tabular}{|c|c|}
\hline S. No. & 05-04-043 \\
\hline Name & Zarkisha \\
\hline \multicolumn{2}{|l|}{ F/Name } \\
\hline \multicolumn{2}{|l|}{ Age } \\
\hline Sex & Female \\
\hline Address in Pakistan & Nisata,Takht Bhai, Pakistan \\
\hline \multicolumn{2}{|c|}{ Address in Afghanistan } \\
\hline Accusation/Crime & She was arrested under section $05 / 10 / 11$. \\
\hline \multicolumn{2}{|l|}{ Date arrested } \\
\hline Prison & Charsada District \\
\hline Type of Prison & District \\
\hline Present condition & She was released on bail and her case is under trail. \\
\hline Remarks & \\
\hline
\end{tabular}




\section{Central Prison Peshawar}

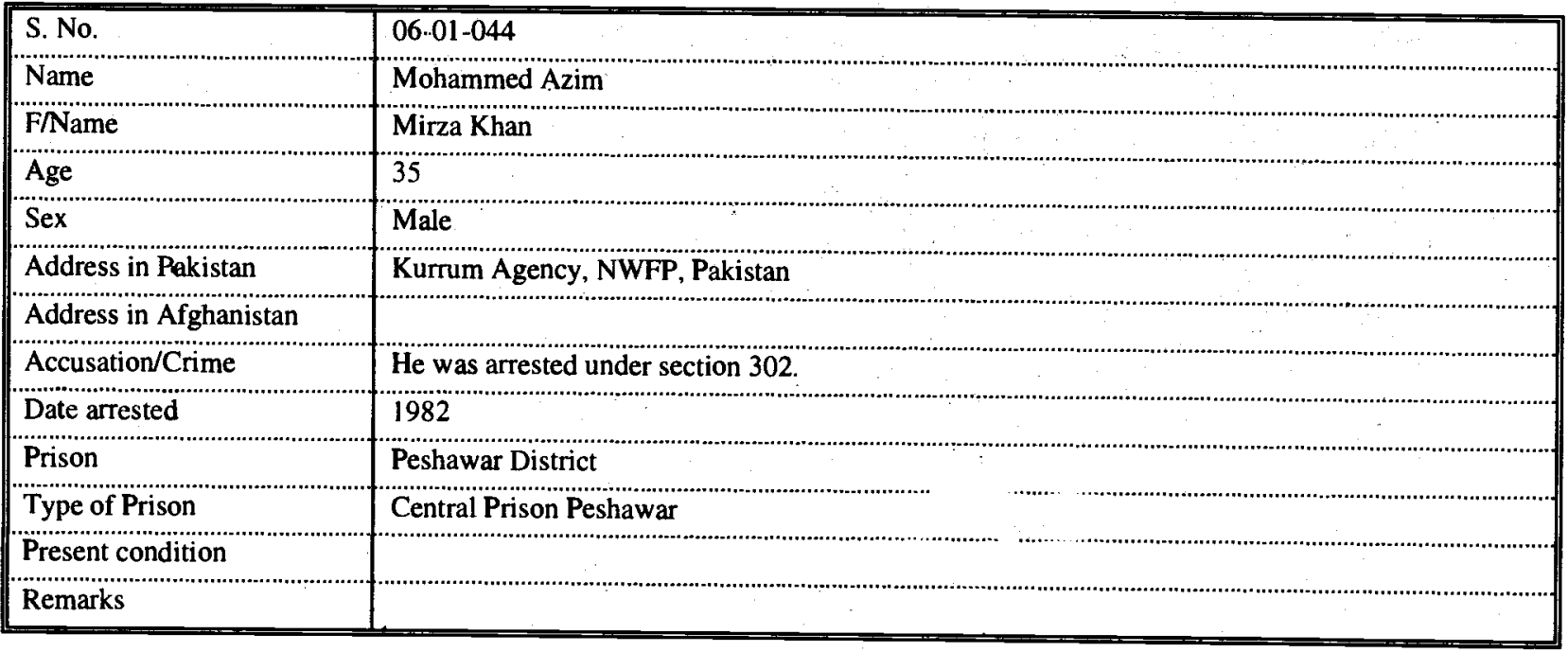




\begin{tabular}{|c|c|}
\hline S. No. & $06-02-045$ \\
\hline Name & Ghulam Rahim \\
\hline F/Name & Khair Mohammed \\
\hline \multicolumn{2}{|l|}{ Age } \\
\hline Sex & Male \\
\hline Address in Pakistan & Nasir Bagh Camp, Peshawar, Pakistan \\
\hline \multicolumn{2}{|c|}{ Address in Afghanistan } \\
\hline \multicolumn{2}{|c|}{ Accusation/Crime } \\
\hline Date arrested & 1960 \\
\hline Prison & Peshawar District \\
\hline Type of Prison & Central Prison Peshawar \\
\hline \multicolumn{2}{|l|}{ Present condition } \\
\hline
\end{tabular}

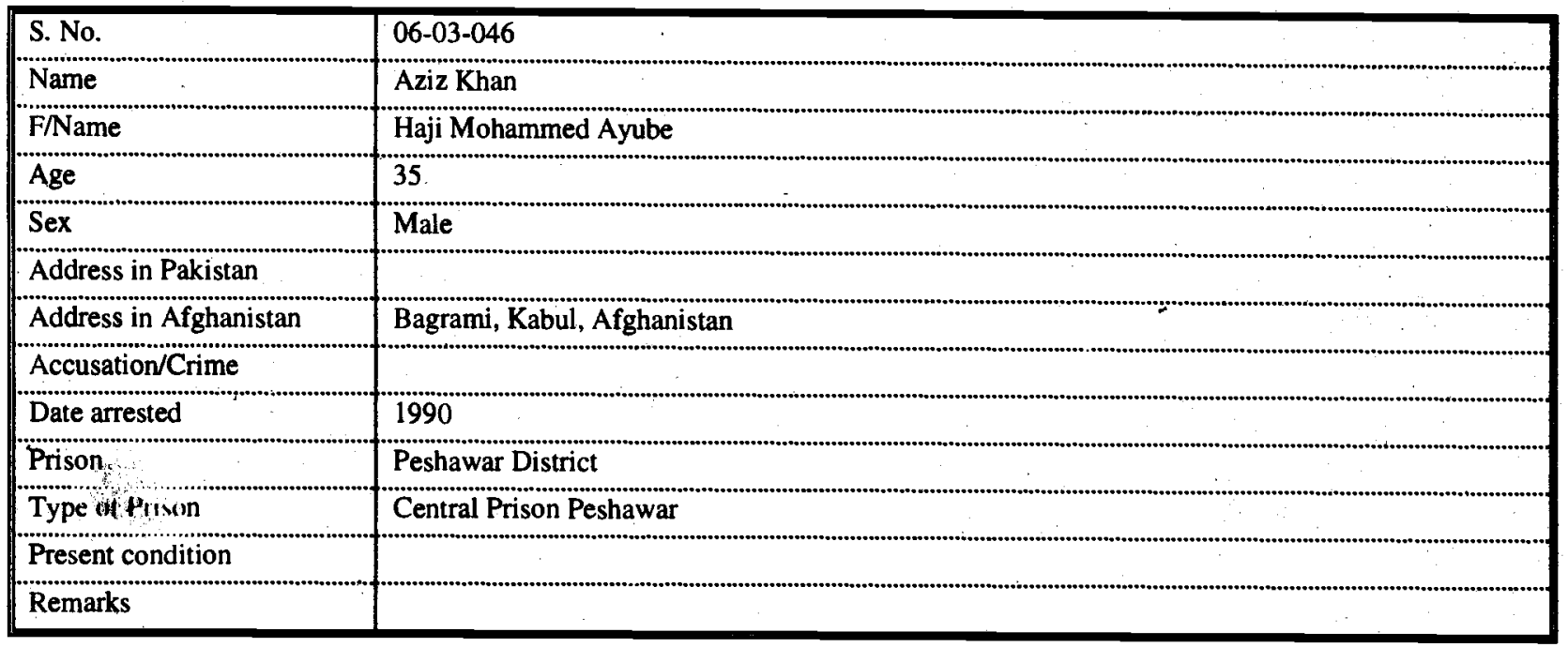

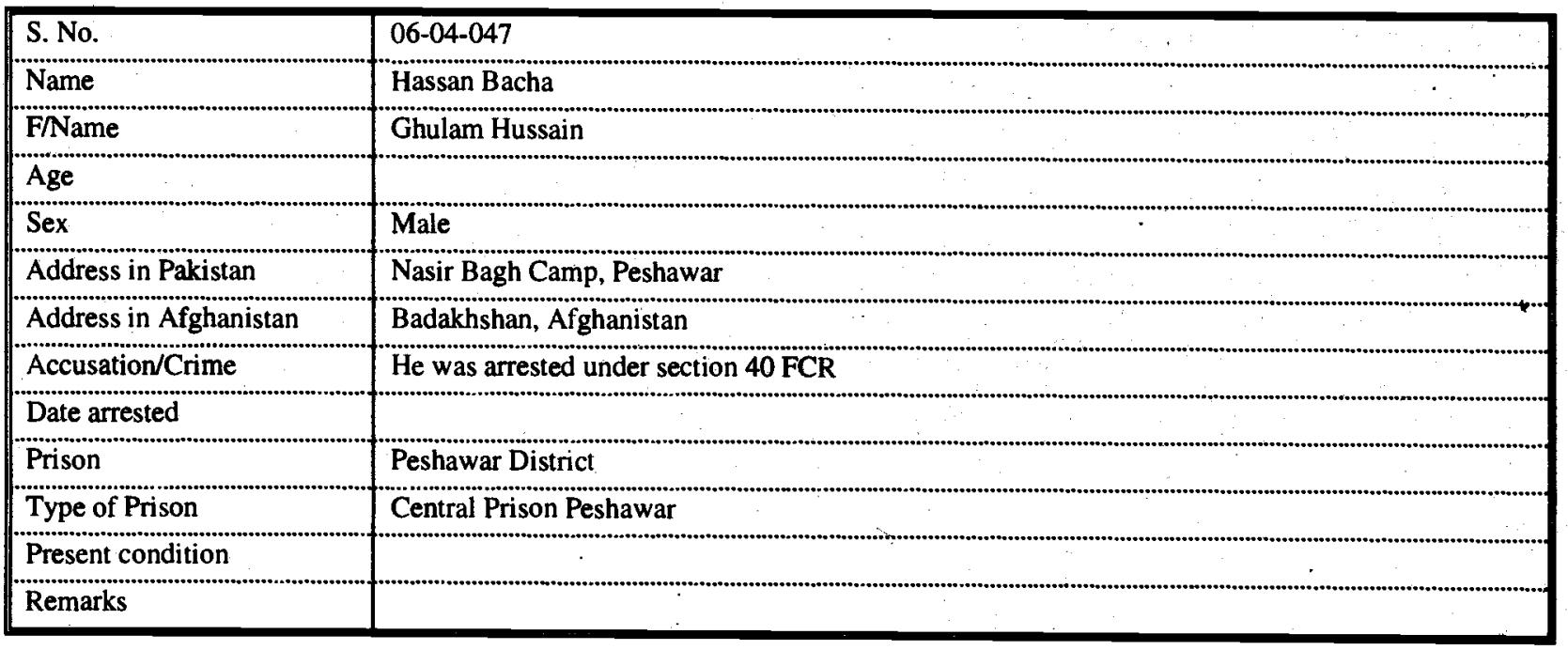




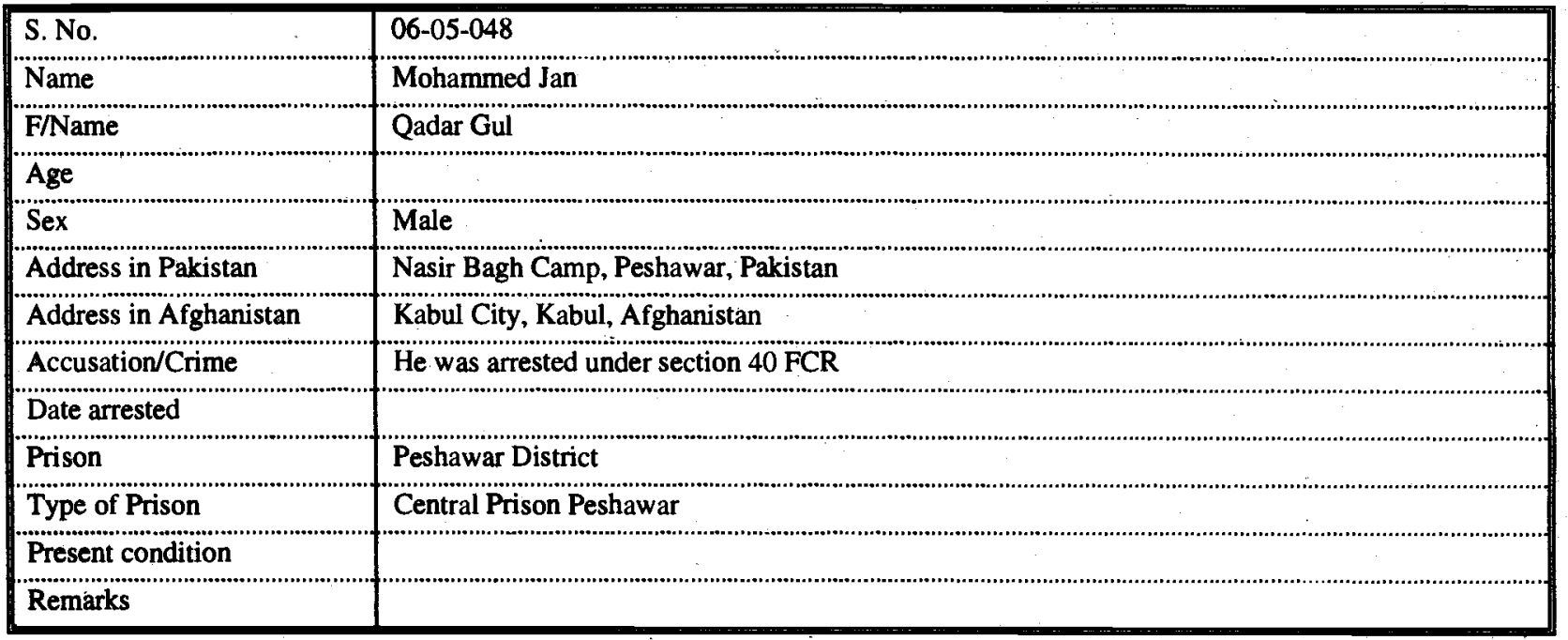

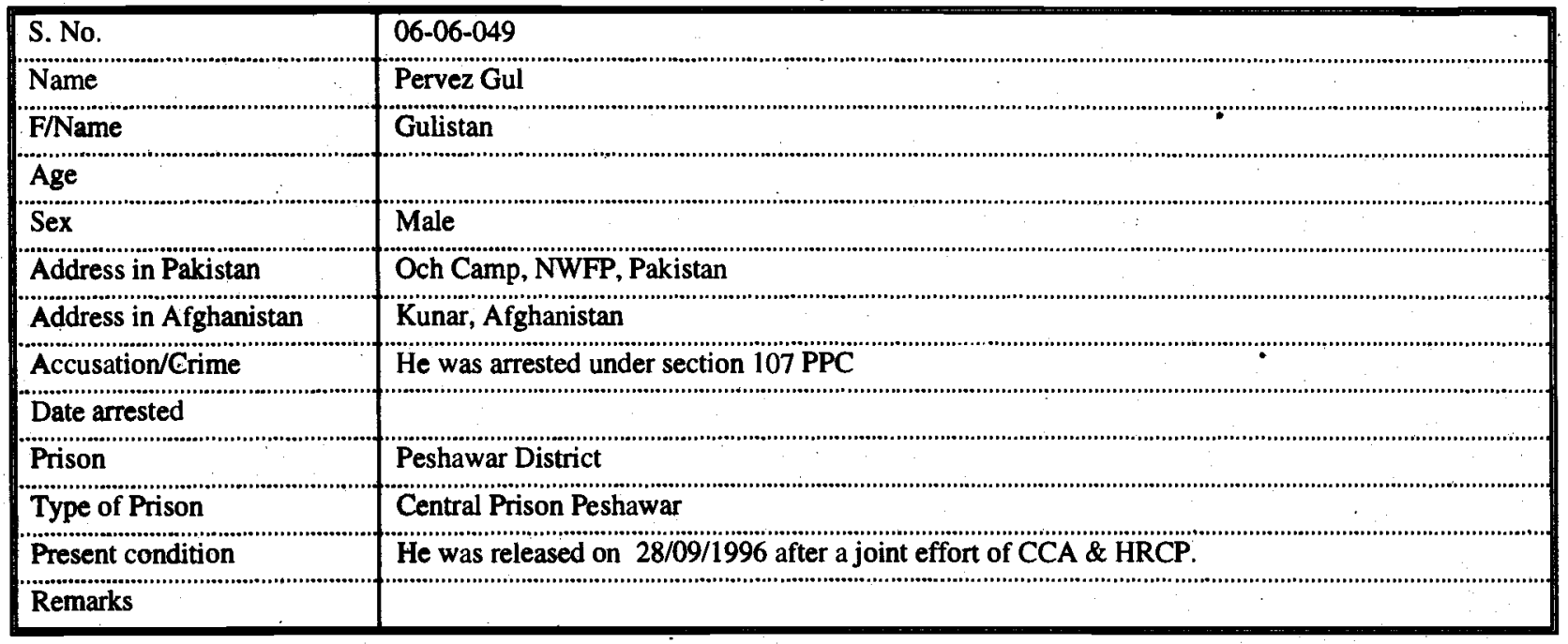

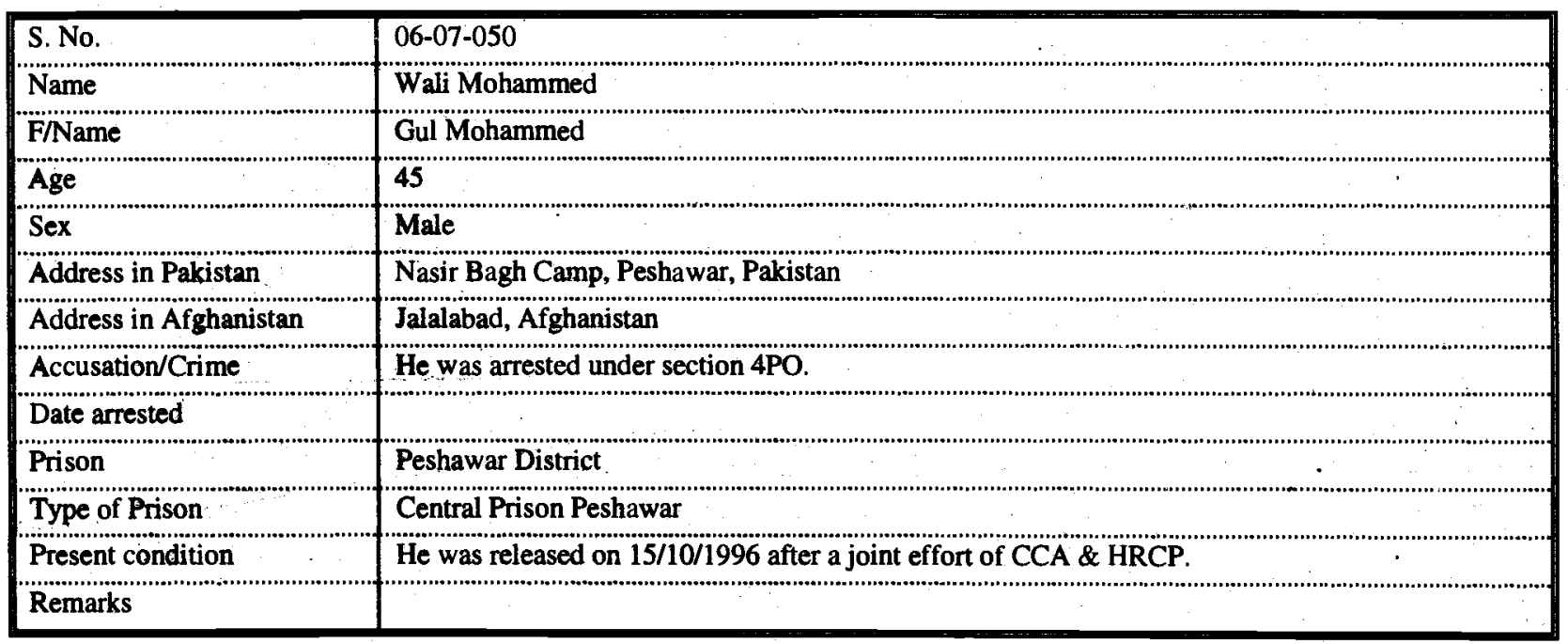




\begin{tabular}{|c|c|}
\hline S. No. & $06-08-051$ \\
\hline F/Name & Ali Akbar \\
\hline Age & 29 \\
\hline Sex & Male \\
\hline Accusation/Crime & He was arrested under section $4 \mathrm{PO}$. \\
\hline \multicolumn{2}{|l|}{ Date arrested } \\
\hline Prison & Peshawar District \\
\hline
\end{tabular}

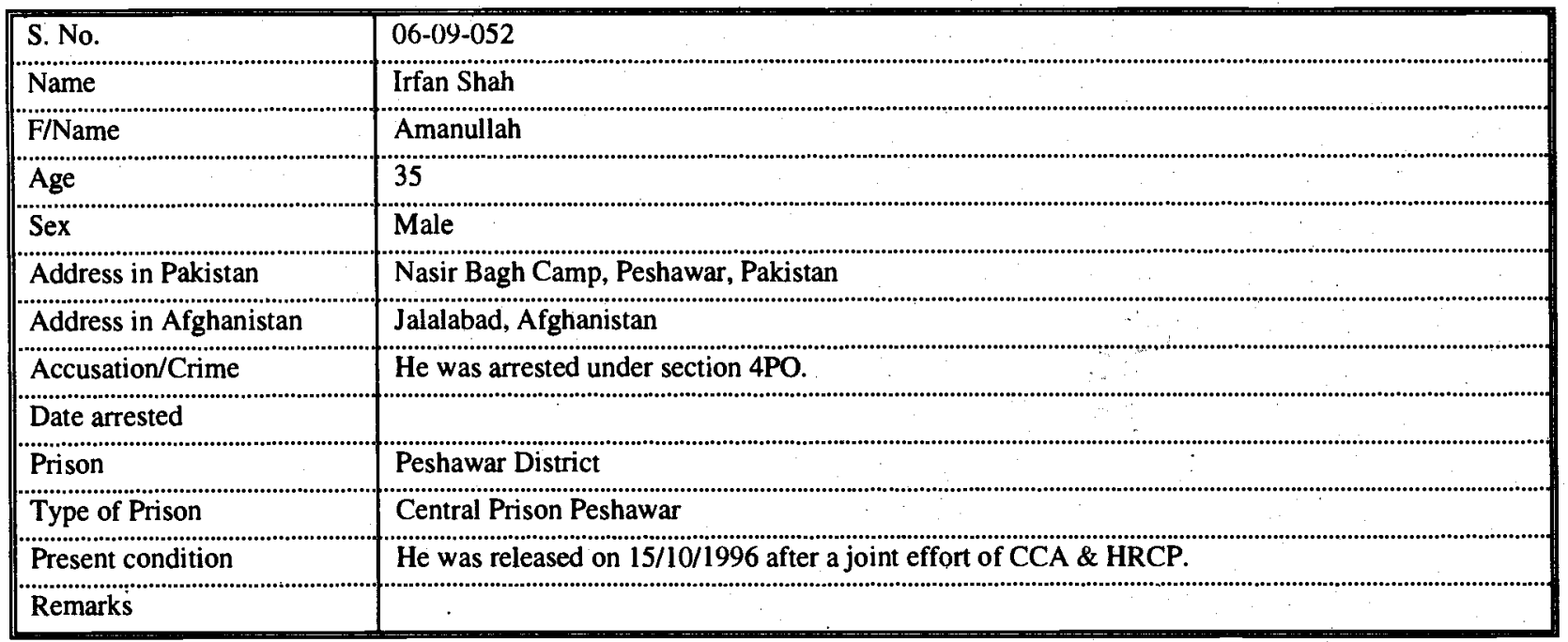

\begin{tabular}{|c|c|}
\hline S. No. & $06-10-053$ \\
\hline Name & Jan Gul \\
\hline F/Name & Abdul Hamid \\
\hline Age & 31 \\
\hline Sex & Male \\
\hline Accusation/Crime & He was arrested under section 4PO \\
\hline Date arrested & ?... \\
\hline Prison & Peshawar District \\
\hline Type of Prison & Central Prison Peshawar \\
\hline
\end{tabular}




\section{:}

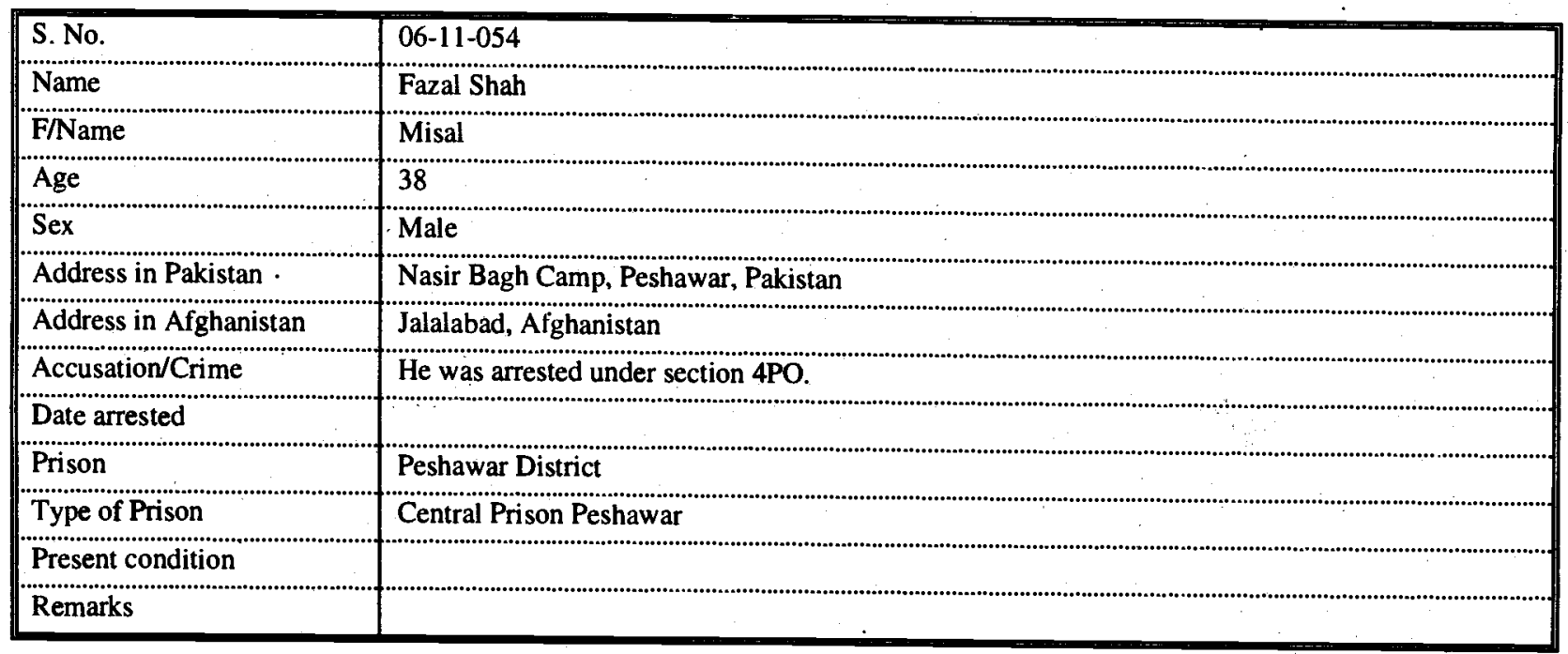

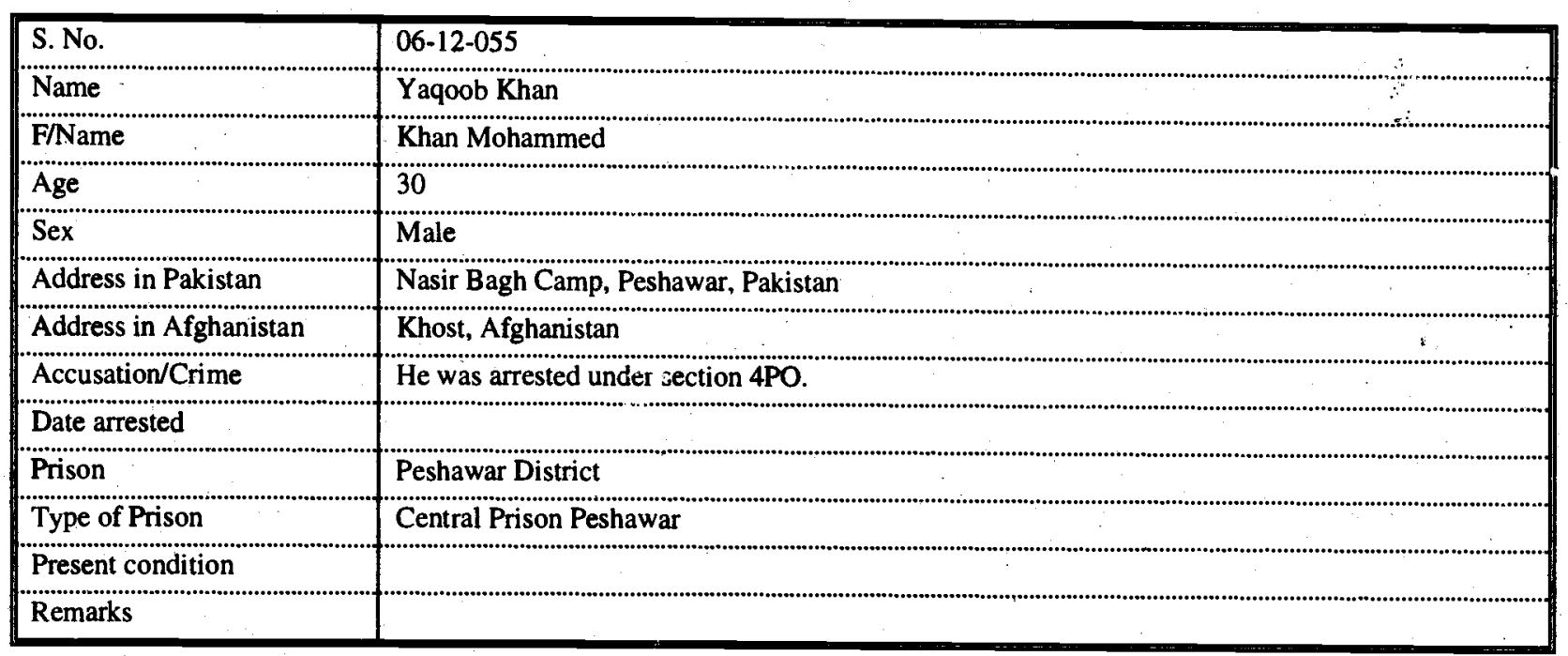

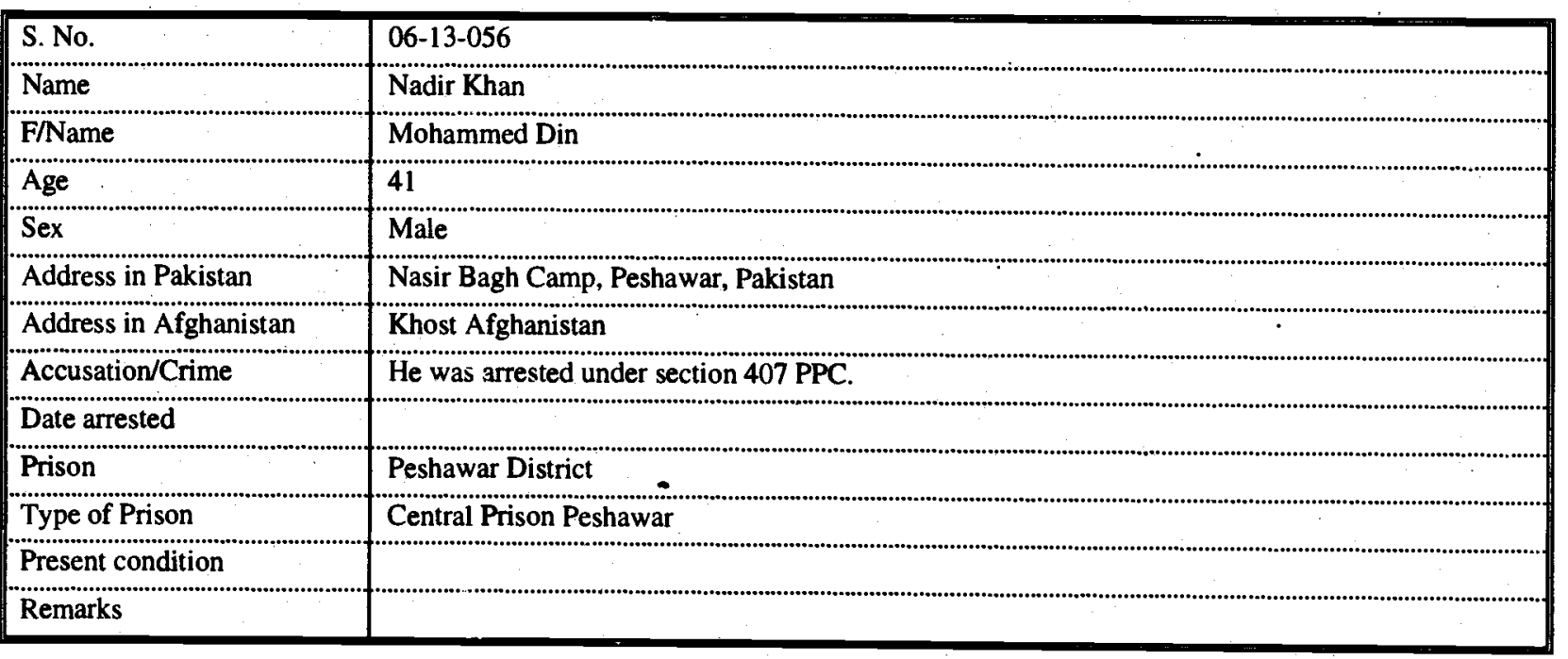




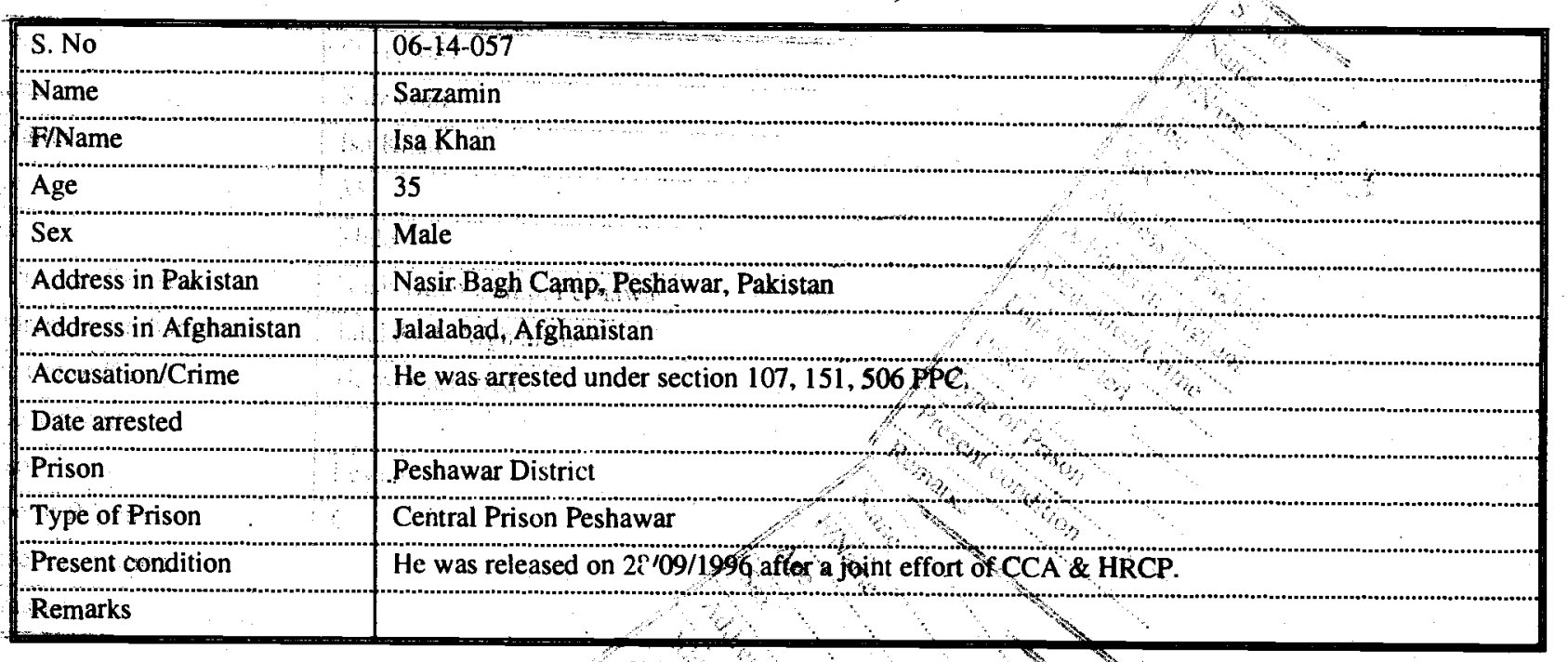

\begin{tabular}{|c|c|}
\hline S. No. & $06-15-058$ \\
\hline Name & Dilawar \\
\hline F/Name & Bakhtrokhan \\
\hline Age & m \\
\hline Sex & Male \\
\hline Address in Pakistan & Nachi Garhi Camp, Peshawar, Pakistan \\
\hline Address in Afghanistan & Jalalabad, Afghanistan \\
\hline Accusation/Crime & He was arreled under section $324,323,107,151$ PPC. \\
\hline Date arrested & $\gamma_{x} \cdot \cdots+\cdots$ \\
\hline Prison & Peshawar District \\
\hline Type of Prison & Central Prison Peshawar \\
\hline Present condition & 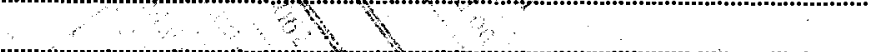 \\
\hline Remarks & \\
\hline S. No. & $06-16-059$ \\
\hline Name & Zardad \\
\hline F/Nâme & Amin \\
\hline Age $\quad 7$ & $28 \times 3$ \\
\hline Sex & Male \\
\hline Address in Pakistan & Nasir Bagh Camp, Peshawar, Pakistan \\
\hline Address in Afghanistan & Jalalabad, Afghanistan \\
\hline Accusation/Crime & He was arrested thinder section $107,109,506$ \\
\hline Date arrested & \\
\hline Prison & Peshawar District \\
\hline Type of Prison & Central Prison Peshawar \\
\hline Present condition & He was released on $28 / 09 / 1996$ after a joint effort of CCA \& HRCP. \\
\hline Remarks & 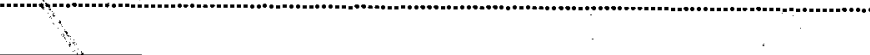 \\
\hline
\end{tabular}




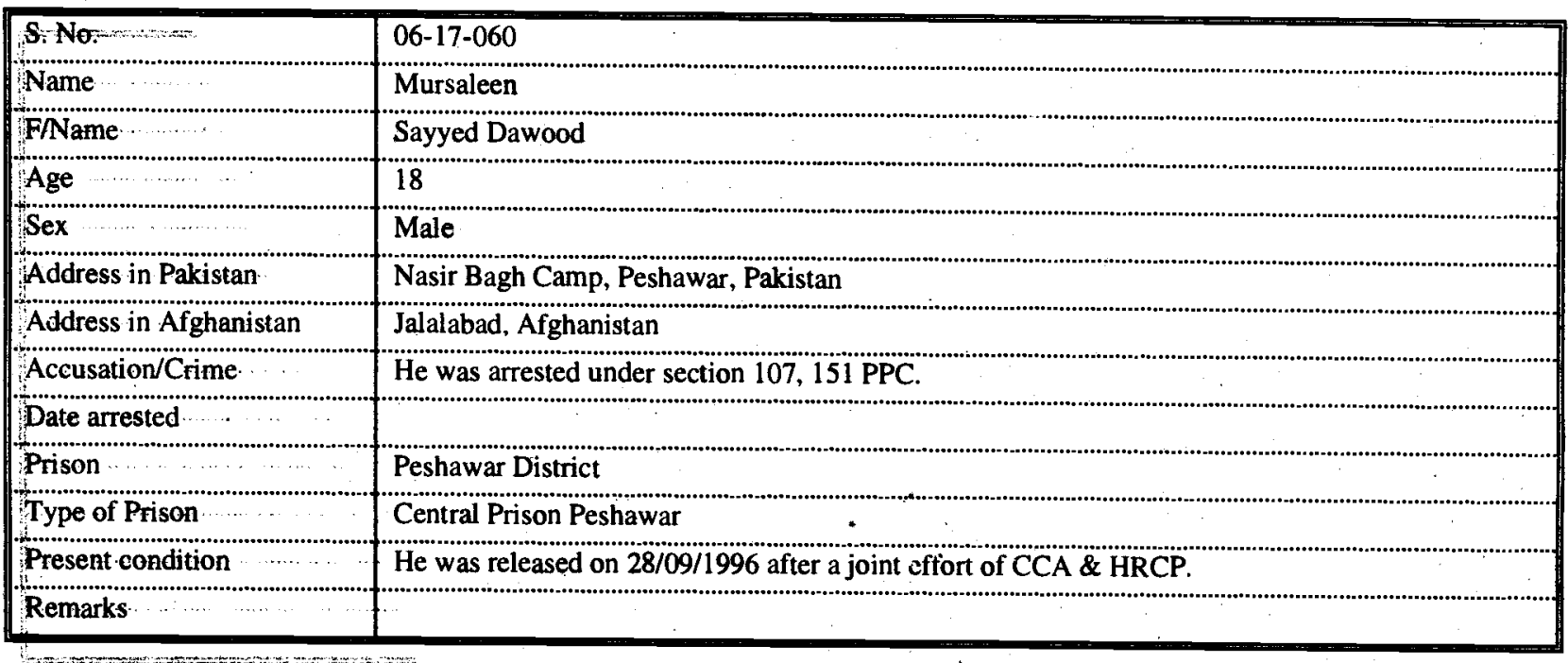

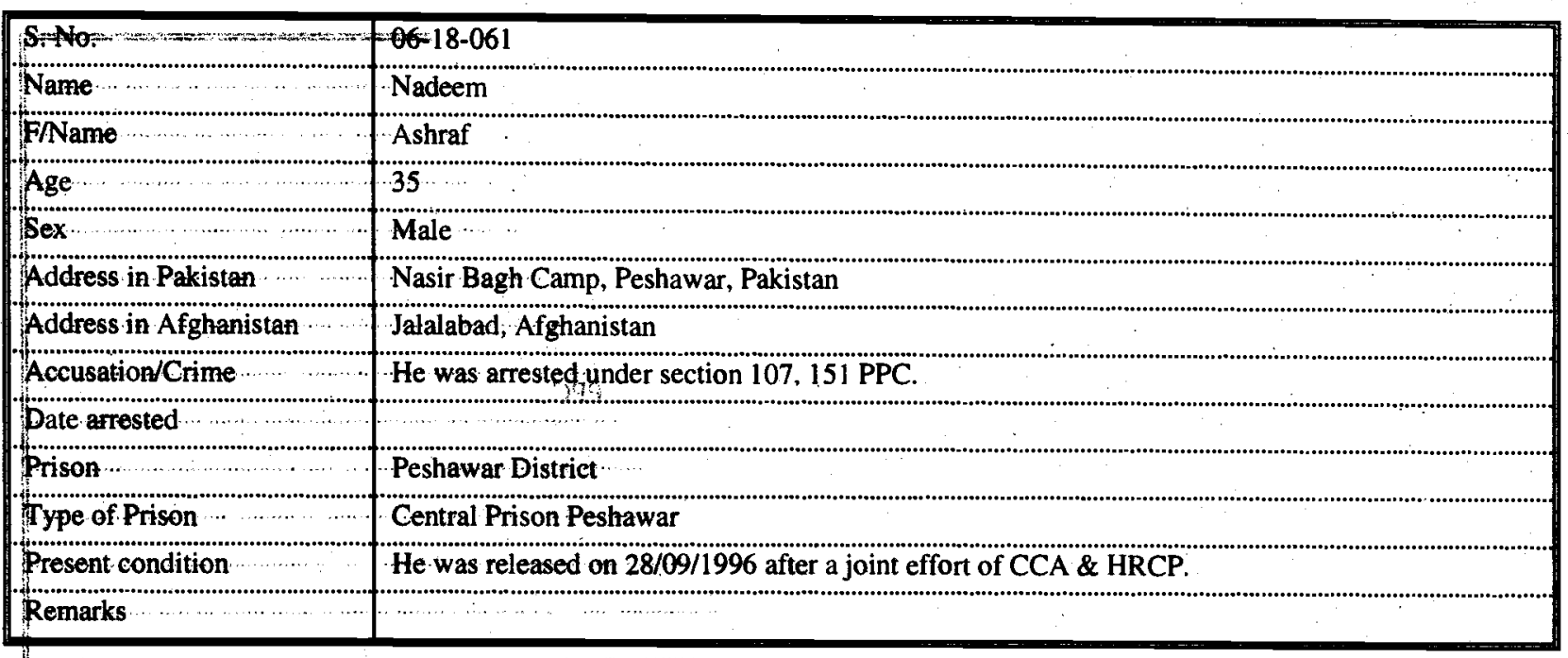

L

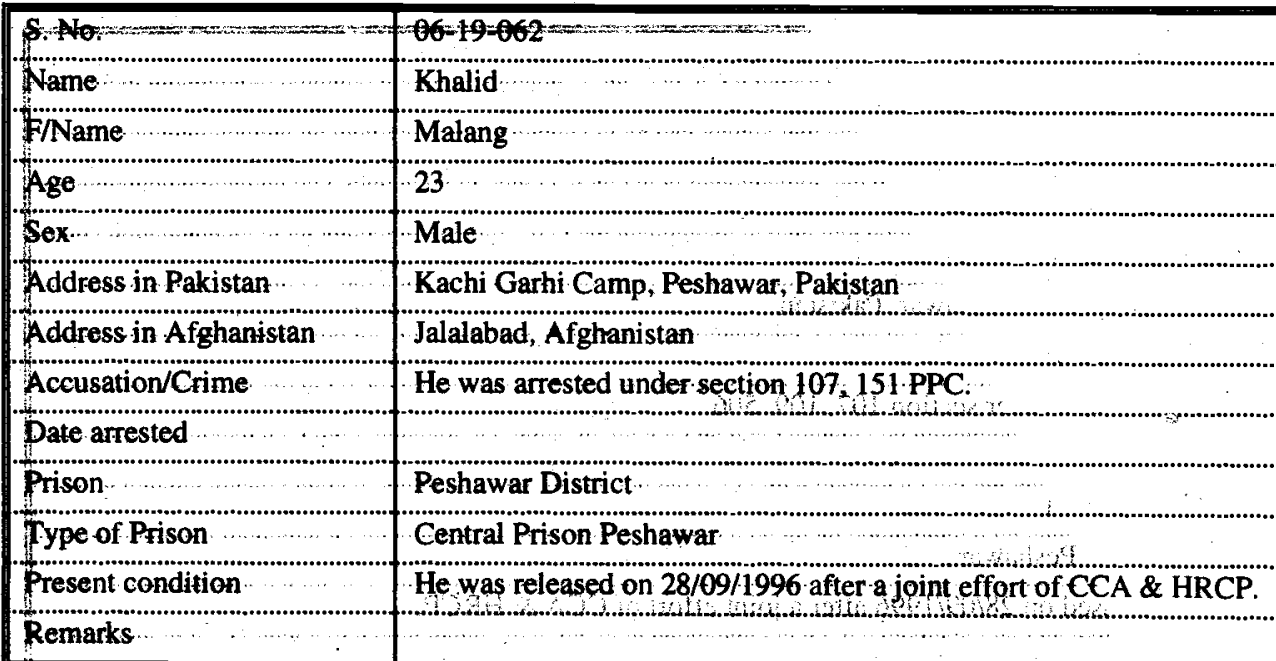

16 of 


\begin{tabular}{|c|c|}
\hline S. No. & $06-20-063$ \\
\hline Name & Faqir \\
\hline F/Name & Malang \\
\hline Age & 23 \\
\hline Sex & Male \\
\hline Address in Pakistan & Kachi Garhi Camp, Peshawar, Pakistan \\
\hline Address in Afghanistan & Jalalabad, Afghanistan \\
\hline Accusation/Crime & He was arrested under section 107,109 PPC. \\
\hline Date arrested & 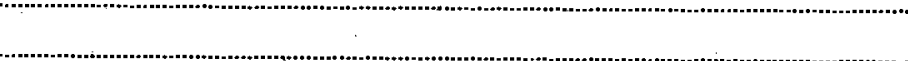 \\
\hline Prison & Peshawar District \\
\hline Type of Prison & Central Prison Peshawar \\
\hline Present condition & He was released on $28 / 09 / 1996$ after a joint effort of CCA \& HRCP. \\
\hline Remarks & $\cdot$ \\
\hline
\end{tabular}




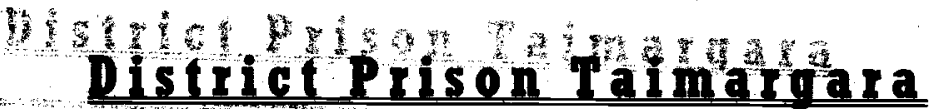

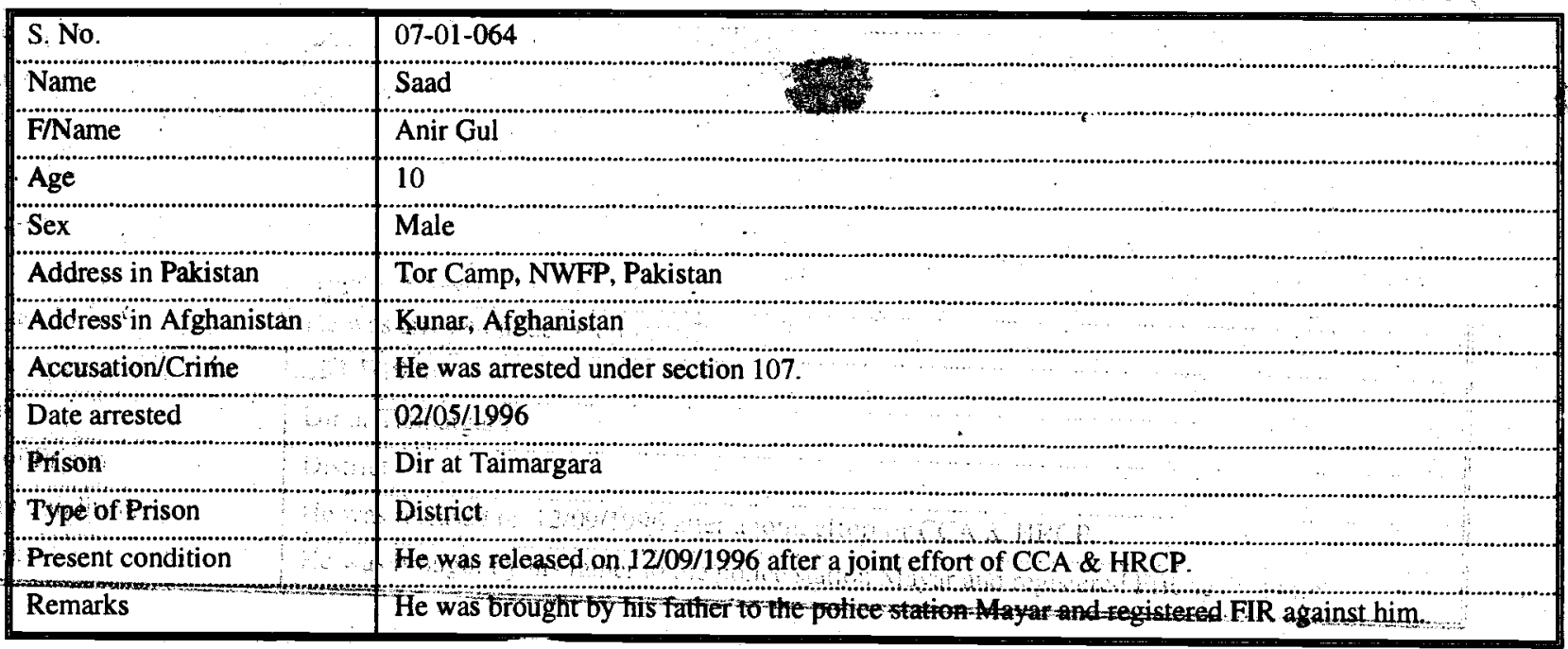




\begin{tabular}{|c|c|}
\hline S. No. & $07-02-065$ \\
\hline Name & Zabihullah \\
\hline F/Name & Mohammed Rahim \\
\hline Age & 9 \\
\hline Sex & Male \\
\hline Address in Pakistan & Taimergara Camp, NWFP, Pakistan \\
\hline Address in Afghanistan & Knuar, Afghanistan \\
\hline Accusation/Crime & He was arrested under 14 Islamic law and 379 . \\
\hline Date arrested & $12 / 02 / 1996$ \\
\hline Prison & Dir at Taimargara \\
\hline Type of Prison & District \\
\hline \multicolumn{2}{|l|}{ Present condition } \\
\hline Remarks & He said to the team that he has no knowledge about the blame. His case not yet pout in the court \\
\hline
\end{tabular}

\begin{tabular}{|c|c|}
\hline S. No. & 07-03-066 \\
\hline Name & Kashar Khan \\
\hline F/Name & Abdul Hassan \\
\hline Age & 20 \\
\hline Sex & Male \\
\hline Address in Pakistan & Barawal Dir, NWFP, Pakistan \\
\hline Address in Afghanistan & Kunar, Afghanistan \\
\hline Accusation/Crime & He was arrested under section 20 5/10/11. \\
\hline \multicolumn{2}{|l|}{ Date arrested } \\
\hline Prison & Dir at Taimargara \\
\hline Type of Prison & District \\
\hline Present condition & He was released on $05 / 10 / 1996$ after a joint effort of CCA \& HRCP. \\
\hline Remarks & \\
\hline
\end{tabular}

\begin{tabular}{|c|c|}
\hline S. No. & 07-04-067 \\
\hline Name & Abdur-Rauf \\
\hline F/Name & Sayyed Amin \\
\hline Age & 18 \\
\hline Sex & Male \\
\hline Address in Pakistan & Barawal Dir, NWFP, Pakistan \\
\hline \multicolumn{2}{|c|}{ Address in Afghanistan } \\
\hline Accusation/Crime & He was arrested under section $\mathrm{ZO} 5 / 10 / 11$. \\
\hline \multicolumn{2}{|l|}{ Date arrested } \\
\hline Prison & Dir at Taimargara \\
\hline Type of Prison & District \\
\hline Present condition & He was released on $05 / 10 / 1996$ atter a joint effort of CCA \& HRCP. \\
\hline Remarks & \\
\hline
\end{tabular}




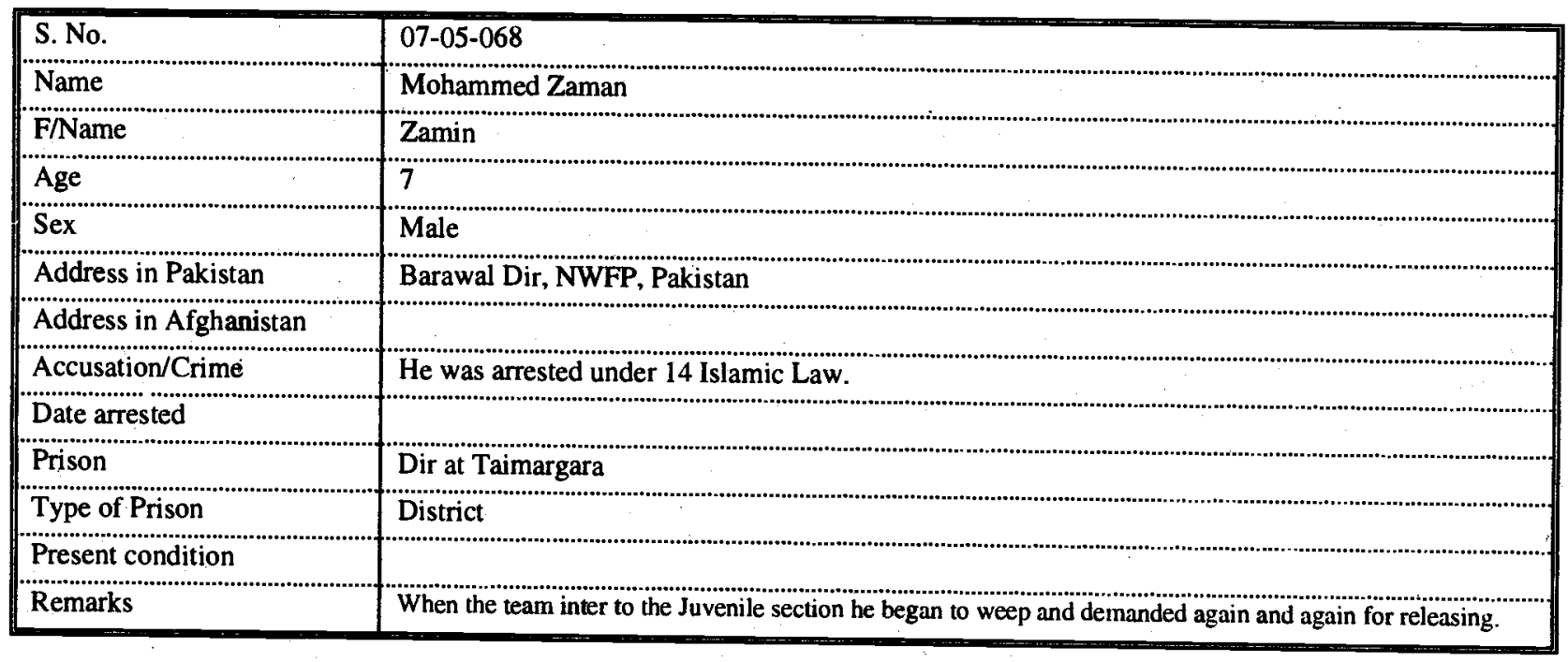

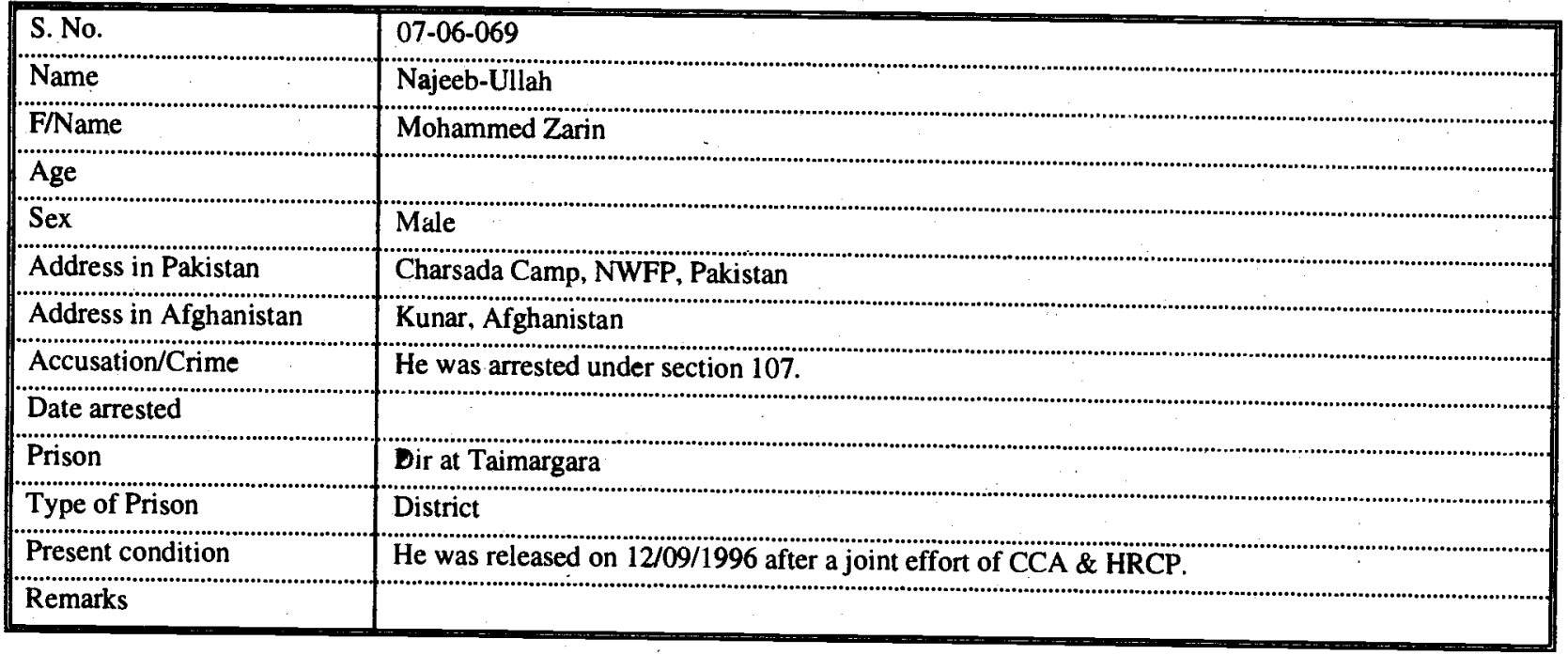

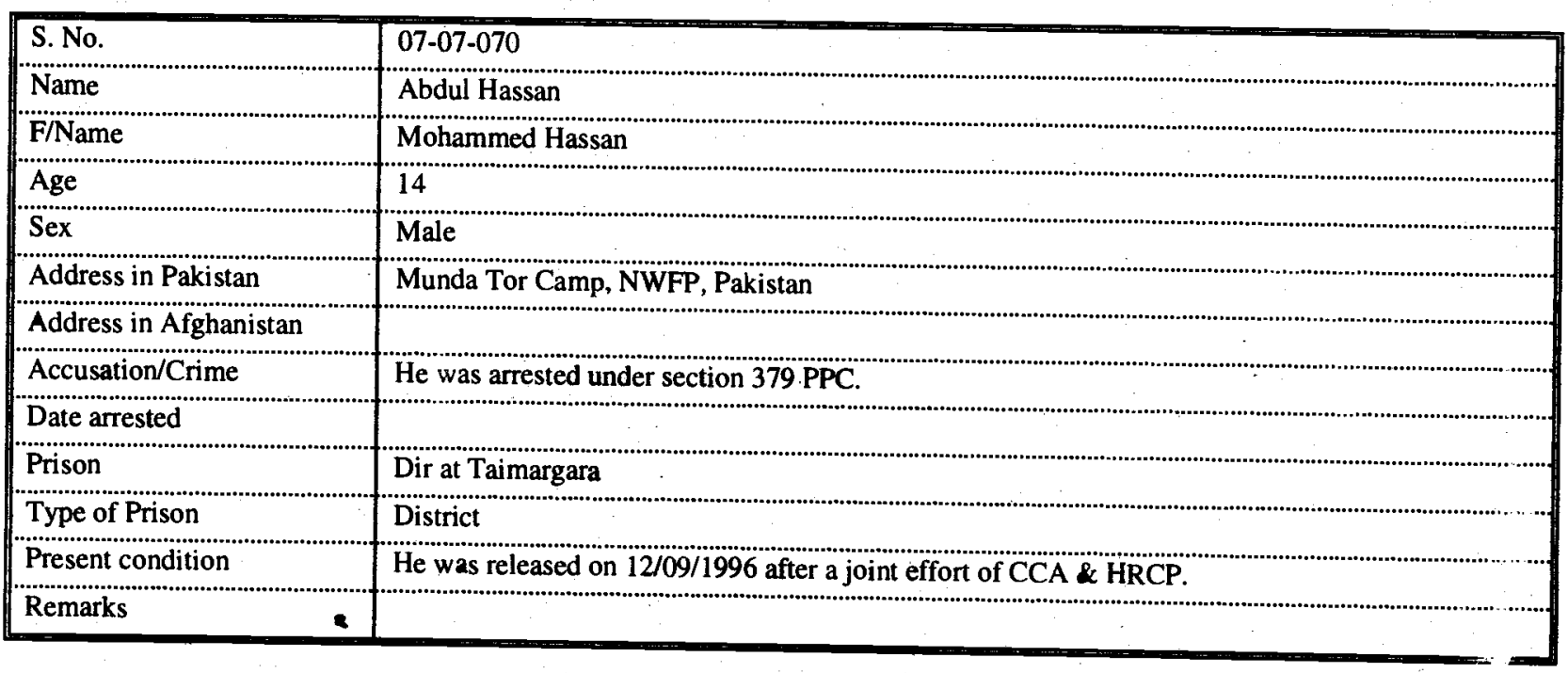




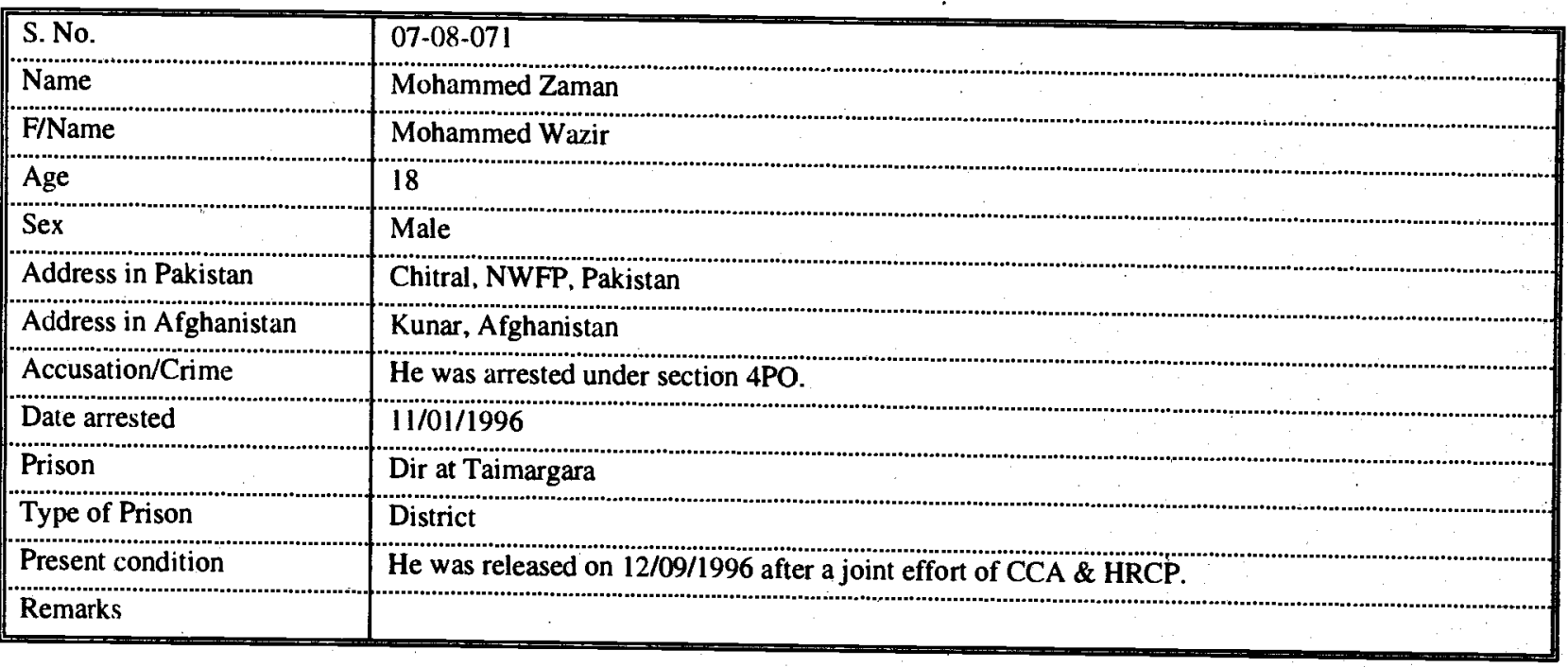

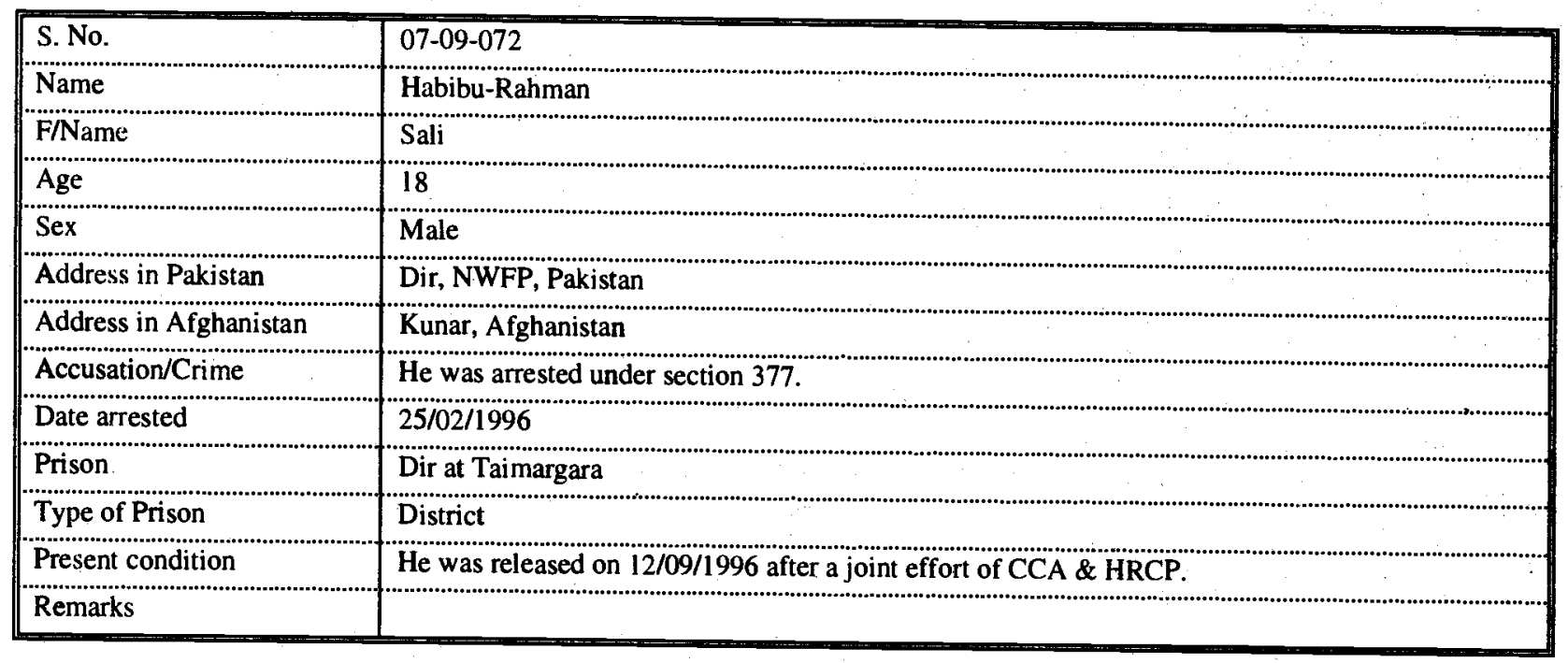

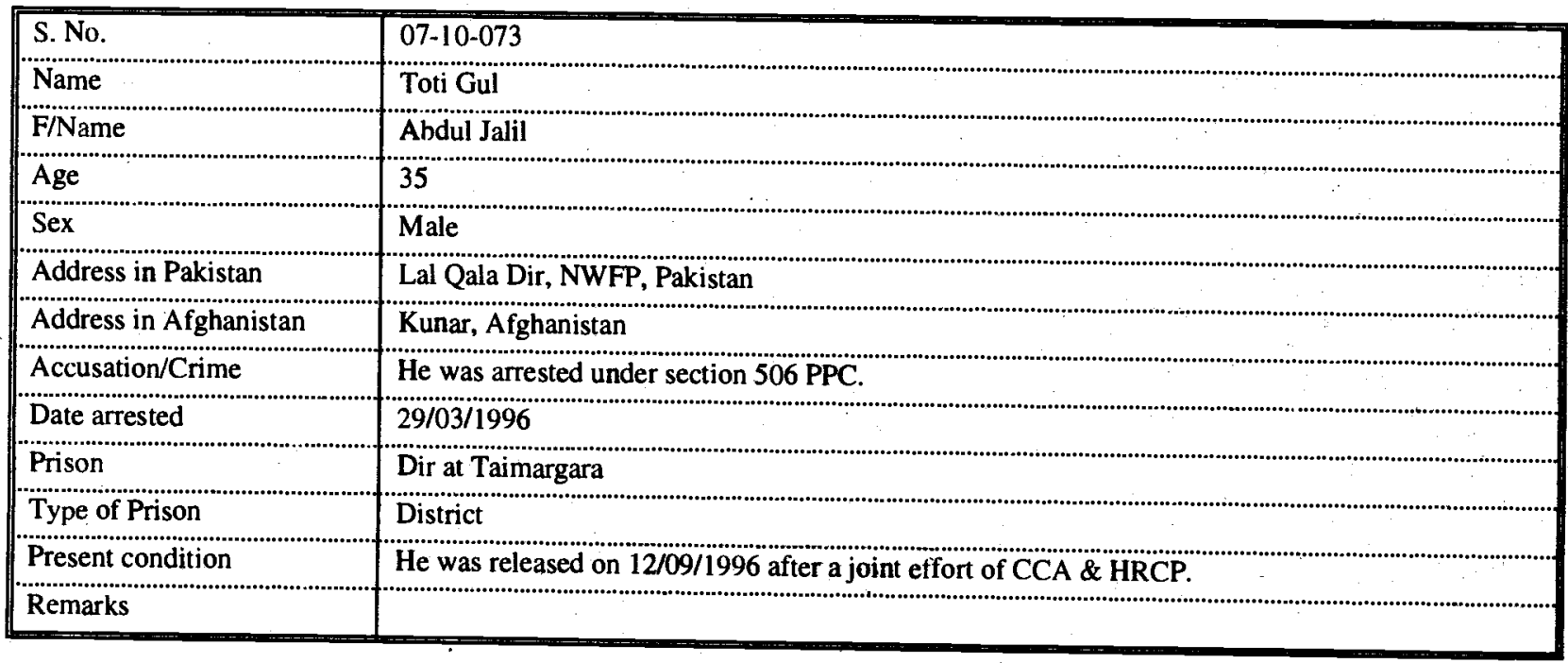




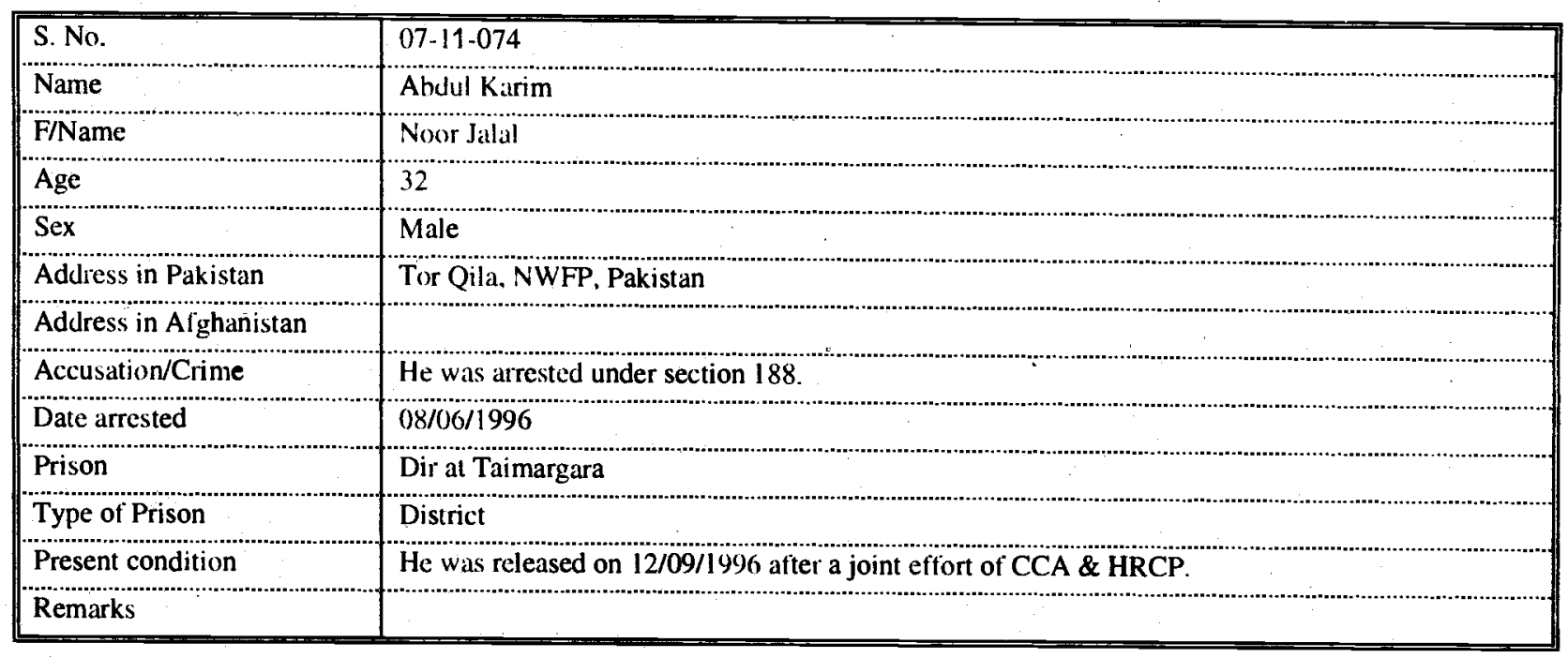

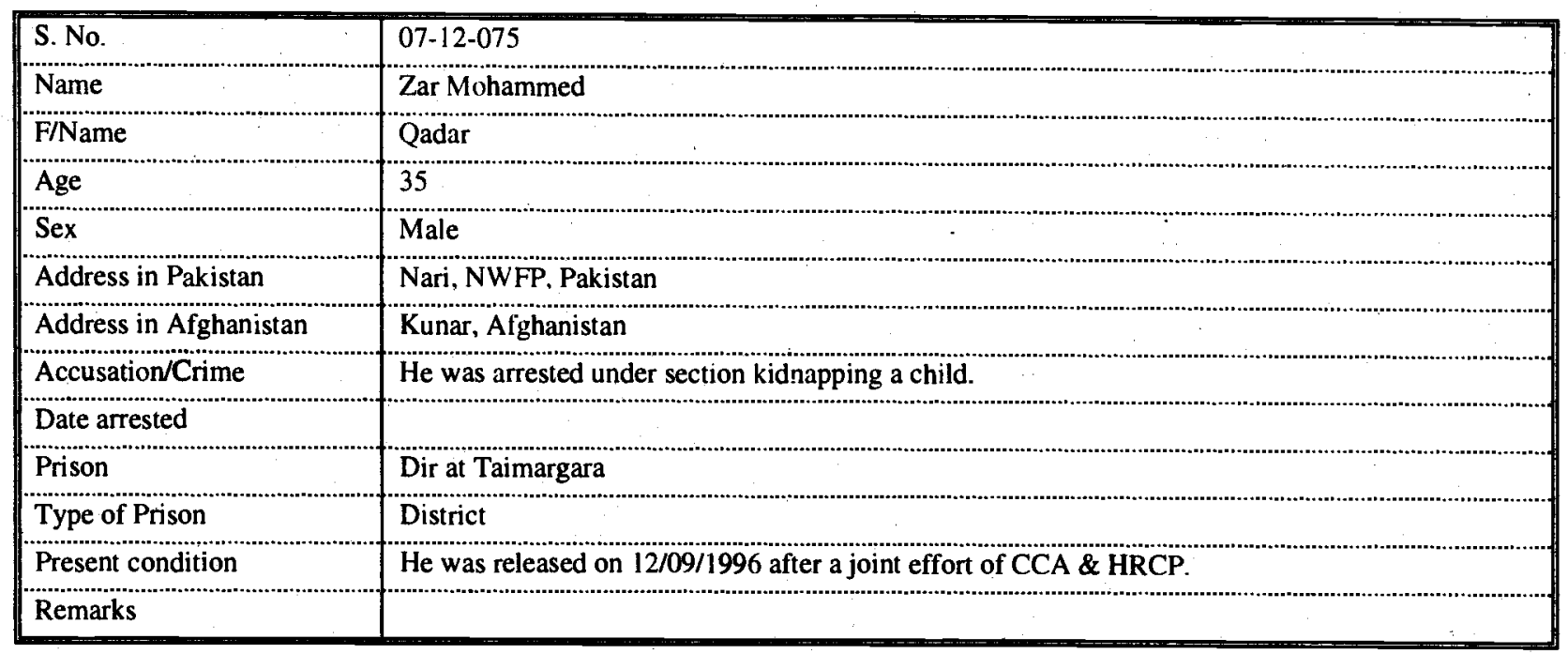

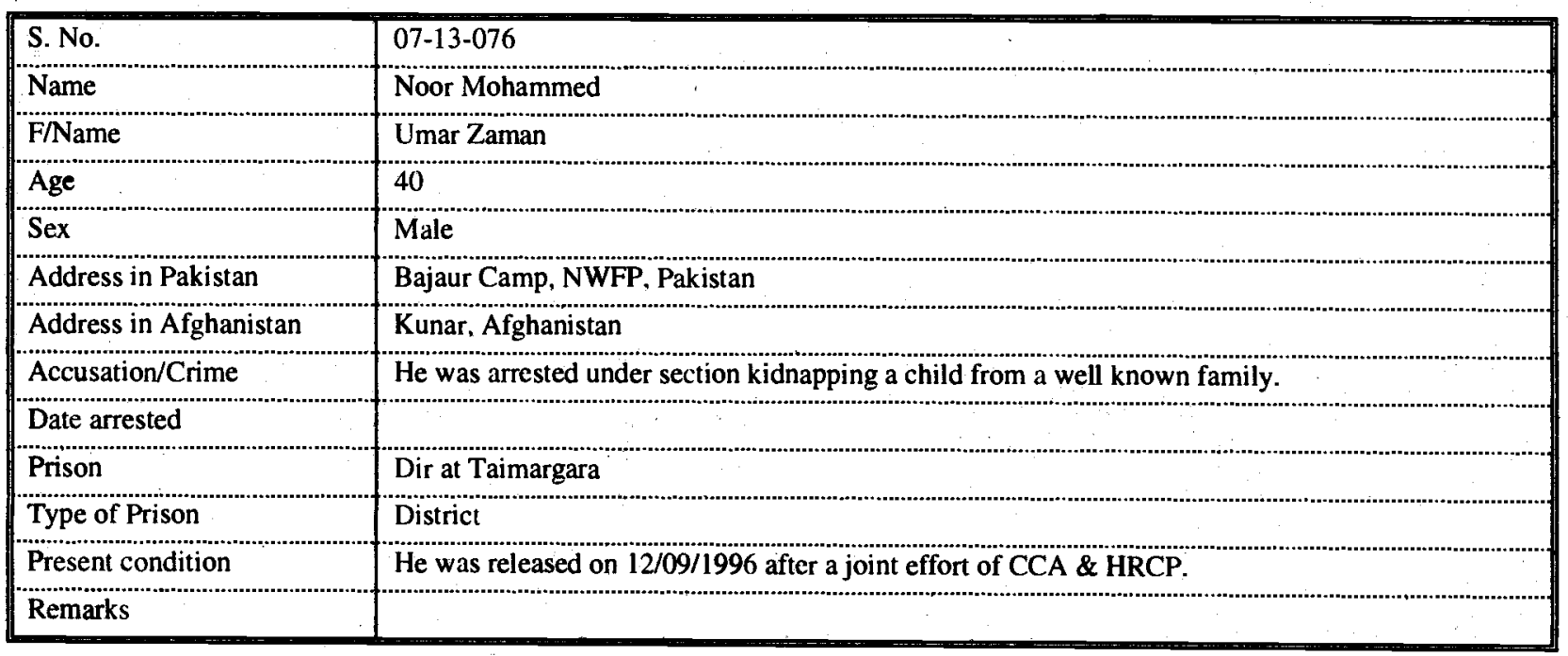




\begin{tabular}{|c|c|}
\hline S. No. & $07-14-077$ \\
\hline Name & Badshah Rehman \\
\hline \multicolumn{2}{|l|}{ F/Name } \\
\hline Age & 25 \\
\hline Sex & Male \\
\hline Address in Pakistan & Tor Camp, NWFP, Pakistan \\
\hline \multicolumn{2}{|l|}{ Address in Afghanistan } \\
\hline Accusation/Crime & He was arrested under section 307 PPC. \\
\hline Date arrested & $02 / 06 / 1996$ \\
\hline Prison & Dir at Taimargara \\
\hline Type of Prison & District \\
\hline Present condition & He was released on 12/09/1996 after a joint effort of CCA \& HRCP. \\
\hline Remarks & \\
\hline
\end{tabular}

\begin{tabular}{|c|c|}
\hline S. No. & 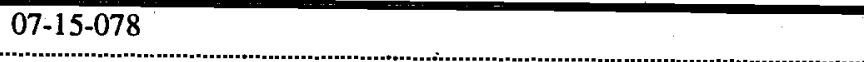 \\
\hline Name & Gul Mohammed \\
\hline F/Name & Wali Mohammed \\
\hline \multicolumn{2}{|l|}{ Age } \\
\hline Sex & Male \\
\hline Address in Pakistan & Och Camp, NWFP, Pakistan \\
\hline Address in Afghanistan & 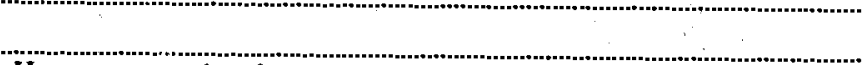 \\
\hline Accusation/Crime & He was arrested under section 107 PPC. \\
\hline Date arrested & $06 / 06 / 1996$ \\
\hline Prison & Dir at Taimargara \\
\hline Type of Prison & District \\
\hline Present condition & He was released on $12 / 09 / 1996$ after a joint effort of CCA \& HRCP. \\
\hline Remarks & 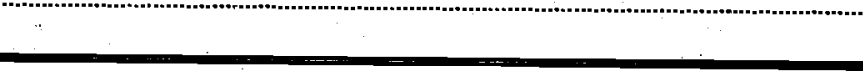 \\
\hline
\end{tabular}

\begin{tabular}{|l|l|l|}
\hline S. No. & $07-16-079$ \\
\hline Name & Fazli Aziz & \\
\hline F/Name & Fazal Rahim & \\
\hline Age & 25 & Male \\
\hline Sex & Samar Bagh, NWFP, Pakistan \\
\hline Address in Pakistan & Kunar, Afghanistan \\
\hline Address in Afghanistan & He was arrested under section 107 PPC \\
\hline Accusation/Crime & $05 / 04 / 1996$ \\
\hline Date arrested & Dir at Taimargara \\
\hline Prison & District \\
\hline Type of Prison & He was released on 12/09/1996 after a joint effort of CCA \& HRCP. \\
\hline Present condition & \\
\hline Remarks & \\
\hline
\end{tabular}




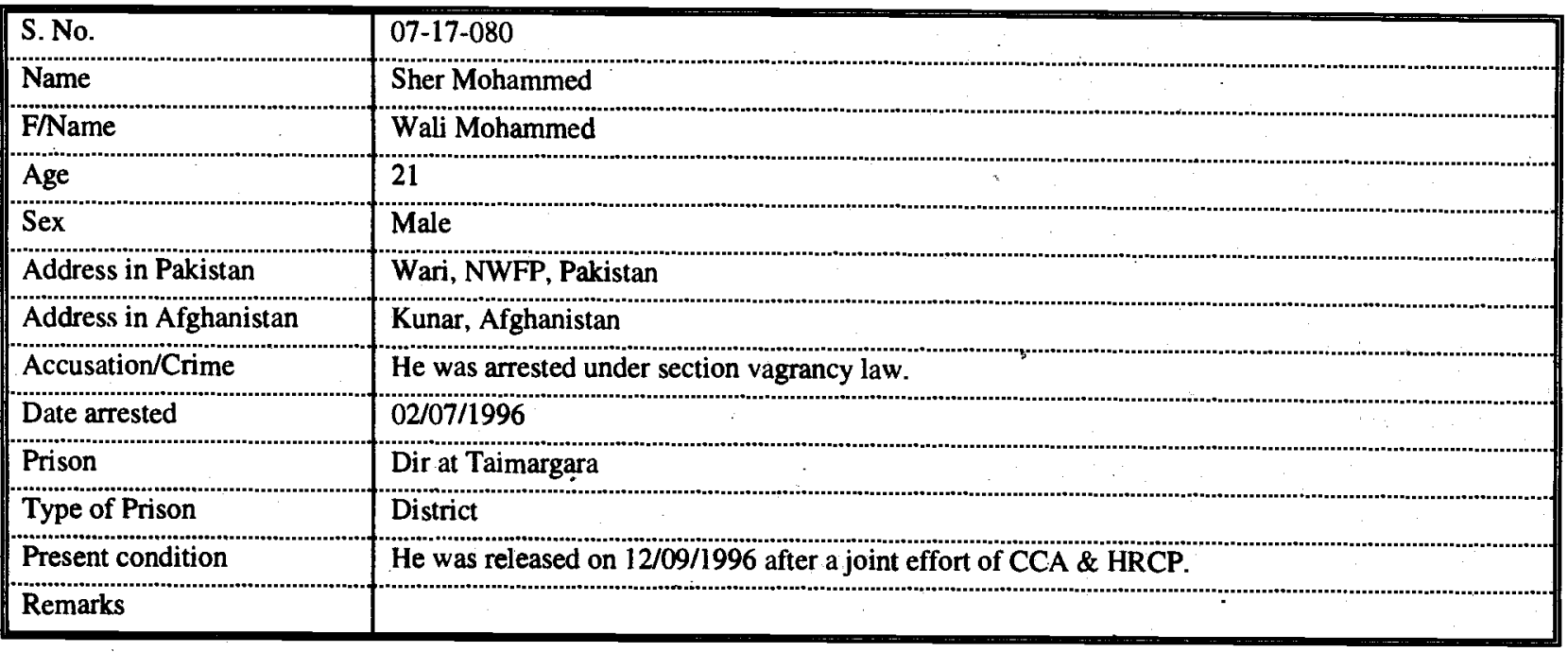

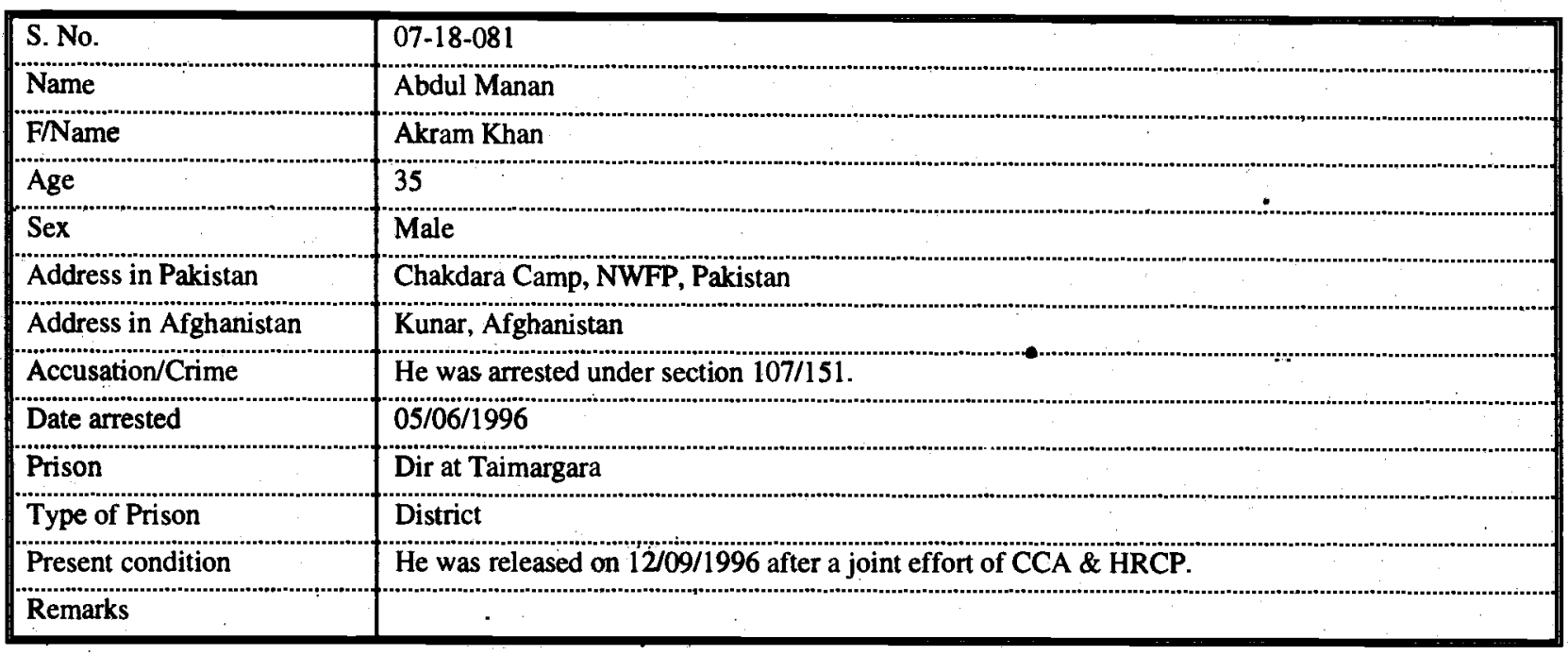

\begin{tabular}{|l|l|}
\hline S. No. & $07-19-082$ \\
\hline Name & Shah Wali \\
\hline F/Name & Mohammed Khan \\
\hline Age & 25 \\
\hline Sex & Male \\
\hline Address in Pakistan & Dir, NWFP, Pakistan \\
\hline Address in Afghanistan & Kunar, Afghanistan \\
\hline Accusation/Crime & He was arrested under section 4. \\
\hline Date arrested & Dir at Taimargara \\
\hline Prison & District \\
\hline Type of Prison & \\
\hline Present condition & \\
\hline Remarks & \\
\hline
\end{tabular}




\begin{tabular}{|c|c|}
\hline S. No. & $07-20-083$ \\
\hline Name & Nasib Khan \\
\hline F/Name & Fazli \\
\hline Age & 35 \\
\hline Sex & Male \\
\hline Address in Pakistan & Mayar Camp, NWFP, Pakistan \\
\hline Address in Afghanistan & Kunar, Afghanistan \\
\hline Accusation/Crime & He was arrested under section $107 / 151$. \\
\hline Date arrested & \\
\hline Prison & Dir at Taimargara \\
\hline Type of Prison & District \\
\hline Present condition & He was released on $13 / 11 / 1996$ after a joint effort of CCA \& HRCP. \\
\hline Remarks & \\
\hline
\end{tabular}

\begin{tabular}{|c|c|}
\hline S. No. & $07-21-084$ \\
\hline Name & Hamidullah \\
\hline F/Name & Rahat Jan \\
\hline Age & $28 \div \cdots$ \\
\hline Sex & Male \\
\hline Address in Pakistan & Mayar Camp, NWFP, Pakistan \\
\hline Address in Afghanistan & Kunar, Afghanistan \\
\hline Accusation/Crime & He was arrested under section 1071151 . \\
\hline Date arrested & (n) \\
\hline Prison & Dir at Taimargara \\
\hline Type of Prison & District \\
\hline Present condition & He was released on 13/11/1996 after a joint effort of CCA \& HRCP. \\
\hline Remarks & \\
\hline
\end{tabular}

\begin{tabular}{|c|c|}
\hline S. No. & $07-22-085$ \\
\hline Name & Zahir Shah \\
\hline F/Name & Mohammed Munir \\
\hline \multicolumn{2}{|l|}{ Age } \\
\hline Sex & Male \\
\hline Address in Pakistan & Mayar Camp, NWFP, Pakistan \\
\hline Address in Afghanistan & Kunar, Afghanistan \\
\hline Accusation/Crime & He was arrested under section $3 / 4$ (narcotics). \\
\hline \multicolumn{2}{|l|}{ Date arrested } \\
\hline Prison & Dir at Taimargara \\
\hline Type of Prison & District \\
\hline Present condition & He was released on 13/11/1996 after a joint effort of CCA \& HRCP. \\
\hline Remarks & \\
\hline
\end{tabular}




\begin{tabular}{|c|c|}
\hline S. No. & $07-23-086$ \\
\hline Name & Omar Gul \\
\hline F/Name & Abdul Qader \\
\hline \multicolumn{2}{|l|}{ Age } \\
\hline Sex & Male \\
\hline Address in Pakistan & Tor Camp, NWFP, Pakistan \\
\hline Address in Afghanistan & Kunar, Afghanistan \\
\hline Accusation/Crime & He was arrested under section $107 / 151$. \\
\hline \multicolumn{2}{|l|}{ Date arrested } \\
\hline Prison & Dir at Taimargara \\
\hline Type of Prison & (1) \\
\hline Present condition & He was released on 13/11/1996 after a joint effort of CCA \& HRCP. \\
\hline Remarks & \\
\hline
\end{tabular}

\begin{tabular}{|c|c|}
\hline S. No. & $07-24-087$ \\
\hline Name & Shakeruliah \\
\hline F/Name & Mohammed Jan \\
\hline \multicolumn{2}{|l|}{ Age } \\
\hline Sex & Male \\
\hline Address in Pakistan & Mayar Camp, NWFP, Pakistan \\
\hline Address in Afghanistan & Kunar, Afghanistan \\
\hline Accusation/Crime & He was arrested under section $107 / 151$. \\
\hline \multicolumn{2}{|l|}{ Date arrested } \\
\hline Prison & Dir at Taimargara \\
\hline Type of Prison & District \\
\hline Present condition & He was released on 13/11/1996 after a joint effort of CCA \& HRCP. \\
\hline Remarks & \\
\hline
\end{tabular}

\begin{tabular}{|c|c|}
\hline S. No. & $07-25-088$ \\
\hline Name & Inzar Gul \\
\hline F/Name & Sayyed Omar \\
\hline \multicolumn{2}{|l|}{ Age } \\
\hline Sex & Male \\
\hline Address in Pakistan & Mayar Camp, NWFP, Pakistan \\
\hline Address in Afghanistan & Kunar, Afghanistan \\
\hline Accusation/Crime & He was arrested under section $107 / 151$. \\
\hline \multicolumn{2}{|l|}{ Date arrested } \\
\hline Prison & Dir at Taimargara \\
\hline Type of Prison & District \\
\hline Present condition & He was released on $13 / 11 / 1996$ after a joint effort of CCA \& HRCP. \\
\hline Remarks & \\
\hline
\end{tabular}




\begin{tabular}{|c|c|}
\hline S. No. & 07-26-089 \\
\hline Name & Amanullah \\
\hline F/Name & Akbar Khan \\
\hline \multicolumn{2}{|l|}{ Age } \\
\hline Sex & Male \\
\hline Address in Pakistan & Mayar Camp, NWFP, Pakistan \\
\hline Address in Afghanistan & Kunar, Afghanistan \\
\hline Accusation/Crime & He was arrested under section $107 / 151$. \\
\hline \multicolumn{2}{|l|}{ Date arrested } \\
\hline Prison & Dir at Taimargara \\
\hline Type of Prison & District \\
\hline Present condition & He was released on $13 / 11 / 1996$ after a joint effort of CCA \& HRCP. \\
\hline Remarks & . \\
\hline
\end{tabular}

\begin{tabular}{|c|c|}
\hline S: No. & $07-27-090$ \\
\hline Name & Sherzada \\
\hline F/Name & Niaz Mohammed Khan \\
\hline Age & ? \\
\hline Sex & Male \\
\hline Address in Pakistan & Chakdara Camp, NWFP, Pakistan \\
\hline Address in Afghanistan & Kunar, Afghanistan \\
\hline Accusation/Crime & He was arrested under section $107 / 151$. \\
\hline Date arrested & 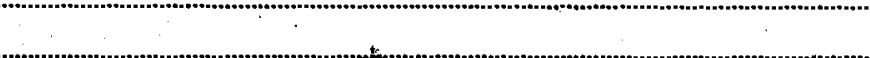 \\
\hline Prison - & Dir at Taimargara \\
\hline Type of Prison & District \\
\hline Present condition & He was released on $13 / 11 / 1996$ after a joint effort of CCA \& HRCP. \\
\hline Remarks & \\
\hline
\end{tabular}




\section{Central Prison Harinur}

\begin{tabular}{|l|l|l|}
\hline S. No. & 08-01-091 \\
\hline Name & Gul Zar & Khandar \\
\hline F/Name & \\
\hline Age & Male \\
\hline Sex & Gul Bahar, Peshawar, NWFP, Pakistan \\
\hline Address in Pakistan & Kunar, Afghanistan \\
\hline Address in Afghanistan & He was arrested under section 4PO. \\
\hline Accusation/Crime & $02 / 03 / 1996$ \\
\hline Date arrested & Haripur \\
\hline Prison & Central Prison \\
\hline Type of Prison & Not released. \\
\hline Present condition & $\vdots$ \\
\hdashline Remarks &
\end{tabular}




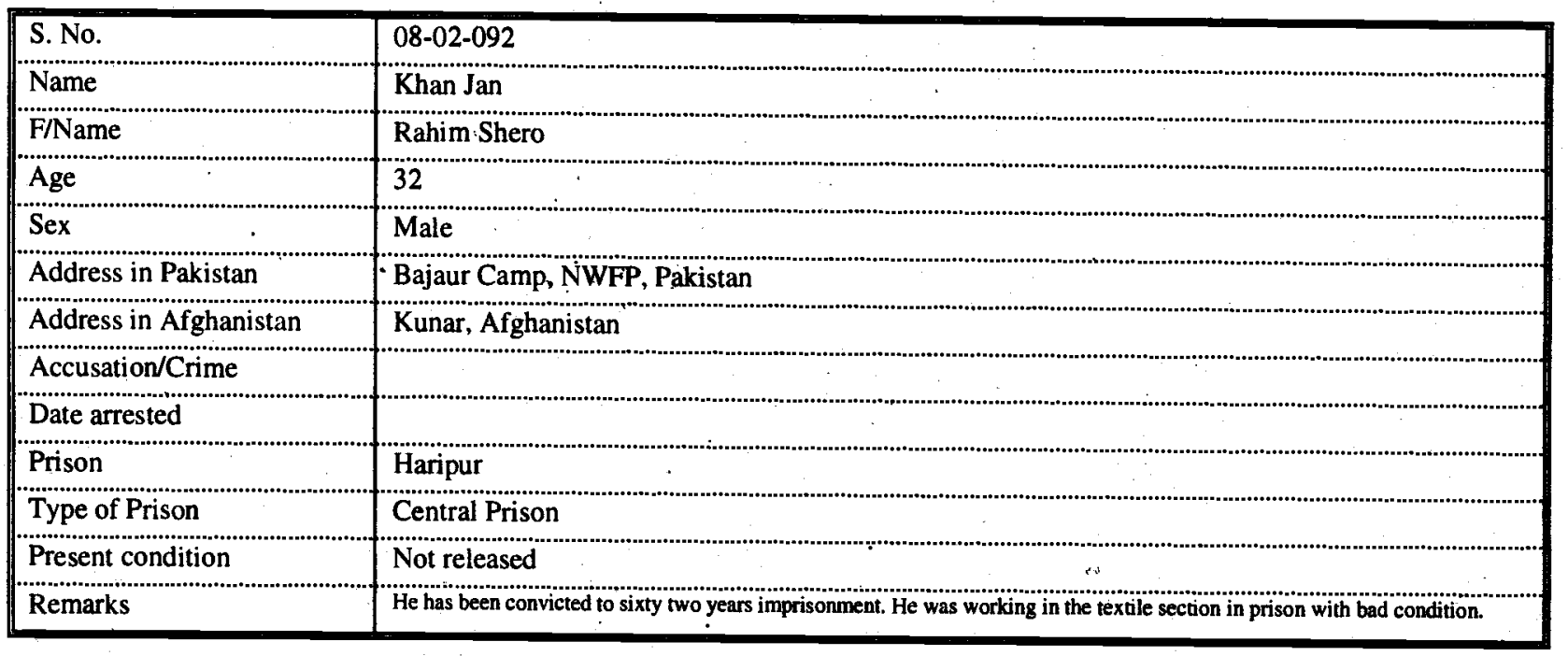

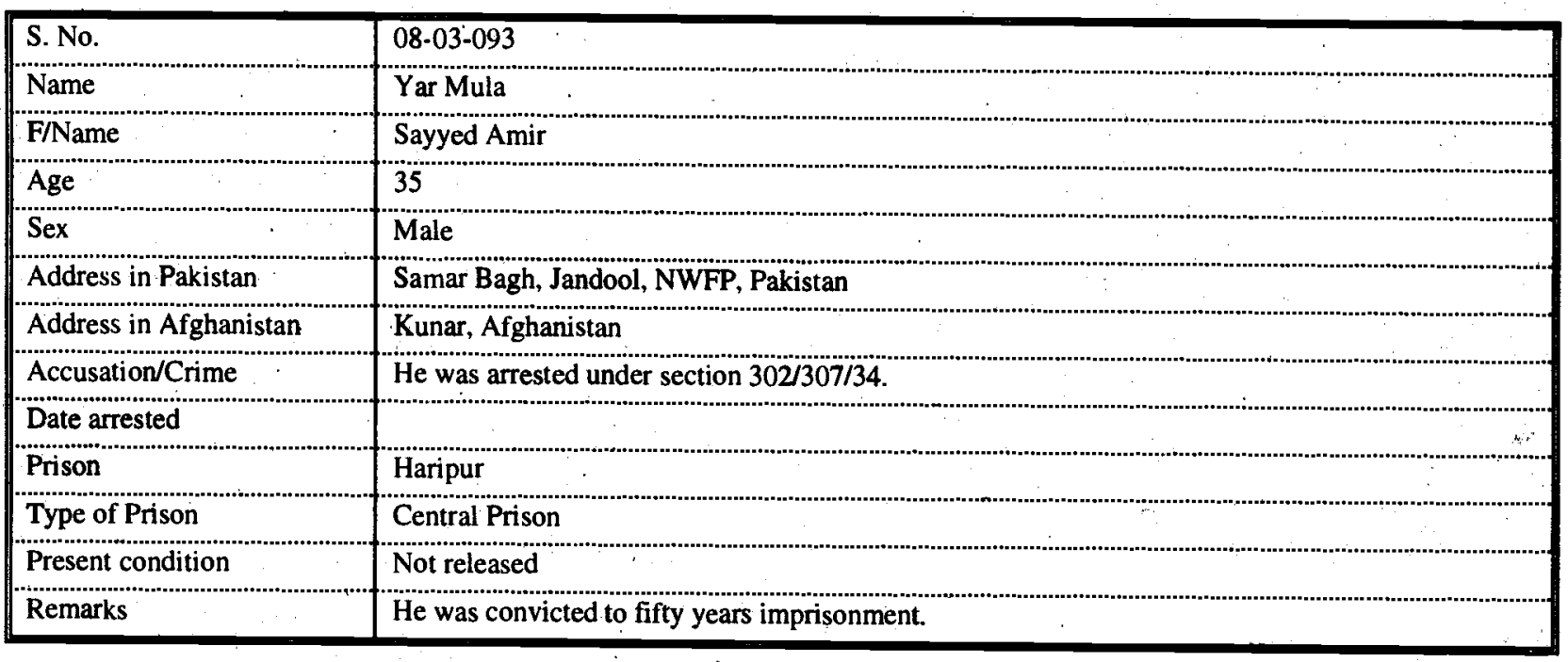

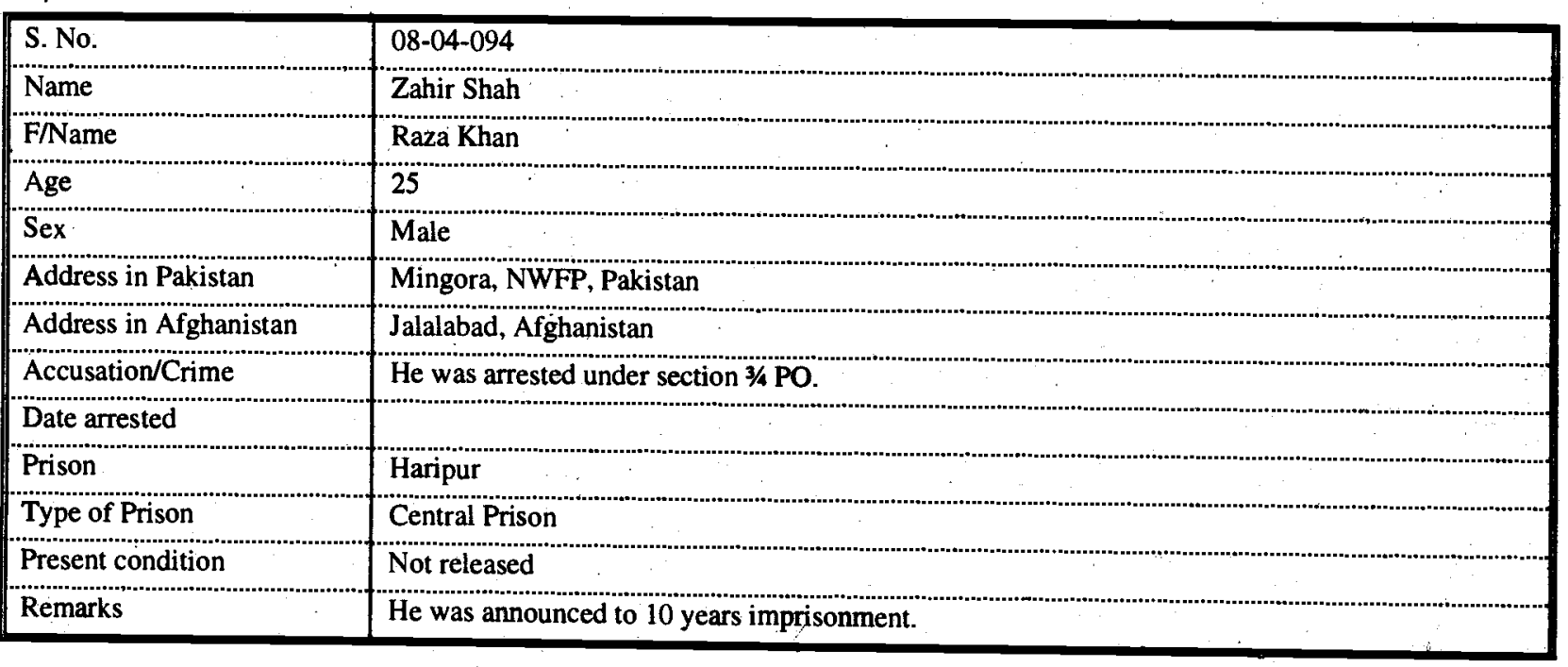




\begin{tabular}{|c|c|}
\hline S. No. & 08-05-095 \\
\hline Name & Sultan Mohammed \\
\hline F/Name & Gul Mohammed \\
\hline Age & 30 \\
\hline Sex & Male \\
\hline Address in Pakistan & Mingora, NWFP, Pakistan \\
\hline Address in Afghanistan & Jalalabad, Afghanistan \\
\hline Accusation/Crime & He was arrested under section $302 / 334$. \\
\hline \multicolumn{2}{|l|}{ Date arrested } \\
\hline Prison & Haripur \\
\hline Type of Prison & Central Prison \\
\hline Present condition & Not released. \\
\hline Remarks & He was announced 29 years imprisonment. \\
\hline
\end{tabular}

\begin{tabular}{|l|l|}
\hline S. No. & $08-06-096$ \\
\hline Name & Fazali Mahmood \\
\hline F/Name & Gul Mahmood \\
\hline Age & 30 \\
\hline Sex & Male \\
\hline Address in Pakistan & Mingora, NWFP, Pakistan \\
\hline Address in Afghanistan & Jalalabad, Afghanistan \\
\hline Accusation/Crime & He was arrested under section 302/334. \\
\hline Date arrested & Haripur \\
\hline Prison & Central Prison \\
\hline Type of Prison & Not released \\
\hline Present condition & He was announced 29 years imprisonment. \\
\hline Remarks &
\end{tabular}

\begin{tabular}{|c|c|}
\hline S. No. & $08-07-097$ \\
\hline Name & Mohammed Jan \\
\hline F/Name & Yama Gul \\
\hline Age & 40 \\
\hline Sex & Male \\
\hline Address in Pakistan & Gundoos, NWFP, Pakistan \\
\hline Address in Afghanistan & (1) \\
\hline Accusation/Crime & He was arrested under section $302 / 334$. \\
\hline Date arrested & $\ldots$ \\
\hline Prison & Haripur \\
\hline Type of Prison & Central Prison \\
\hline Present condition & Not released \\
\hline Remarks & His case was decided by session court Dir announced 25 years imprisonment \\
\hline
\end{tabular}




\begin{tabular}{|c|c|}
\hline S. No. & $08-08-098$ \\
\hline Name & Usman Ghani \\
\hline F/Name & Khaista Gul \\
\hline Age & 37 \\
\hline Sex & Male \\
\hline \multicolumn{2}{|l|}{ Address in Pakistan } \\
\hline Address in Afghanistan & Jalalabad, Afghanistan \\
\hline Accusation/Crime & He was arrested under section 324 . \\
\hline \multicolumn{2}{|l|}{ Date arrested } \\
\hline Prison & Haripur \\
\hline Type of Prison & Central Prison \\
\hline Present condition & Not released \\
\hline Remarks & He was announced 10 years imprisonment. \\
\hline
\end{tabular}

\begin{tabular}{|c|c|}
\hline S. No. & 08-09-099 \\
\hline Name & Ghulam Nabi \\
\hline Finame & Rahmatullah \\
\hline Age & 26 \\
\hline Sex & Male \\
\hline Address in Pakistan & Kachi Garhi Camp, NWFP, Pakistan \\
\hline Address in Afghanistan & Kandahar, Afghanistan \\
\hline Accusation/Crime & He was arrested under section 364 . \\
\hline \multicolumn{2}{|l|}{ Date arrested. } \\
\hline Prison & Haripur \\
\hline Type of Prison & Central Prison \\
\hline Present condition & Not released. \\
\hline Remarks & He was announced 10 years imprisonment. \\
\hline
\end{tabular}

\begin{tabular}{|c|c|}
\hline S. No. & $08-10-100$ \\
\hline Name & Abdul Manan \\
\hline F/Name & Said Amin \\
\hline Age & 40 \\
\hline Sex & Male \\
\hline Address in Pakistan & Qandoos, NWFP, Pakistan \\
\hline \multicolumn{2}{|c|}{ Address in Afghanistan } \\
\hline Accusation/Crime & He was arrested under section 302 . \\
\hline \multicolumn{2}{|l|}{ Date arrested } \\
\hline Prison & Haripur \\
\hline Type of Prison & Central Prison \\
\hline Present condition & Not released. \\
\hline Remarks & He has been convicted to 25 years imprisonment. \\
\hline
\end{tabular}




\begin{tabular}{|c|c|}
\hline S. No. & $08-11-101$ \\
\hline Name & Aziz \\
\hline FName & Painda Khan \\
\hline Age & 38 \\
\hline Sex & Male \\
\hline Address in Pakistan & Taimargara Camp, NWFP, Pakistan \\
\hline Address in Afghanistan & Kunar, Afghanistan \\
\hline Accusation/Crime & He was arrested under section $3 / 4 \mathrm{PO}$. \\
\hline Date arrested & \\
\hline Prison & Haripur \\
\hline Type of Prison & Central Prison \\
\hline Present condition. & Not released. \\
\hline Remarks & He has been announced 12 years imprisonment. \\
\hline
\end{tabular}

\begin{tabular}{|l|}
\hline S. No. \\
\hline Name
\end{tabular}

\section{8-12-102}

Gul Amir

F/Name

Age

Sex

Said Amir

20

Male

Address in Pakistan

Address in Afghanistan

Accusation/Crime

Date arrested

Prison

Type of Prison

Present condition

Chak Dara, NWFP, Pakistan

Kunar, Afghanistan

He was arrẹsted under section 302 .

Remarks

Haripur

Central Prison

Not released.

He has been announced 25 years imprisonment.

\begin{tabular}{|c|c|}
\hline S. No. & $08-13-103$ \\
\hline Name & Gul Mula \\
\hline F/Name & said Amir \\
\hline Age & 20 \\
\hline Sex & Male \\
\hline Address in Pakistan & Chak Dara, NWFP, Pakistan \\
\hline Address in Afghanistan & Kunar, Afghanistan \\
\hline Accusation/Crime & He was arrested under section 302 . \\
\hline \multicolumn{2}{|l|}{ Date arrested } \\
\hline Prison & Haripur \\
\hline Type of Prison & Central Prison \\
\hline Present condition & Not released. \\
\hline Remarks & He has been announced 25 years imprisonment. \\
\hline
\end{tabular}




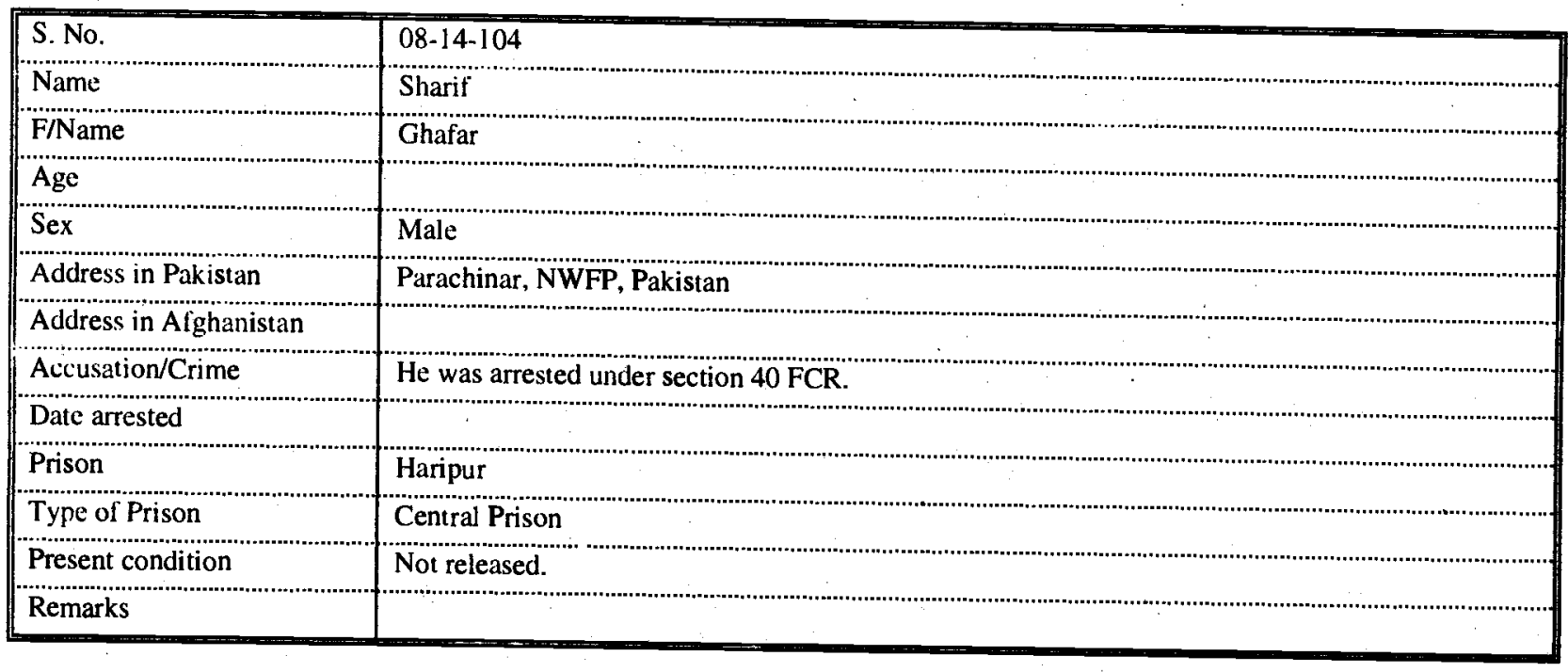




\section{Distrigt Prisen Kohat}

\begin{tabular}{|c|c|}
\hline S. No. & $09-001-105$ \\
\hline Name & Sher Gul \\
\hline F/Name & Bunir Gul \\
\hline Age & 25 \\
\hline Sex & Male \\
\hline Address in Pakistan & Bagan Camp, Parachinar, Pakistan \\
\hline \multicolumn{2}{|c|}{ Address in Afghanistan } \\
\hline Accusation/Crime & He was arrested under section $40 \mathrm{FCR}$. \\
\hline \multicolumn{2}{|l|}{ Date arrested } \\
\hline Prison & Kohat \\
\hline Type of Prison & District Prison \\
\hline Present condition & Not released. \\
\hline Remarks & He has been announced 1 year imprisonment. \\
\hline
\end{tabular}




\begin{tabular}{|c|c|}
\hline S. No. & 09-002-106 \\
\hline Name & Sayyed Akbar \\
\hline F/Name & Shamsher \\
\hline Age & 30 \\
\hline Sex & Male \\
\hline Address in Pakistan & Bagan Camp, Parachinar, Pakistan \\
\hline \multicolumn{2}{|c|}{ Address in Afghanistan } \\
\hline Accusation/Crime & He was arrested under section 40 FCR. \\
\hline \multicolumn{2}{|l|}{ Date arrested } \\
\hline Prison & Kohat \\
\hline Type of Prison & District Prison \\
\hline Present condition & Not released." \\
\hline Remarks & He has been announced 1 year imprisonment. \\
\hline
\end{tabular}

\begin{tabular}{|c|c|}
\hline S. No. & 09-003-107 \\
\hline Name & Fazal Mehmood \\
\hline F/Name & Yar Mehmood \\
\hline Age & 25 \\
\hline Sex & Male \\
\hline Address in Pakistan & Dawaba Camp, NWFP, Pakistan \\
\hline Address in Afghanistan & Paktia, Afghanistan \\
\hline Accusation/Crime & He was arrested under section 40 FCR. \\
\hline \multicolumn{2}{|l|}{ Date arrested } \\
\hline Prison & Kohat \\
\hline Type of Prison & District Prison \\
\hline Present condition & Not released. \\
\hline Remarks & \\
\hline
\end{tabular}

\begin{tabular}{|c|c|}
\hline S. No. & 09-004-108 \\
\hline Name & Habib \\
\hline F/Name & Ajab Gul \\
\hline Age & 65 \\
\hline Sex & Male \\
\hline Address in Pakistan & Parachinar. NWFP, Pakistan \\
\hline Address in Afghanistan & Paktia, Afghanistan \\
\hline Accusation/Crime & He was arrested under section $40 \mathrm{FCR}$. \\
\hline \multicolumn{2}{|l|}{ Date arrested } \\
\hline Prison & Kohat \\
\hline Type of Prison & District Prison \\
\hline Present condition & Not released. \\
\hline Remarks & \\
\hline
\end{tabular}




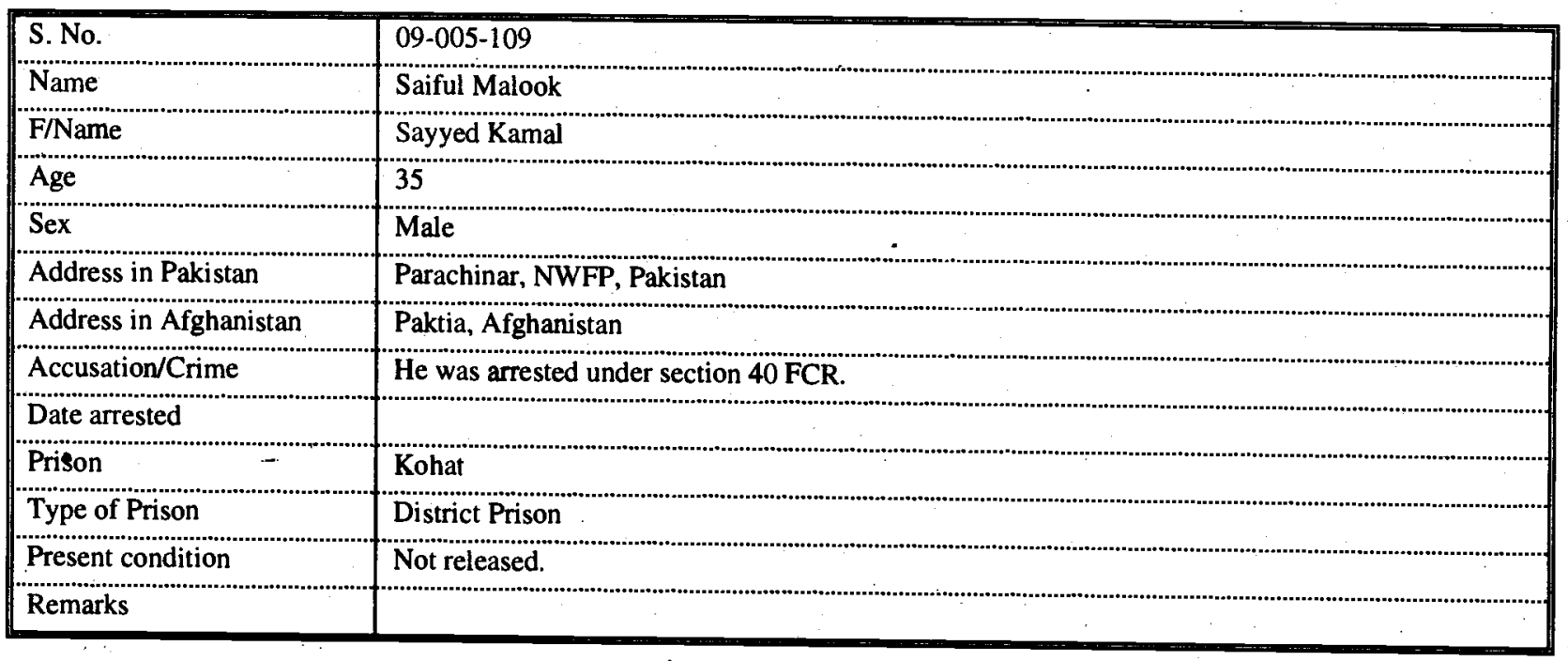

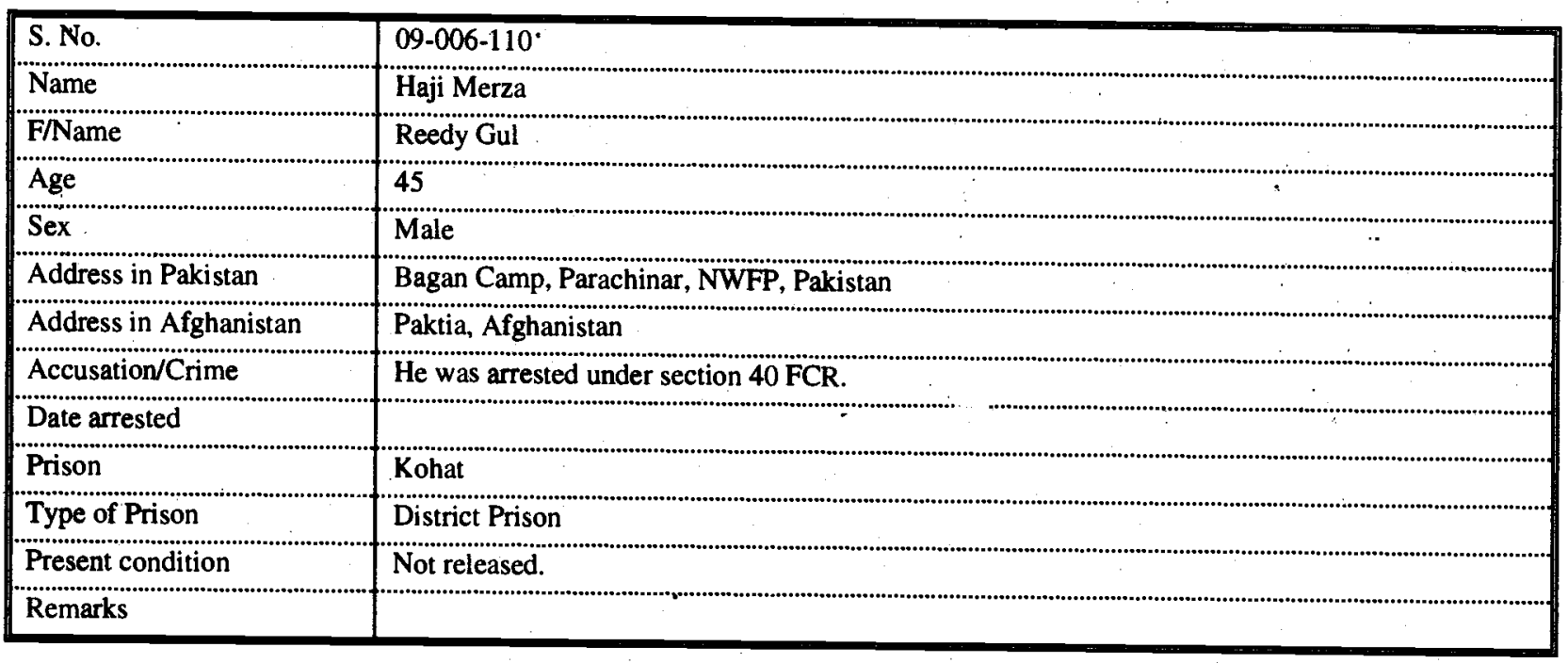

\begin{tabular}{|c|c|}
\hline S. No. & $09-007-111$ \\
\hline Name & Sabat \\
\hline F/Name & Hassan Gul \\
\hline Age & 65 \\
\hline Sex & Male \\
\hline Address in Pakistan & Bagan Camp, Parachinar; NWFP, Pakistan \\
\hline Accusation/Crime & He was arrested under section 40 FCR. \\
\hline \multicolumn{2}{|l|}{ Date arrested } \\
\hline Prison & (1) \\
\hline Type of Prison & (n) \\
\hline
\end{tabular}




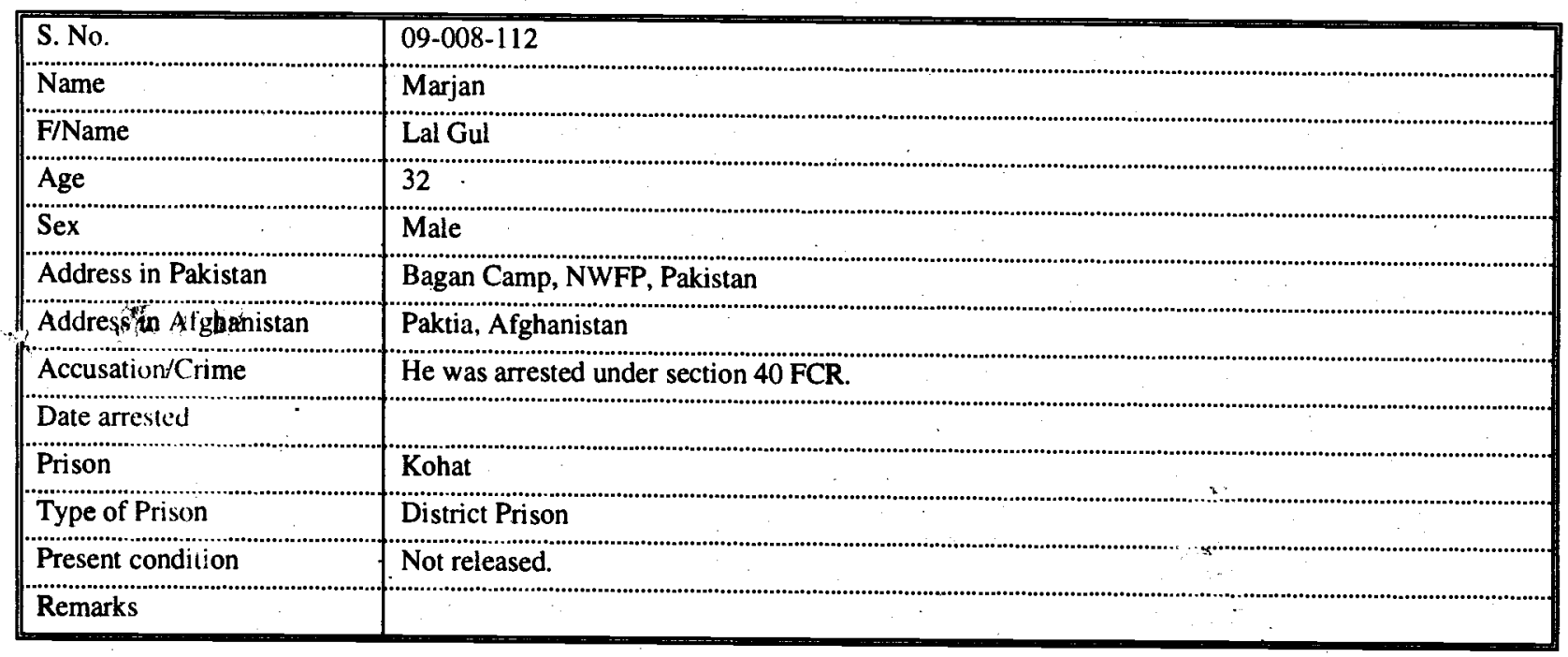

\begin{tabular}{|c|c|}
\hline S. No. & $09-009-113$ \\
\hline Name & Mirwais \\
\hline F/Name & Saadat Khan \\
\hline Age & 41 \\
\hline Sex & Male \\
\hline Address in Pakistan & Bagan Camp, Paranchinar, NWFP, Pakistan \\
\hline Address in Afghanistan & Paktia, Afghanistan \\
\hline Accusation/Crime & He was arrested under section 40 FCR. \\
\hline \multicolumn{2}{|l|}{ Date arrested } \\
\hline Prison & Kohat \\
\hline Type of Prison & District Prison \\
\hline Present condition & Not released. \\
\hline Remarks & 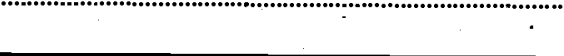 \\
\hline
\end{tabular}

\begin{tabular}{|c|c|}
\hline S. No. & $09-010-114$ \\
\hline Name & Samiullah \\
\hline F/Name & Gul Sadam \\
\hline \multicolumn{2}{|l|}{ Age } \\
\hline Sex & Male \\
\hline Address in Pakistan & Bagan Camp, Parachinar, NWFP, Pakistan \\
\hline Address in Afghanistan & Khost, Afghanistan \\
\hline Accusation/Crime & He was arrested under section $40 \mathrm{FCR}$. \\
\hline \multicolumn{2}{|l|}{ Date arrested } \\
\hline Prison & Kohat \\
\hline Type of Prison & District Prison \\
\hline Present condition & Not released. \\
\hline Remarks & \\
\hline
\end{tabular}




\begin{tabular}{|c|c|}
\hline S. No. & $09-011-115$ \\
\hline Vame & Sher Khan \\
\hline F/Name & Amin \\
\hline Age & 33 \\
\hline Sex & Male \\
\hline Address in Pakistan & Tal Bagan Camp, NWFP, Pakistan \\
\hline Address in Afghanistan & Afghanistan \\
\hline Accusation/Crime & He was arrested under section 179 PPC. \\
\hline \multicolumn{2}{|l|}{ Date arrested } \\
\hline Prison & Kohat \\
\hline Type of Prison & District Prison \\
\hline Present condition & Not released. \\
\hline Remarks & \\
\hline
\end{tabular}

\begin{tabular}{|c|c|}
\hline S. No. & $09-012-116$ \\
\hline Name & Amanullah \\
\hline F/Name & Bismullah \\
\hline Age & 30 \\
\hline Sex & Male \\
\hline Address in Pakistan & Kohat Camp, NWFP, Pakistan \\
\hline Address in Afghanistan & Khost, Afghanistan \\
\hline Accusation/Crime & He was arrested under section 40 FCR. \\
\hline \multicolumn{2}{|l|}{ Date arrested } \\
\hline Prison & Kohat \\
\hline Type of Prison & District Prison \\
\hline Present condition & Not released. \\
\hline Remarks & \\
\hline
\end{tabular}

\begin{tabular}{|c|c|}
\hline S. No. & $09-013-117$ \\
\hline Name & Pito Khan \\
\hline F/Name & Nazim \\
\hline \multicolumn{2}{|l|}{ Age } \\
\hline Sex & Male \\
\hline Address in Pakistan & Bagan Camp, Parachinar, NWFP, Pakistan \\
\hline Address in Afghanistan & Khost, Afghanistan \\
\hline Accusation/Crime & He was arrested under section 40 FCR. \\
\hline \multicolumn{2}{|l|}{ Date arrested } \\
\hline Prison & Kohat \\
\hline Type of Prison & Discist Prison \\
\hline Present condition & Noi released. \\
\hline Remarks & \\
\hline
\end{tabular}




\begin{tabular}{|c|c|}
\hline S. No. & $\begin{array}{c}09-014-118 \\
09-14\end{array}$ \\
\hline Name & Marjan \\
\hline F/Name & Piou Khan \\
\hline \multicolumn{2}{|l|}{ Age } \\
\hline Sex & Male \\
\hline Address in Pakistan & Dawaba, NWFP, Pakistan \\
\hline Address in Afghanistan & Khost, Afghanistan \\
\hline Accusation/Crime & He was arrested under section $40 \mathrm{FCR}$. \\
\hline \multicolumn{2}{|l|}{ Date arrested } \\
\hline Prison & Kohat \\
\hline Type of Prison & District Prison \\
\hline Present condition & Not released. \\
\hline Remarks & \\
\hline
\end{tabular}

\begin{tabular}{|c|c|}
\hline S. No. & $09-015-119$ \\
\hline Name & Hazrat Khan \\
\hline F/Name & Gul Bat Khan \\
\hline Age & 35 \\
\hline Sex & Male \\
\hline Address in Pakistan & Bagan Camp, Parachinar, NWFP, Pakistan \\
\hline Address in Afghanistan & Paktia, Afghanistan \\
\hline Accusation/Crime & He was arrested under section 40 FCR. \\
\hline \multicolumn{2}{|l|}{ Date arrested } \\
\hline Prison & Kohat \\
\hline Type of Prison & District Prison \\
\hline Present condition & Not released. \\
\hline Remarks & \\
\hline
\end{tabular}

\begin{tabular}{|c|c|c|}
\hline S. No. & $09-016-120$ & \\
\hline Name & Gul Mohammed & \\
\hline F/Name & Mehraban & . \\
\hline Age & 38 & \\
\hline Sex & Male & \\
\hline Address in Pakistan & Bagan Camp, Parachinar, NWFP, Pakistan & \\
\hline Address in Afghanistan & Paktia, Afghanistan & \\
\hline Accusation/Crime & He was arrested under section $40 \mathrm{FCR}$. & 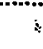 \\
\hline \multicolumn{3}{|l|}{ Date arrested } \\
\hline Prison & Kohat & \\
\hline Type of Prison & District Prison & \\
\hline Present condition & Not released. & \\
\hline Remarks & & \\
\hline
\end{tabular}




\begin{tabular}{|c|c|}
\hline S. No. & $09-017-121$ \\
\hline Name & Mohammed Nazir \\
\hline F/Name & Matna Khan \\
\hline Sex & Male \\
\hline Address in Pakistan & Ali Khail, Parachinar, NWFP, Pakistan \\
\hline Accusation/Crime & He was arrested under section 40 FCR. \\
\hline Date arrested & 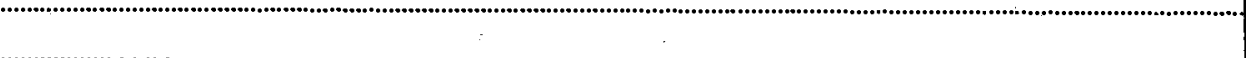 \\
\hline Prison & (1) \\
\hline Type of Prison & District Prison \\
\hline
\end{tabular}

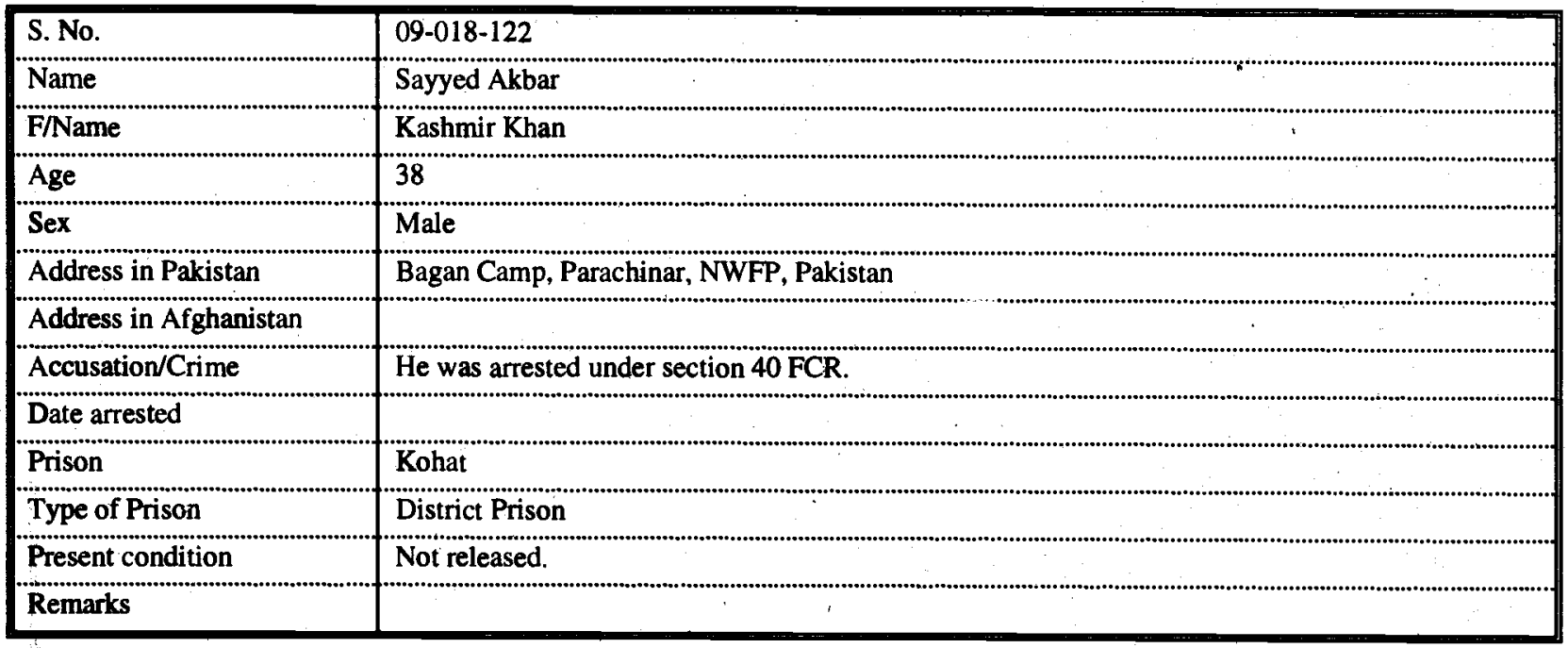

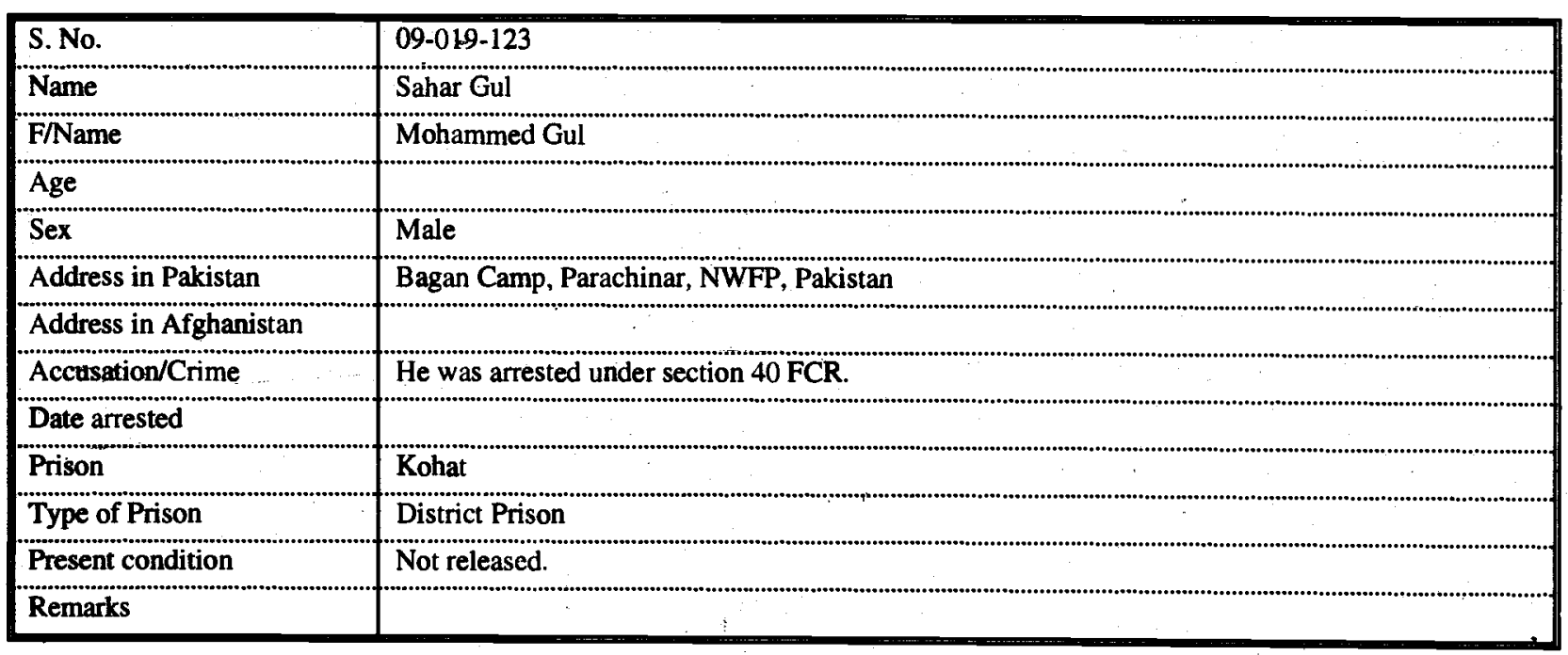




\begin{tabular}{|c|c|}
\hline S. No. & $09-020-124$ \\
\hline Name & Fazal Mahmood \\
\hline F/Name & Sher Mohmood \\
\hline Age & 47 \\
\hline Sex & Male \\
\hline Address in Pakistan & Dawaba Camp, NWFP, Pakistan \\
\hline Address in Afghanistan & Paktia, Afghanistan \\
\hline Accusation/Crime & He was arrested under section 40 FCR. \\
\hline \multicolumn{2}{|l|}{ Date arrested } \\
\hline Prison & Kohat \\
\hline Type of Prison & District Prison \\
\hline Present condition & Not released. \\
\hline Remarks & \\
\hline
\end{tabular}

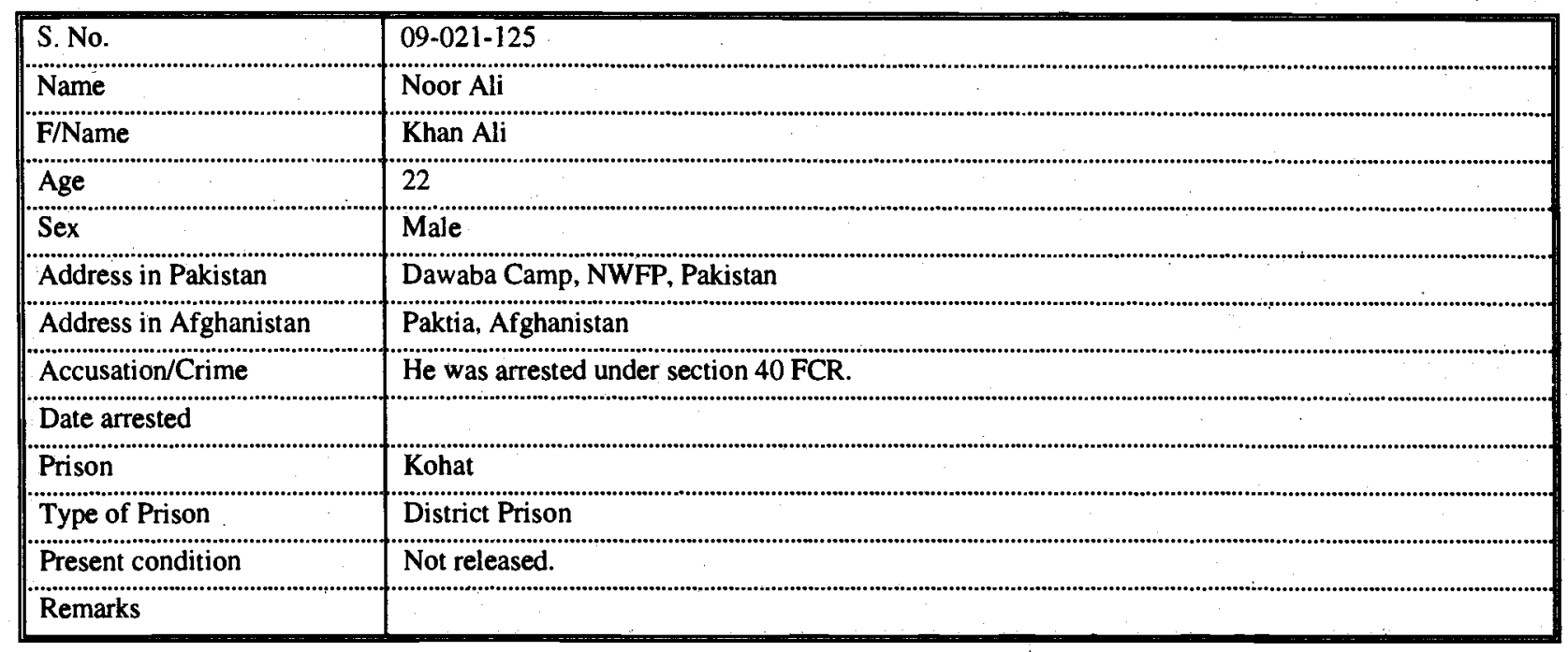

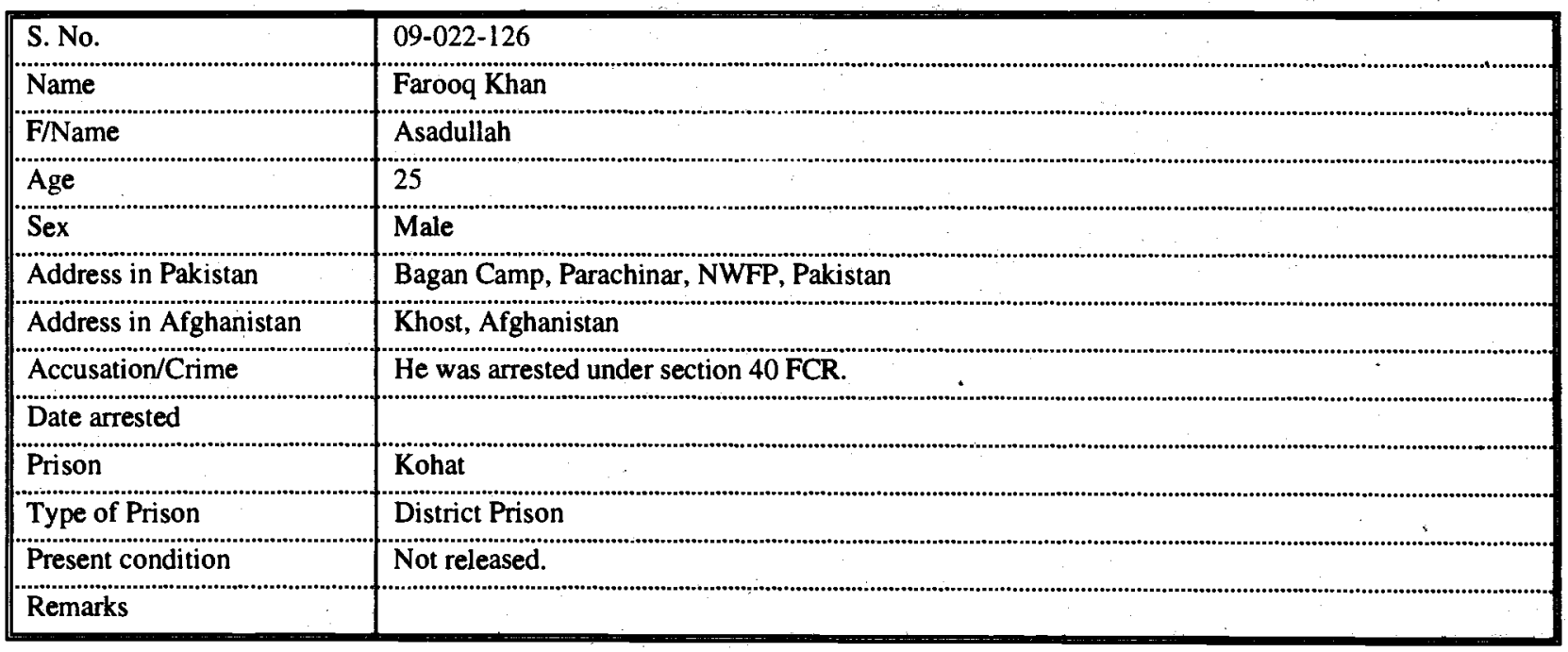




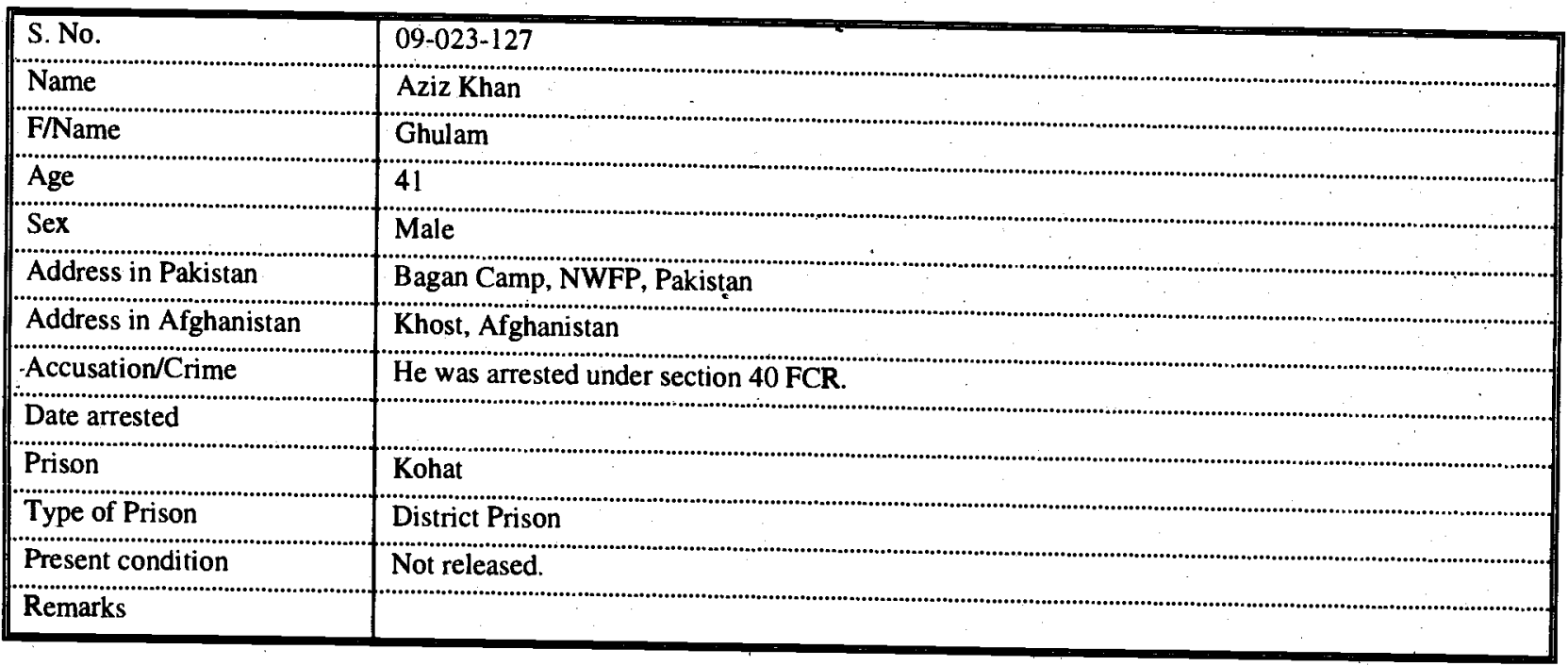

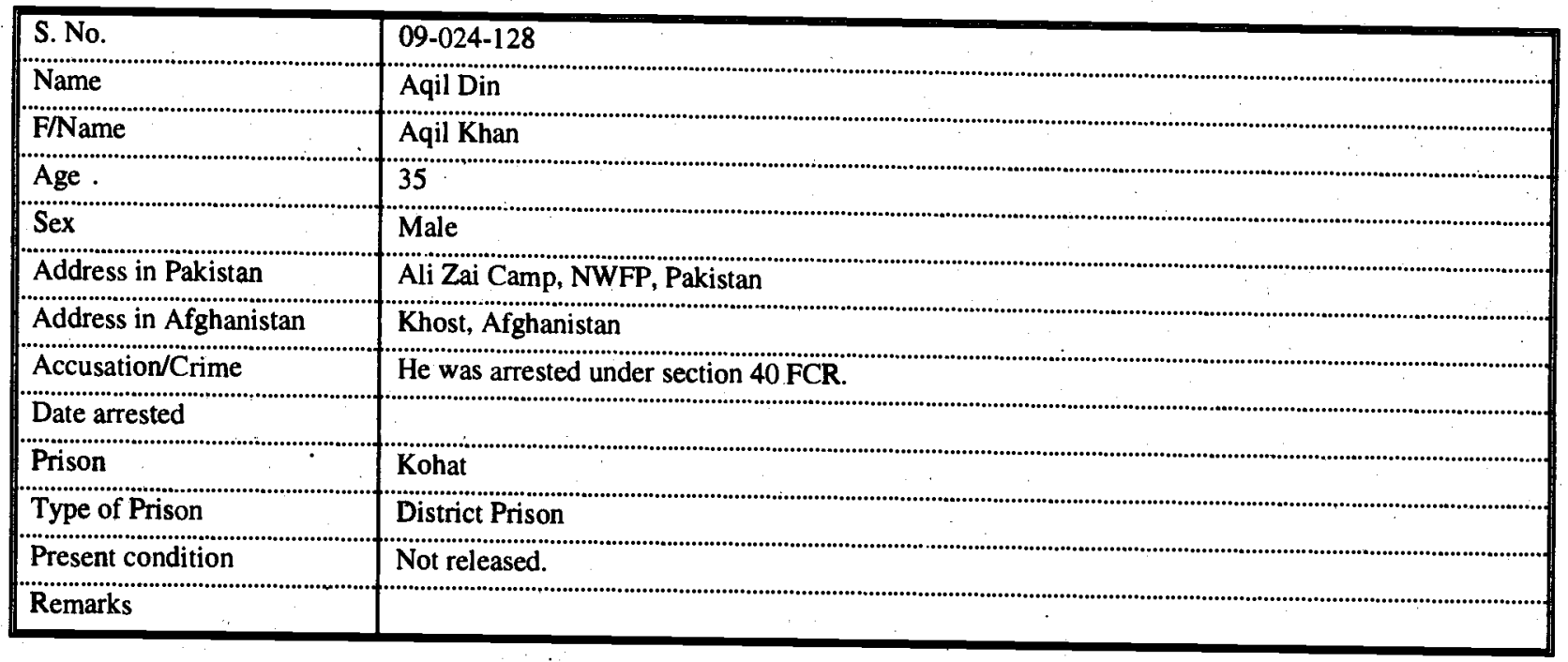

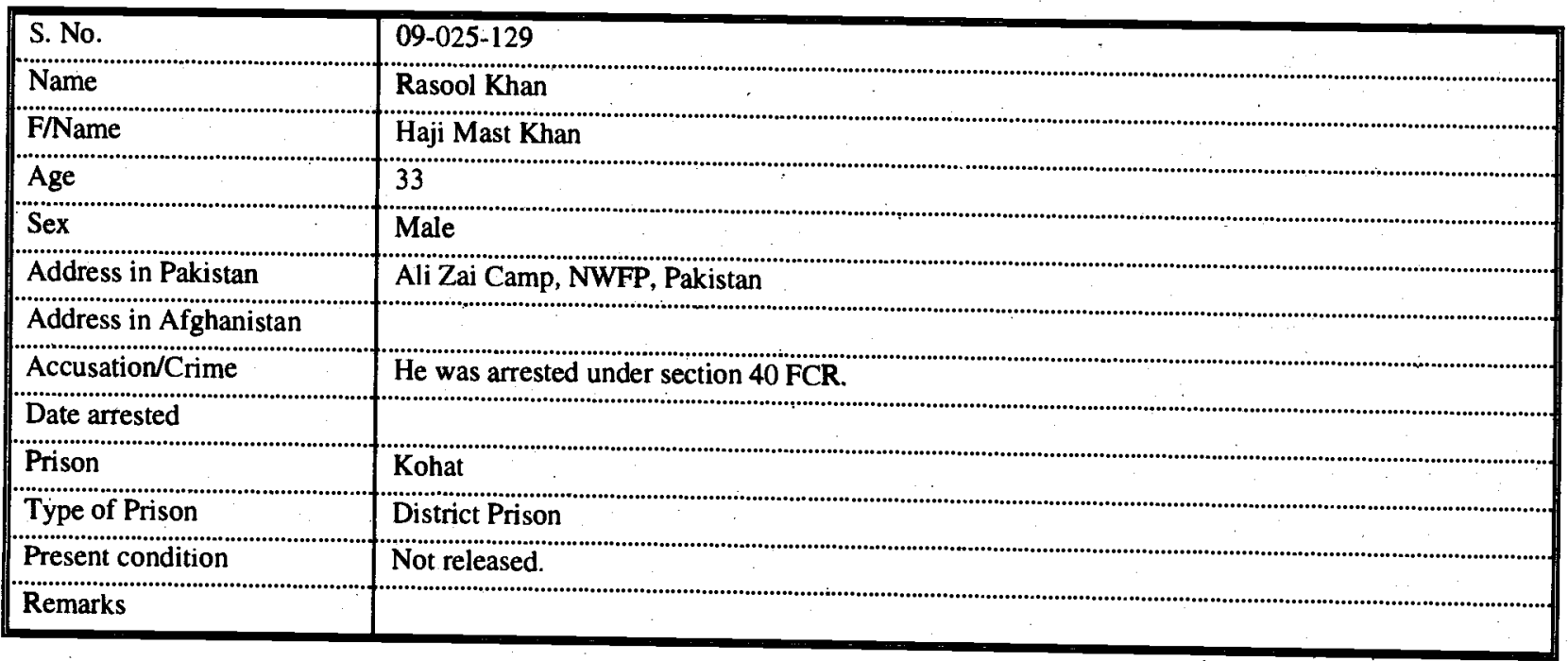




\begin{tabular}{|c|c|}
\hline S. No. & $09-026-130$ \\
\hline Name & Jan Mohammed \\
\hline F/Name & Noor Din \\
\hline \multicolumn{2}{|l|}{ Age } \\
\hline Sex & Male \\
\hline Address in Pakistan & Dawaba Camp, NWFP, Pakistan \\
\hline Address in Afghanistan & Khost, Afghanistan \\
\hline Accusation/Crime & He was arrested under section 40 FCR. \\
\hline \multicolumn{2}{|l|}{ Date arrested } \\
\hline Prison & Kohat \\
\hline Type of Prison & District Prison \\
\hline Present condirion & Not released. \\
\hline Remarks & \\
\hline
\end{tabular}

\begin{tabular}{|c|c|}
\hline S. No. & $09-027 \cdot 131$ \\
\hline Name & Noor Mohammed \\
\hline F/Name & Khail Mohammed \\
\hline \multicolumn{2}{|l|}{ Age } \\
\hline Sex & Male \\
\hline Address in Pakistan & Dawaba Camp, NWFP, Pakistan \\
\hline Address in Afghanistan & Paktia, Afghanistan \\
\hline Accusation/Crime & He was arrested under section 40 FCR. \\
\hline \multicolumn{2}{|l|}{ Date arrested } \\
\hline Prison & Kohat \\
\hline Type of Prison & District Prison \\
\hline Present condition & Not released. \\
\hline Remarks & \\
\hline
\end{tabular}

\begin{tabular}{|c|c|}
\hline S. No. & $09-028-132$ \\
\hline Name & Fazli Mahmood \\
\hline F/Name & Yar Mohmood \\
\hline Age & 30 \\
\hline Sex & Male \\
\hline Address in Pakistan & Dawaba Camp, NWFP, Pakistan \\
\hline Address in Afghanistan & Khost, Afghanistan \\
\hline 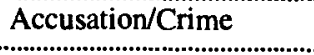 & He was arrested under section 40 FCR. \\
\hline \multicolumn{2}{|l|}{ Date arrested } \\
\hline Prison & Kohat \\
\hline Type of Prison & District Prison \\
\hline Present condition & Not released. \\
\hline Remarks & \\
\hline
\end{tabular}




\begin{tabular}{|c|c|}
\hline S. No. & $09-029-133$ \\
\hline Name & Saifullah \\
\hline F/Name & Bakht Jan \\
\hline Age & 33 \\
\hline Sex & Male \\
\hline Address in Pakistan & Sadda, NWFP, Pakistan \\
\hline Address in Afghanistan & Paktia, Afghanistan \\
\hline Accusation/Crime & He was arrested under section $40 \mathrm{FCR}$. \\
\hline Date arrested & \\
\hline Prison & Kohat \\
\hline Type of Prison & District Prison \\
\hline Present condition & Not released. \\
\hline Remarks & \\
\hline
\end{tabular}

\begin{tabular}{|c|c|}
\hline S. No. & $09-030-134$ \\
\hline Name & Mohammed Rahim \\
\hline F/Name & Haji Karim \\
\hline Age & 40 \\
\hline Sex & Male \\
\hline Address in Pakistan & Ali Zai Camp, NWFP, Pakistan \\
\hline Address in Afghanistan & Paktia, Afghanistan \\
\hline Accusation/Crime & He was arrested under section 40 FCR. \\
\hline \multicolumn{2}{|l|}{ Date arrested } \\
\hline Prison & Kohat \\
\hline Type of Prison & District Prison \\
\hline Present condition & Not released. \\
\hline Remarks & \\
\hline
\end{tabular}

\begin{tabular}{|c|c|}
\hline S. No. & $09-031-135$ \\
\hline Name & Hukam Jan \\
\hline F/Name & Bismillah \\
\hline Age & 35 \\
\hline Sex & Male \\
\hline Address in Pakistan & Ali Zai Bagan Camp, NWFP, Pakistan \\
\hline Address in Afghanistan & Paktia, Afghanistan \\
\hline Accusation/Crime & He was arrested under section 40 FCR. \\
\hline \multicolumn{2}{|l|}{ Date arrested } \\
\hline Prison & Kohat \\
\hline Type of Prison & District Prison \\
\hline Present condition & Not released. \\
\hline Remarks & \\
\hline
\end{tabular}




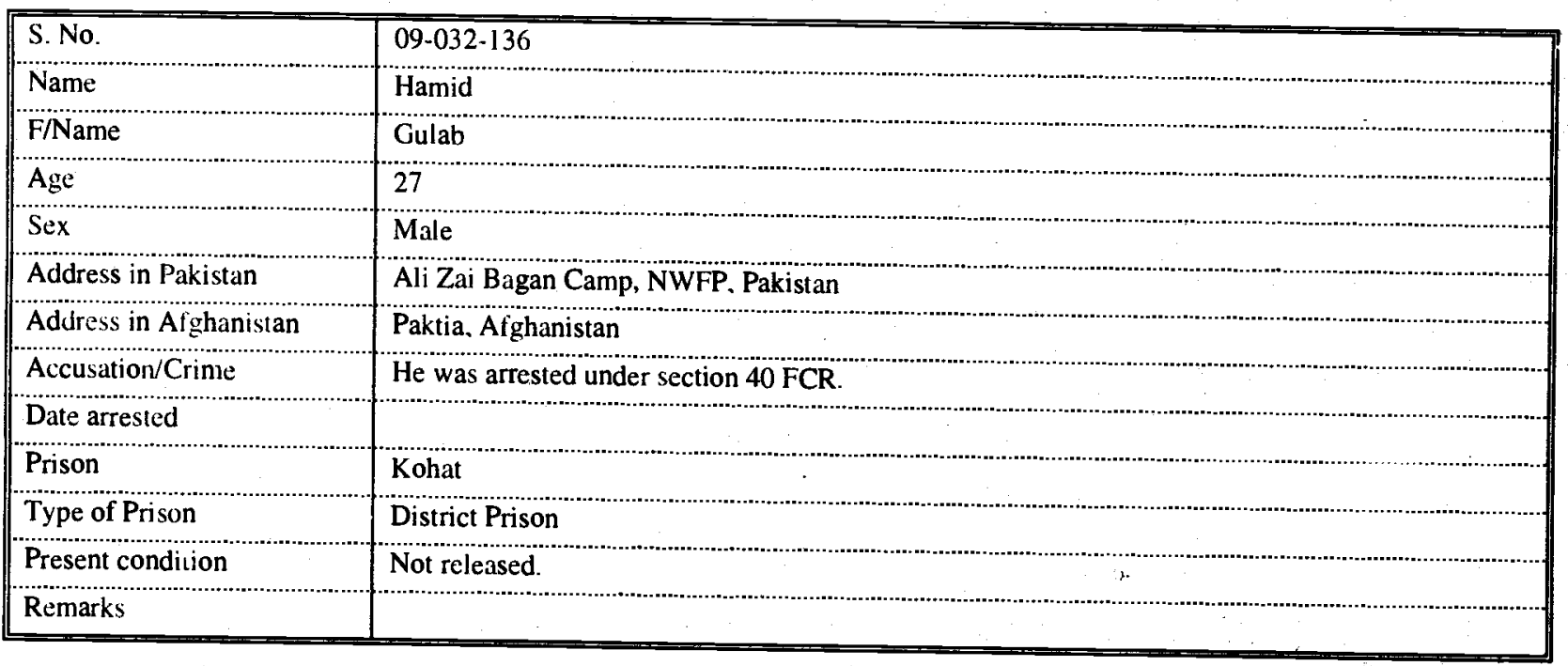

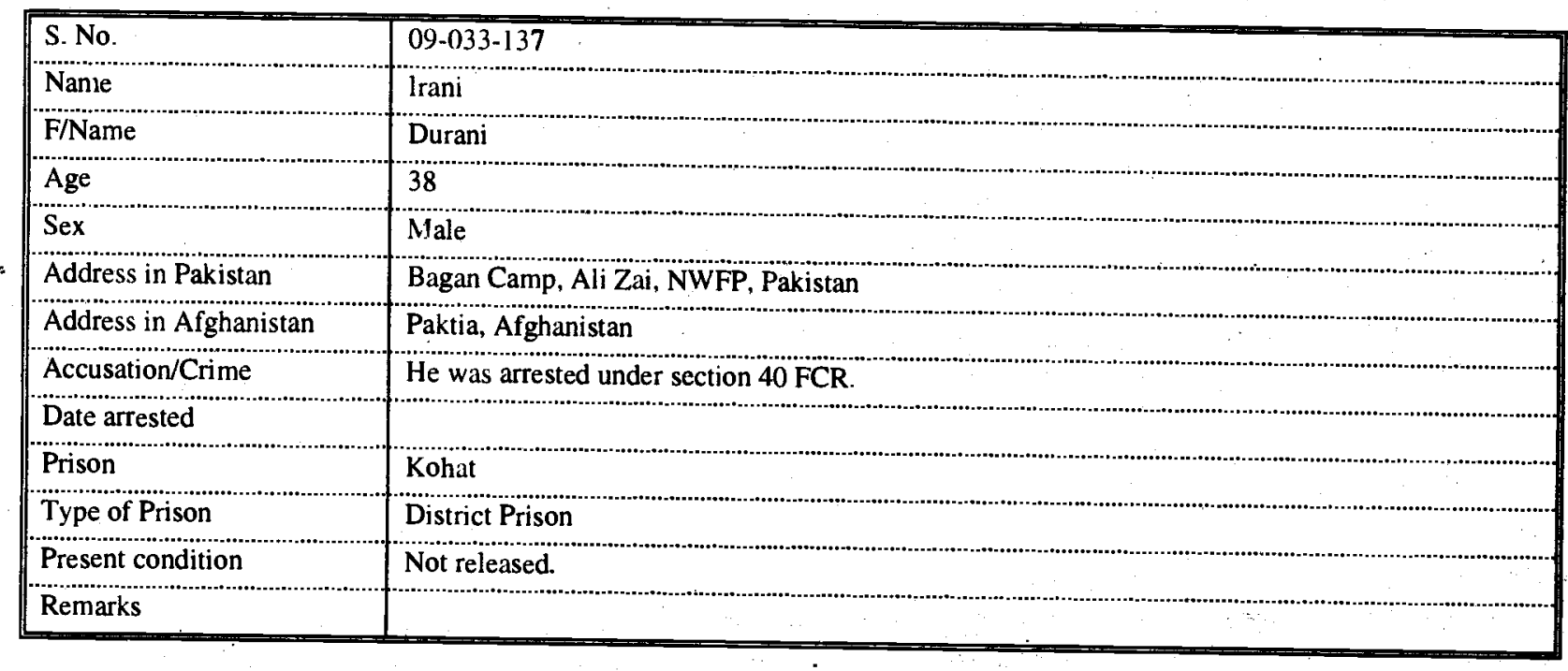

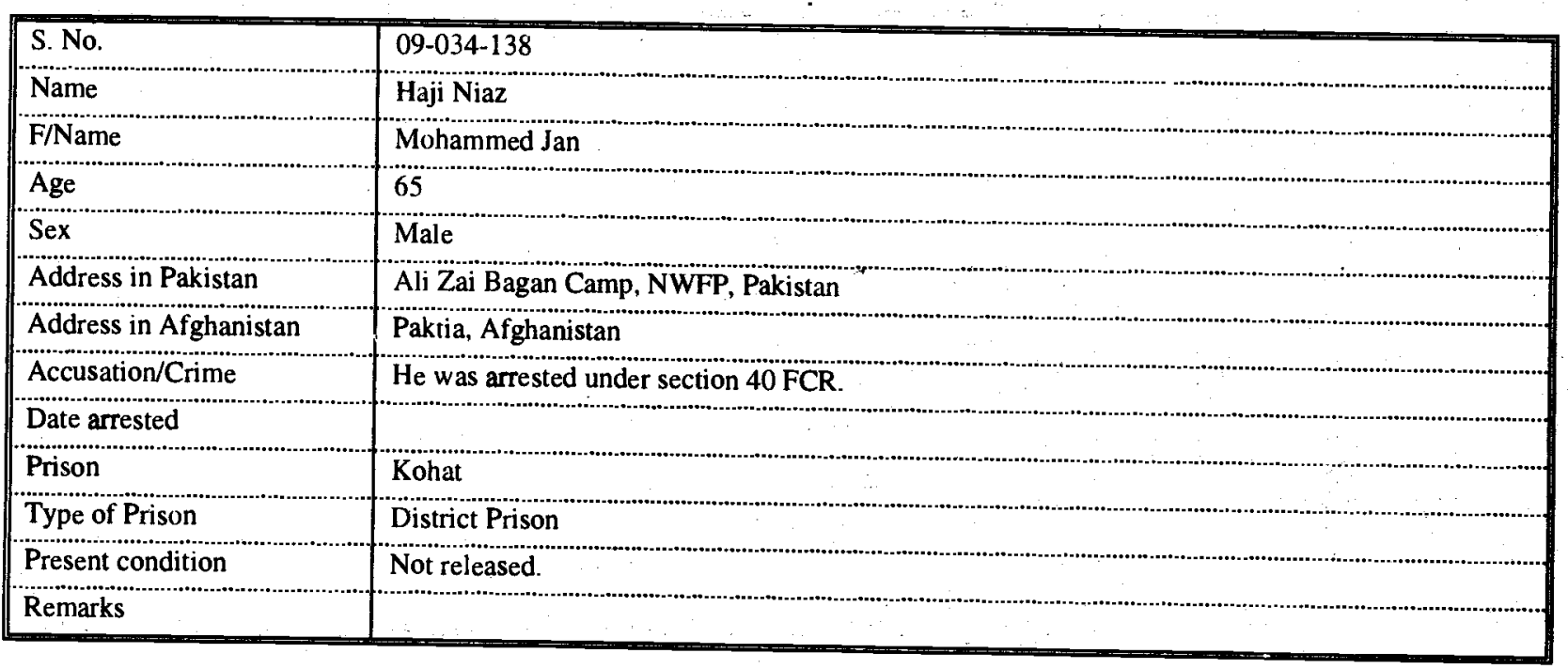




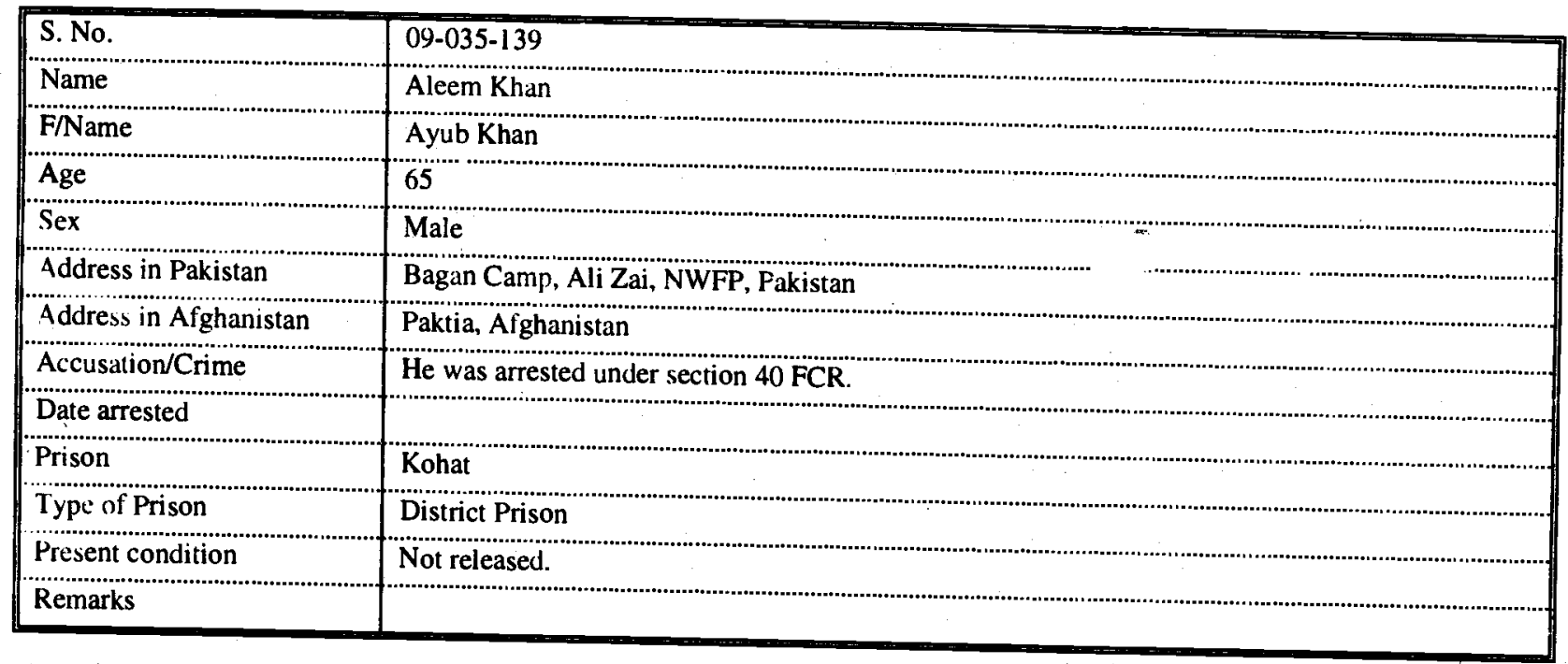

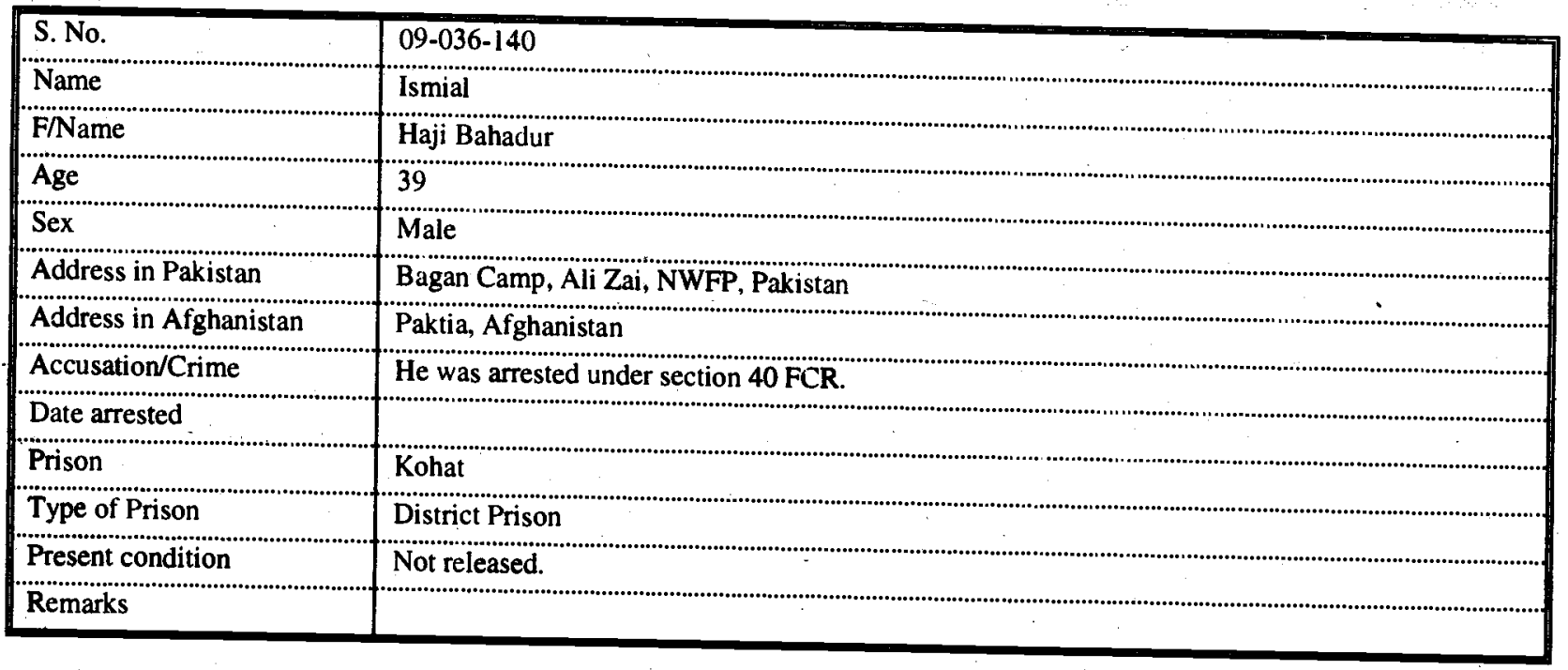

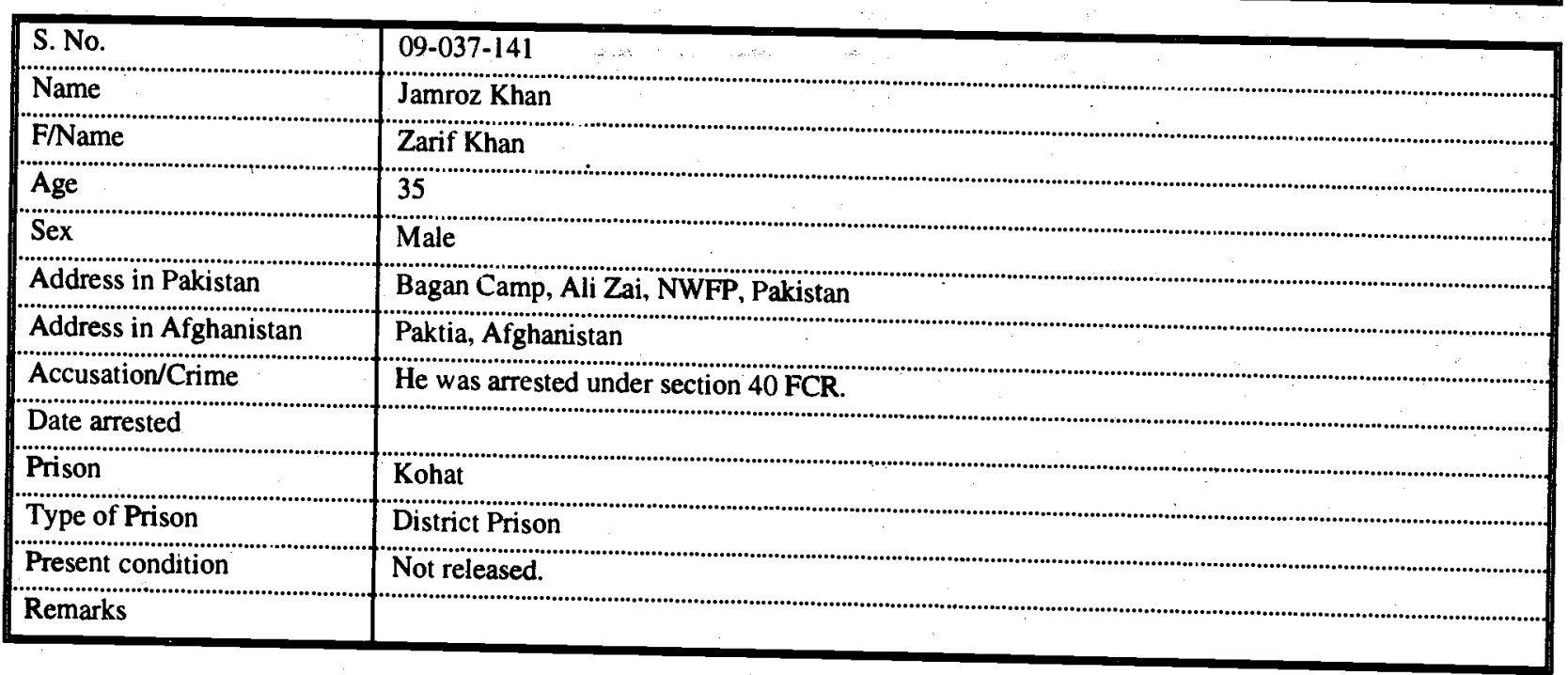




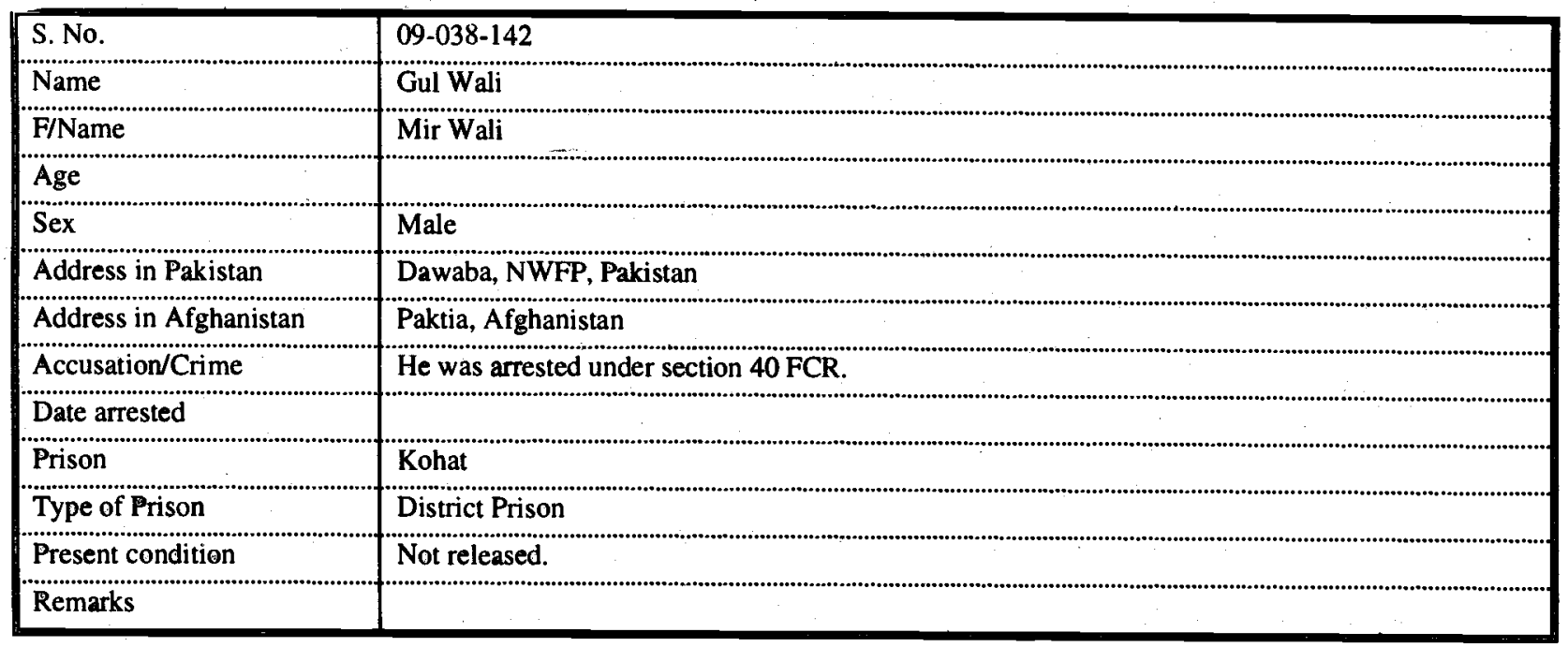

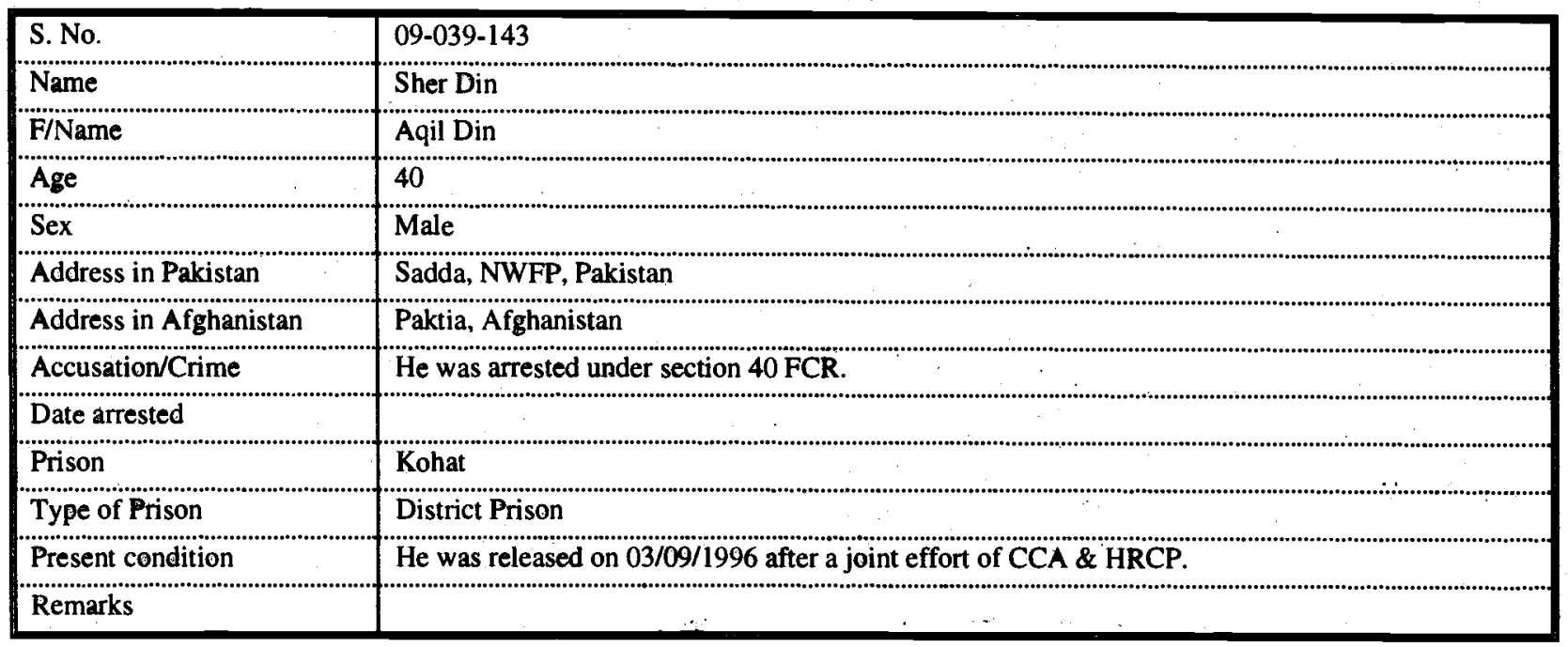

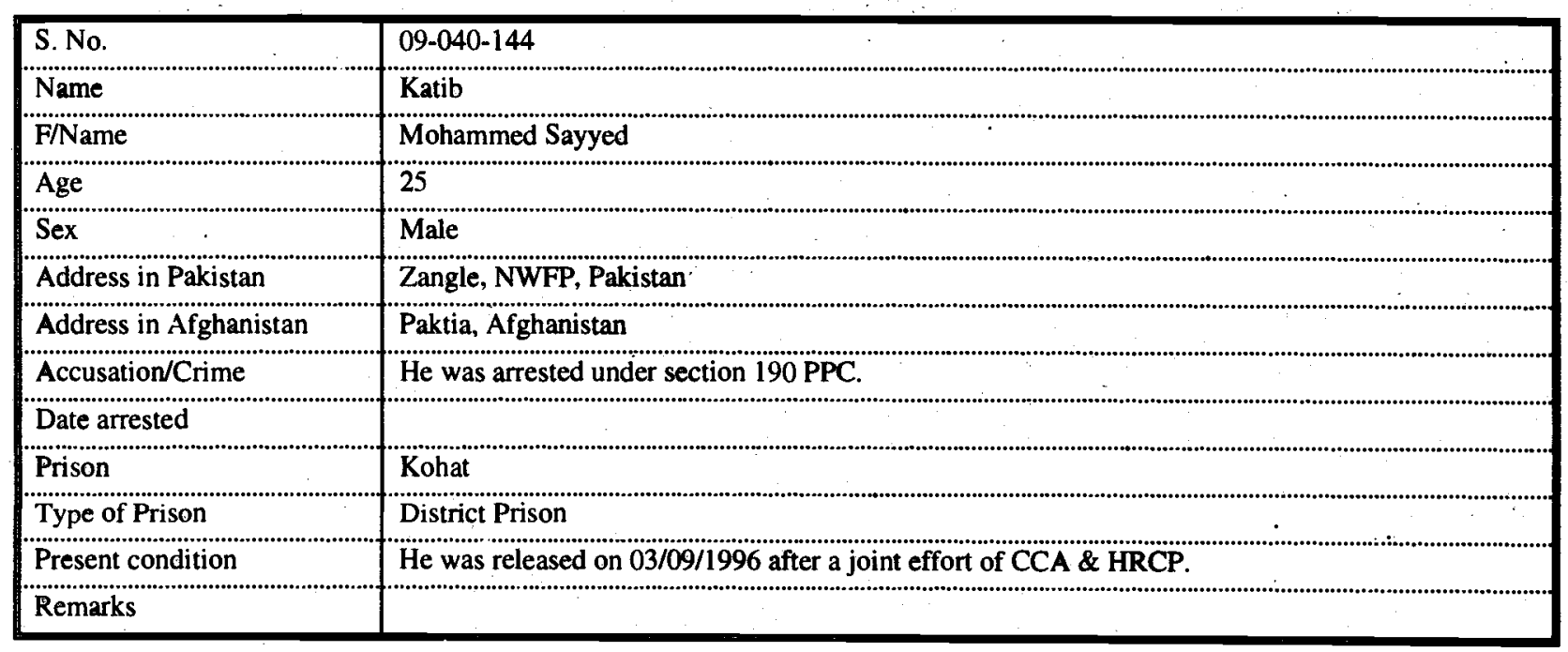




\begin{tabular}{|c|c|}
\hline S. No. & 09-041-145 \\
\hline Name & Shad Mehammed \\
\hline F/Name & Bari \\
\hline Age & 35 \\
\hline Sex & Male \\
\hline Address in Pakistan & Obland Camp, NWFP, Pakistan \\
\hline \multicolumn{2}{|c|}{ Address in Afghanistan } \\
\hline Accusation/Crime & He was arrested under section 109 PPC. \\
\hline \multicolumn{2}{|l|}{ Date arrested } \\
\hline Prison & Kohat \\
\hline Type of Prison & District Prison \\
\hline Present condition & He was released on $03 / 09 / 1996$ after a joint effort of CCA \& HRCP. \\
\hline Remarks & \\
\hline
\end{tabular}

\begin{tabular}{|c|c|}
\hline S. No. & $09-042-146$ \\
\hline Name & Sultan Khel \\
\hline F/Name & Nazar Khel \\
\hline Age & 40 \\
\hline Sex & Male \\
\hline Address in Pakistan & Kohat No. 1, NWFP, Pakistan \\
\hline \multicolumn{2}{|c|}{ Address in Afghanistan } \\
\hline Accusation/Crime & He was arrested under section 109. \\
\hline \multicolumn{2}{|l|}{ Date arrested } \\
\hline Prison & Kohat \\
\hline Type of Prison & District Prison \\
\hline Present condition & He was released on 03/09/1996 after a joint effort of CCA \& HRCP. \\
\hline Remarks & \\
\hline
\end{tabular}

\begin{tabular}{|c|c|}
\hline S. No. & $09-043-147$ \\
\hline Name & Jana Gul \\
\hline F/Name & Redi Gul \\
\hline Age & 18 \\
\hline Sex & Male \\
\hline Address in Pakistan & Mianwali, NWFP, Pakistan \\
\hline Address in Afghanistan & (1) \\
\hline Accusation/Crime & He was arrested under section 109 PPC. \\
\hline Date arrested & \\
\hline Prison & Kohat \\
\hline Type of Prison & District Prison \\
\hline Present condition & He was released on 03/09/1996 after a joint effort of CCA \& HRCP. \\
\hline Remarks & \\
\hline
\end{tabular}




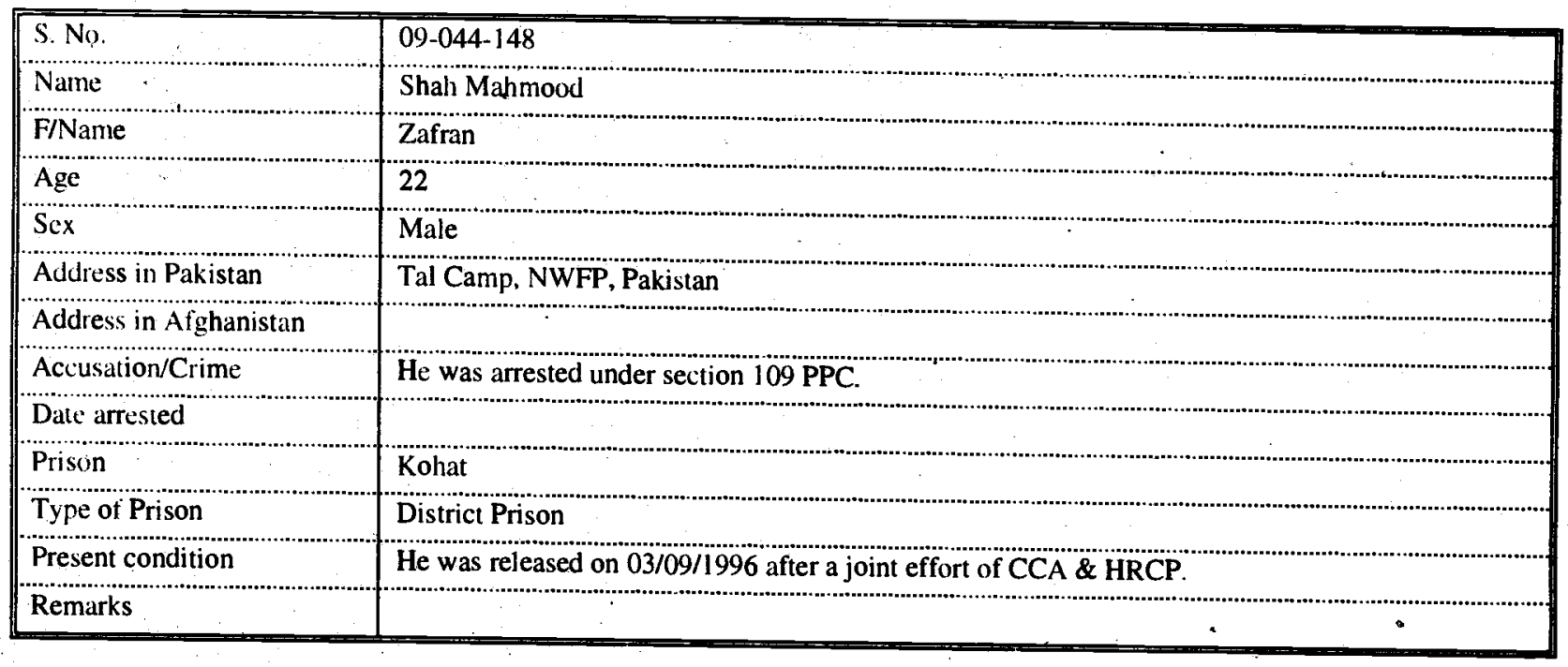

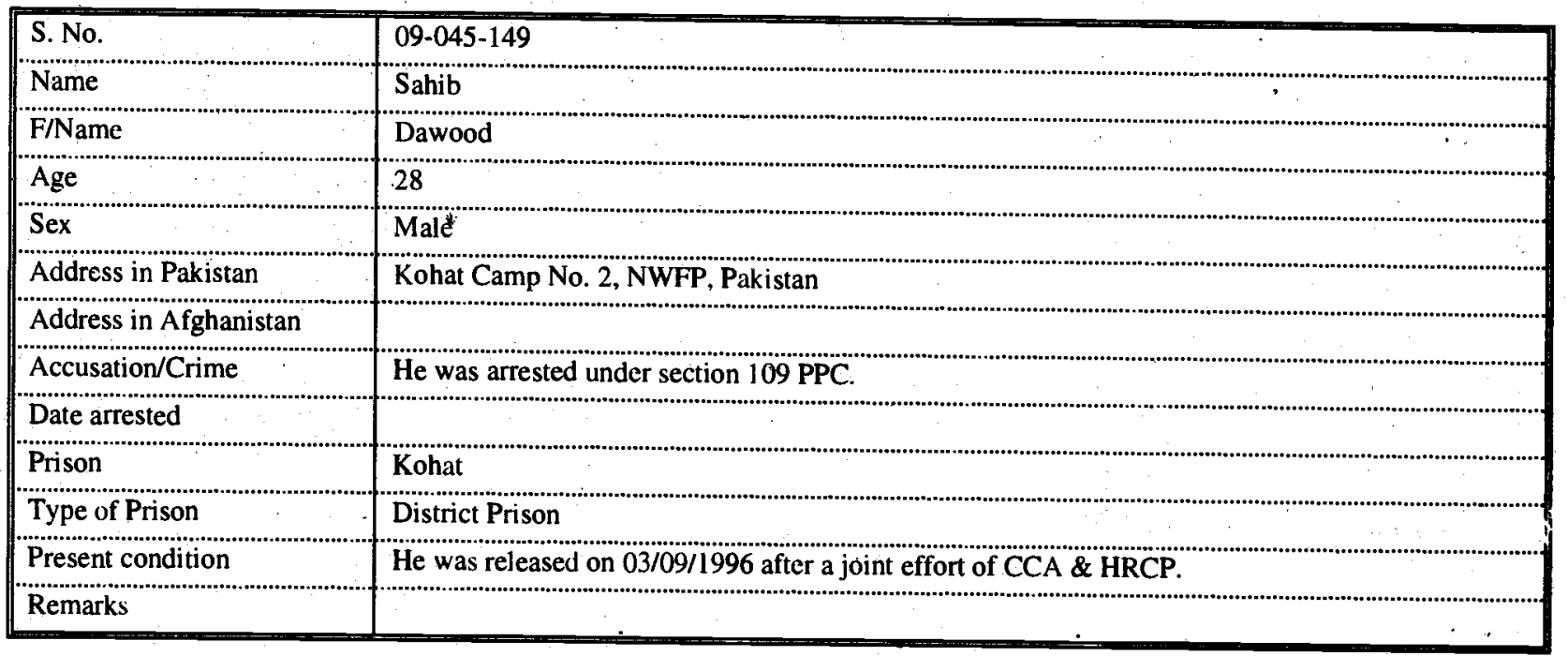

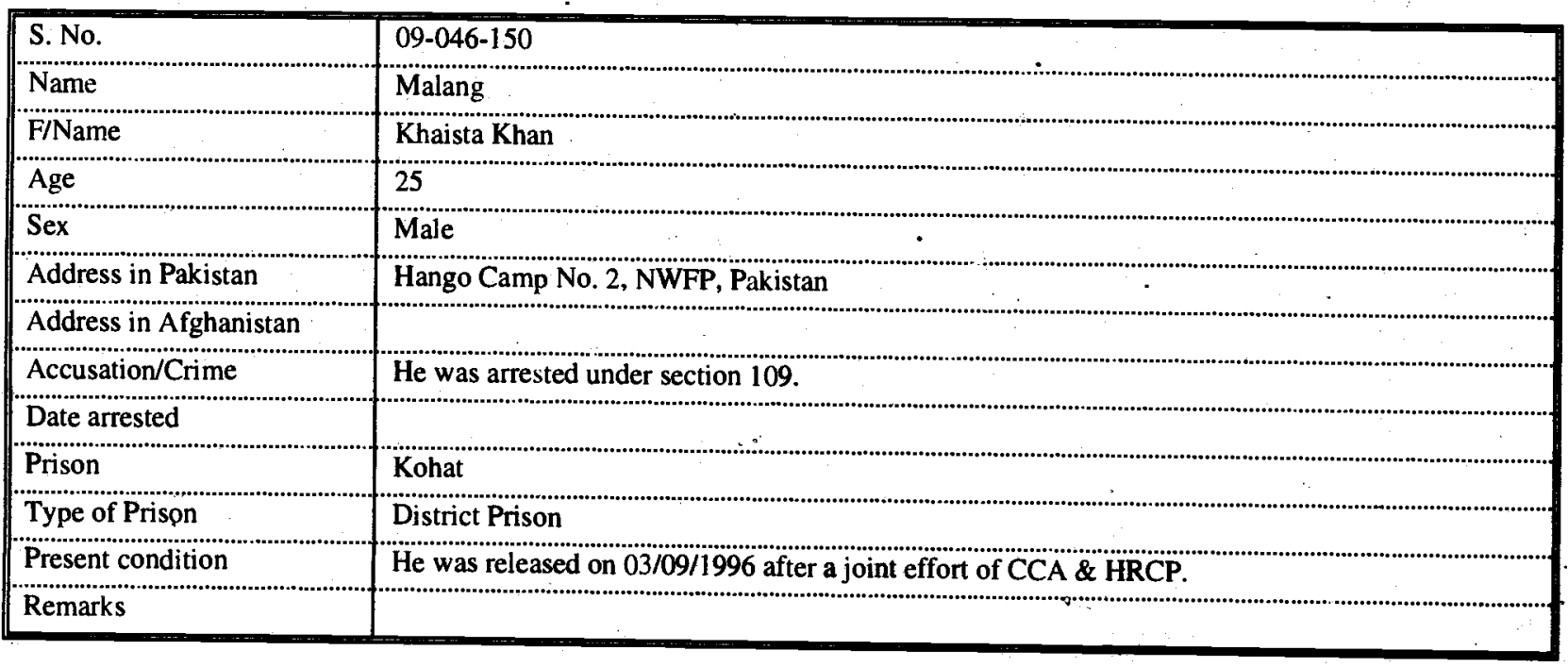




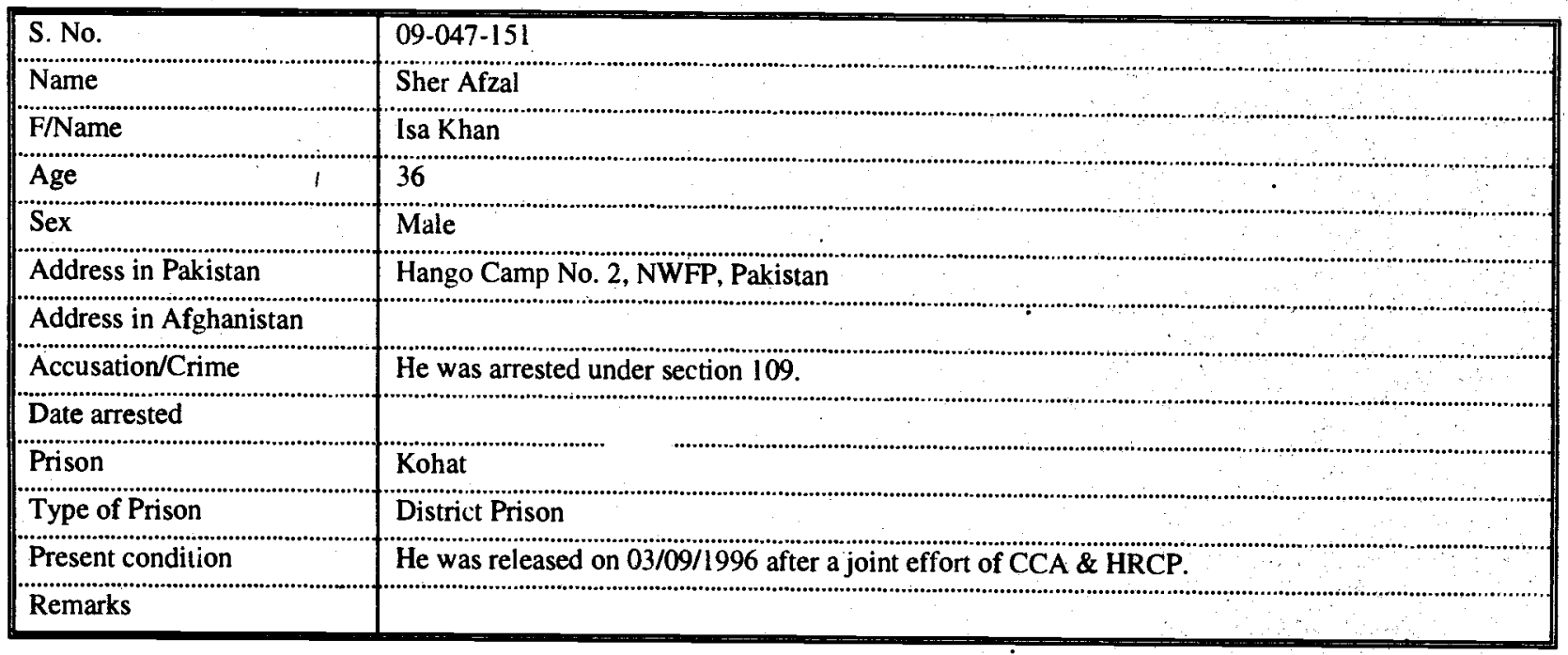

\begin{tabular}{|c|c|}
\hline S. No. & $09-048-152$ \\
\hline Name & Shamak \\
\hline F/Name & 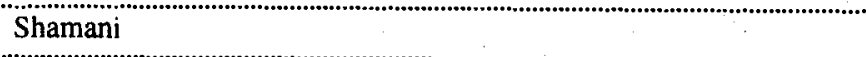 \\
\hline Age & 31 \\
\hline Sex & Male \\
\hline Accusation/Crime & He was arrested under section 109 PPC. \\
\hline Date arrested & \\
\hline Prison & Kohat \\
\hline Type of Prison & District Prison \\
\hline
\end{tabular}

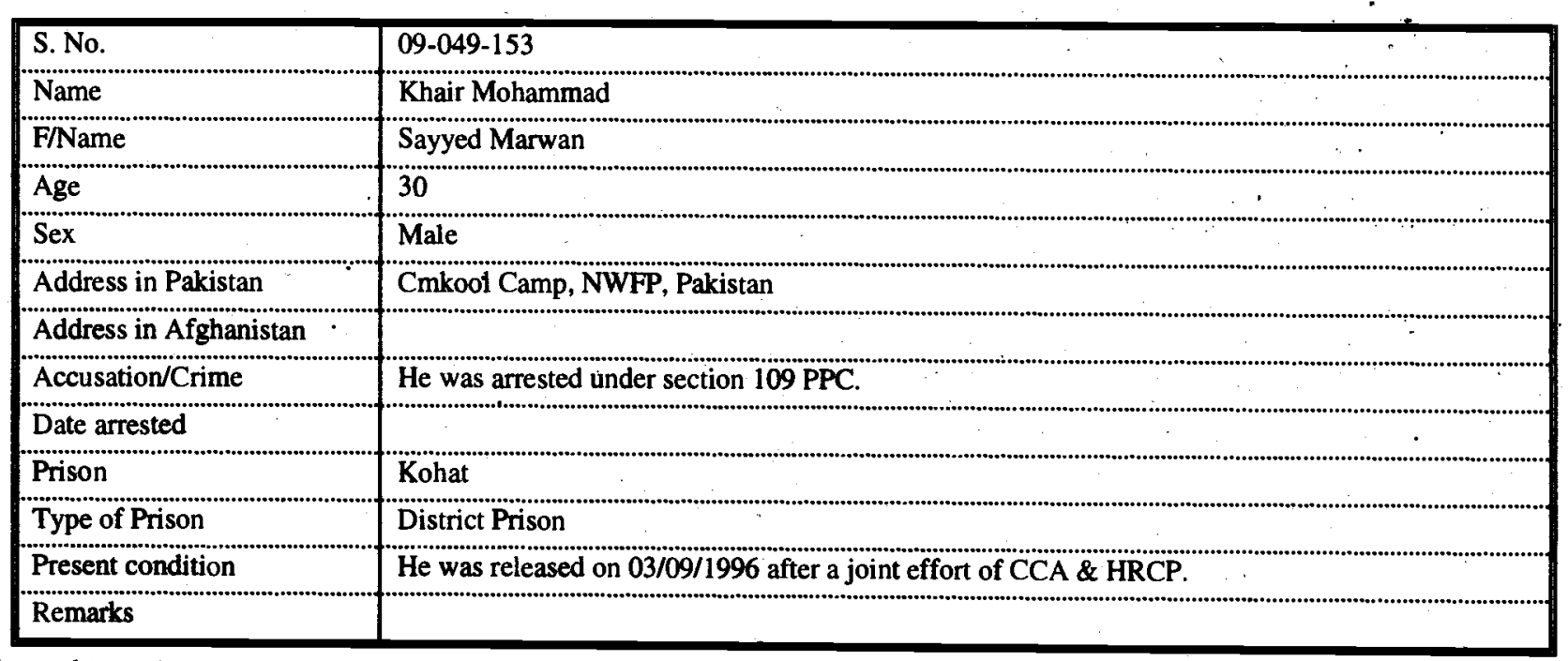




\begin{tabular}{|c|c|}
\hline S. No. & $09-050-154$ \\
\hline Name & Lal Marjan \\
\hline FiName & Qalander \\
\hline Age & 25 \\
\hline $\operatorname{Sex} \cdots$ & Male \\
\hline Address in Pakistan & Kohat Camp No. 1, NWFP, Pakistan \\
\hline \multicolumn{2}{|c|}{ Address in Afghanistan } \\
\hline Accusation/Crime & He was arrested under section 109 PPC. \\
\hline \multicolumn{2}{|l|}{ Date arrested } \\
\hline Prison & Kohat \\
\hline Type of Prison & District Prison \\
\hline Present condition & He was released on $03 / 09 / 1996$ after a joint effort of CCA \& HRCP. \\
\hline Remarks & \\
\hline
\end{tabular}

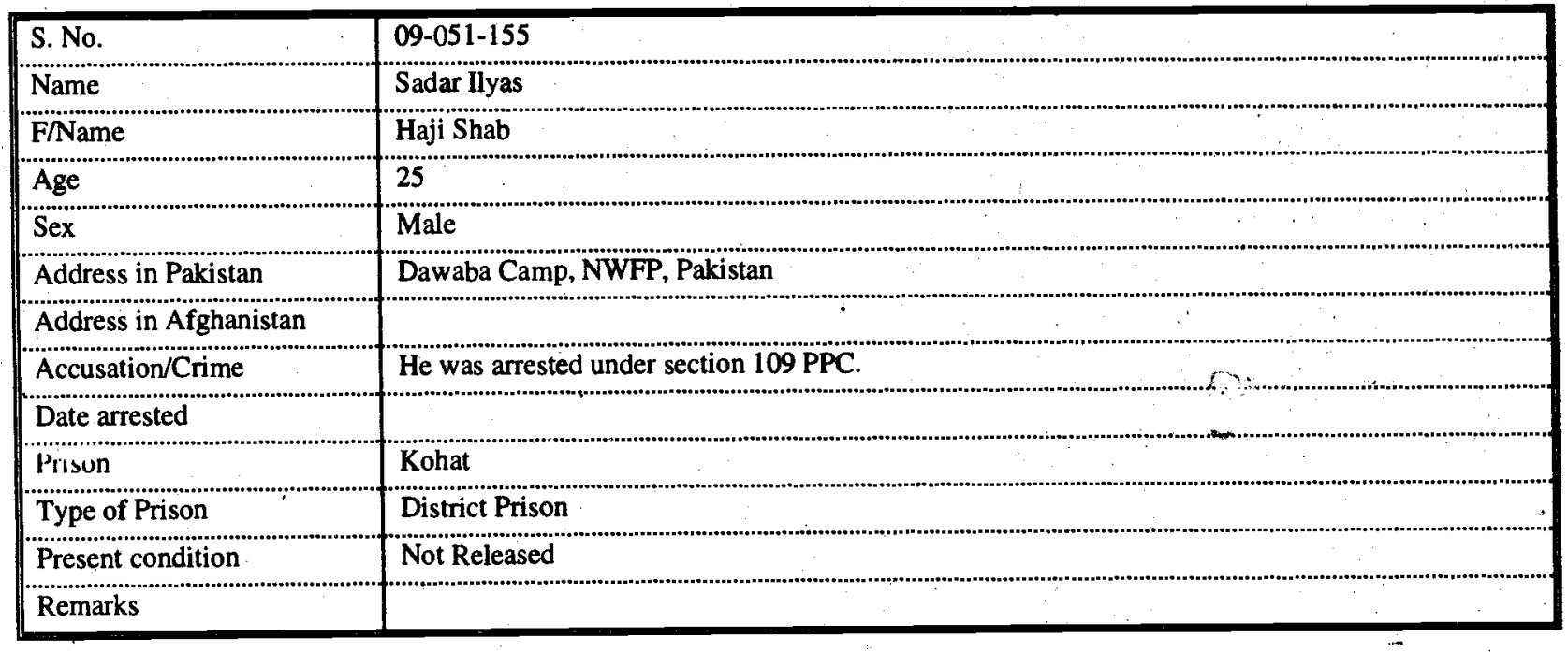

\begin{tabular}{|c|c|}
\hline S. No. & $09-052-156$ \\
\hline Nâme & Mohammed Raiz \\
\hline F/Name & Atta Khan \\
\hline Age & 34 \\
\hline Sex & Male \\
\hline Address in Pakistan & Kohat Camp No. 2, NWFP, Pakistan \\
\hline \multicolumn{2}{|c|}{ Address in Afghanistan } \\
\hline Accusation/Crime & He was arrested under section 107 PPC. \\
\hline Date arrested & , \\
\hline Prison & Kohat \\
\hline Type of Prison & District Prison \\
\hline Present condition & Not released. \\
\hline Remarks & \\
\hline
\end{tabular}




\begin{tabular}{|c|c|}
\hline S. No. & $09-053-157$ \\
\hline Name & Shafiqullah \\
\hline F/Name & Salih Shah \\
\hline Age & 30 \\
\hline Sex & Male \\
\hline Address in Pakistan & Kohat Camp, NWFP, Pakistan \\
\hline Address in Afghanistan & \\
\hline Accusation/Crime & He was arrested under section 109 PPC. \\
\hline Date arrested & \\
\hline Prison & Kohat \\
\hline Type of Prison & District Prison \\
\hline Present condition & Not released \\
\hline Remarks & \\
\hline
\end{tabular}

\begin{tabular}{|c|c|}
\hline S. No. & $09-054-158$ \\
\hline Name & Khan Zaman \\
\hline F/Name & Salih Uddin \\
\hline Age & 35 \\
\hline Sex & Male \\
\hline Address in Pakistan & Kohat Camp, NWFP, Pakistan \\
\hline \multicolumn{2}{|c|}{ Address in Afghanistan } \\
\hline Accusation/Crime & He was arrested.under section 109 PPC. \\
\hline \multicolumn{2}{|l|}{ Date arrested } \\
\hline Prison & Kohat \\
\hline Type of Prison & District Prison \\
\hline Present condition & He was released on 03/09/1996 after a joint effort of CCA \& HRCP. \\
\hline Remarks & \\
\hline
\end{tabular}

\begin{tabular}{|c|c|}
\hline S. No. & $09-055-159$ \\
\hline Name & Munir Shah \\
\hline F/Name & Amir Khan \\
\hline Age & 35 \\
\hline Sex & Male \\
\hline Address in Pakistan & Kohat Camp, NWFP, Pakistan \\
\hline Address in Afghanist & \\
\hline Accusation/Crime & He was arrested under section $4 \mathrm{PO}$. \\
\hline Date arrested & \\
\hline Prison & Kohat \\
\hline Type of Prison & District Prison \\
\hline Present condition & \\
\hline Remarks & \\
\hline
\end{tabular}




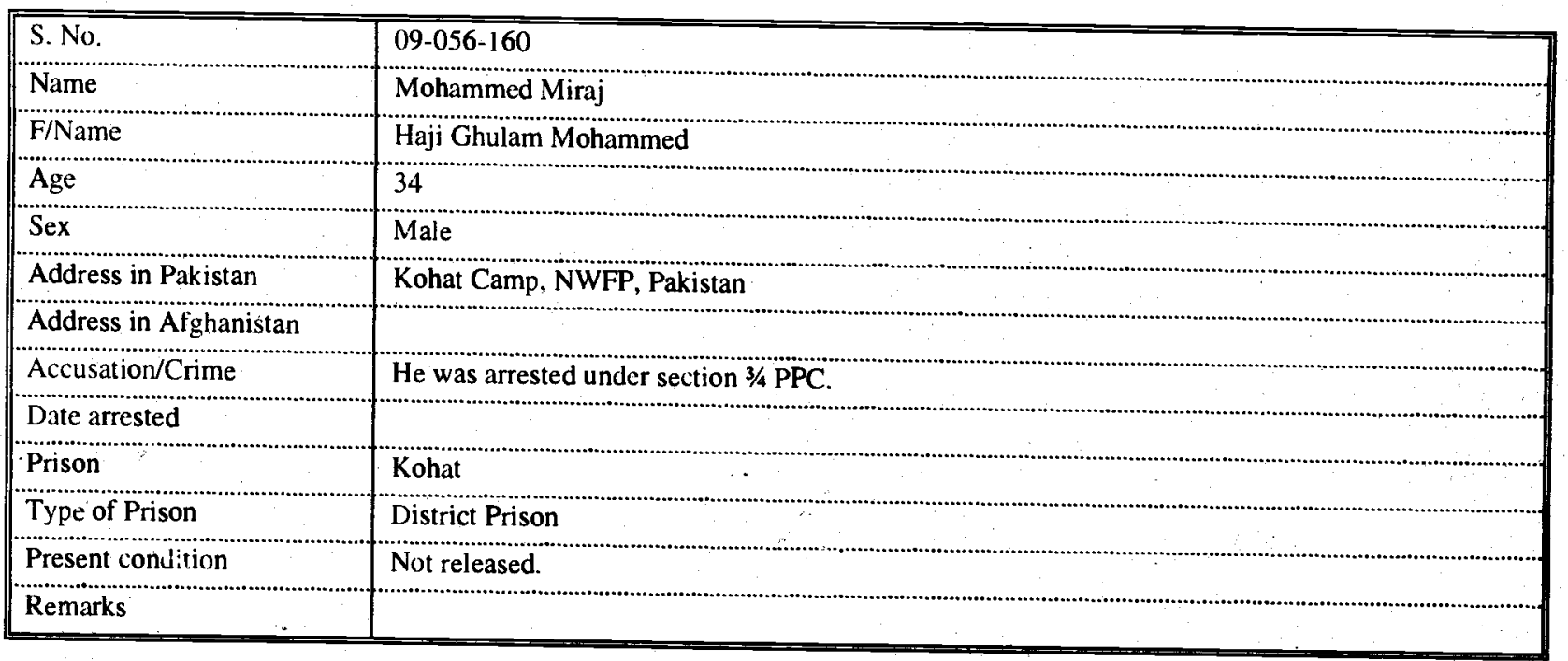

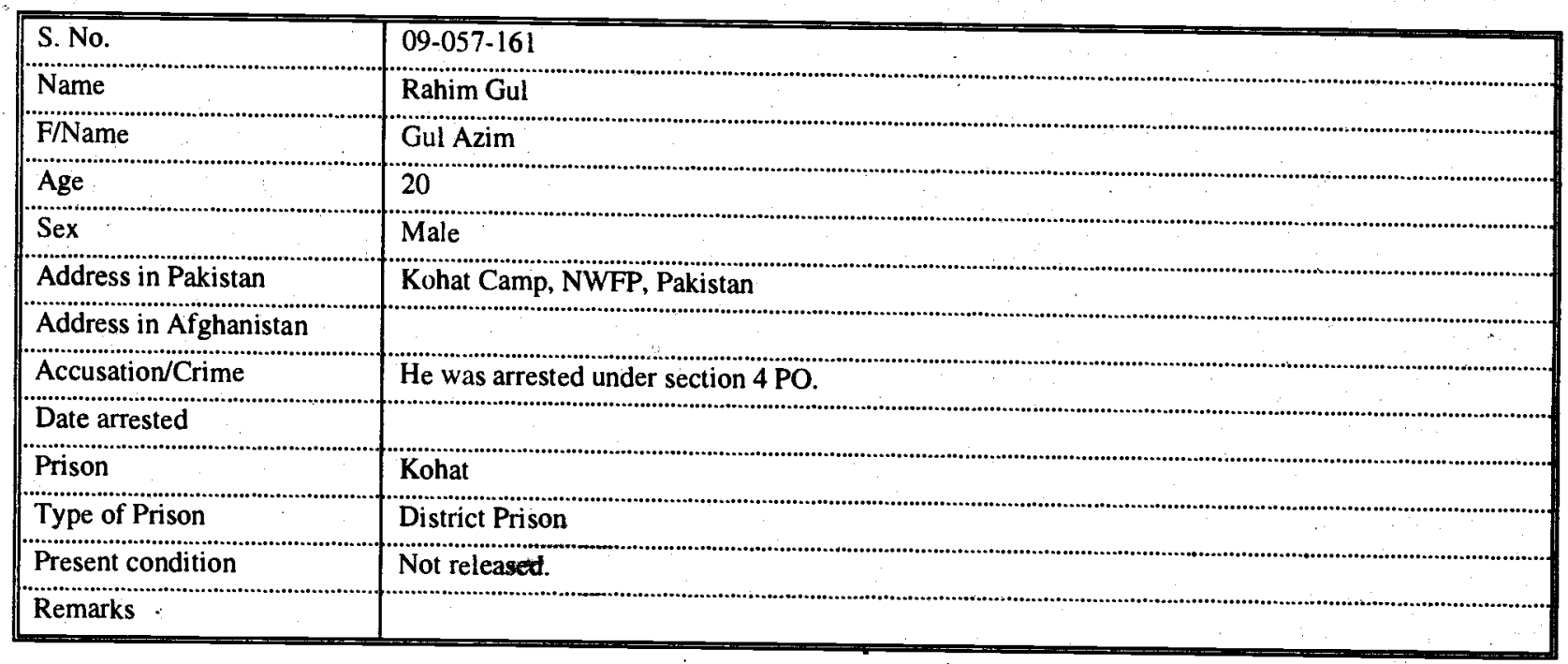

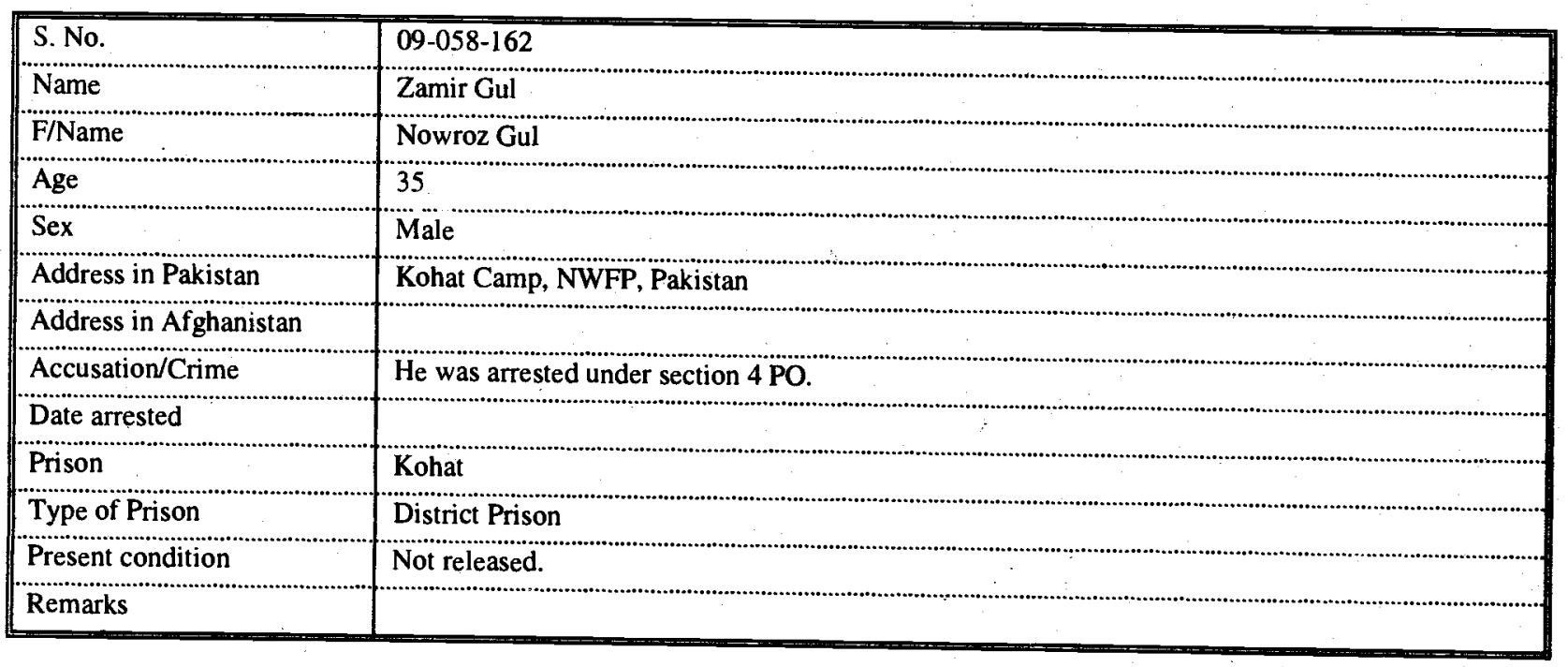




\begin{tabular}{|c|c|}
\hline S. No. & $09-059-163$ \\
\hline Name & Iqbal \\
\hline F/Name & Habibullah \\
\hline Age & 31 \\
\hline Sex & Male \\
\hline Address in Pakistan & Kohat Camp, NWFP, Pakistan \\
\hline Accusation/Crime & He was arrested under section $3 / 4$ PPC. \\
\hline \multicolumn{2}{|l|}{ Date arrested } \\
\hline Prison. & Kohat \\
\hline Type of Prison & District Prison \\
\hline
\end{tabular}

\begin{tabular}{|c|c|}
\hline S. No. & 09-060-164 \\
\hline Name & Khaliq Mir \\
\hline F/Name & Mir Jamal \\
\hline Age & 38 \\
\hline Sex & Male \\
\hline Accusation/Crime & He was arrested under section $4 \mathrm{PO}$. \\
\hline Date arrested & \\
\hline Prison. & Kohat \\
\hline Type of Prison & District Prison \\
\hline
\end{tabular}

\begin{tabular}{|c|c|}
\hline S. No. & $09-061-165$ \\
\hline F/Name & Mohammad Khalid . \\
\hline Age & 25 \\
\hline Sex & Male \\
\hline Accusation/Crime & He was arrested under section 107 PPC. \\
\hline Date arrested & \\
\hline Prison & Kohat \\
\hline
\end{tabular}




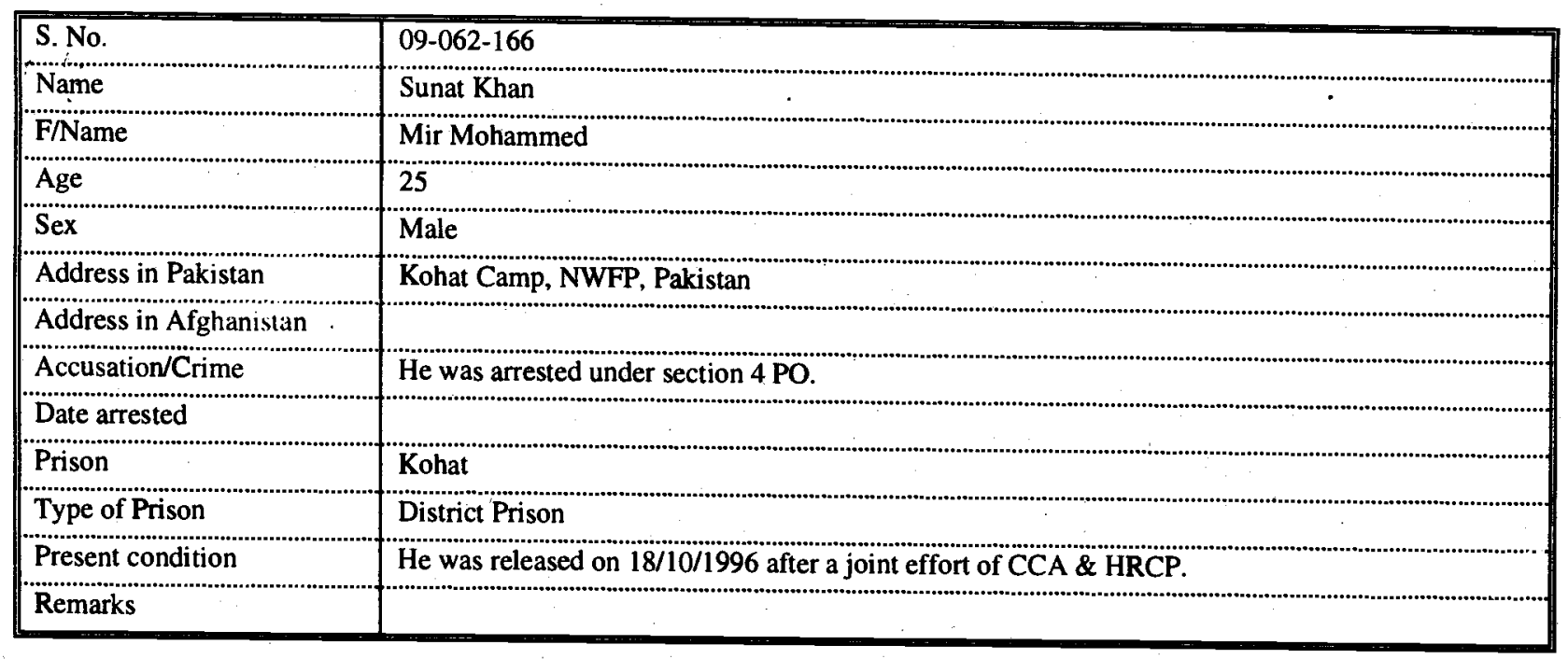

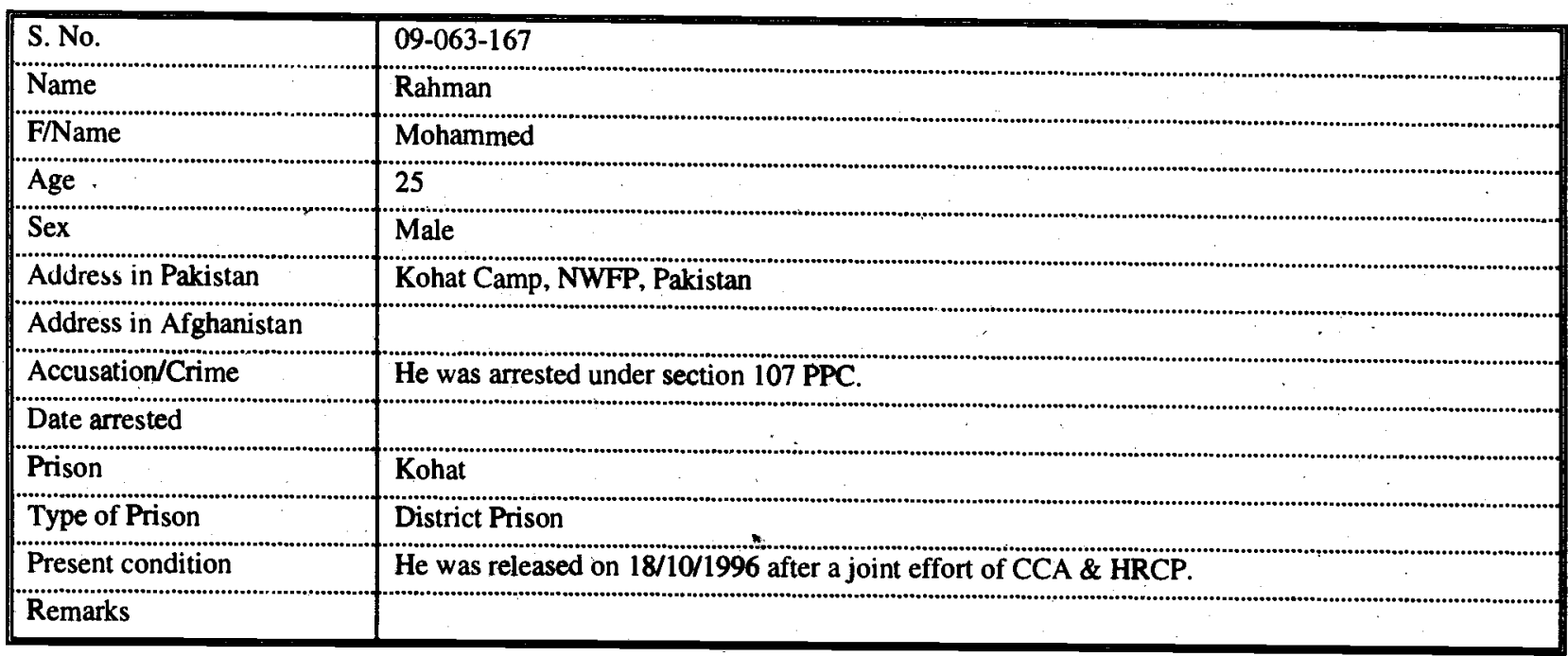

\begin{tabular}{|c|c|}
\hline S. No. & $09-064-168$ \\
\hline Name & Mubarak Ali \\
\hline F/Name & Mohammed Janan \\
\hline Age & 25 \\
\hline Sex & Male \\
\hline Address in Pakistan & Kohat Camp, NWFP, Pakistan \\
\hline Address in Afghanistan & 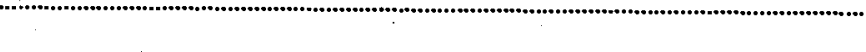 \\
\hline Accusation/Crime & He was arrested under section $3 / 4$ \\
\hline Date arrested & $x^{\prime}$ \\
\hline Prison & Kohat \\
\hline Type of Prison & District Prison \\
\hline Present condition & He was released on $18 / 10 / 1996$ after a joint effort of CCA \& HRCP. \\
\hline Remarks & (1) \\
\hline
\end{tabular}




\begin{tabular}{|c|c|}
\hline S. No. & 09-065-169 \\
\hline Name & Arab Khan \\
\hline F/Name & Akabar Khan \\
\hline Age & 25 \\
\hline Sex & Male \\
\hline Address in Pakistan & Kohat Camp, NWFP, Pakistan \\
\hline \multicolumn{2}{|c|}{ Address in Afghanistan } \\
\hline Accusation/Crime & He was arrested under section 107 PPC. \\
\hline \multicolumn{2}{|l|}{ Date arrested } \\
\hline Prison & Kohat \\
\hline Type of Prison & District Prison \\
\hline Present condition & He was released on 18/10/1996 after a joint effort of CCA \& HRCP. \\
\hline Remarks & 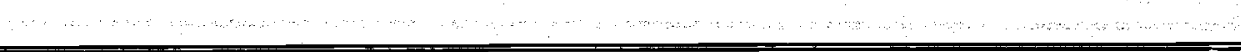 \\
\hline
\end{tabular}

\begin{tabular}{|c|c|}
\hline S. No. & 09-066-170 \\
\hline Name & Mohammed Afzal \\
\hline F/Name & Noor Hussain \\
\hline Age & 29 \\
\hline Sex & Male \\
\hline Address in Pakistan & Kohat Camp, NWFP, Pakistan \\
\hline Address in Afghanistan & \\
\hline Accusation/Crime & He was arrested under section 107 PPC. \\
\hline Date arrested & \\
\hline Prison & Kohat \\
\hline Type of Prison & District Prison \\
\hline Present condition & He was released on $18 / 10 / 1996$ after a joint effort of CCA \& HRCP. \\
\hline 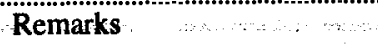 & 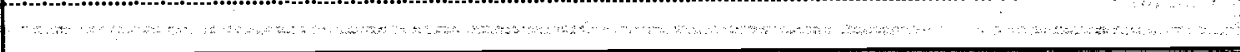 \\
\hline
\end{tabular}

\begin{tabular}{|c|c|}
\hline S. No. & $09-067-171$ \\
\hline Name & Mujahidin \\
\hline F/Name & (n) \\
\hline Age. & 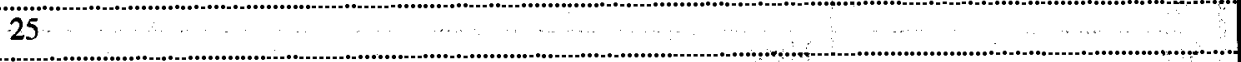 \\
\hline Sex & …............. \\
\hline Address in Pakistan & Kohat Camp No. 2, NWFP, Pakistan \\
\hline Address in Afghanistan & (-) \\
\hline Accusation/Crime & He was arrested under section 107 PPC. \\
\hline \multicolumn{2}{|l|}{ Date arrested } \\
\hline Prison & Kohat \\
\hline Type of Prison & District Prison \\
\hline Present condition & He was released on $18 / 10 / 1996$ after a joint effort of CCA \& HRCP. \\
\hline Remarks & (n) \\
\hline
\end{tabular}




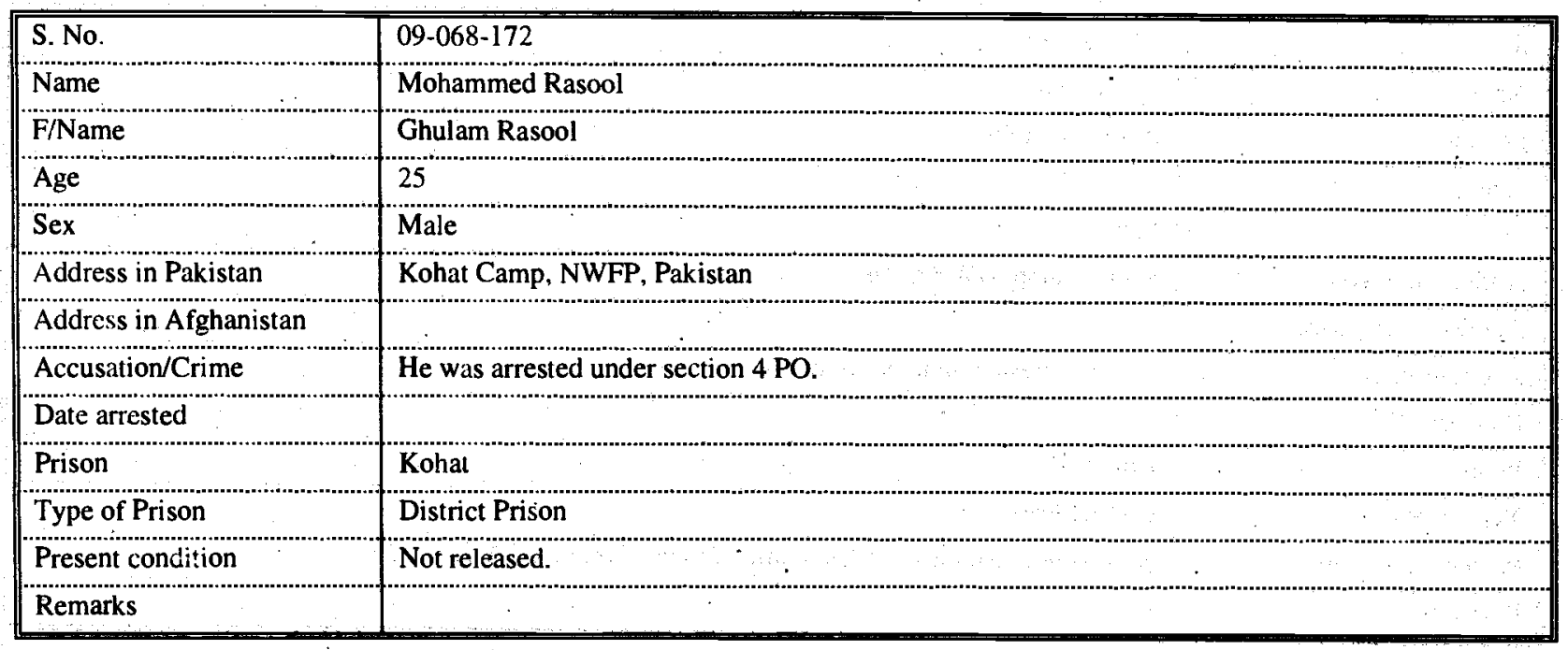

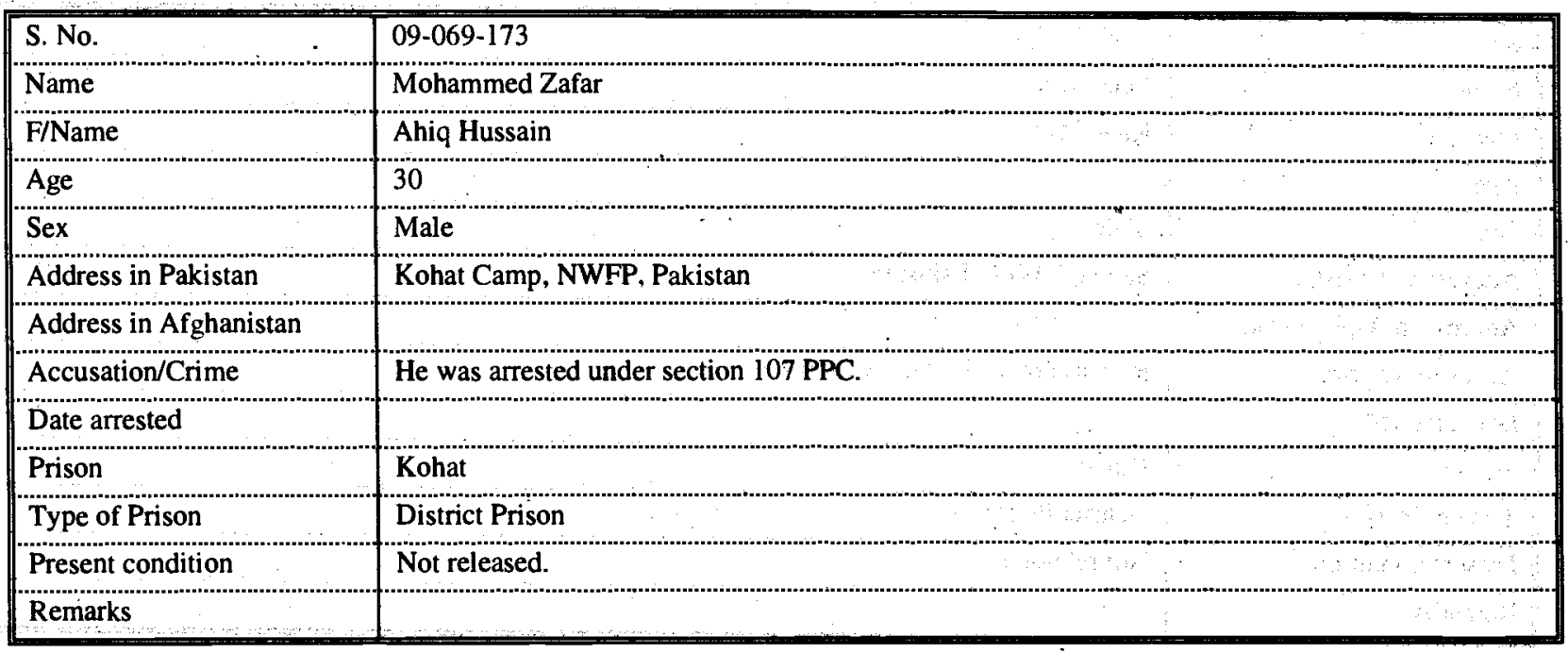

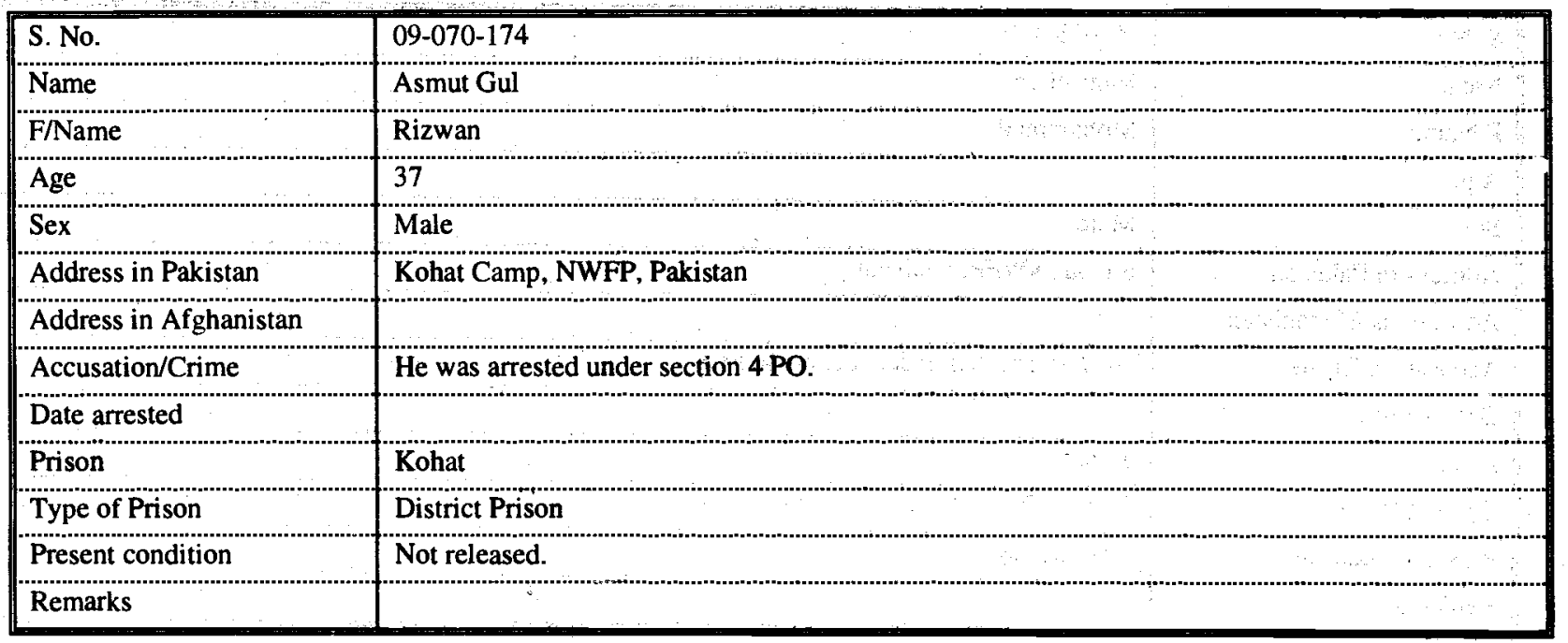




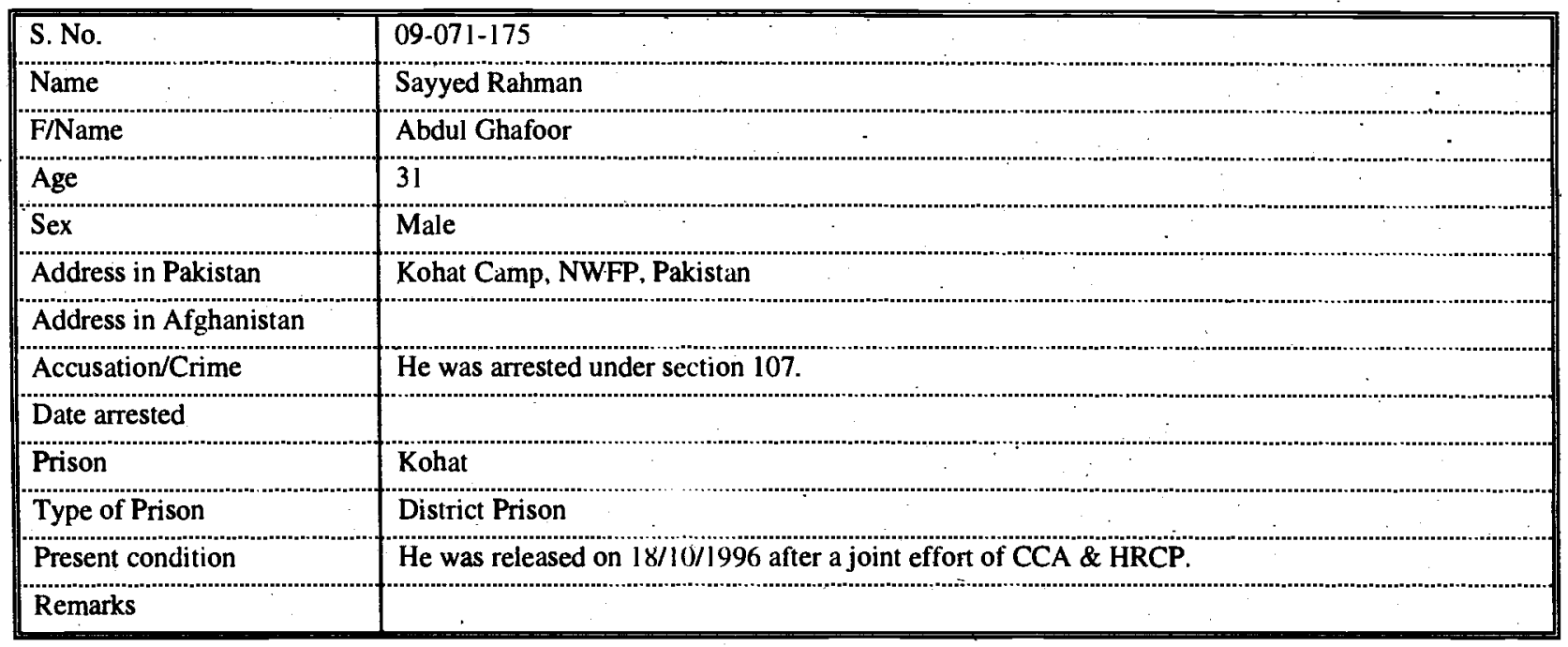

\begin{tabular}{|l|l||}
\hline S. No. & $09-072-176$ \\
\hline Name & Janat Mir \\
\hline F/Name & Khan Mir \\
\hline Age & Male \\
\hline Sex & Sadda, NWFP, Pakistan \\
\hline Address in Pakistan & \\
\hline Address in Afghanistan & He was arrested under section 40 FCR. \\
\hline Accusation/Crime & Kohat \\
\hline $\begin{array}{l}\text { Date arrested } \\
\text { Prison }\end{array}$ & District Prison \\
\hline Type of Prison & Not released. \\
\hline \begin{tabular}{l} 
Present condition \\
\hdashline Remarks
\end{tabular} & \\
\hline
\end{tabular}

\begin{tabular}{|l|l|}
\hline S. No. & $09-073-177$ \\
\hline Name & Hanifullah \\
\hline F/Name & Mohammed \\
\hline Age & Male \\
\hline Sex & Sadda, NWFP, Pakistan \\
\hline Address in Pakistan & \\
\hline Address in Afghanistan & He was arrested under section 40 FCR. \\
\hline Accusation/Crime & \\
\hline \begin{tabular}{l} 
Date arrested \\
\hdashline Krison
\end{tabular} & Dohat \\
\hline $\begin{array}{l}\text { Type of Prison } \\
\text { Present condition }\end{array}$ & Not released. \\
\hline Remarks & \\
\hline
\end{tabular}




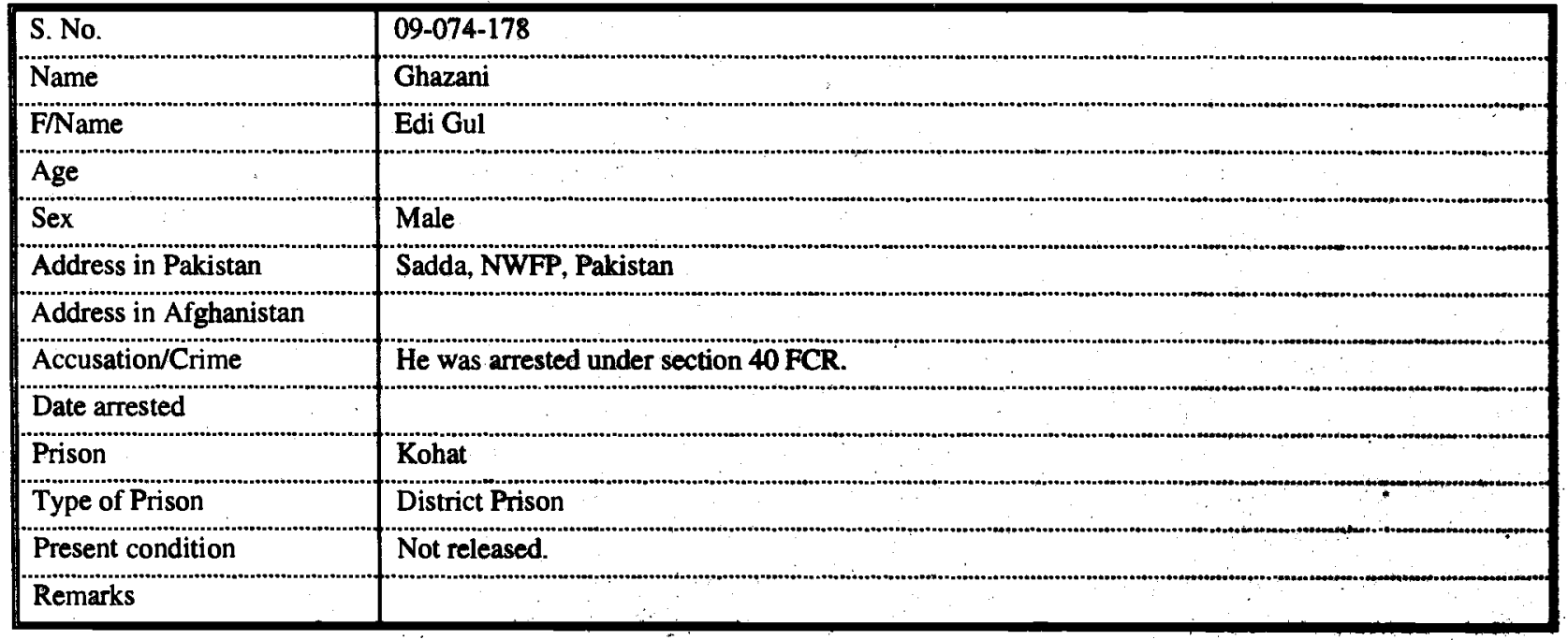

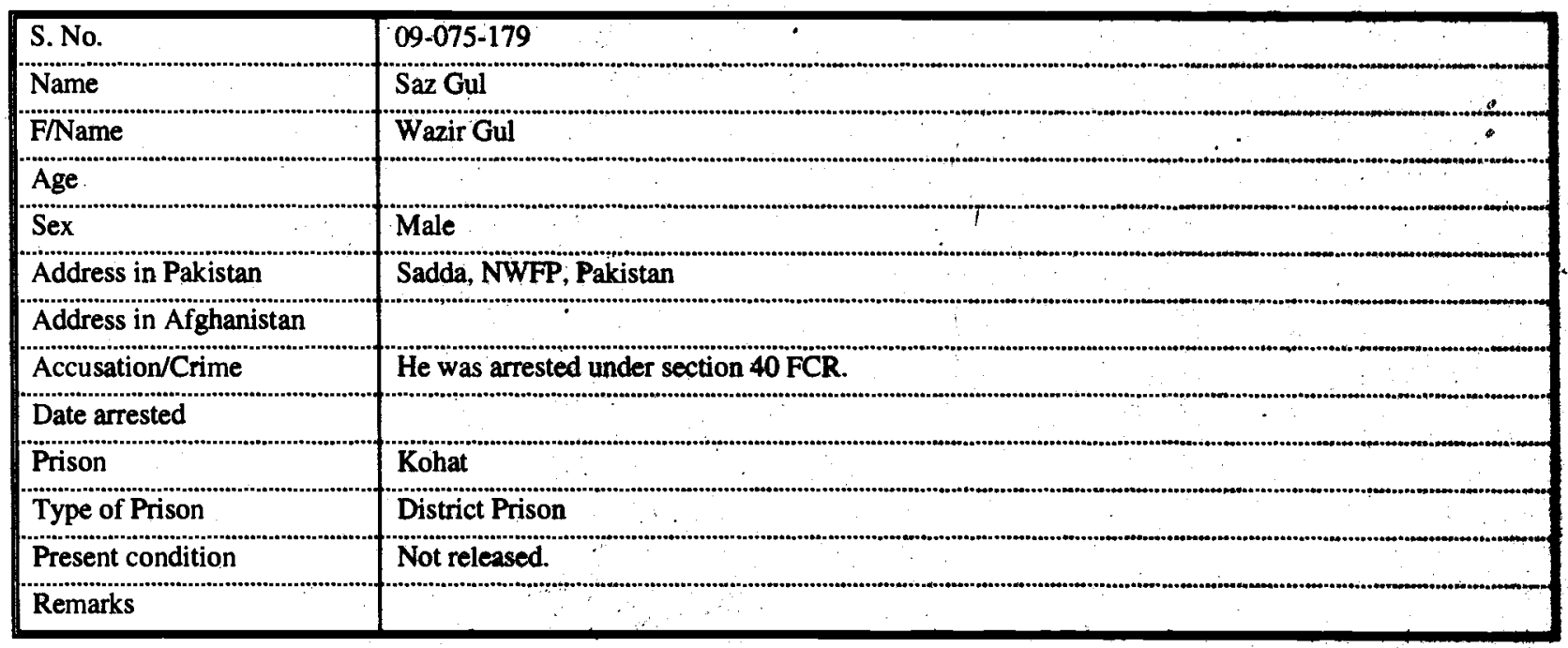

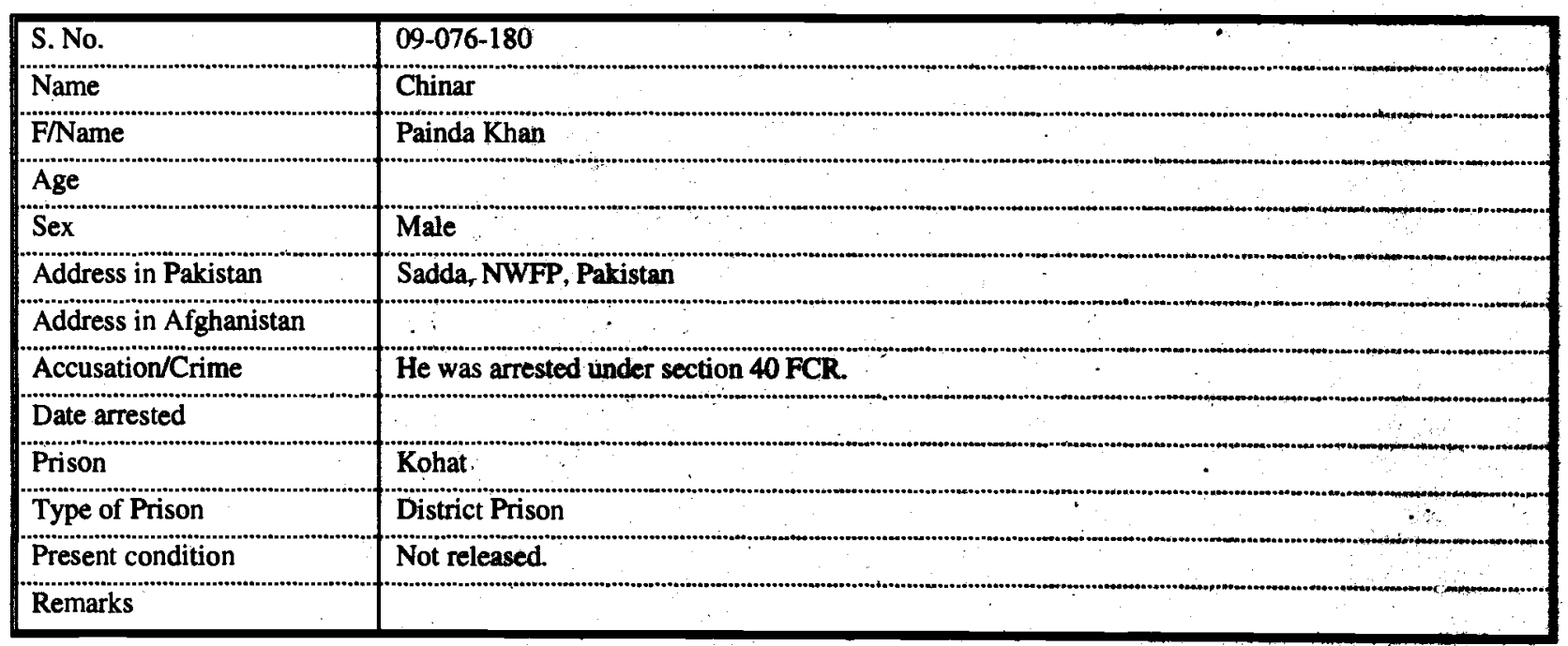




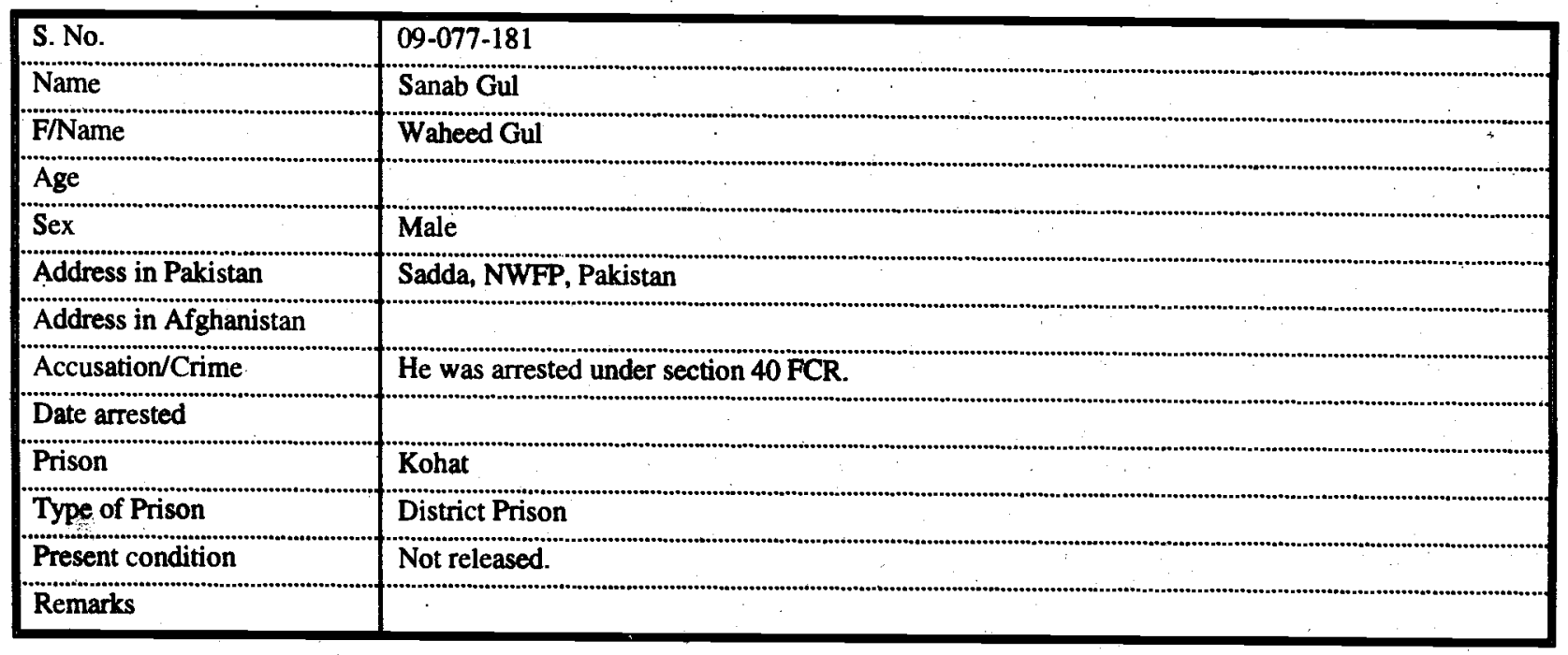

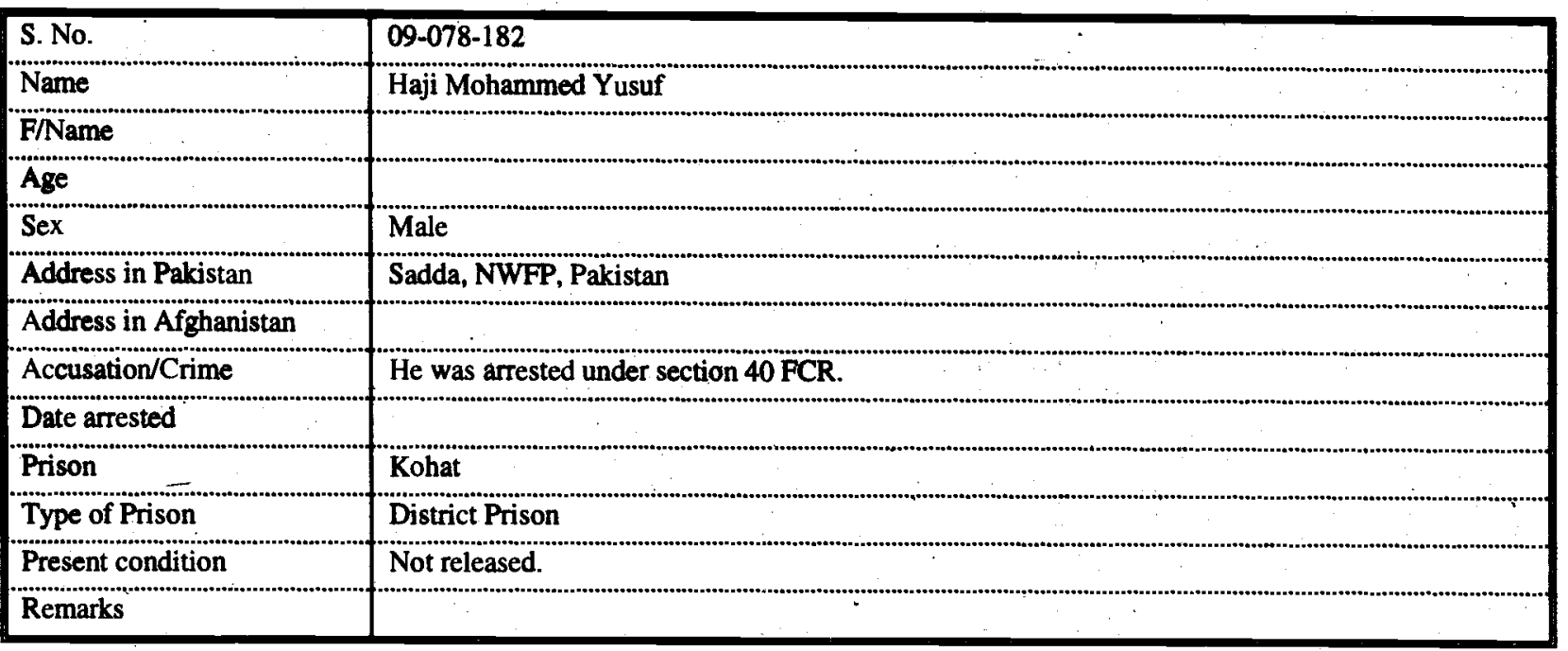

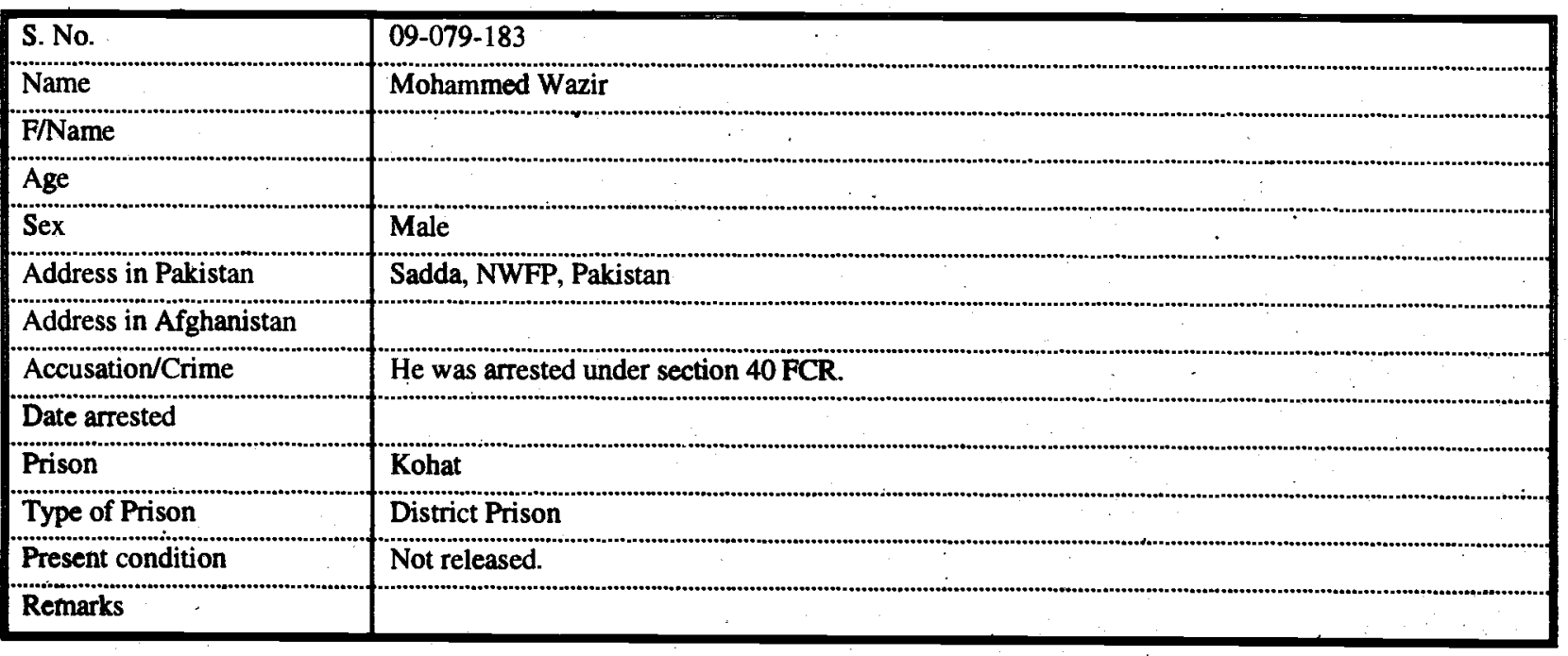




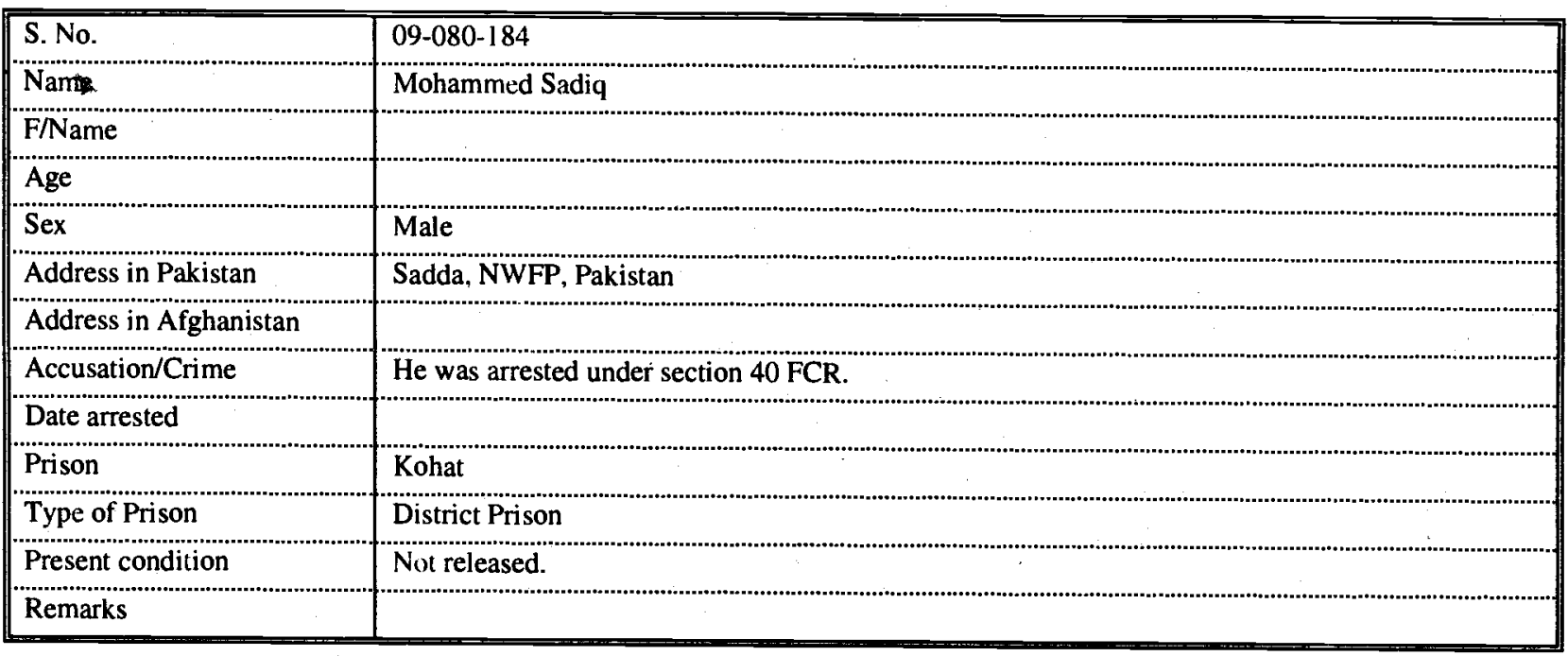

\begin{tabular}{|c|c|}
\hline S. No. & $09-081-185$ \\
\hline F/Name & \\
\hline Age & \\
\hline Sex & Male \\
\hline Accusation/Crime & He was arrested under section 40 FCR. \\
\hline Date arrested & \\
\hline Prison & Kohat \\
\hline Type of Prison & District Prison \\
\hline
\end{tabular}

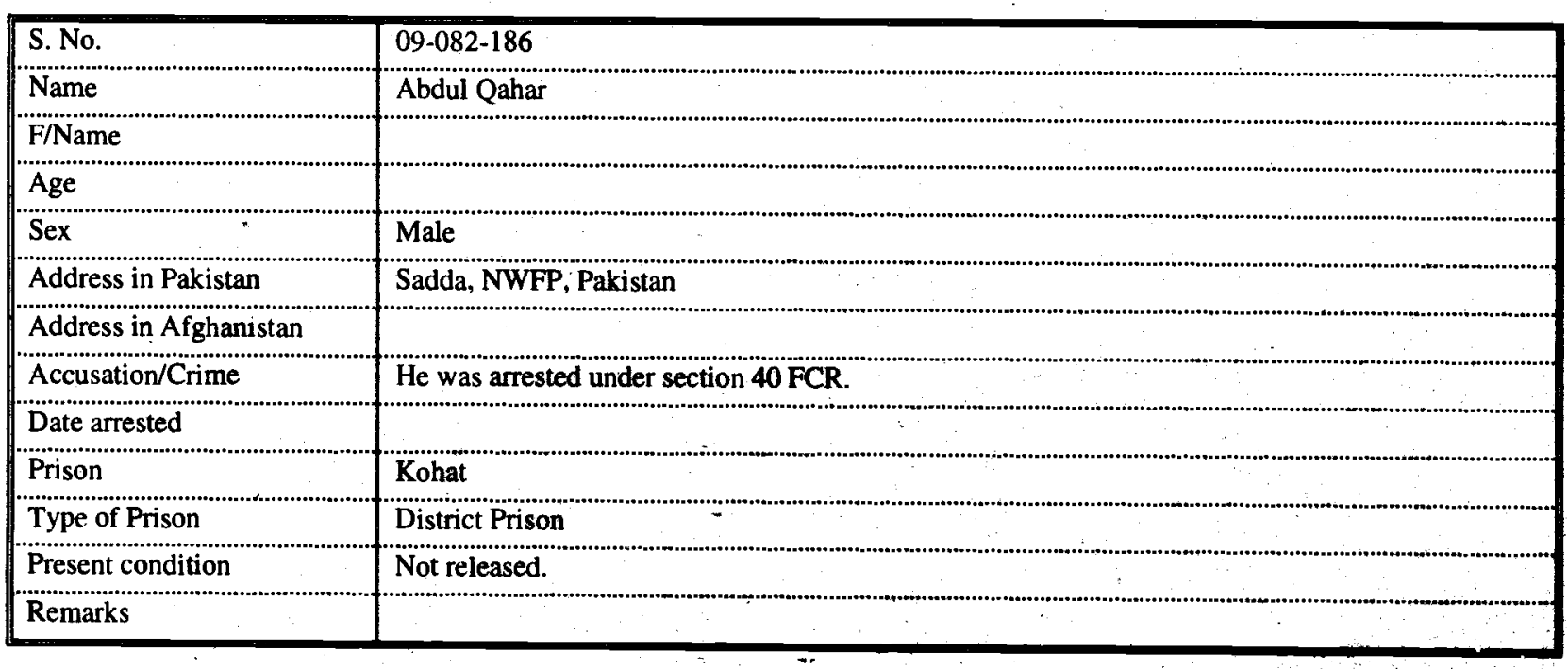




\begin{tabular}{|c|c|}
\hline S. No. & $09-083-187$ \\
\hline Name & Naweed Gul \\
\hline \multicolumn{2}{|l|}{ F/Name } \\
\hline \multicolumn{2}{|l|}{ Age } \\
\hline Sex & Male \\
\hline Address in Pakistan & Sadda, NWFP, Pakistan \\
\hline \multicolumn{2}{|c|}{ Address in Afghanistan } \\
\hline Accusation/Crime & He was arrested under section $40 \mathrm{FCR}$. \\
\hline \multicolumn{2}{|l|}{ Date arrested } \\
\hline Prison & Kohat \\
\hline Type of Prison & District Prison \\
\hline Present condition & Not released. \\
\hline Remarks & \\
\hline
\end{tabular}

\begin{tabular}{|c|c|}
\hline S. No. & $09-084-188$ \\
\hline Name & Momin Khan \\
\hline \multicolumn{2}{|l|}{ F/Name } \\
\hline \multicolumn{2}{|l|}{ Age } \\
\hline Sex & Male \\
\hline Address in Pakistan & Sadda, NWFP. Pakistan \\
\hline \multicolumn{2}{|c|}{ Address in Afghanistan } \\
\hline Accusation/Crime & He was arrested under section 40 FCR. \\
\hline \multicolumn{2}{|l|}{ Date arrested } \\
\hline Prison & Kohat \\
\hline Type of Prison & District Prison" \\
\hline Present condition & Not released. \\
\hline Remarks & \\
\hline
\end{tabular}

\begin{tabular}{|c|c|}
\hline S. No. & $09-085-189$ \\
\hline Name & Gul Bashar \\
\hline \multicolumn{2}{|l|}{ F/Name } \\
\hline \multicolumn{2}{|l|}{ Age } \\
\hline Sex & Male \\
\hline Address in Pakistan & Sadda, NWFP, Pakistan \\
\hline \multicolumn{2}{|c|}{ Address in Afghanistan } \\
\hline Accusation/Crime & He was arrested under section $40 \mathrm{FCR}$. \\
\hline \multicolumn{2}{|l|}{ Date arrested } \\
\hline Prison & Kohat \\
\hline Type of Prison & District Prison \\
\hline Present condition & Not released. \\
\hline Remarks & \\
\hline
\end{tabular}




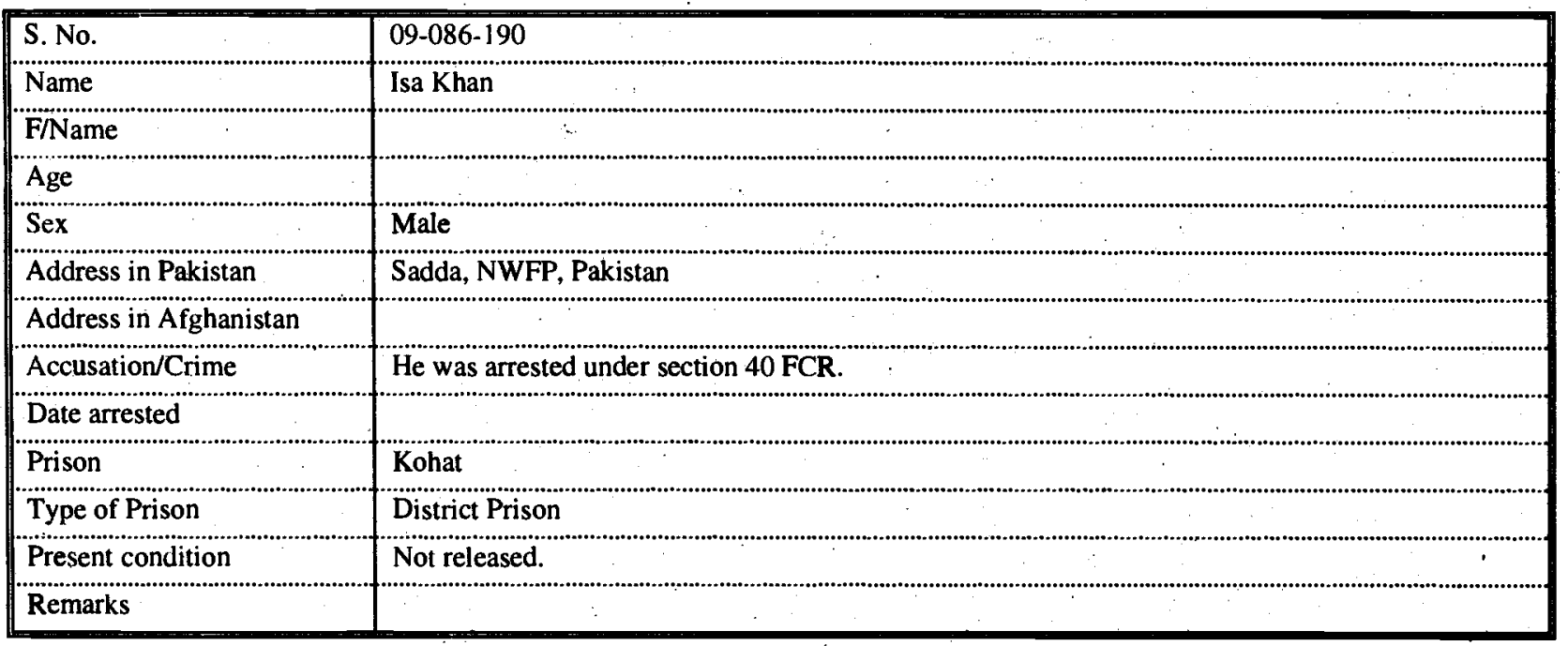

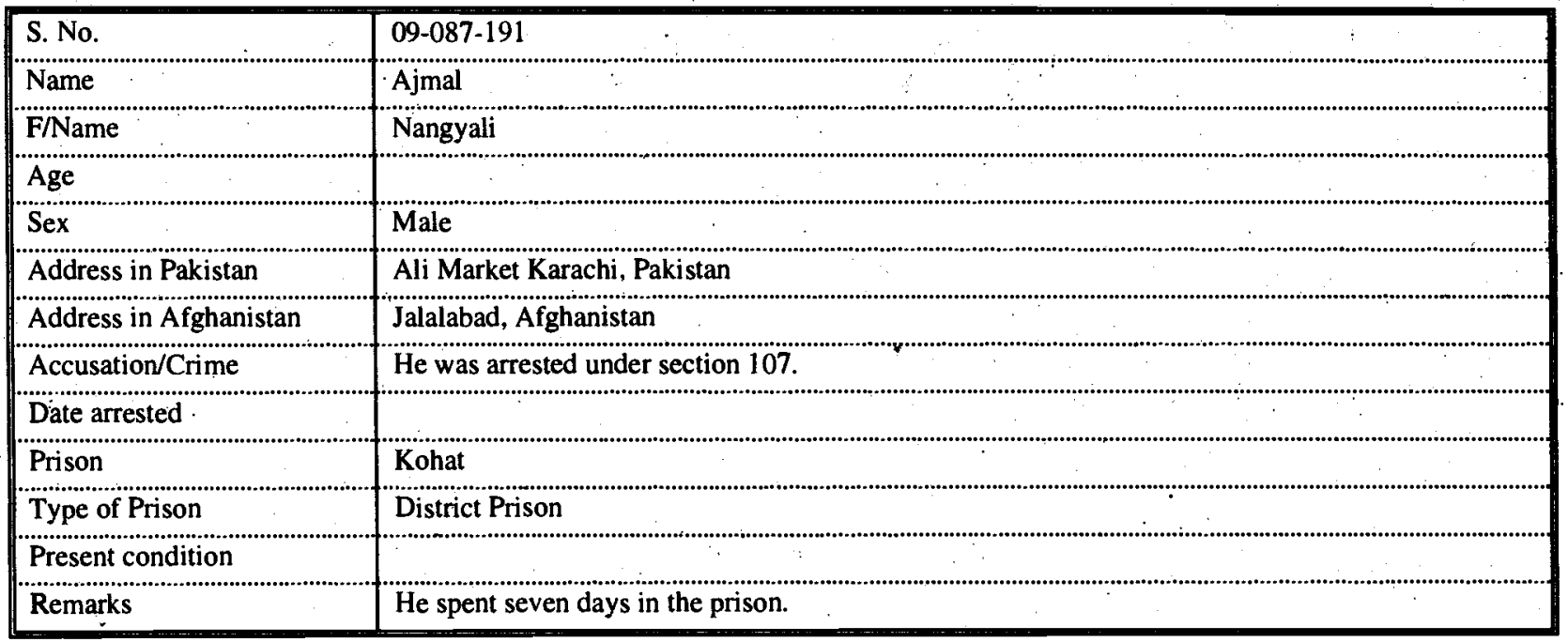

\begin{tabular}{|c|c|}
\hline S. No. & $09-088-192$ \\
\hline Name & Sayyed Marjan \\
\hline F/Name & Sarwar Jan \\
\hline \multicolumn{2}{|l|}{ Age } \\
\hline Sex & Male \\
\hline Address in Pakistan & Tora Wala, NWFP, Pakistan \\
\hline Accusation/Crime & He was arrested under section 428/424 PPC. \\
\hline Date arrested & \\
\hline Prison & Kohat \\
\hline Type of Prison & Districe Prison \\
\hline
\end{tabular}




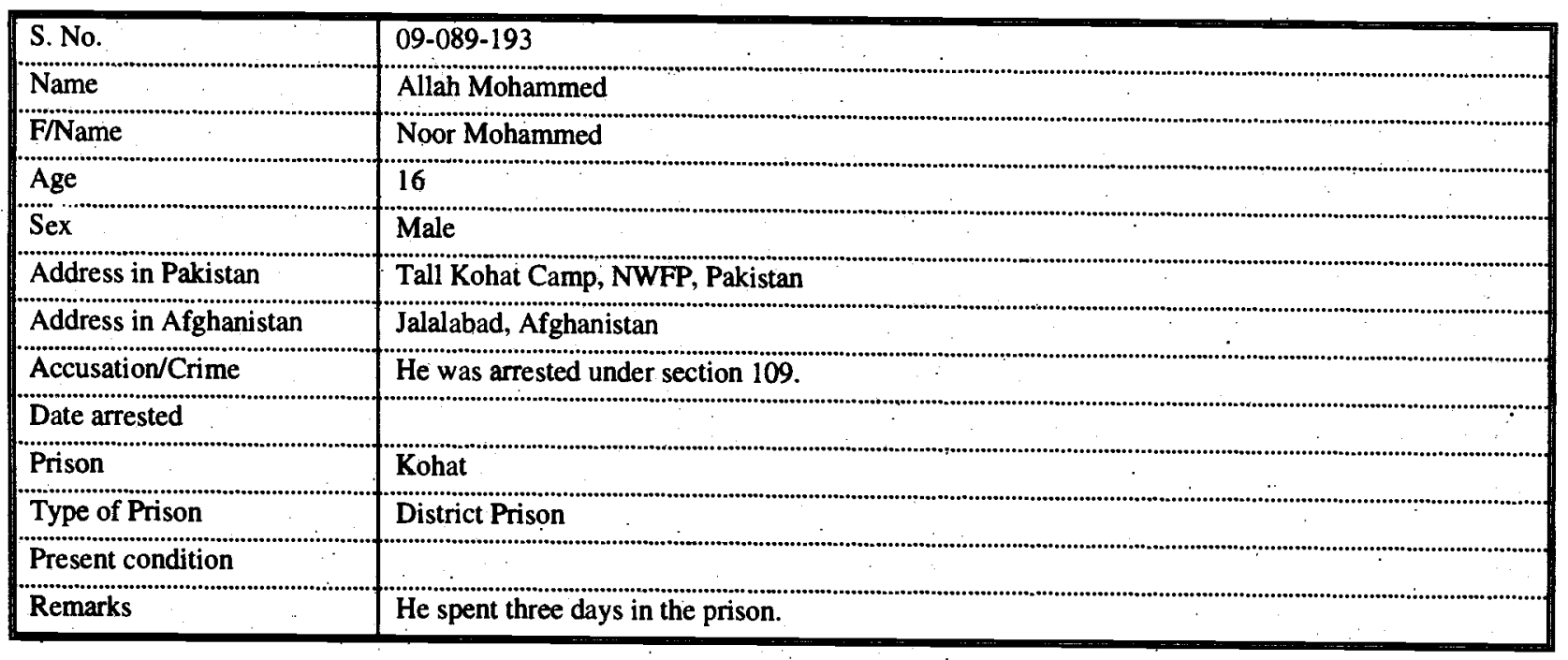

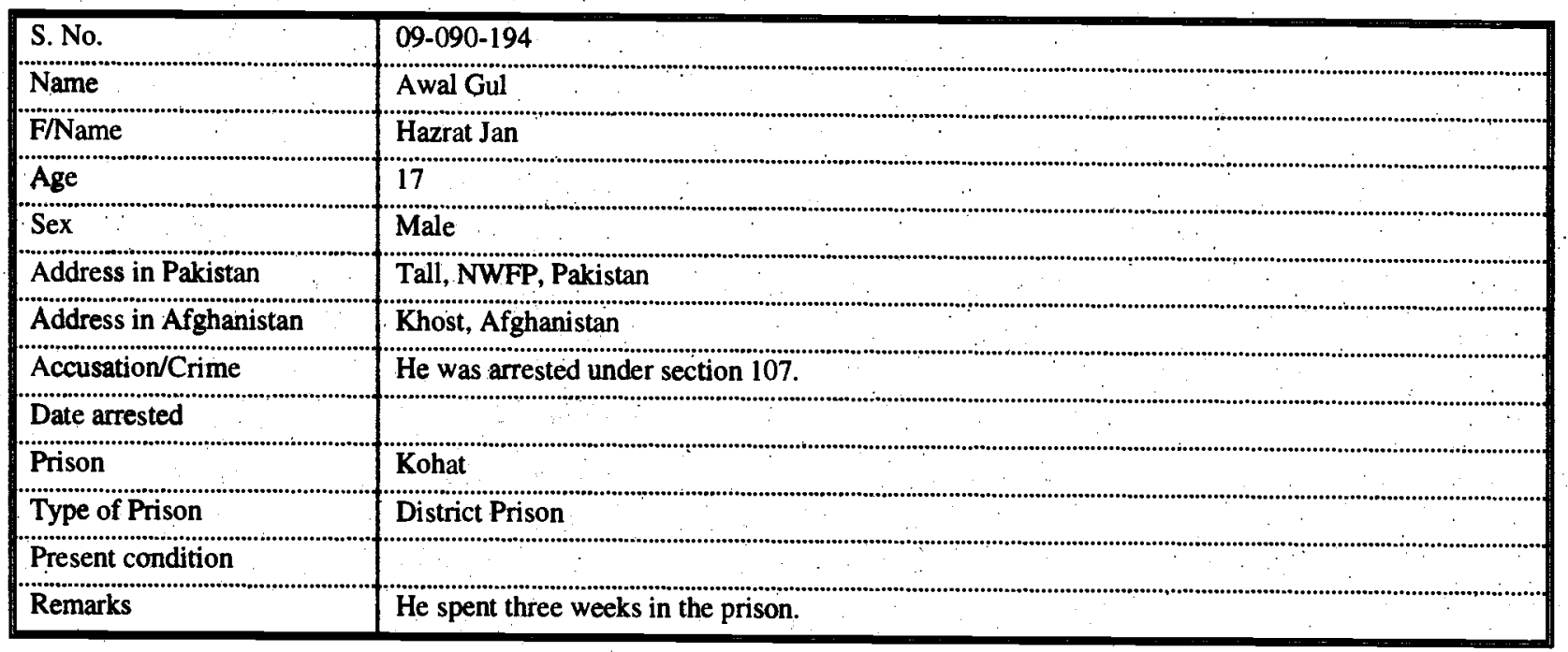

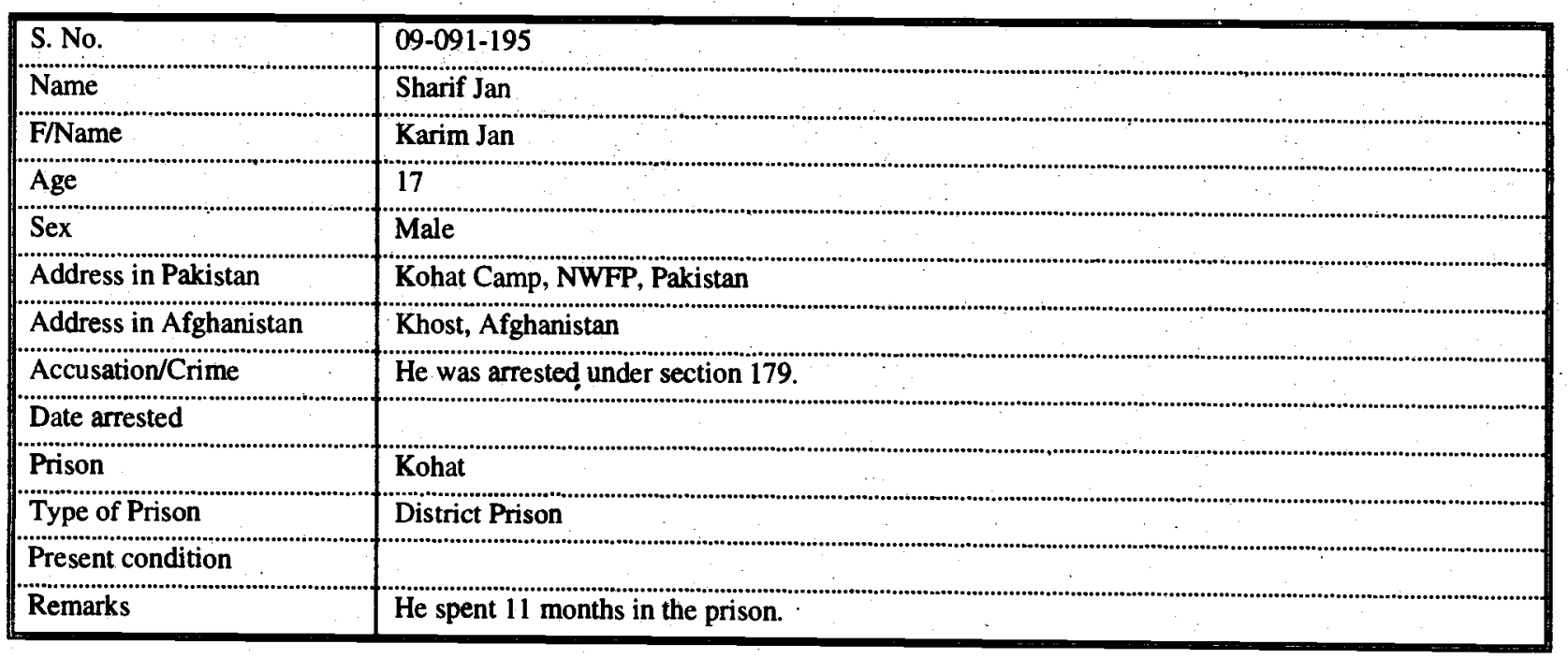




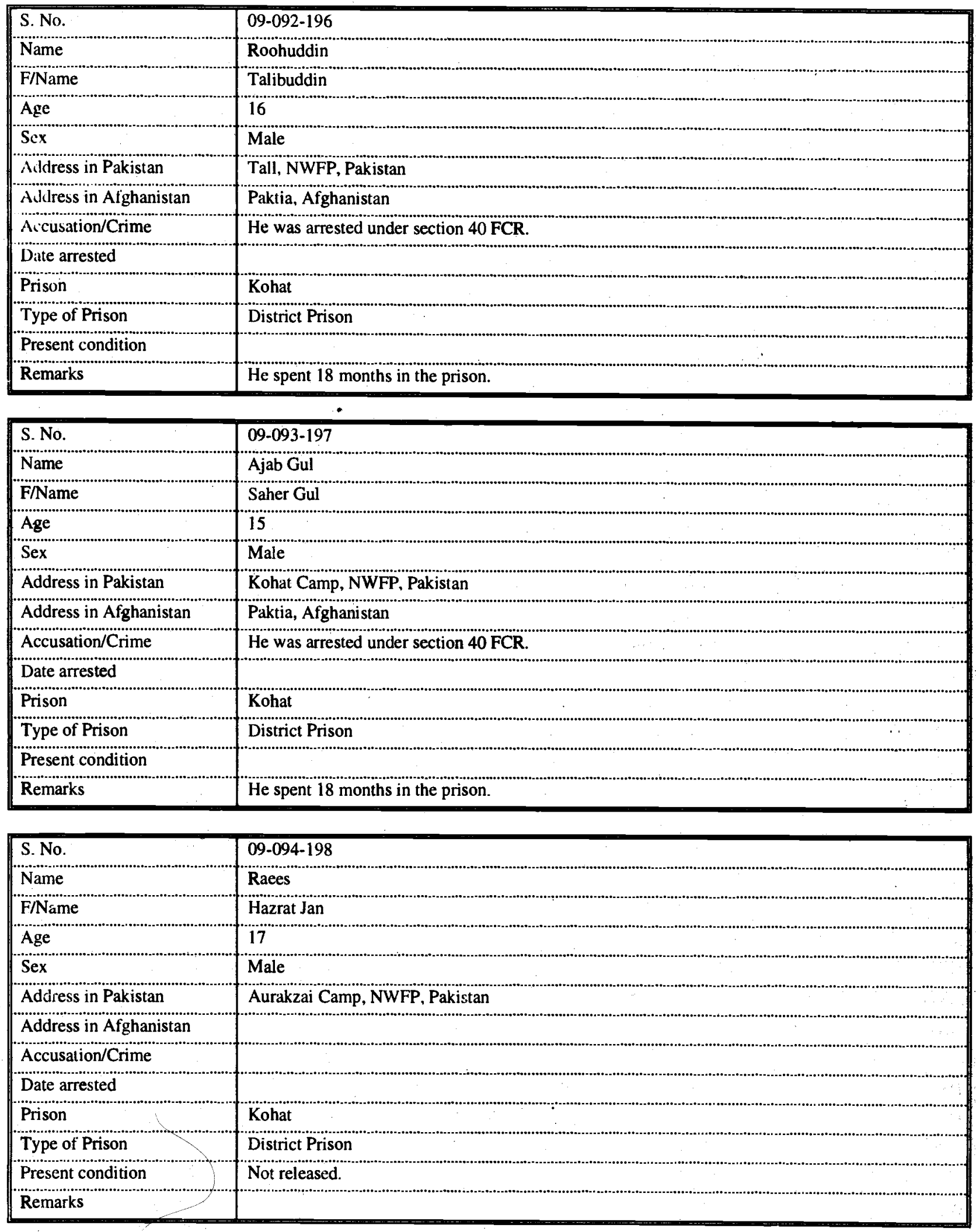




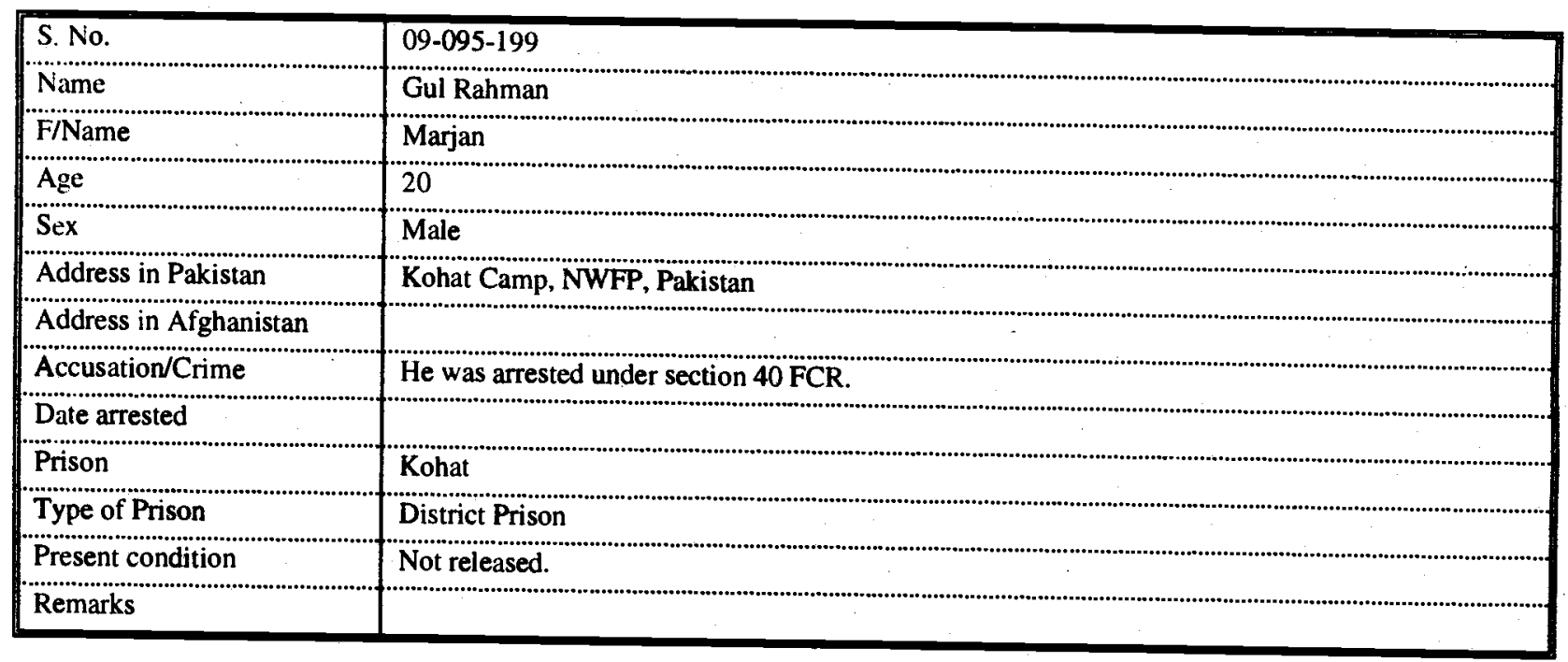

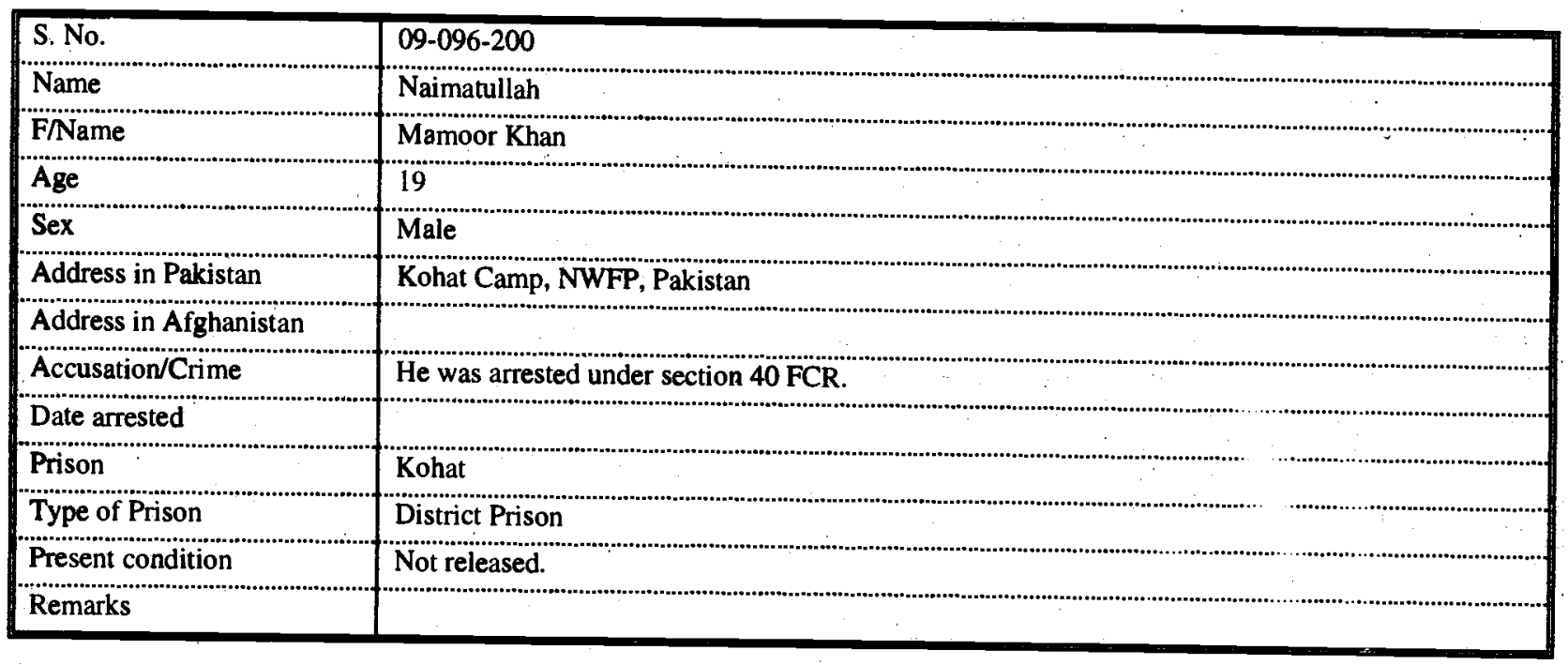

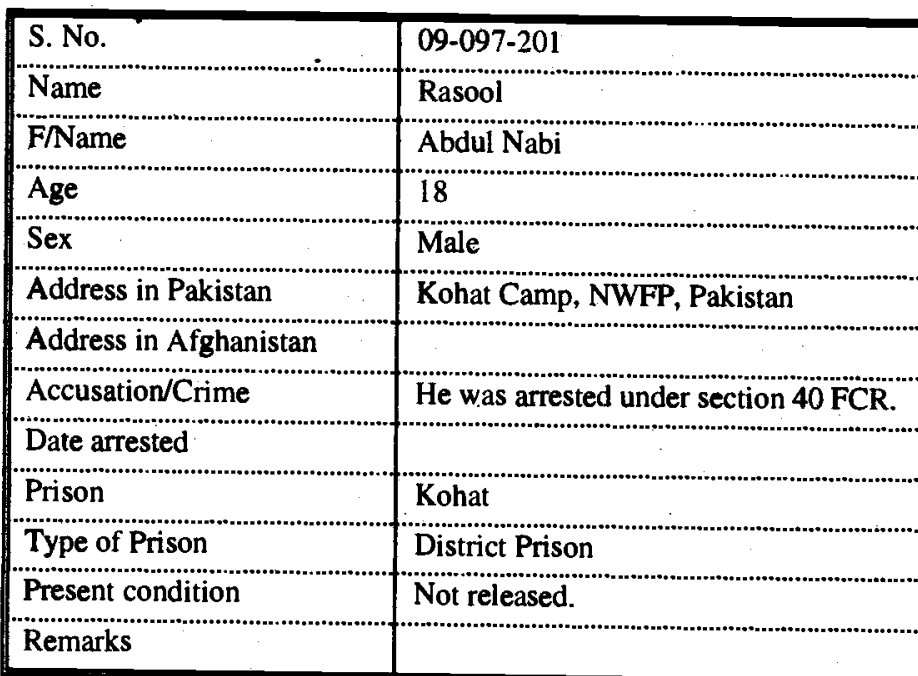




\begin{tabular}{|c|c|}
\hline S. No. & 09-098-202 \\
\hline Name & Bismellah \\
\hline F/Name & Noor Khan \\
\hline Age & 19 \\
\hline Sex & Male \\
\hline Address in Pakistan & Lachi, NWFP, Pakistan \\
\hline Address in Afghanistan & \\
\hline Accusation/Crime & He was arrested under section 40 FCR. \\
\hline Date arrested & \\
\hline Prison & Kohat \\
\hline Type of Prison & District Prison \\
\hline Present condition & Not released. \\
\hline Remarks & \\
\hline
\end{tabular}

\begin{tabular}{|c|c|}
\hline S. No. & 09-099-203 \\
\hline Name & Suleman \\
\hline F/Name & Nazir. \\
\hline Age & 22 \\
\hline Sex & Male \\
\hline Address in Pakistan & Kohat, NWFP, Pakistan \\
\hline \multicolumn{2}{|l|}{ Address in Afghanistan } \\
\hline Accusation/Crime & He was arrested under section different cases. \\
\hline \multicolumn{2}{|l|}{ Date arrested } \\
\hline Prison & Kohat \\
\hline Type of Prison & District Prison \\
\hline Present condition & Not released. \\
\hline Remarks & \\
\hline
\end{tabular}

\begin{tabular}{|l|l|}
\hline S. No. & $09-100-204$ \\
\hline Name & Dowlat Khan \\
\hline F/Name & Ghulam Dastagir \\
\hline Age & 21 \\
\hline Sex & Male \\
\hline Address in Pakistan & Hango, NWFP, Pakistan \\
\hline Address in Afghanistan & Laghaman, Afghanistan \\
\hline Accusation/Crime & He was arrested under section 107. \\
\hline Date arrested & Kohat \\
\hline Prison & District Prison \\
\hline Type of Prison & He spent seven days in the prison. \\
\hline Present condition & \\
\hline Remarks &
\end{tabular}




\begin{tabular}{|c|c|}
\hline S. No. & 09-101-205 \\
\hline Name & Faiz Mohammed. \\
\hline F/Name & Noor Mohammed \\
\hline Age & 21 \\
\hline Sex & Male \\
\hline Address in Pakistan & Kohat, NWFP, Pakistan \\
\hline Address in Afghanistan & Logar, Afghanistan \\
\hline Accusation/Crime & He was arrested under section 337 . \\
\hline Date arrested & 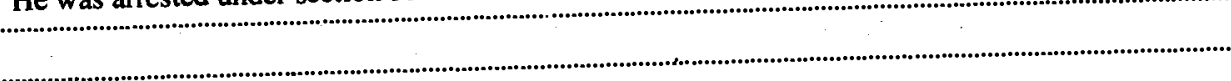 \\
\hline Prison & Kohat \\
\hline Type of Prison & District Prison \\
\hline Present condition & \\
\hline Remarks & He spent 20 days in the prison. \\
\hline
\end{tabular}

\begin{tabular}{|c|c|}
\hline S. No. & 09-102-206 \\
\hline Name & Miral Khel \\
\hline F/Name & Sayyed Hassan \\
\hline Age & Sayуё nassant \\
\hline Sex & Male \\
\hline Address in Pakistan & Kohat, NWFP, Pakistan \\
\hline Address in Afghanistan & Paktia, Afghanistan \\
\hline Accusation/Crime & He was arrested under section 107 . \\
\hline Date arrested & \\
\hline Prison & Kohat \\
\hline Type of Prison & District Prison \\
\hline Present condition & \\
\hline Remarks & He spent 9 days in the prison. \\
\hline
\end{tabular}

\begin{tabular}{|c|c|}
\hline S. No. & $09-103-207$ \\
\hline Name' & Bismallah \\
\hline FiName & Ramit Mir \\
\hline Age & 35 \\
\hline Sex & Male \\
\hline Address in Pakistan & Kohat, NWFP, Pakistan \\
\hline Address in Afghanistan & Kundoz City, Afghanistan \\
\hline Accusation/Crime & He was arrested under section 4 PO. \\
\hline \multicolumn{2}{|l|}{ Date arrested } \\
\hline Prisơr & Kohat \\
\hline Type of Prison & District Prison \\
\hline \multicolumn{2}{|l|}{ Present condition } \\
\hline Remarks & He speni 9 months in the prison. \\
\hline
\end{tabular}




\begin{tabular}{|c|c|}
\hline S. No. & $09-104-208$ \\
\hline Name & Naeem \\
\hline F/Name & Mahmood \\
\hline Age & 17 \\
\hline Sex & Male \\
\hline Address in Pakistan & Kohat, NWFP, Pakistan \\
\hline Address in Afghanistan & Paktia, Afghanistan \\
\hline Accusation/Crime & He was arrested under section 40 FCR. \\
\hline \multicolumn{2}{|l|}{ Date arrested } \\
\hline Prison & Kohat \\
\hline Type of Prison & District Prison \\
\hline Present condition & Not released. \\
\hline Remarks & \\
\hline
\end{tabular}

\begin{tabular}{|c|c|}
\hline S. No. & $09-105-209$ \\
\hline Name & Sur Gul \\
\hline F/Name & Wazir Gul \\
\hline Age & 70 \\
\hline Sex & Male \\
\hline Address in Pakistan & Duaba, NWFP, Pakistan \\
\hline Address in Afghanistan & Paktia, Afghanistan \\
\hline Accusation/Crime & He was arrested under section 40 FCR. \\
\hline Date arrested & Q \\
\hline Prison & Kohat \\
\hline Type of Prison & District Prison \\
\hline Present condition & Not released. \\
\hline Remarks & \\
\hline
\end{tabular}

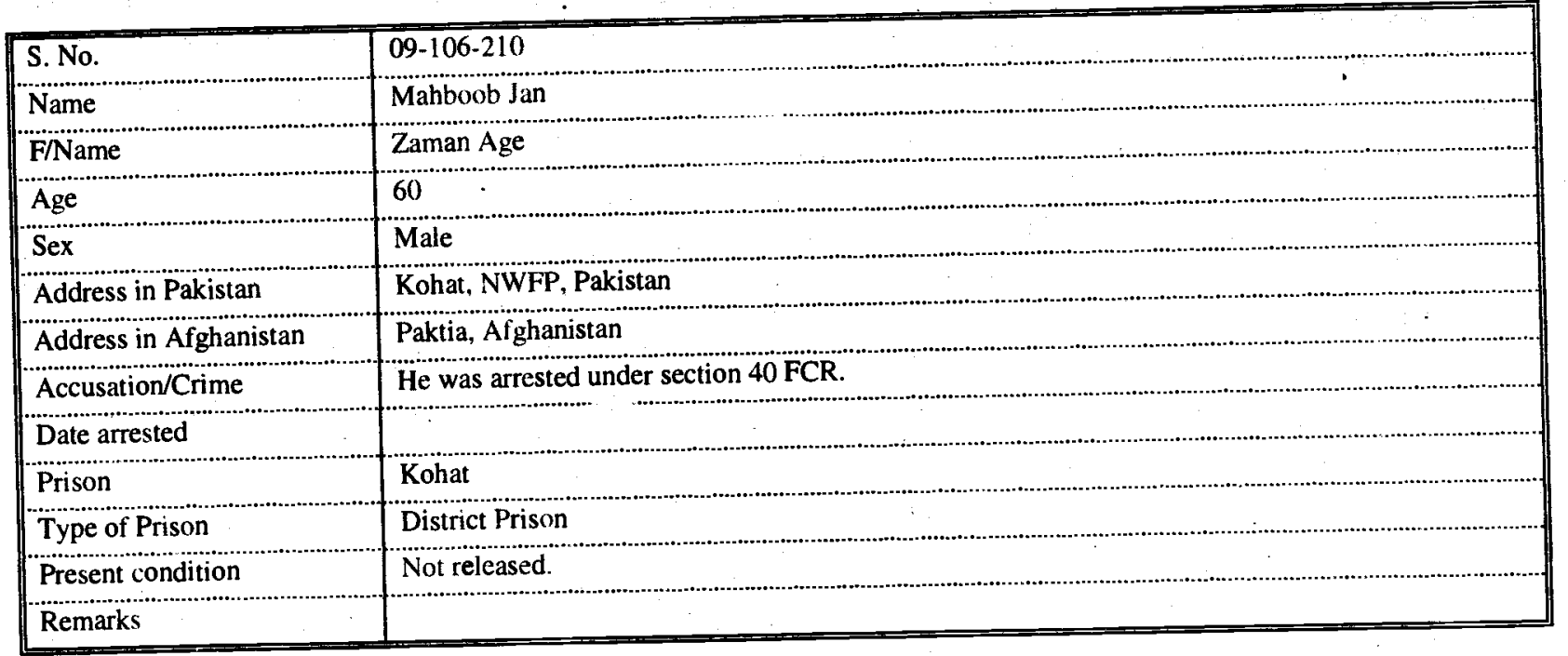




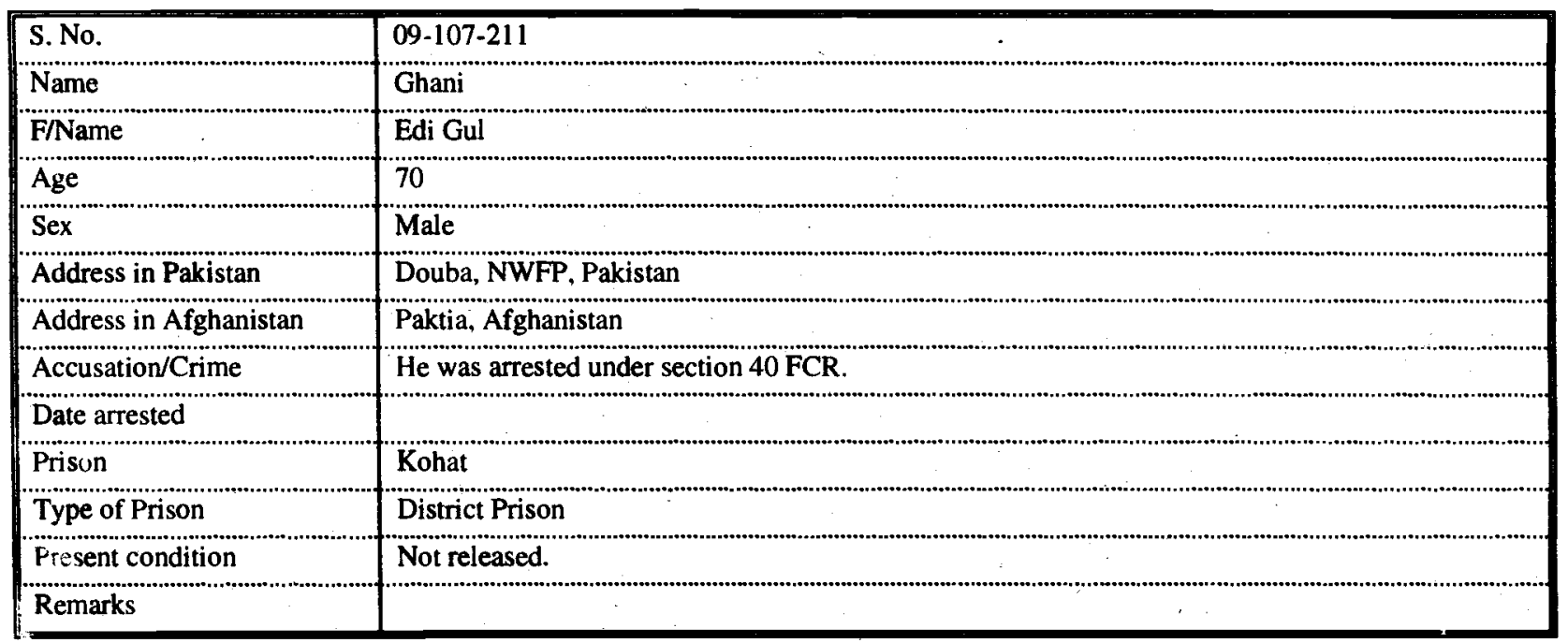

\begin{tabular}{|c|c|}
\hline S. No. & 09-108-212 \\
\hline Name & Tahir \\
\hline F/Name & Sardar Shah \\
\hline Age & 28 \\
\hline Sex & Male \\
\hline Address in Pakistan & Kohat, NWFP, Pakistan \\
\hline Address in Afghanistan & Paktia, Afghanistan \\
\hline Accusation/Crime & He was arrested under section 40 FCR. \\
\hline \multicolumn{2}{|l|}{ Date arrested } \\
\hline Prison & Kohat \\
\hline Type of Prison & District Prison \\
\hline Present condition & Not released. \\
\hline Remarks & \\
\hline
\end{tabular}

\begin{tabular}{|l|l|l|}
\hline S. No. & $09-109-213$ \\
\hline Name & Karim & Zar Khan \\
\hline F/Name & 26 & Male \\
\hline Age & Kohat, NWFP, Pakistan \\
\hline Sex & Paktia, Afghanistan \\
\hline Address in Pakistan & He was arrested under section 40 FCR \\
\hline Address in Afghanistan & Kohat \\
\hline Accusation/Crime & Disirict Prison \\
\hline Date arrested & Not released. \\
\hline Prison & \\
\hline Type of Prison & \\
\hline Present condition & \\
\hline Remarks & & \\
\hline
\end{tabular}




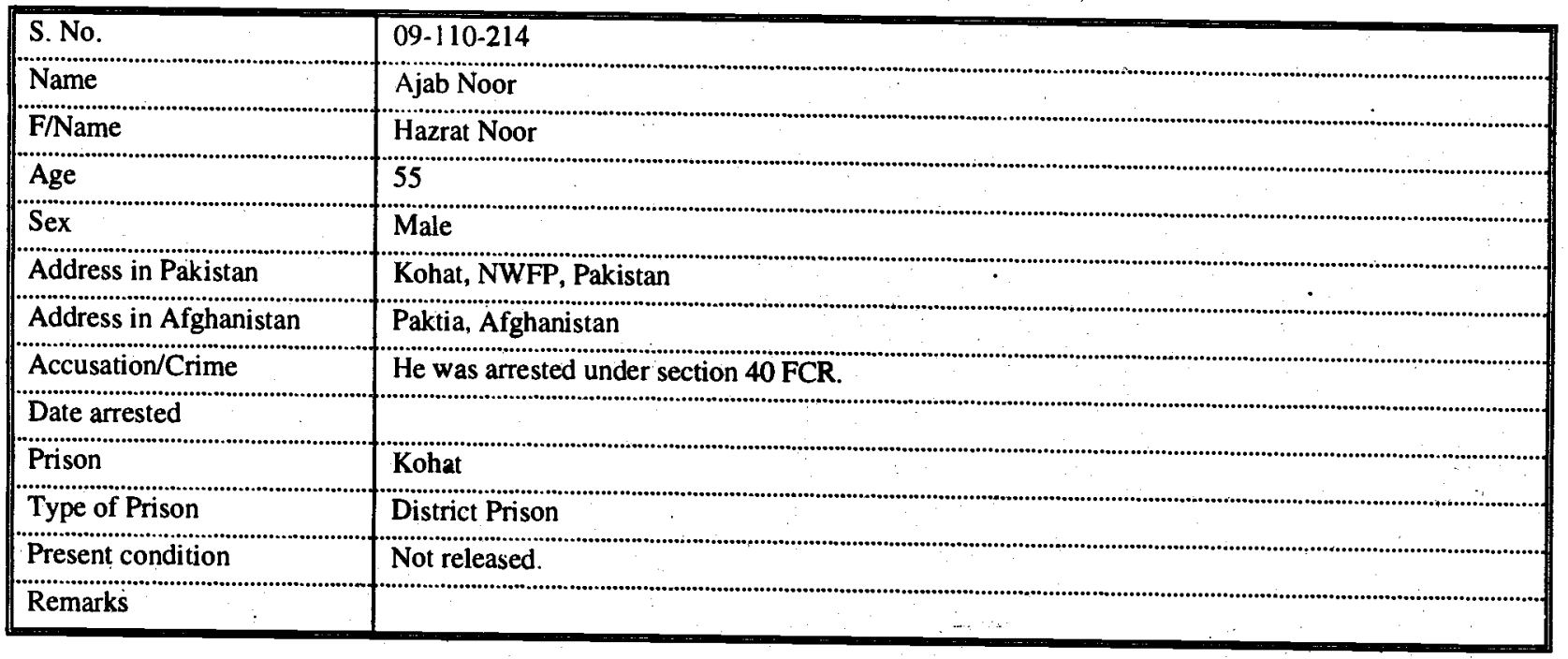

\begin{tabular}{|c|c|}
\hline S. No. & $09-111-215$ \\
\hline Name & Allozia \\
\hline F/Name & Faiz Mohammed \\
\hline Age & 35 \\
\hline Sex & Male \\
\hline Address in Pakistan & Duaba, NWFP, Pakistan \\
\hline Address in Afghanistan & Paktia, Afghanistan \\
\hline Accusation/Crime & He was arrested under section $40 \mathrm{FCR}$. \\
\hline \multicolumn{2}{|l|}{ Date arrested } \\
\hline Prison & Kohat \\
\hline Type of Prison & District Prison \\
\hline Present condition & Not released. \\
\hline Remarks & \\
\hline
\end{tabular}

\begin{tabular}{|c|c|}
\hline S. No. & $09-112-216$ \\
\hline Name & Sayyed Mohammed \\
\hline F/Name & Taza Gul \\
\hline Age & 35 \\
\hline Sex & Male \\
\hline Address in Pakistan & Lachi, NWFP, Pakistan \\
\hline Address in Afghanistan & Paktia, Afghanistan \\
\hline Accusation/Crime & He was arrested under section $40 \mathrm{FCR}$. \\
\hline Date arrested & \\
\hline Prison & Kohat \\
\hline Type of Prison & District Prison \\
\hline Present condition & Not released. \\
\hline Remarks & \\
\hline
\end{tabular}




\begin{tabular}{|c|c|}
\hline S. No. & 09-113-217 \\
\hline Name & Yunus \\
\hline F/Name & Amir Mohammed \\
\hline Age & 30 \\
\hline Sex & Male \\
\hline Address in Pakistan & Kohat, NWFP, Pakistan \\
\hline Address in Afghanistan & Nangarhar, Afghanistan \\
\hline Accusation/Crime & He was arrested under section $107 / 360$. \\
\hline \multicolumn{2}{|l|}{ Date arrested } \\
\hline Prison & Kohat \\
\hline Type of Prison & District Prison \\
\hline \multicolumn{2}{|l|}{ Present condition } \\
\hline Remarks & He spent 4 months in the prison. \\
\hline
\end{tabular}

\begin{tabular}{|c|c|}
\hline S. No. & $09-114-218$ \\
\hline Name & Janat Mir \\
\hline F/Name & Khan Mir \\
\hline Age & 30 \\
\hline Sex & Male \\
\hline Address in Pakistan & Kohat, NWFP, Pakistan \\
\hline Address in Afghanistan & Paktia, Afghanistan \\
\hline Accusation/Crime & He was arrested under section 40 FÇR. \\
\hline \multicolumn{2}{|l|}{ Date arrested } \\
\hline Prison & Kohat \\
\hline Type of Prison & District Prison \\
\hline Present condition & Not released. \\
\hline Remarks & \\
\hline
\end{tabular}

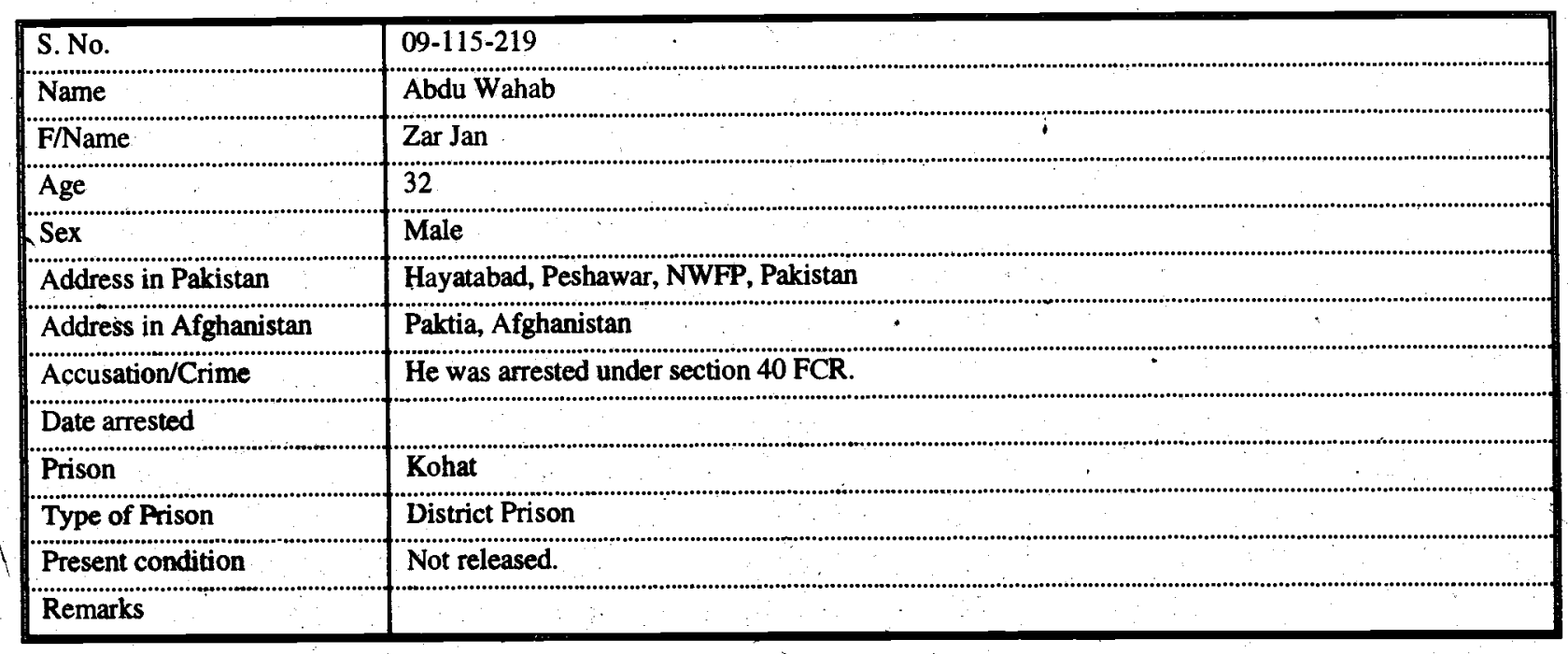




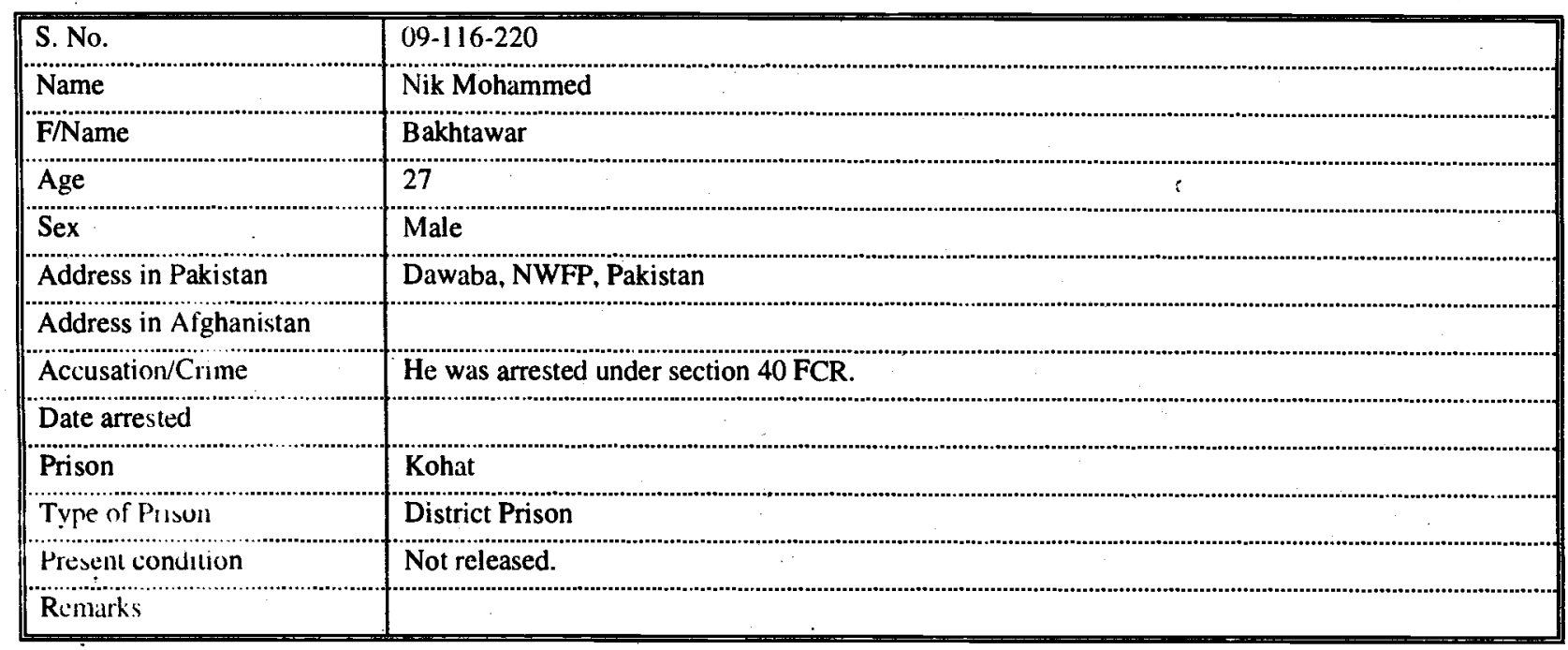

\begin{tabular}{|c|c|}
\hline S. No. & $09-117-221$ \\
\hline Name & Dawar Gul \\
\hline F/Name & Muslih \\
\hline Age & 25 \\
\hline Sex & Male \\
\hline Accusation/Crime & He was arrested under section 40 FCR. \\
\hline \multicolumn{2}{|l|}{ Date arrested } \\
\hline Prison & Kohat \\
\hline Type of Prison & District Prison \\
\hline
\end{tabular}

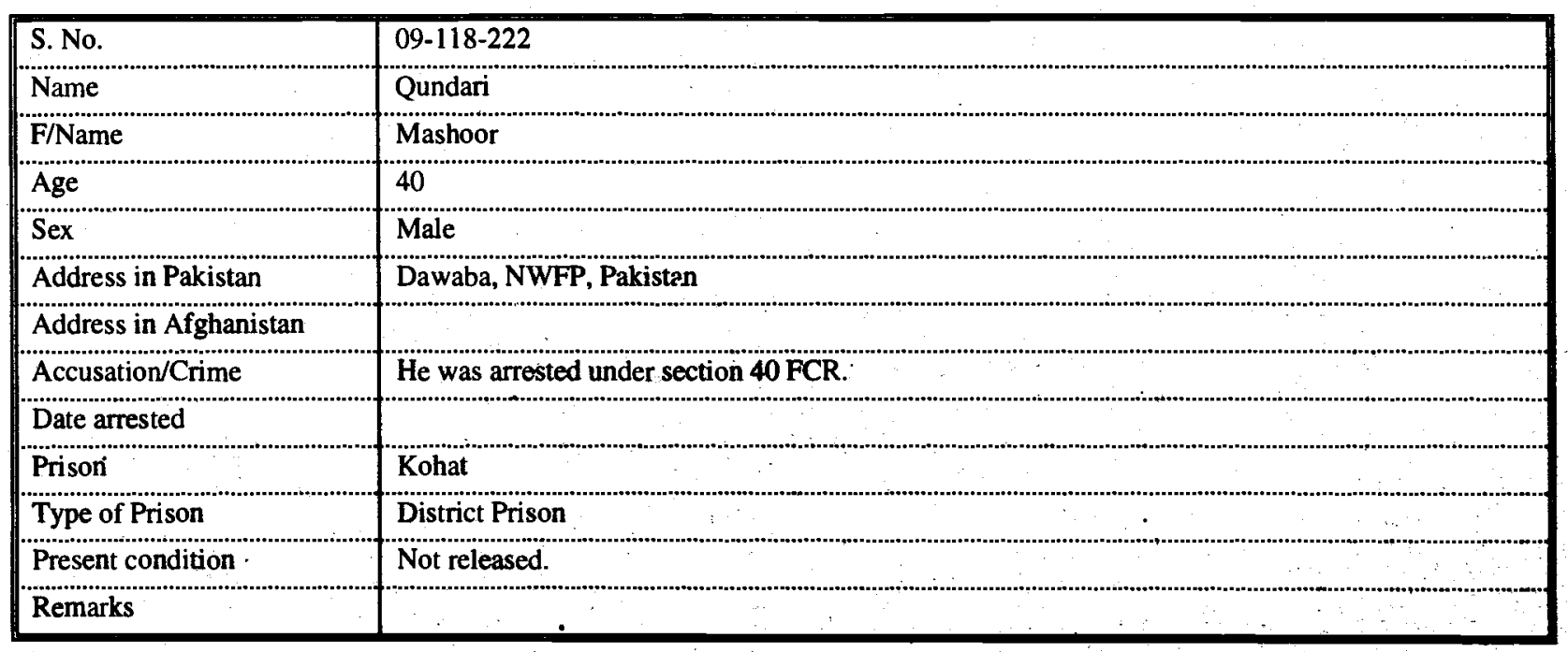




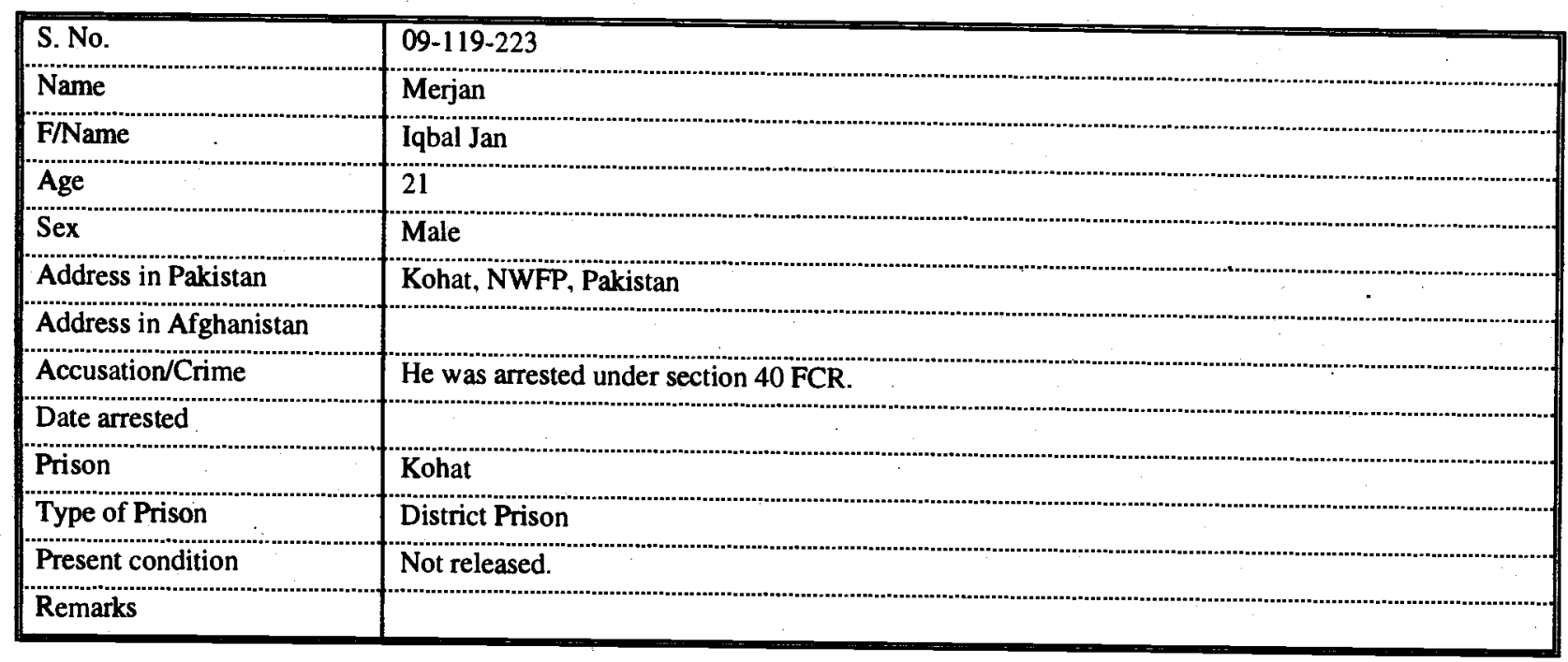

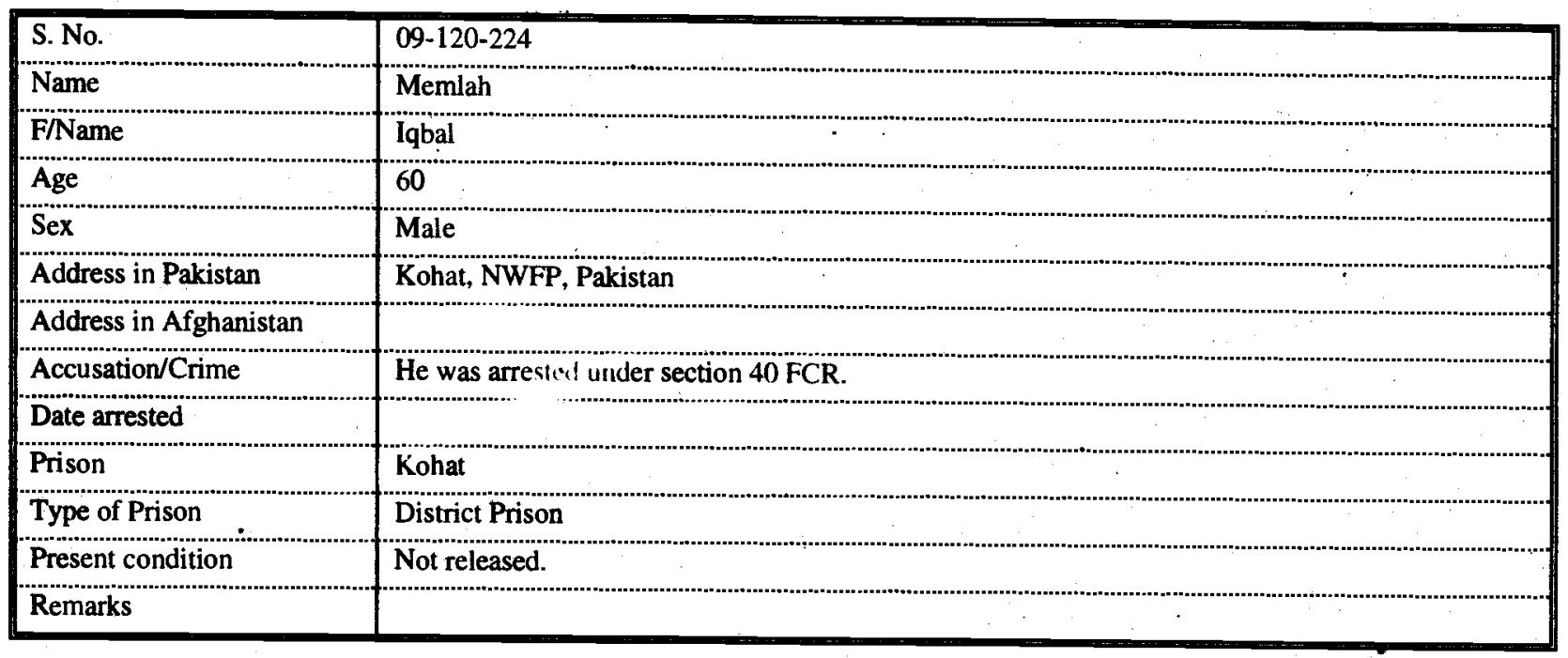

\begin{tabular}{|l|l|}
\hline S. No. & $09-121-225$ \\
\hline Name & Chinar \\
\hline F/Name & 35 \\
\hline Age & Male \\
\hline Sex & Kohat, NWFP, Pakistan \\
\hline Address in Pakistan & \\
\hline Address in Afghanistan & He was arrested under section 40 FCR. \\
\hline Accusation/Crime & Kohat \\
\hline Date arrested & District Prison \\
\hline Prison & Not released. \\
\hline Type of Prison & \\
\hline Present condition & \\
\hline Remarks & \\
\hline
\end{tabular}




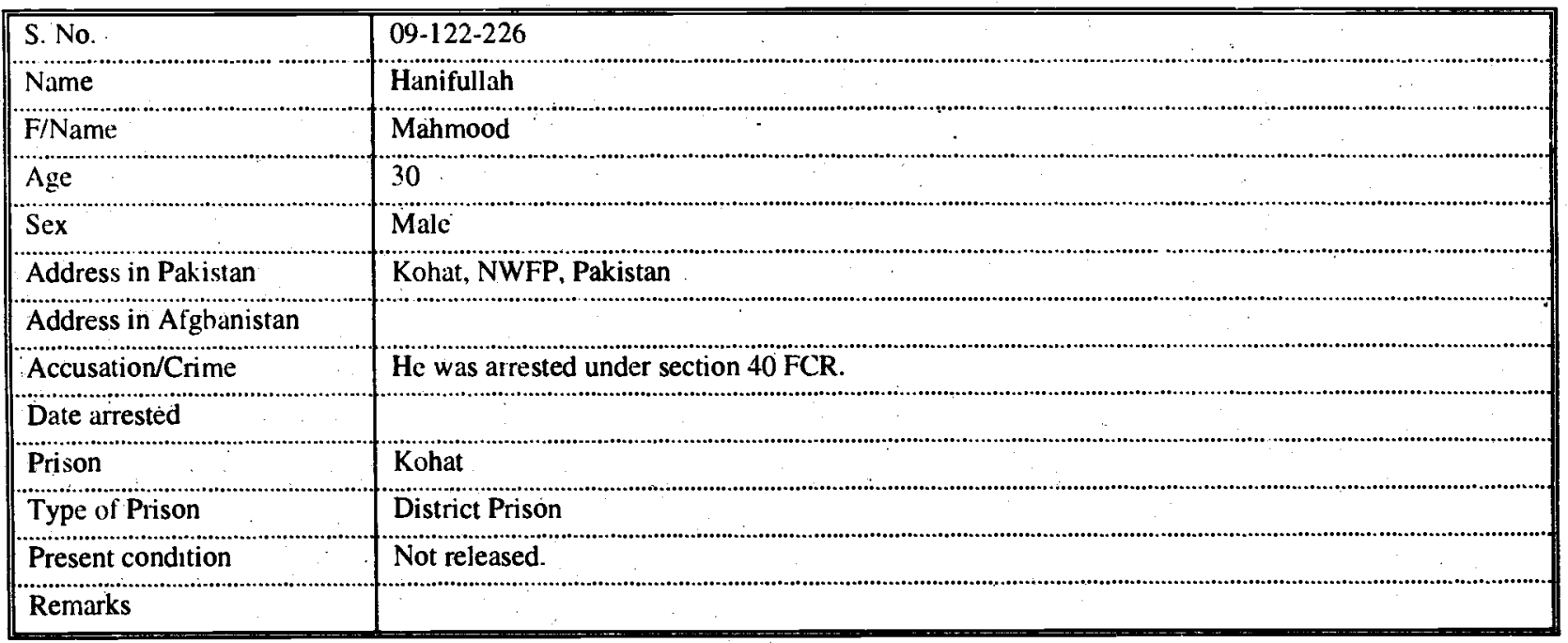

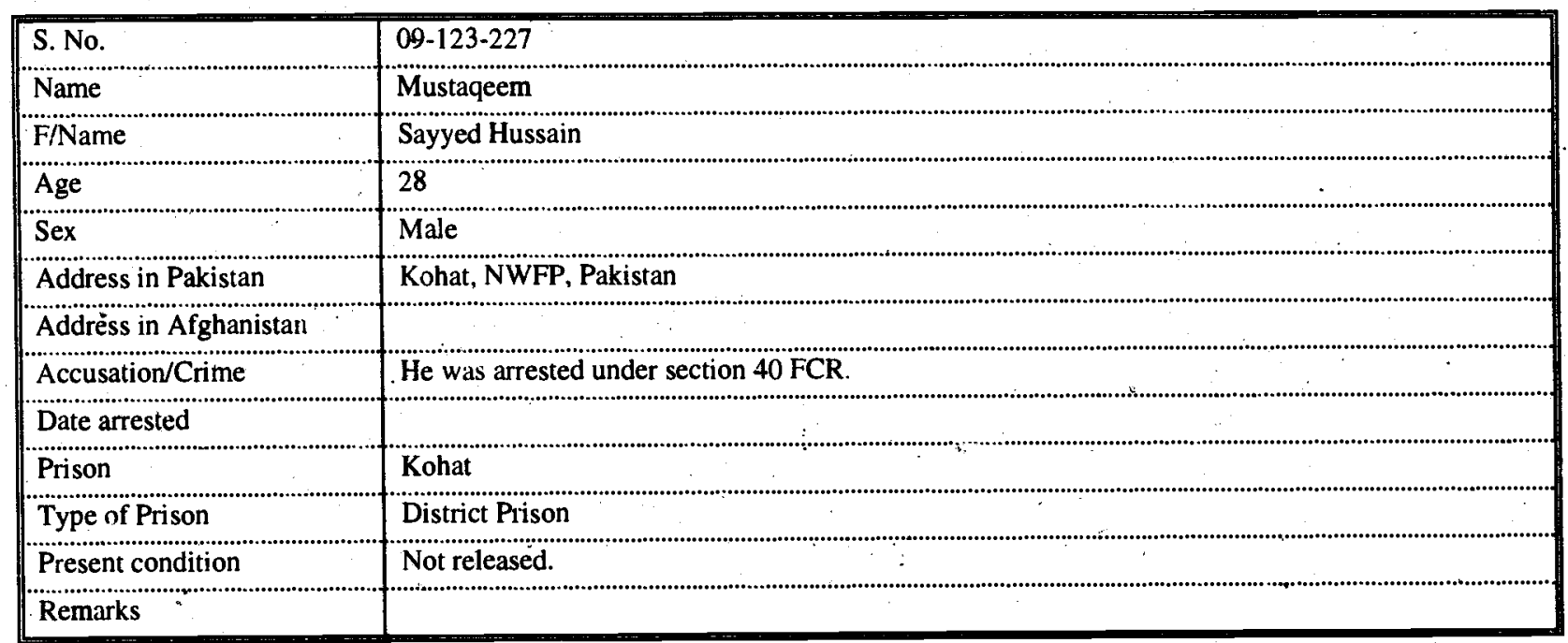

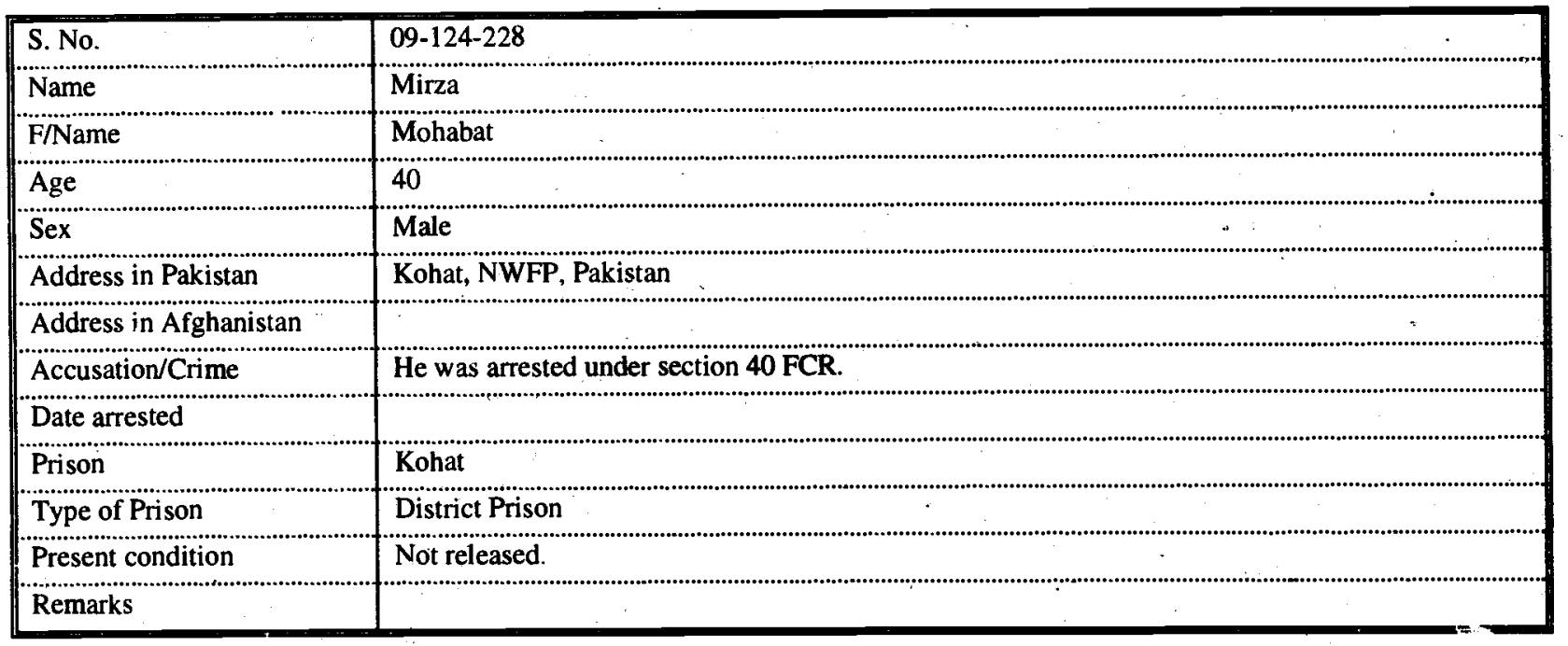




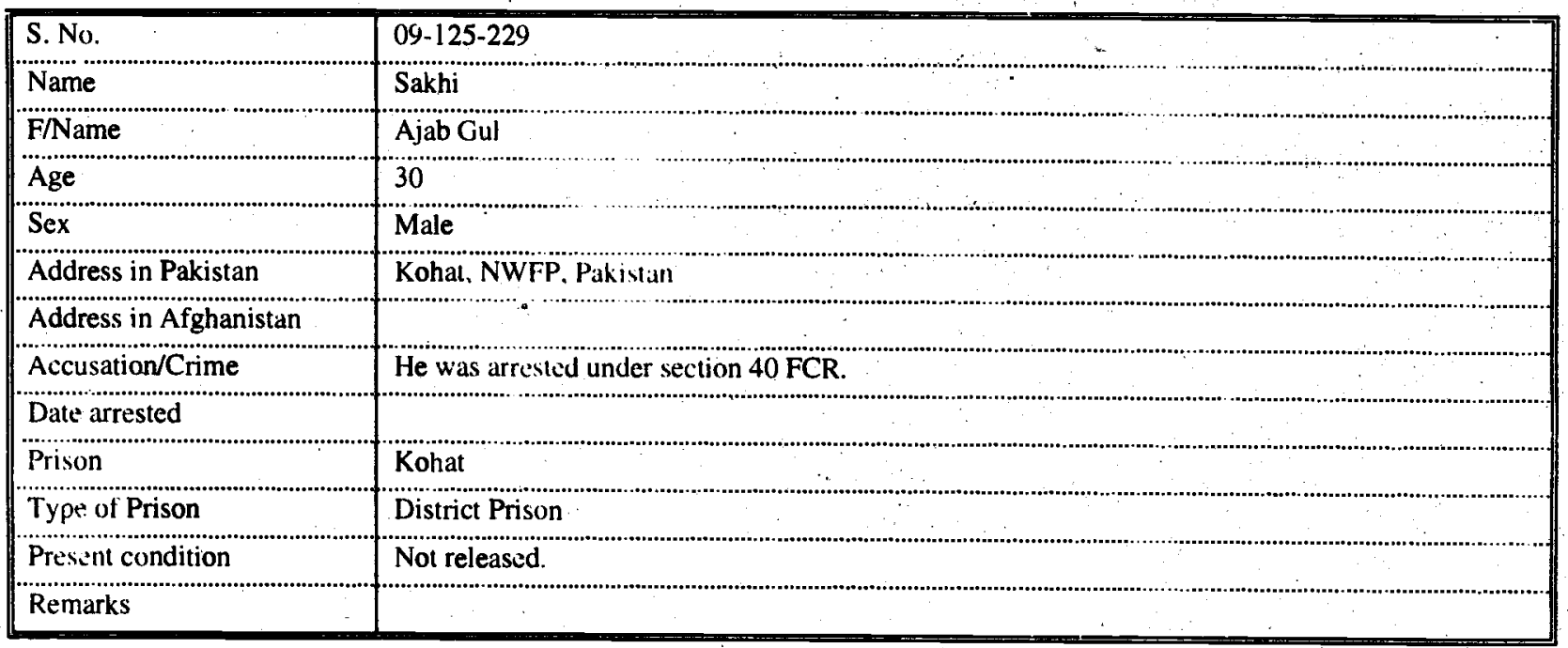

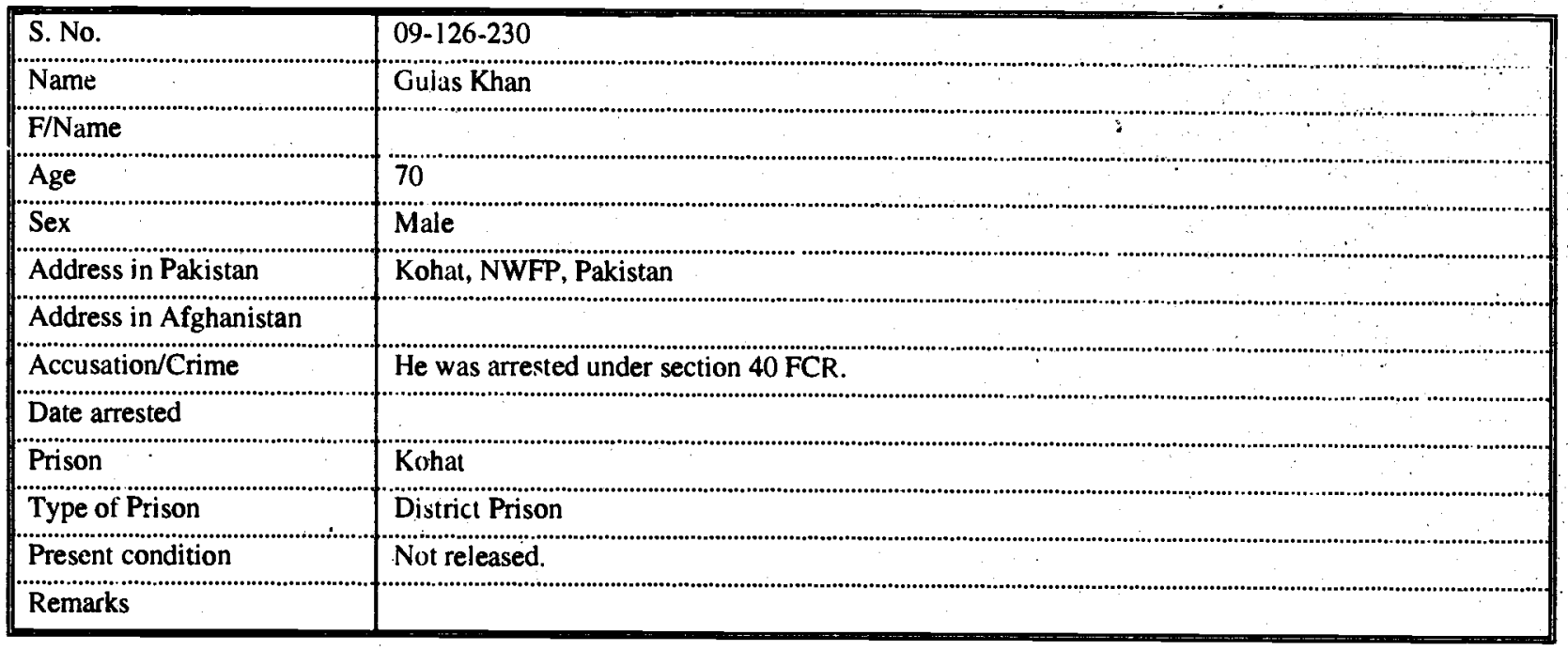

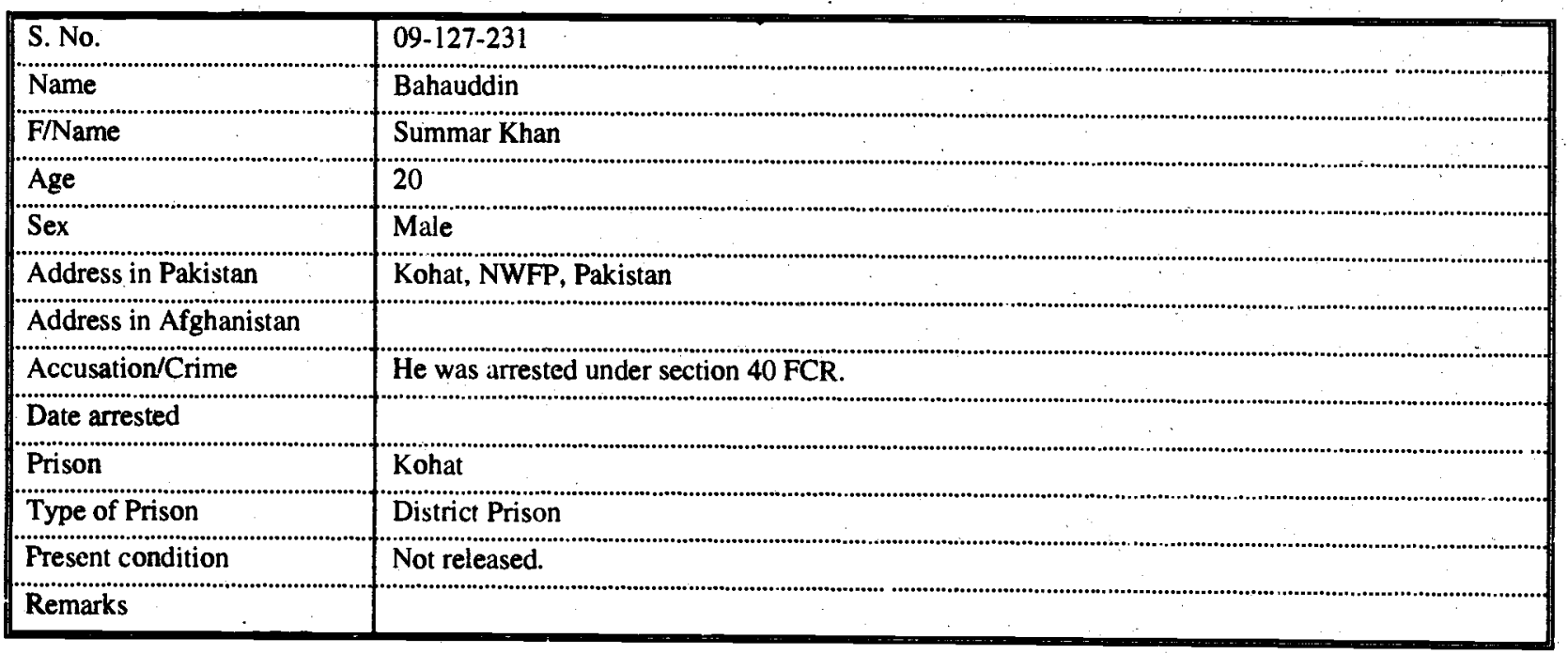




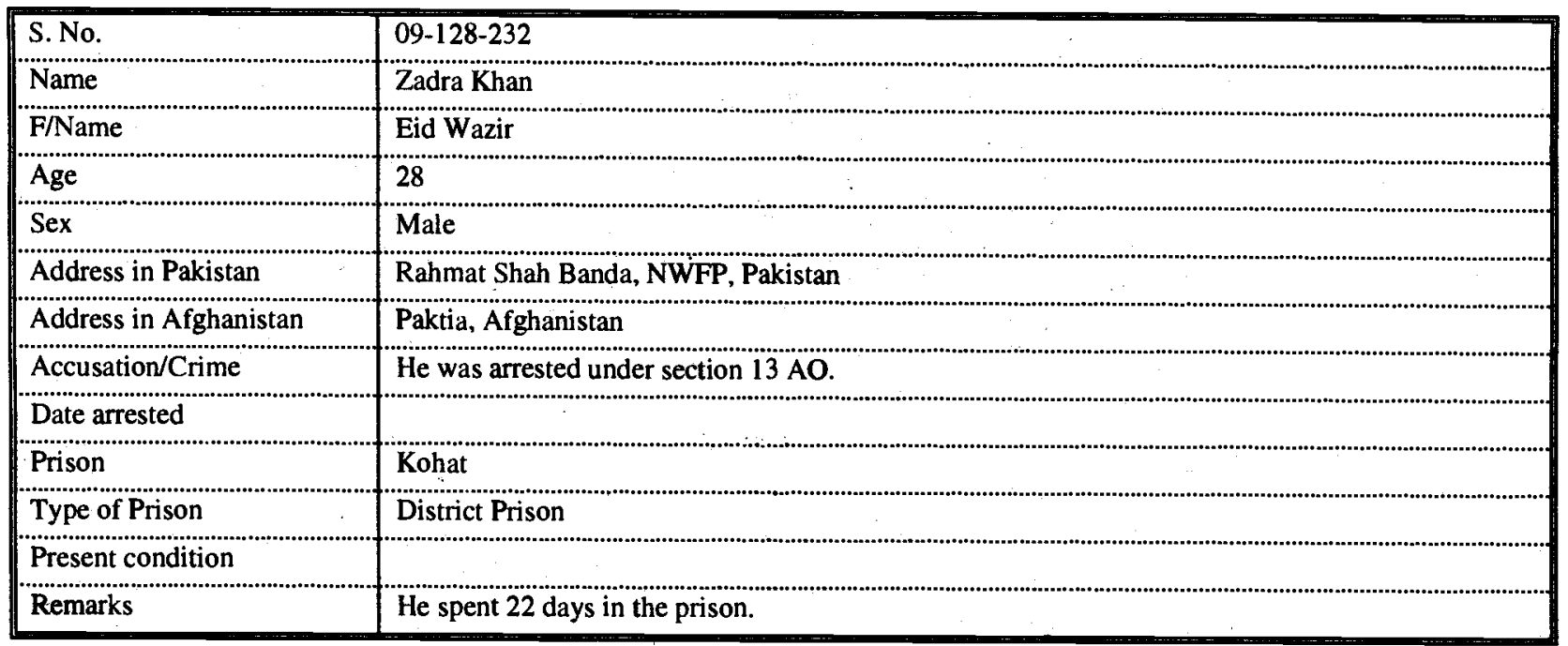




\section{District Prison Bannu}

\begin{tabular}{|c|c|}
\hline S. No. & $10-01-233$ \\
\hline Name & Kalam Din \\
\hline F/Name & Aslam Khan \\
\hline Age & 16 \\
\hline Sex & Male \\
\hline Address in Pakistan & Jhuwar Khail Pezo, NWFP, Pakistan \\
\hline Address in Afghanistan & Khost, Afghanistan \\
\hline Accusation/Crime & He was arrested under section $302 / 324$. \\
\hline \multicolumn{2}{|l|}{ Date arrested } \\
\hline Prison & Bannu \\
\hline Type of Prison & District Prison \\
\hline \multicolumn{2}{|l|}{ Present condition } \\
\hline Remarks & He spent 4 months in the prison. \\
\hline
\end{tabular}




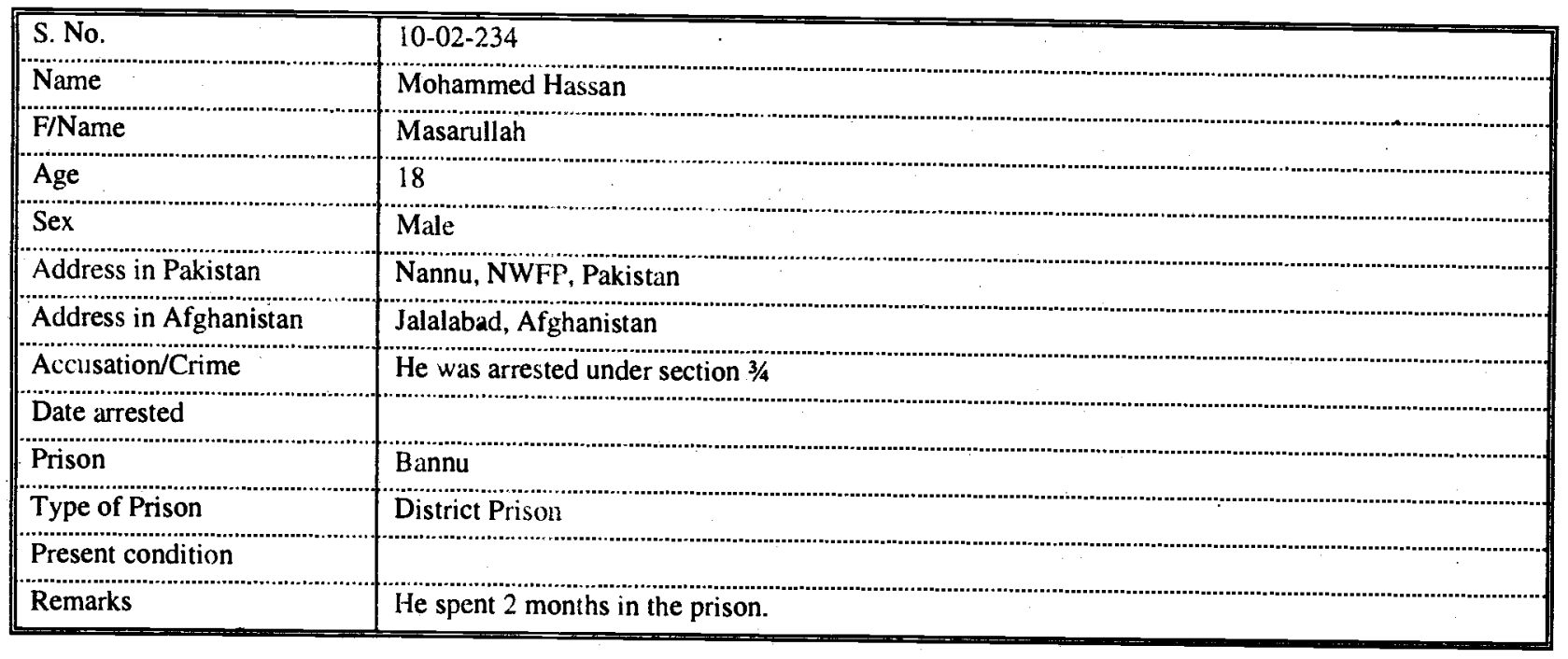

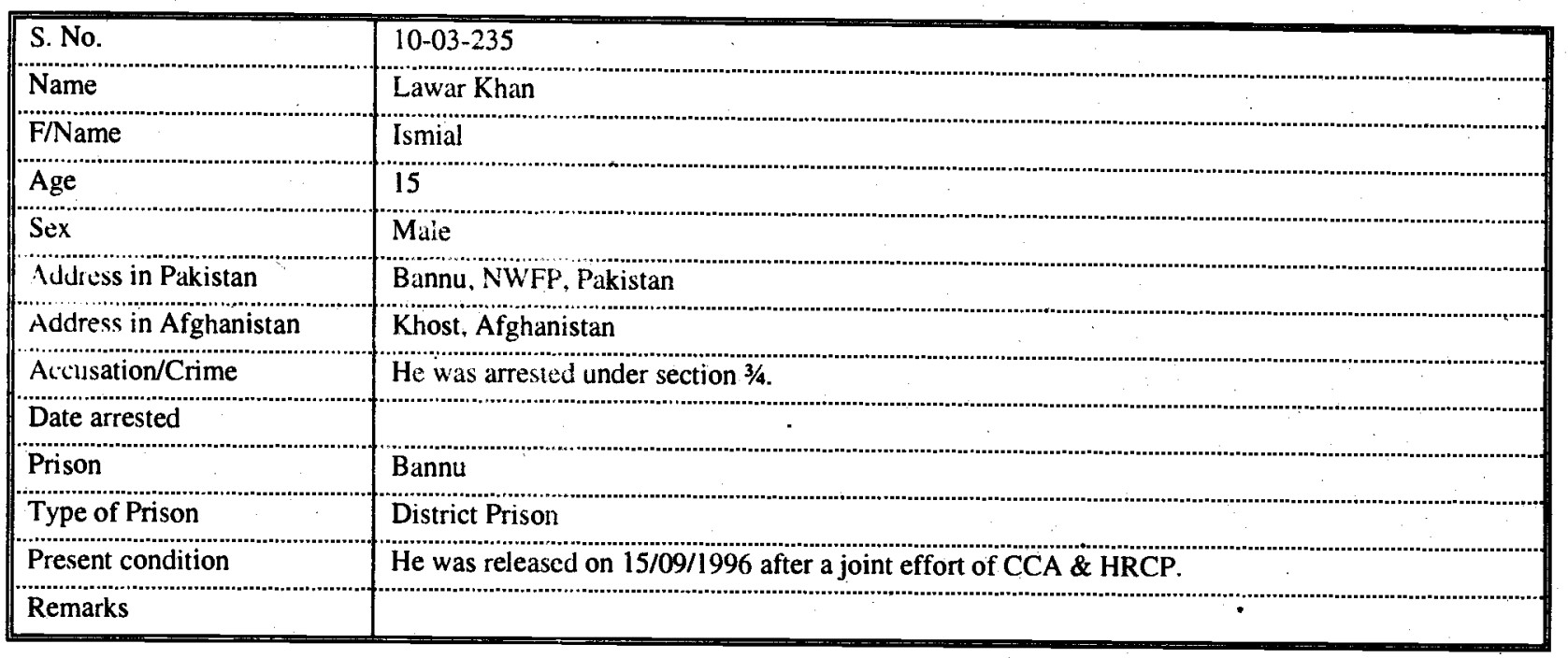

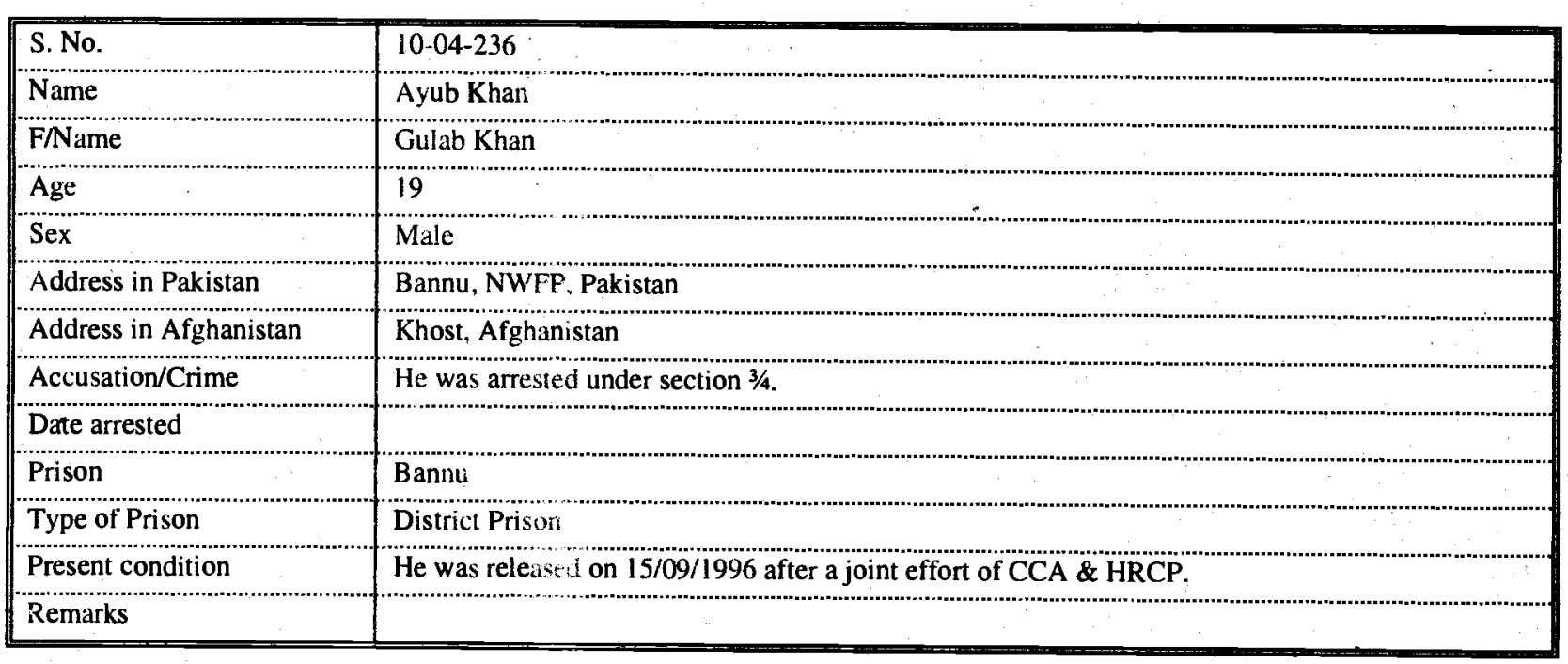




\begin{tabular}{|c|c|}
\hline S. No. & $10-05-237$ \\
\hline Name & Umar Khan \\
\hline F/Name & Mohammed Gul \\
\hline Age & 23 \\
\hline Sex & Male \\
\hline \multicolumn{2}{|c|}{ Address in Afghanistan } \\
\hline Accusation/Crime & He was arrested under section $107 / 151$. \\
\hline \multicolumn{2}{|l|}{ Date arrested } \\
\hline Prison & Bannu \\
\hline Type of Prison & District Prison \\
\hline
\end{tabular}

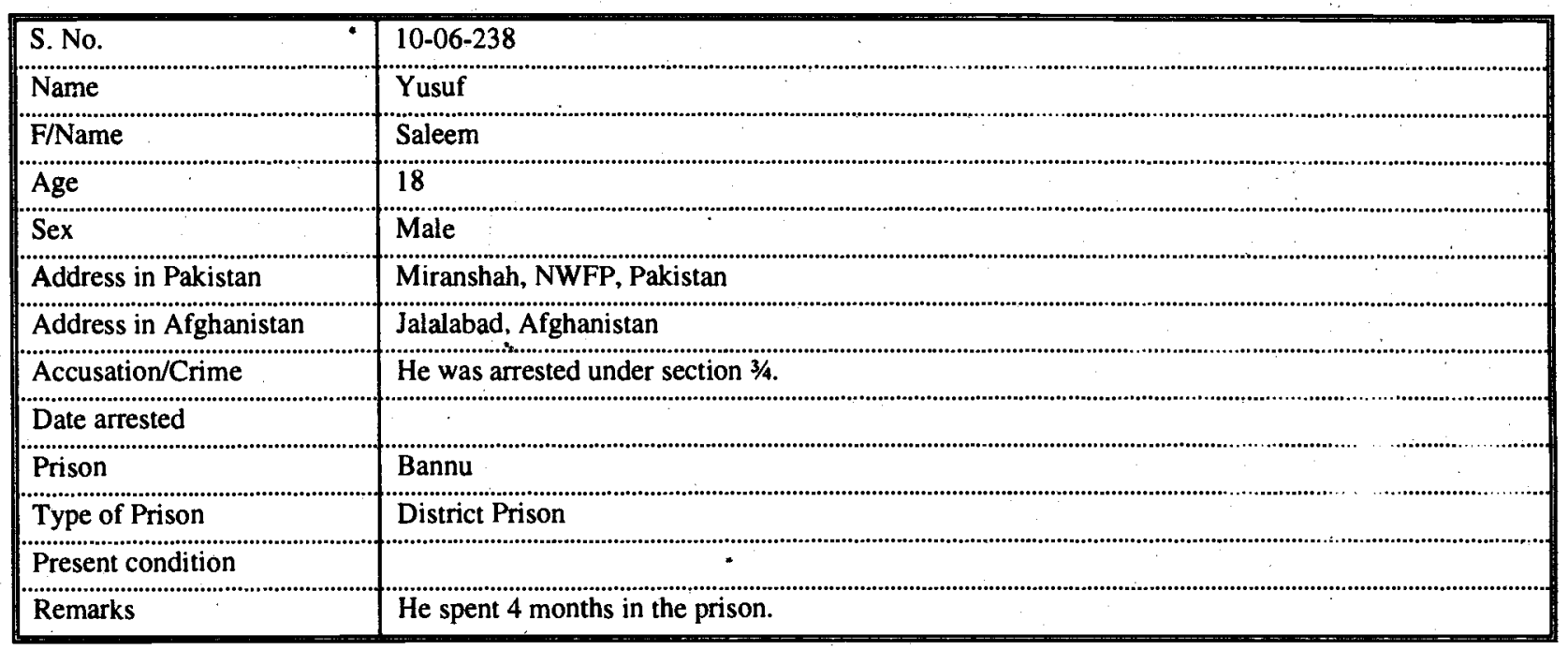

\begin{tabular}{|c|c|}
\hline S. No. & $10-07-239$ \\
\hline Name & Jahangir Khan \\
\hline F/Nanie & Mohammed Ayub \\
\hline Age & 31 \\
\hline Sex & Male \\
\hline Accusation/Crime & He was arrested under section 107 . \\
\hline Date arrested & 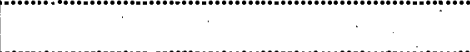 \\
\hline Prison & Bannu \\
\hline Type of Prison & District Prison \\
\hline
\end{tabular}




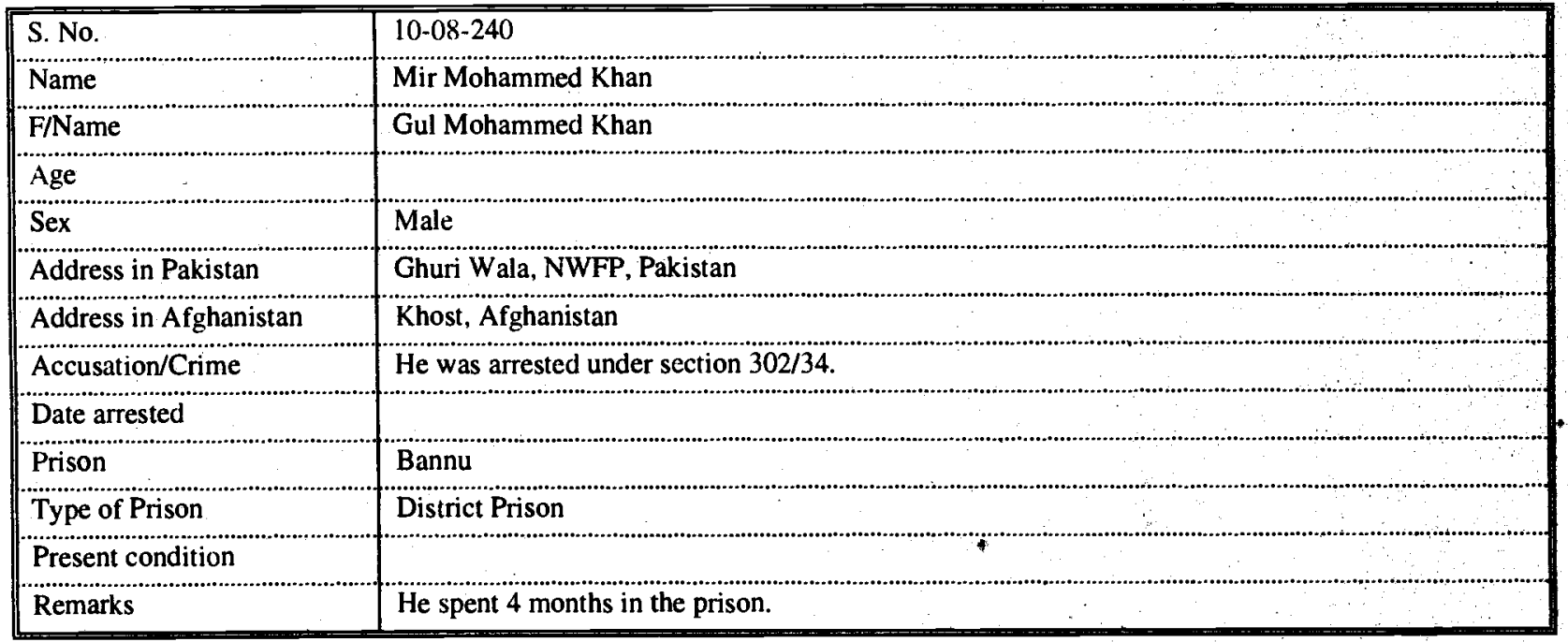

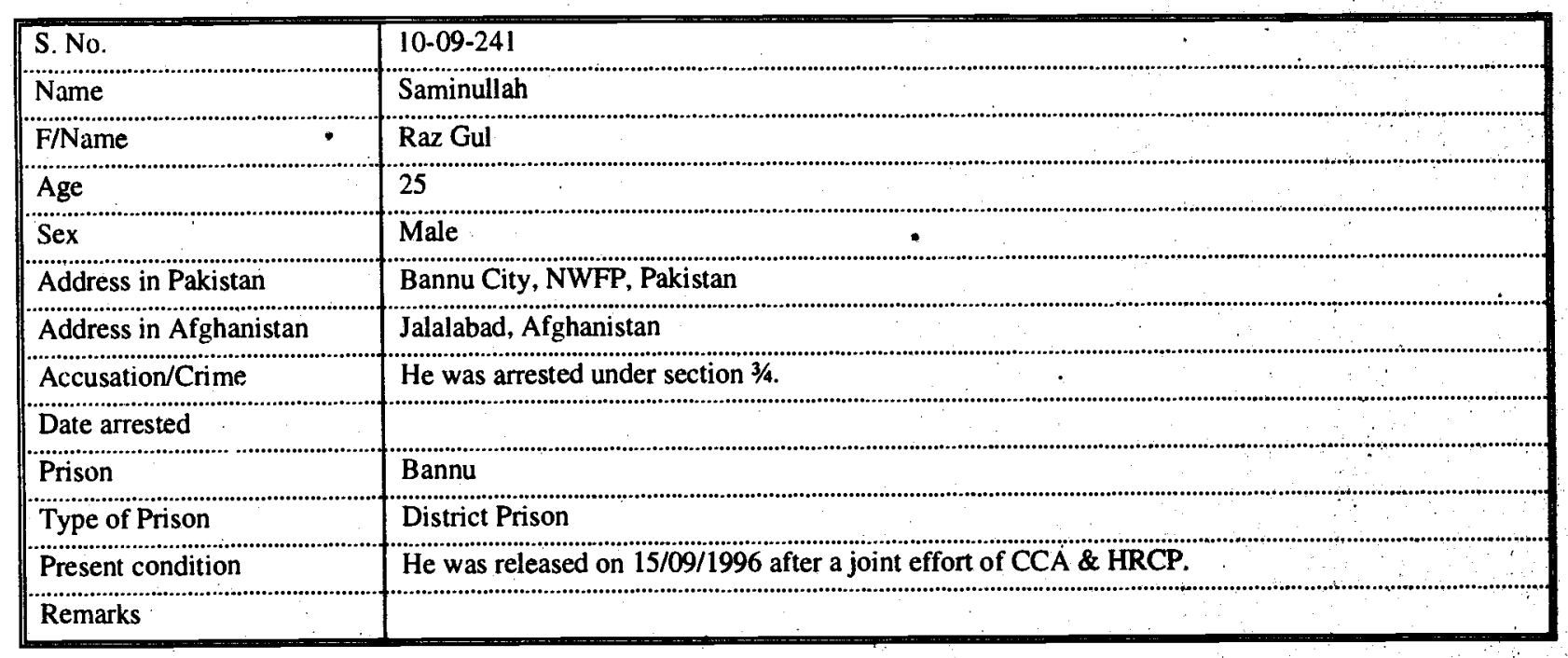

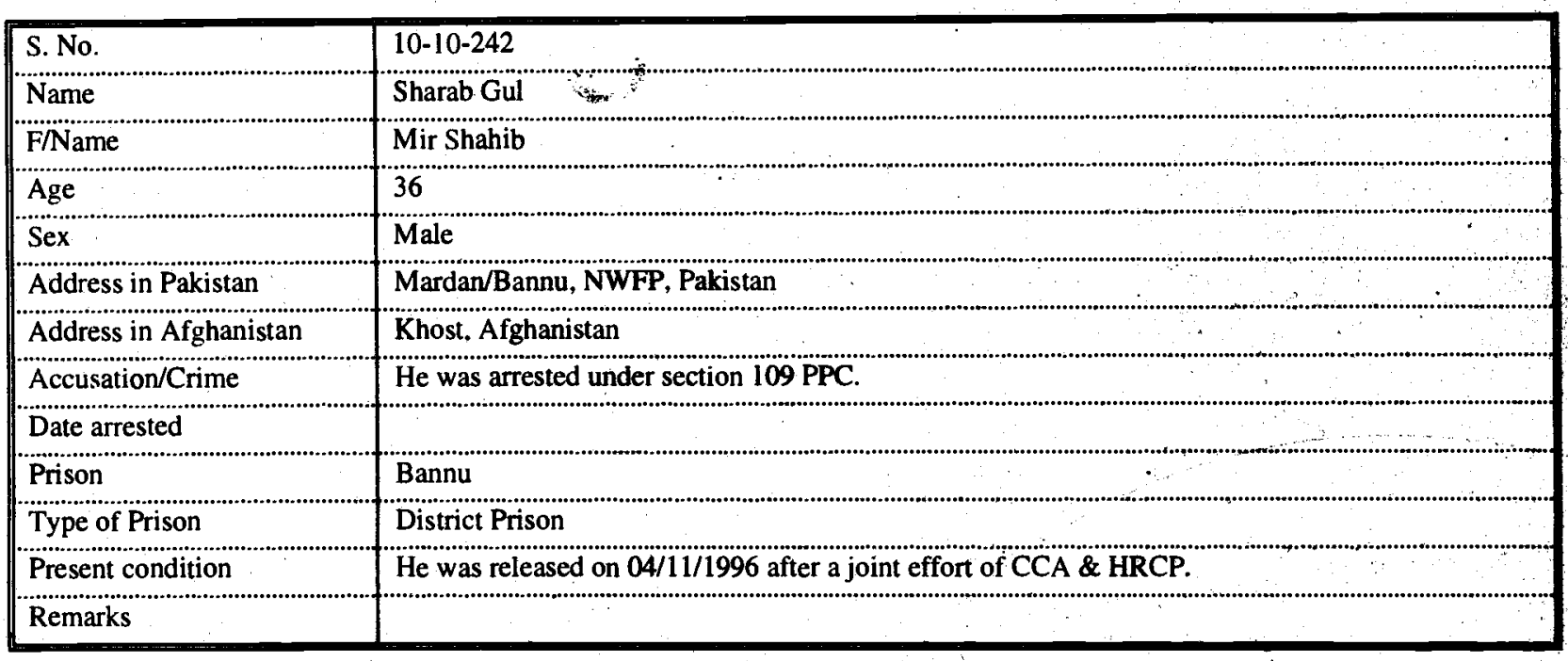




\begin{tabular}{|c|c|}
\hline S. No. & $10-11-243$ \\
\hline Name & Amir Ali Shah \\
\hline FiName & Gul Habib \\
\hline Age & 42 \\
\hline Sex & Male \\
\hline Address in Pakistan & Bannu City, NWFP, Pakistan \\
\hline Address in Afghanistan & Khost, Afghanistan \\
\hline Accusation/Crime & He was arrested under section $3 / 4$ \\
\hline Date arrested & \\
\hline Prison & Bannu \\
\hline Type of Prison & District Prison \\
\hline Present condition & He was released on 15/09/1996 after a joint effort of CCA \& HRCP. \\
\hline Remarks & ... \\
\hline
\end{tabular}

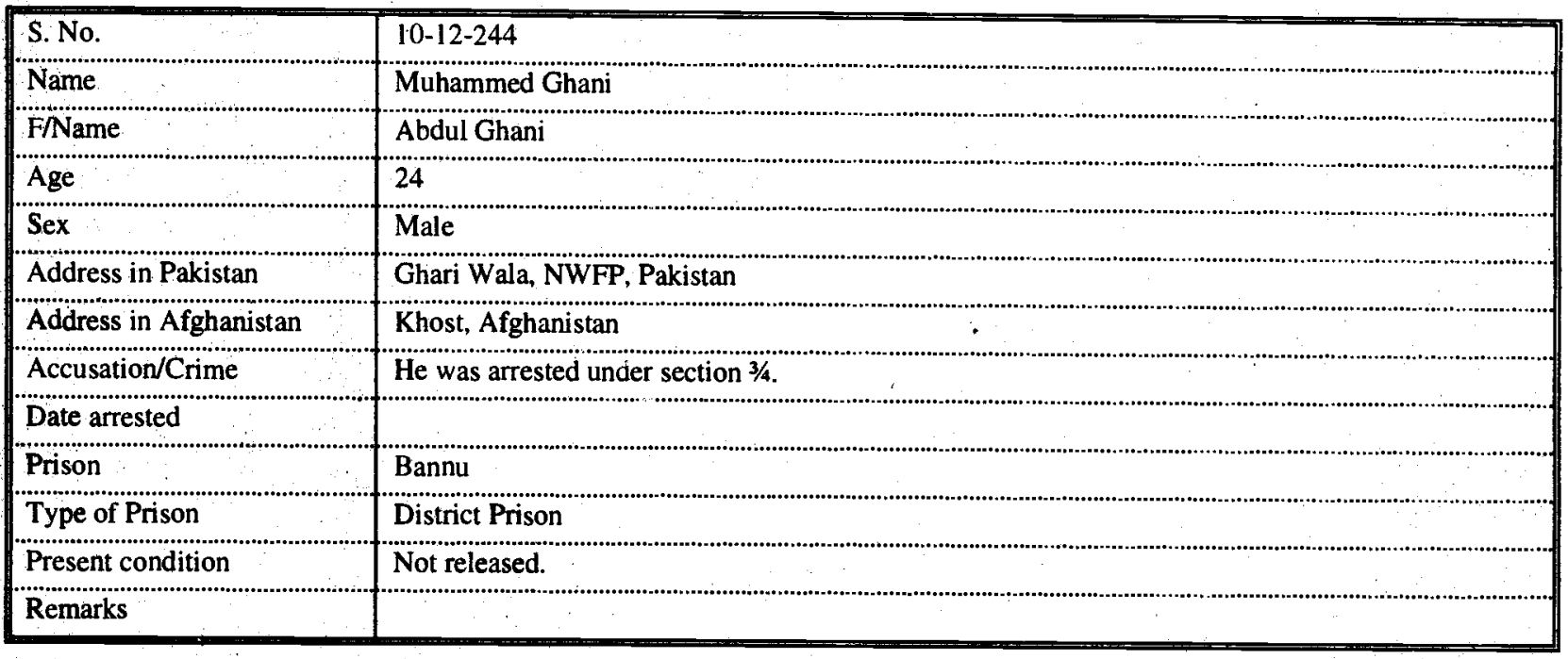

\begin{tabular}{|c|c|}
\hline S. No. & $10-13-245$ \\
\hline Name & Khairullah \\
\hline F/Name & Arabuddin \\
\hline Age & 31 \\
\hline Sex & Male \\
\hline Address in Pakistan & Pezo, NWFP, Pakistan \\
\hline Address in Afghanistan & Jalalabad, Afghanistan \\
\hline Accusation/Crime & He was arrested under section $3 / 4$. \\
\hline \multicolumn{2}{|l|}{ Date arrested } \\
\hline Prison & Bannu \\
\hline Type of Prison & District Prison \\
\hline Present condition & 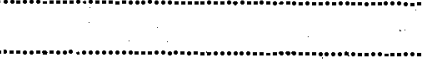 \\
\hline Remarks & He spent 7 months in the prison. \\
\hline
\end{tabular}




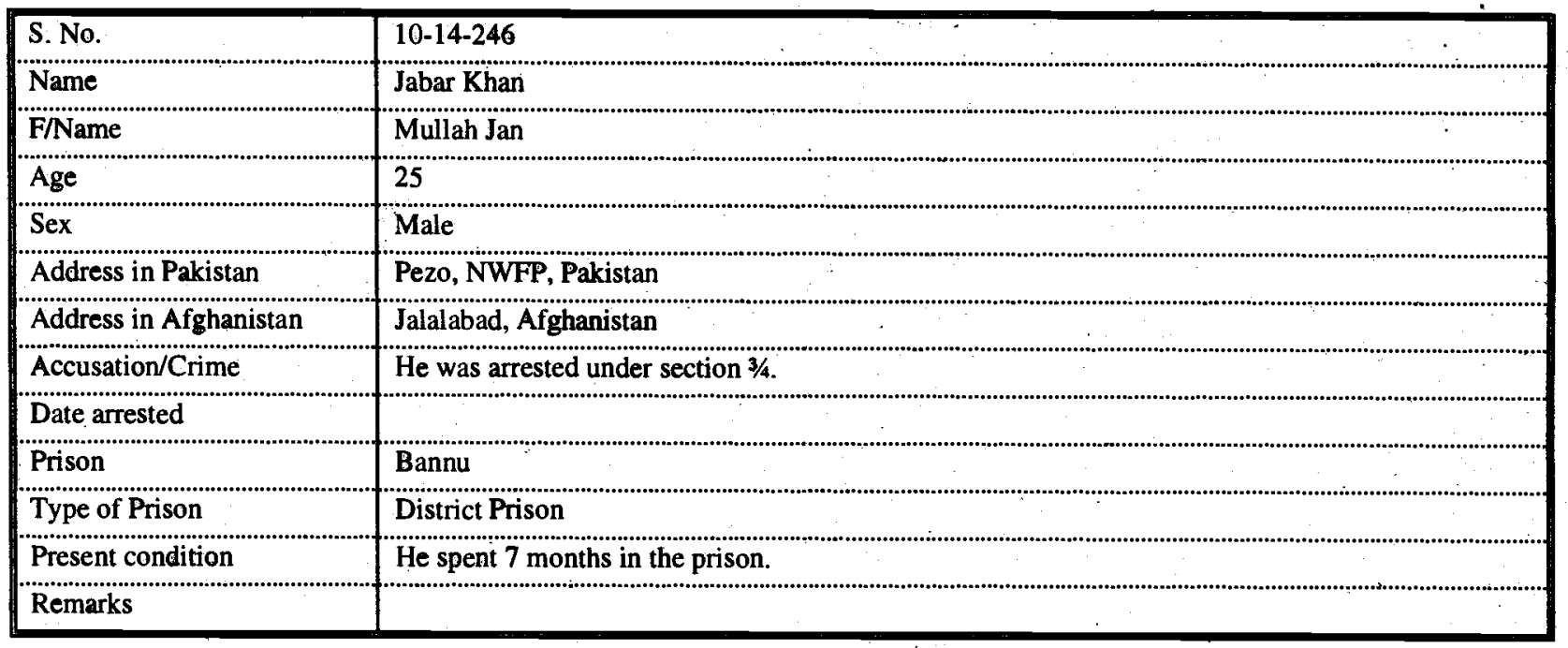

\begin{tabular}{|c|c|}
\hline S. No. & $10-15-247$ \\
\hline F/Name & Sardar Jan \\
\hline Age & 30 \\
\hline Sex & Male. \\
\hline Accusation/Crime & He was arrested under section 109 . \\
\hline \multicolumn{2}{|l|}{ Date arrested } \\
\hline Prison & Bannu \\
\hline
\end{tabular}

\begin{tabular}{|c|c|}
\hline S. No. & $10-16-248$ \\
\hline Name & Kamandan \\
\hline F/Name & Mukhtiyar \\
\hline Age & . \\
\hline Sex & Male \\
\hline Accusation/Crime & He was arrested under section $107 / 109 / 25$. \\
\hline Date arrested & . \\
\hline Prison & Bannu \\
\hline Type of Prison & District Prison \\
\hline
\end{tabular}




\begin{tabular}{|c|c|}
\hline S. No. & $10-17-249$ \\
\hline Name & Bilqees \\
\hline F/Name & Jamil \\
\hline Age & 16 \\
\hline Sex & Female \\
\hline Address in Pakistan & Takht Nasrati, NWFP, Pakistan \\
\hline Address in Afghanistan & \\
\hline Accusation/Crime & He was arrested under section $5 / 10 / 11$. \\
\hline Date arrested & \\
\hline Prison & Bannu \\
\hline Type of Prison & District Prison \\
\hline Present condition & He was released on 04/11/1996 after a joint effort of CCA \& HRCP. \\
\hline Remarks & \\
\hline
\end{tabular}




\section{Central Prison Dara Ismial Khan}

\begin{tabular}{|c|c|}
\hline S. No. & $11-01-250$ \\
\hline Name & Badshah Hussain \\
\hline F/Name & Sayyed Hussain \\
\hline Age & 35 \\
\hline Sex & Male \\
\hline Address in Pakistan & Mehran Shah, NWFP, Pakistan \\
\hline Address in Afghanistan & Jalalabad, Afghanistan \\
\hline Accusation/Crime & He was arrested under section 11 FCR. \\
\hline Date arrested & \\
\hline Prison & DI Khan \\
\hline Type of Prison & Central Prison \\
\hline Present condition & Not released. \\
\hline Remarks & \\
\hline
\end{tabular}




\begin{tabular}{|l|l|l|}
\hline S. No. & $11-02-251$ \\
\hline Name & Badshah & Sayyed Hussain \\
\hline F/Name & 30 \\
\hline Age & Male \\
\hline Sex & Behran Shah, NWFP, Pakistan \\
\hline Address in Pakistan & Jalalabad, Afghanistan \\
\hline Address in Afghanistan & He was arrested under section 11 FCR. \\
\hline Accusation/Crime & DI Khan \\
\hline Date arrested & Central Prison \\
\hline Prison & \\
\hline Type of Prison & He spent 2 and a half year in the prison. \\
\hline Present condition & \\
\hline Remarks & &
\end{tabular}

\begin{tabular}{|l|l}
\hline S. No. & $11-03-252$ \\
\hline Name & Abduseed \\
\hline Foname & Dilbar Jan \\
\hline Age & 40 \\
\hline Sex & Male \\
\hline Address in Pakistan & Mehran Shah, NWFP, Pakistan \\
\hline Address in Afghanistan & Jalalabad, Afghanistan \\
\hline Accusation/Crime & He was arrested under section 11 FCR. \\
\hline Date arrested & \\
\hline Prison & DI Khan \\
\hline Type of Prison & Central Prison \\
\hline Present condition & He spent 3 and a half years in the prison. \\
\hline Remarks &
\end{tabular}

\begin{tabular}{|l|l|l|}
\hline S. No. & $11-04-253$ \\
\hline Name & Abdullah \\
\hline F/Name & Ziarat \\
\hline Age & 39 \\
\hline Sex & Male \\
\hline Address in Pakistan & Mehran Shah, NWFP, Pakistan \\
\hline Address in Afghanistan & Jalalabad, Afghanistan \\
\hline Accusation/Crime & He was arrested under section 11 FCR. \\
\hline Date arrested & DI Khan \\
\hline Prison & Central Prison \\
\hline Type of Prison & \\
\hline Present condition & He spent two and a half year in the prison. \\
\hline Remarks &
\end{tabular}




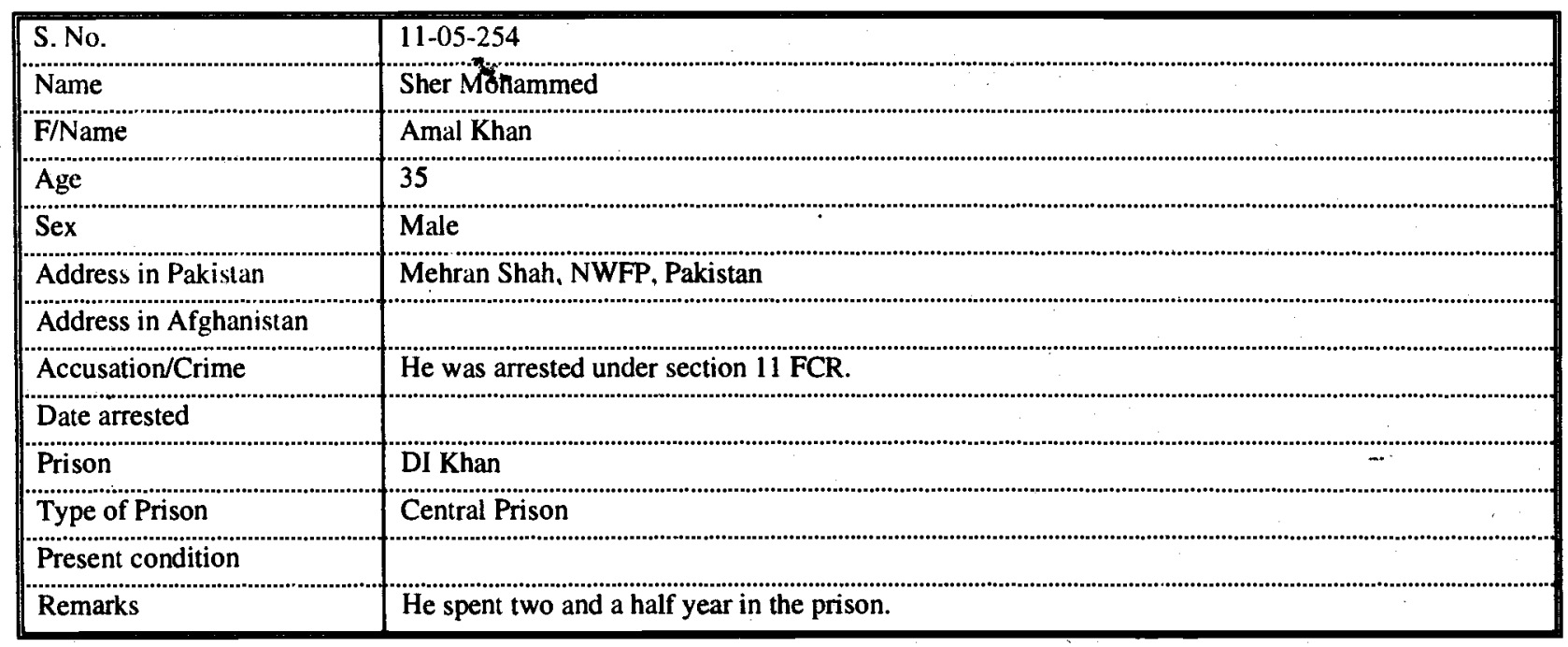

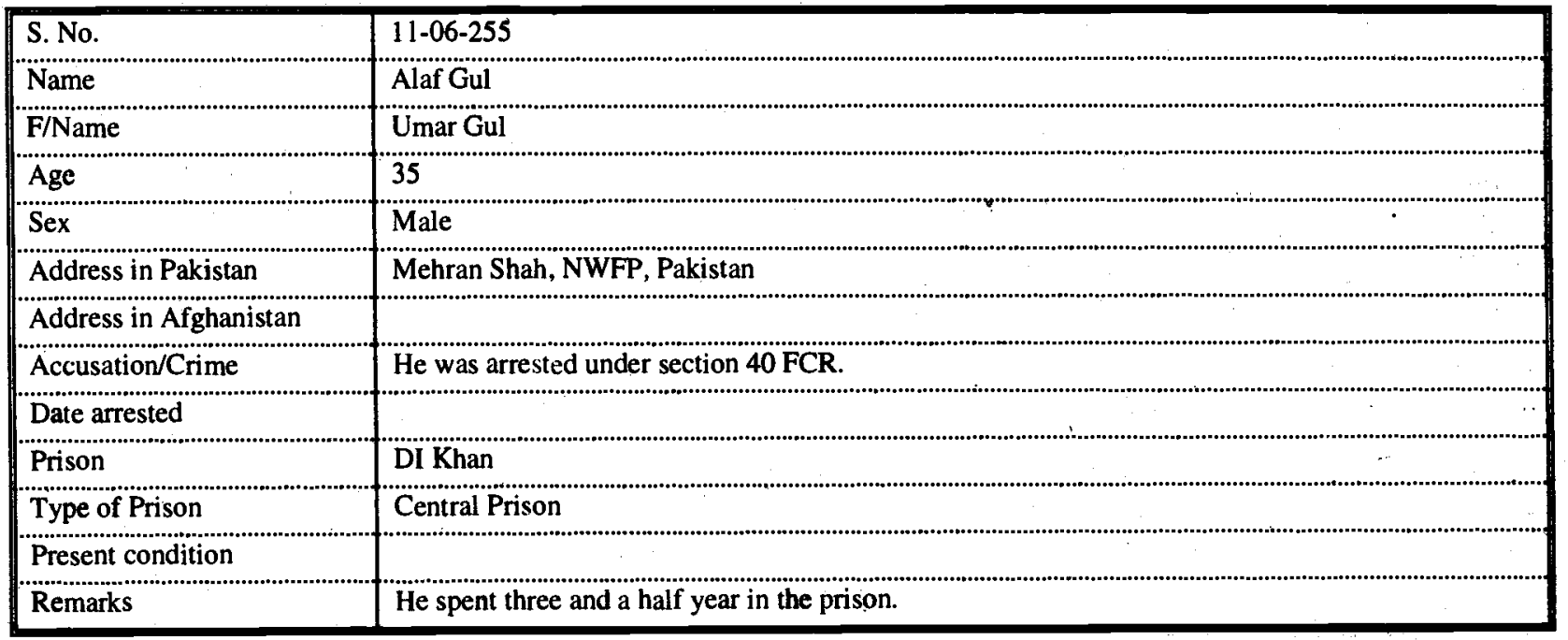

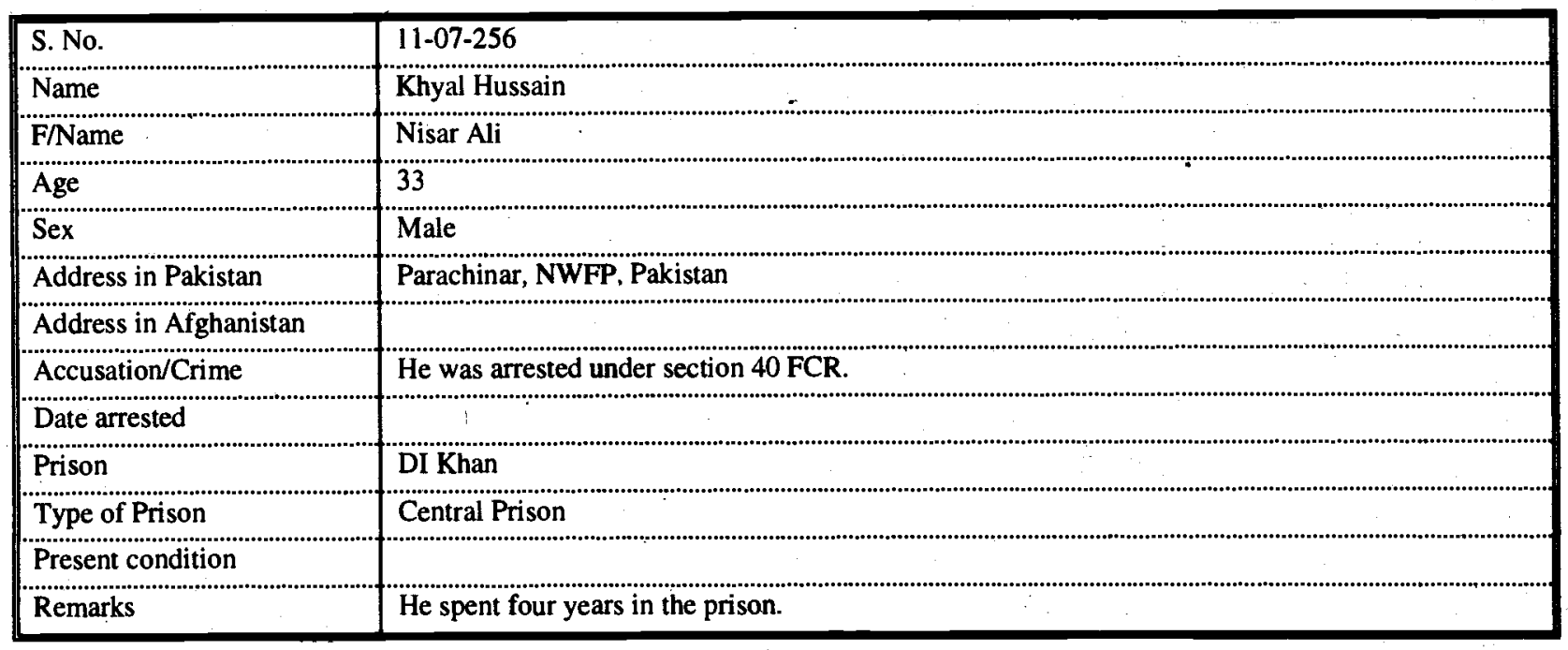




\begin{tabular}{|c|c|}
\hline S. No. & $11-08-257$ \\
\hline Name & Khan Mohammed \\
\hline F/Name & Meranshah \\
\hline Age & 35 \\
\hline Sex & Male \\
\hline \multicolumn{2}{|l|}{ Accusation/Crime } \\
\hline \multicolumn{2}{|l|}{ Date arrested } \\
\hline Prison & DI Khan \\
\hline Type of Prison & Central Prison \\
\hline
\end{tabular}

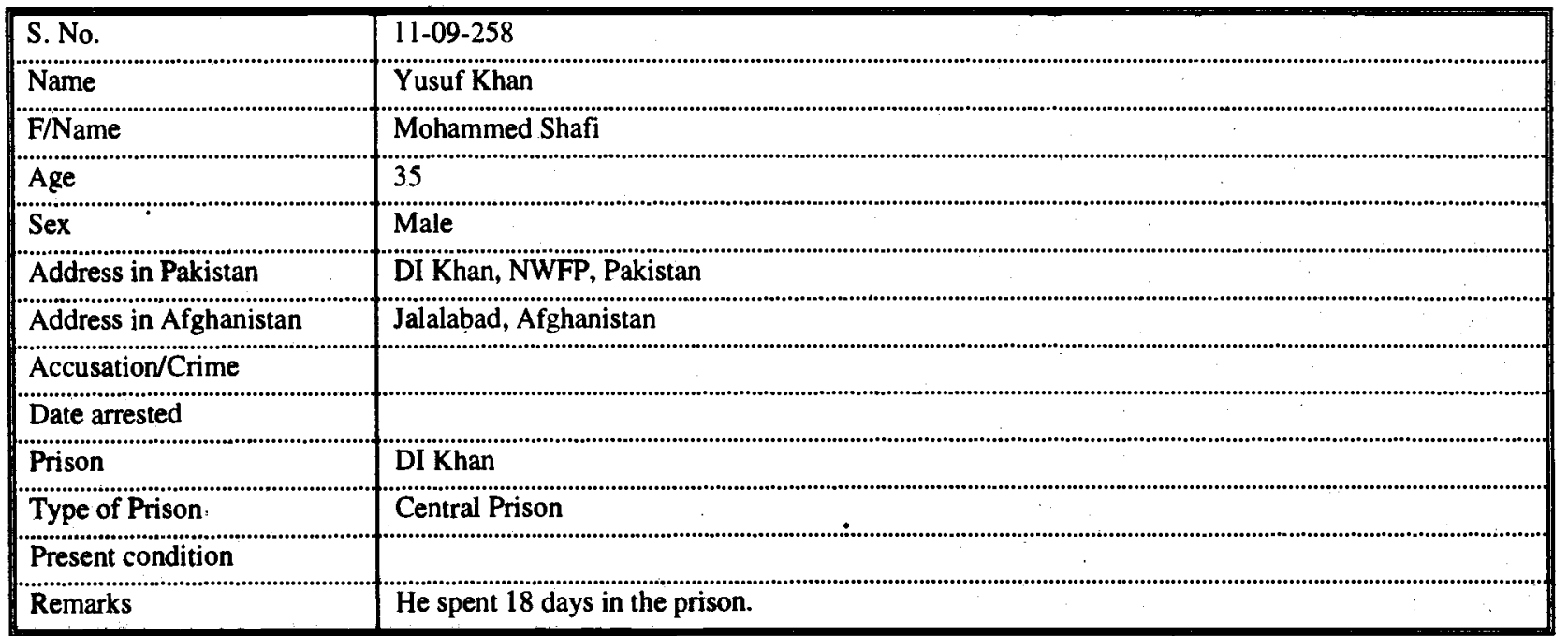

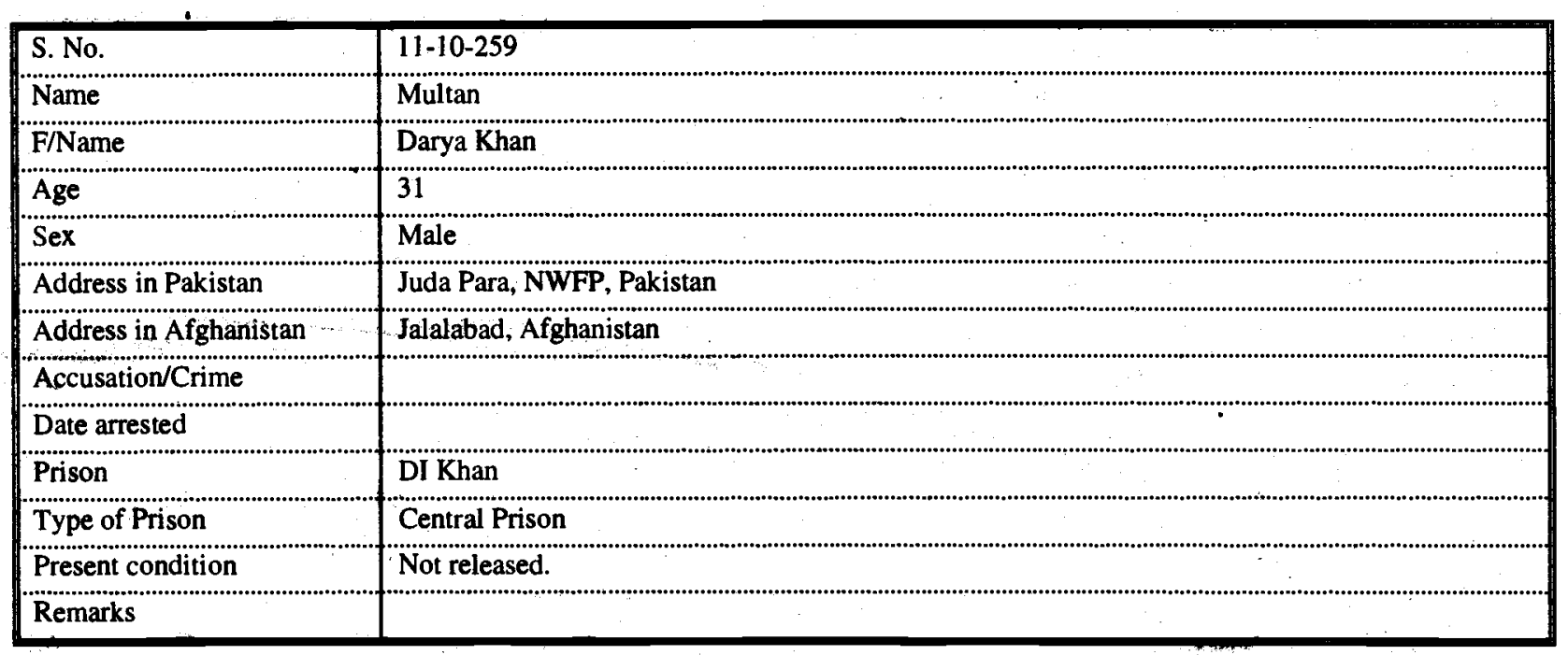




\begin{tabular}{|c|c|}
\hline S. No. & $11-11-260$ \\
\hline Name & Sahib Jan \\
\hline F/Name & Esa Khan \\
\hline \multicolumn{2}{|l|}{ Age } \\
\hline Sex & Male \\
\hline Address in Pakistan & D I Khan. NWFP, Pakistan \\
\hline Address in Afghanistan & Khost. Afghanistan \\
\hline Accusation/Crime & He was arrested under section $107 / 151$. \\
\hline \multicolumn{2}{|l|}{ Date arrested } \\
\hline Prison & DI Khan \\
\hline Type of Prison & Central Prison \\
\hline \multicolumn{2}{|l|}{ Present condition } \\
\hline Remarks & He spent 3 months in the prison. \\
\hline
\end{tabular}

\begin{tabular}{|c|c|}
\hline S. No. & $11-12-261$ \\
\hline Name & Shawkat \\
\hline F/Name & Wali Mohammed \\
\hline Age & 30 \\
\hline Sex & Male \\
\hline Address in Pakistan & Cantt, Bannu, NWFP, Pakistan \\
\hline Address in Afghanistan & \\
\hline Accusation/Crime & He was arrested under section $107 / 151$. \\
\hline Date arrested & \\
\hline Prison & DI Khan \\
\hline Type of Prison & Central Prison \\
\hline Present condition & Not released. \\
\hline Remarks & \\
\hline
\end{tabular}

\begin{tabular}{|c|c|}
\hline S. No. & $11-13-262$ \\
\hline Name & Qismetullah" \\
\hline F/Name & Kafayatullah \\
\hline \multicolumn{2}{|l|}{ Age } \\
\hline Sex & Male \\
\hline Address in Pakistan & D I Khan City, NWFP, Pakistan \\
\hline \multicolumn{2}{|c|}{ Address in Afghanistan } \\
\hline Accusation/Crime & He was arrested under section $107 / 109 / 151$. \\
\hline \multicolumn{2}{|l|}{ Date arrested } \\
\hline Prison & DI Khan \\
\hline Type of Prison & Central Prison \\
\hline \multicolumn{2}{|l|}{ Present condition } \\
\hline Remarks & He spent 3 months in the prison. \\
\hline
\end{tabular}




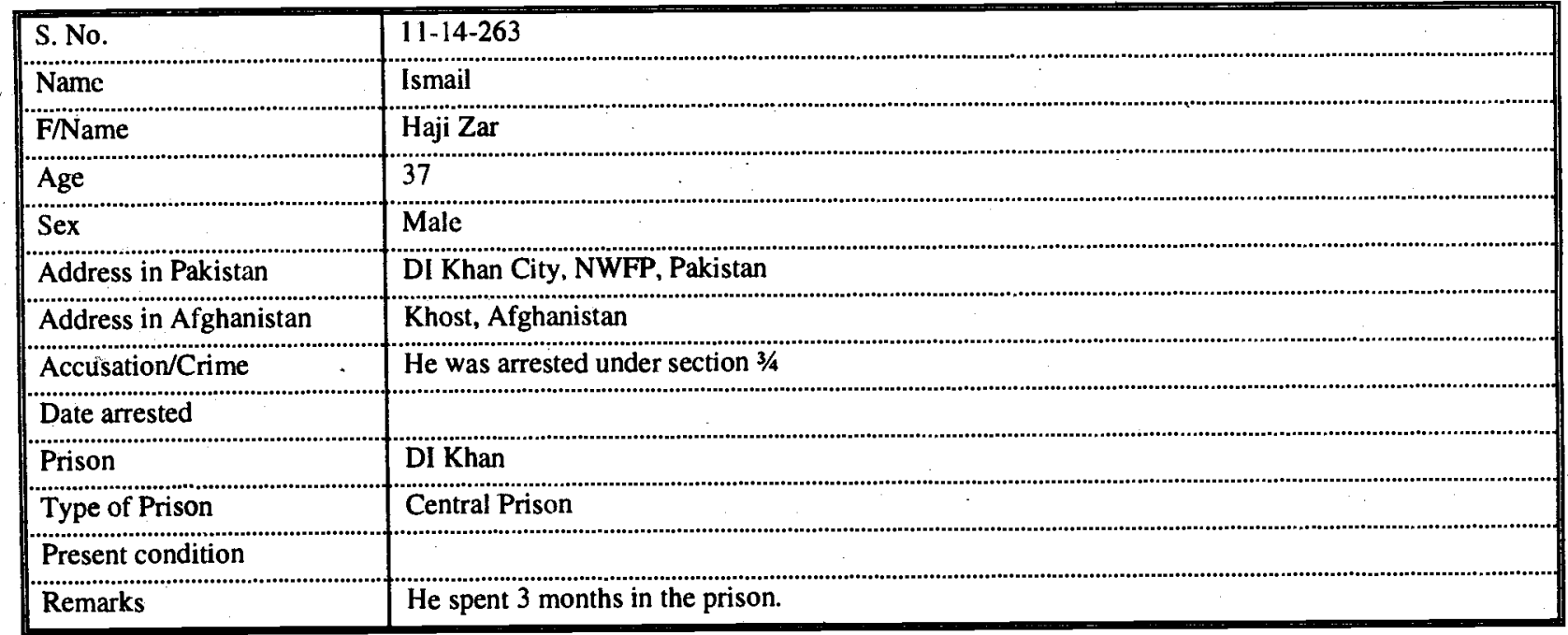

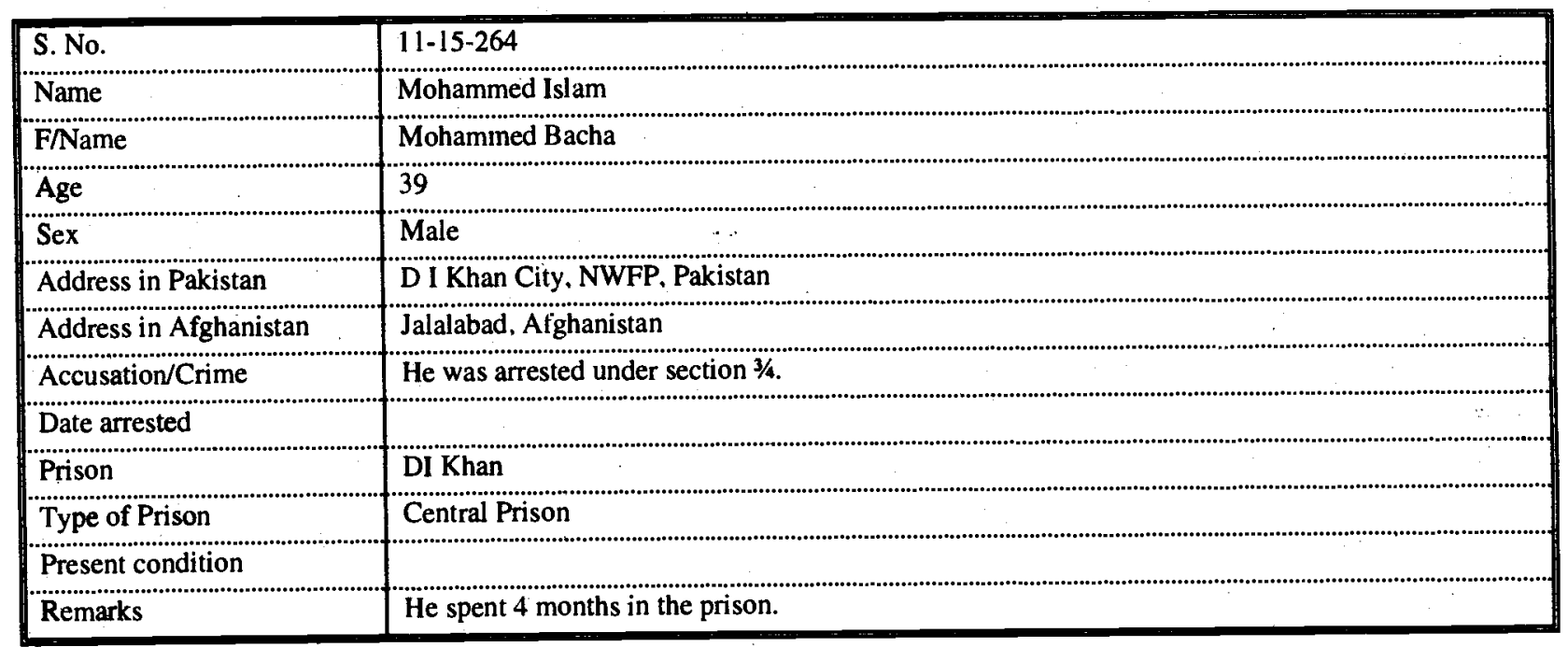




\section{Ludicial Lockup Bajaur}

\begin{tabular}{|c|c|}
\hline S. No. & $12-01-265$ \\
\hline Name & Lal Gul \\
\hline F/Name & Mir Gul \\
\hline Age & 35 \\
\hline Sex & Male \\
\hline Address in Pakistan & Maingawere Swat, NWFP, Pakistan \\
\hline Address in Afghanistan & \\
\hline Accusation/Crime & He was arrested under section $107 / 109$. \\
\hline Date arrested & \\
\hline Prison & Bajaur \\
\hline Type of Prison & District Prison \\
\hline Present condition & Not released. \\
\hline Remarks & \\
\hline
\end{tabular}




\begin{tabular}{|c|c|}
\hline S. No. & $12-02-266$ \\
\hline Name & Painda Khan \\
\hline F/Name & Mohammed Farooq \\
\hline $\mathrm{Age}$ & 25 \\
\hline Sex & Male \\
\hline Address in Pakistan & Yusuf Abad, Camp, NWFP, Pakistan \\
\hline Address in Afghanistan & ( \\
\hline 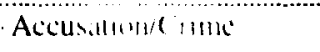 & He was arrested under section $5 / 10 / 11, \ldots$ \\
\hline \multicolumn{2}{|l|}{ Date arrested } \\
\hline Prison & Bajaur \\
\hline Type of Prison & District Prison \\
\hline Present condition & Not released. \\
\hline Remarks & \\
\hline
\end{tabular}

\begin{tabular}{|c|c|}
\hline S. No. & $12-03-267$ \\
\hline Name & Haider Zaman \\
\hline F/Name & Gul Bar \\
\hline Age & 18 \\
\hline Sex & Male \\
\hline Accusation/Crime & He was arrested under section $506 / 107$. \\
\hline Date arrested & ? \\
\hline Prison & Bajaur \\
\hline Type of Prison & District Prison \\
\hline
\end{tabular}

\begin{tabular}{|c|c|}
\hline S. No. & $12-04-268$ \\
\hline F/Name & Radi Gul \\
\hline Age & 30 \\
\hline Sex & Male \\
\hline Accusation/Crime & He was arrested under section $420 / 419$. \\
\hline \multicolumn{2}{|l|}{ Date arrested } \\
\hline Prison & Bajaur \\
\hline Type of Prison & District Prison \\
\hline
\end{tabular}




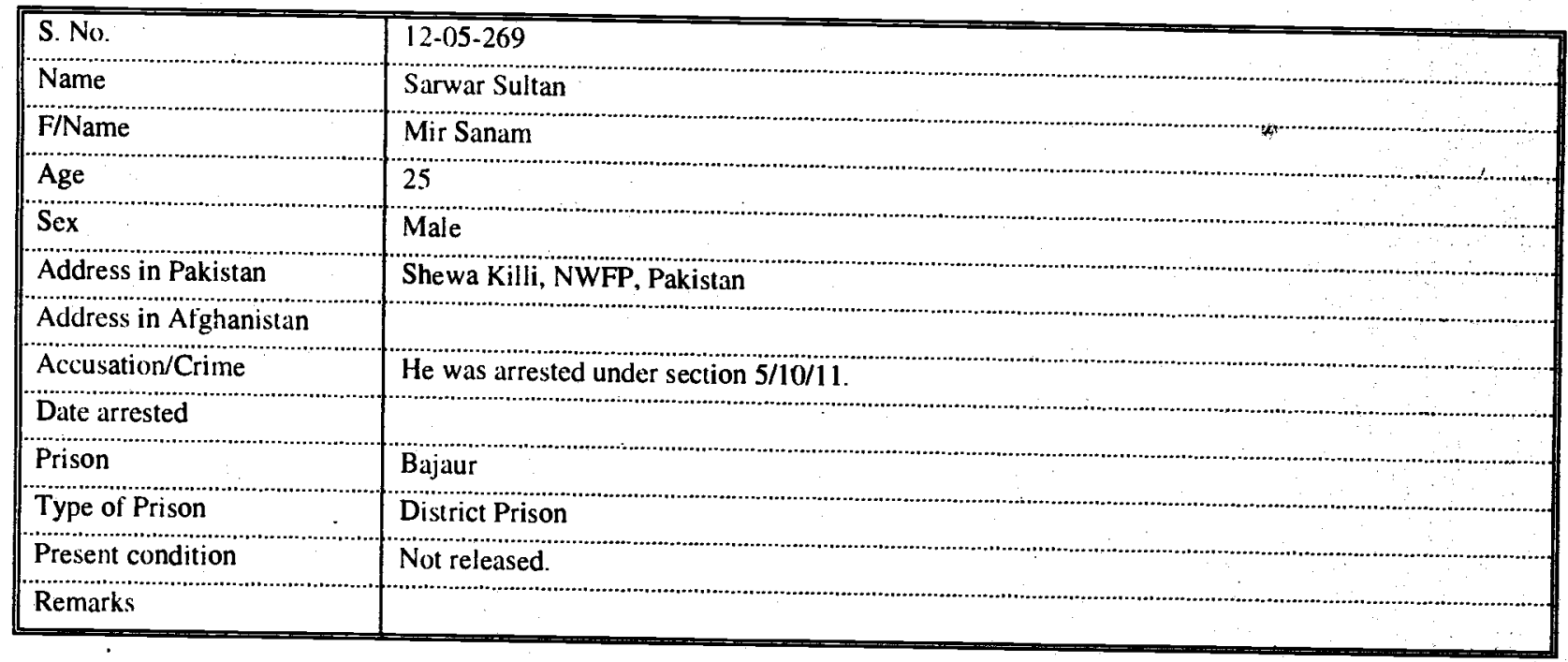

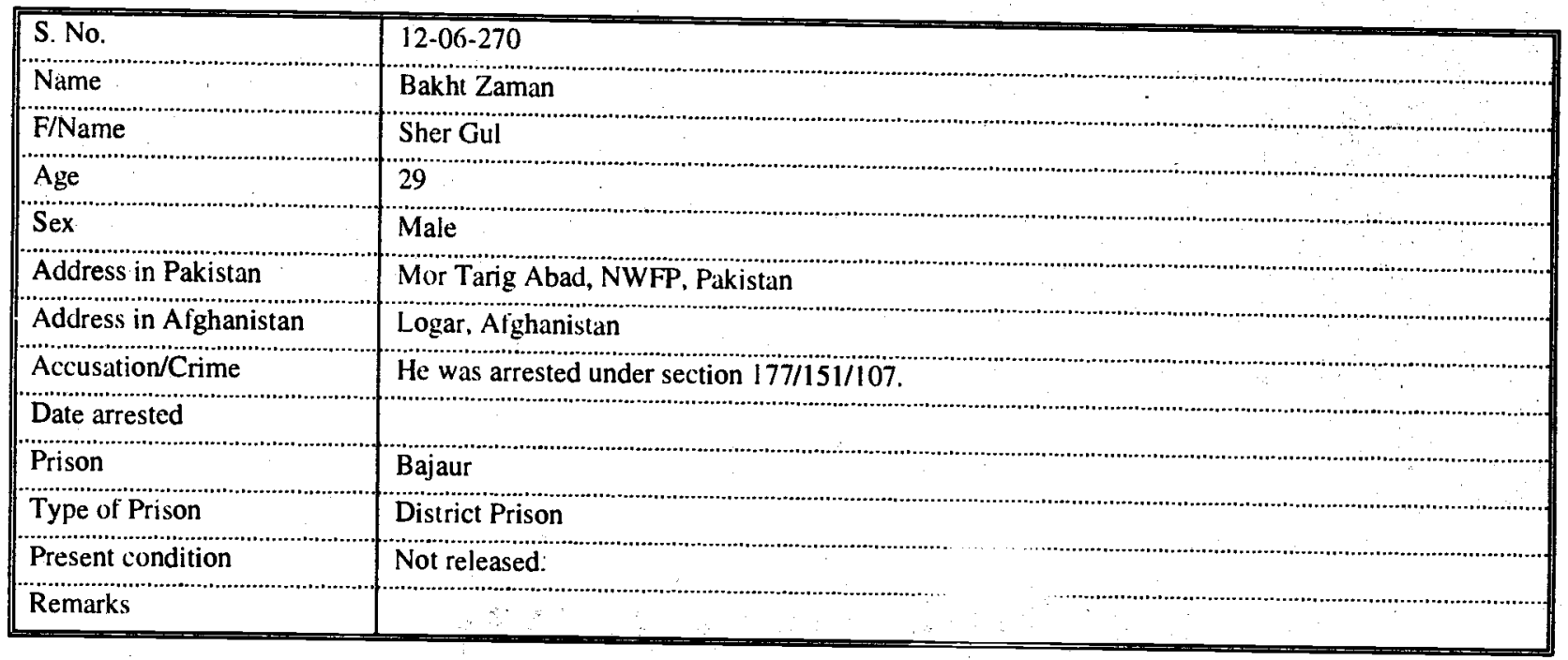

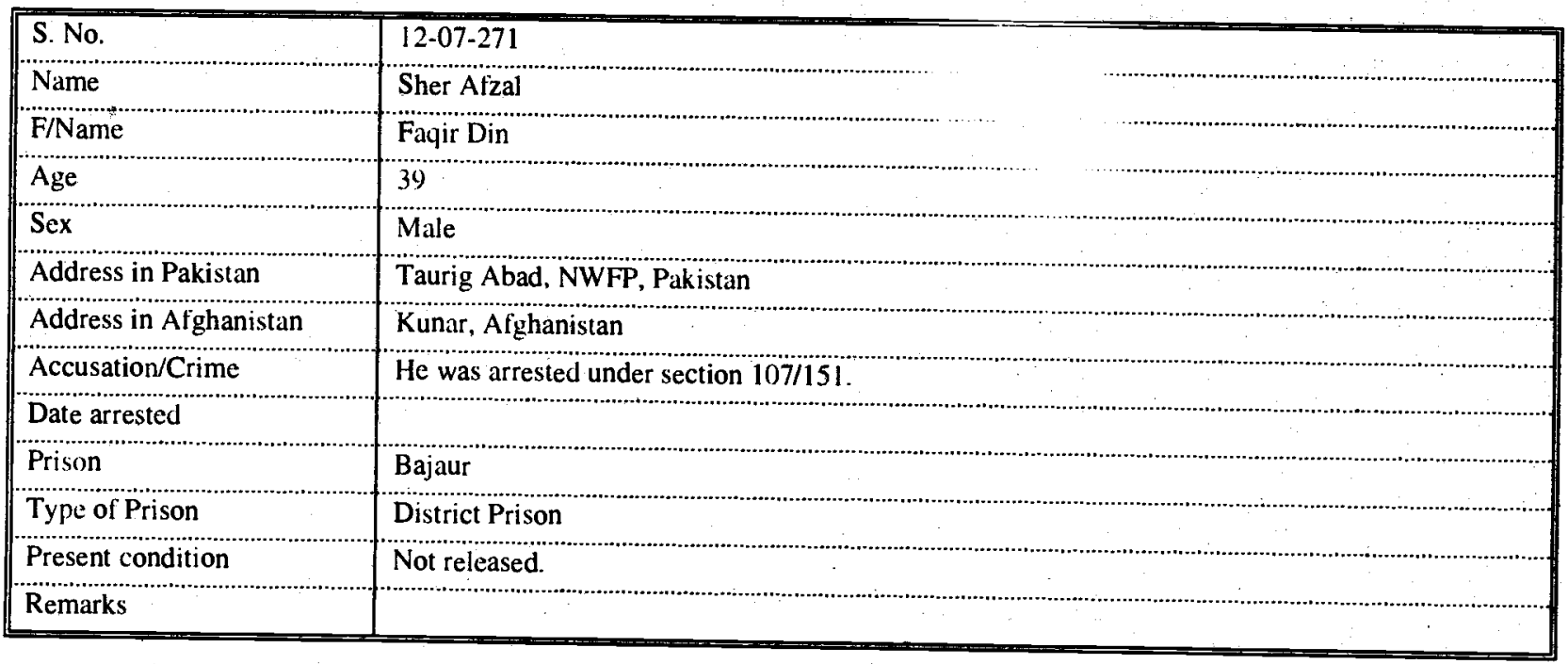




\section{Iudicial Lockup Dir (upper)}

\begin{tabular}{|l|l|l|}
\hline S. No. & $13-01-272$ \\
\hline Name & Mohammed Rahim & Rahimullah \\
\hline F/Name & 35 & Male \\
\hline Age & Mayar Camp, NWFP, Pakistan \\
\hline Sex & Kunar, Afghanistan \\
\hline Address in Pakistan & He was arrested under section 107/151. \\
\hline Address in Afghanistan & Dir (upper) \\
\hline Accusation/Crime & Sub Jail \\
\hline Date arrested & \begin{tabular}{l} 
Not released \\
\hline Prison
\end{tabular} \\
\hline Type of Prison & \\
\hline Present condition & \\
\hline Remarks &
\end{tabular}




\begin{tabular}{|c|c|}
\hline S. No. & $13-02-273$ \\
\hline Name & Hamidur Rahman \\
\hline F/Name & Fatil Urrahman \\
\hline Age & 40 \\
\hline Sex & Male \\
\hline Address in Pakistan & Yusuf Abad Camp, NWFP, Pakistan \\
\hline Address in Afghanistan & Kunar, Afghanistan \\
\hline Accusation/Crime & He was arrested under section 379/107/109. \\
\hline \multicolumn{2}{|l|}{ Date arrested } \\
\hline Prison & Dir (upper) \\
\hline Type of Prison & Sub Jail \\
\hline Present condition & Not released. \\
\hline Remarks & \\
\hline
\end{tabular}

\begin{tabular}{|c|c|}
\hline S. No. & $13-03-274$ \\
\hline Name & Abdul Haq \\
\hline F/Name & Rahmat Sayyed \\
\hline Age & 25 \\
\hline Sex & Male \\
\hline Address in Pakistan & Toor Camp, NWFP, Pakistan \\
\hline Address in Afghanistan & Kunar, Afghanistan \\
\hline Accusation/Crime & He was arrested under section 109/107/151. \\
\hline \multicolumn{2}{|l|}{ Date arrested } \\
\hline Prison & Dir (upper) \\
\hline Type of Prison & Sub Jail \\
\hline Present condition & Not released. \\
\hline Remarks & \\
\hline
\end{tabular}

\begin{tabular}{|c|c|c|}
\hline S. No. & $13-04-275$ & \\
\hline Name & Gul Bacha & \\
\hline F/Name & Nazar & \\
\hline Age & 30 & \\
\hline Sex & Male & \\
\hline Address in Pakistan & Sairai, NWFP, Pakistan & \\
\hline Address in Afghanistan & Jalalabad, Afghanistan & $\rightarrow$ \\
\hline Accusation/Crime & He was arrested under section $151 / 107 / 109$. & \\
\hline \multicolumn{3}{|l|}{ Date arrested } \\
\hline Prison & Dir (upper) & \\
\hline Type of Prison & Sub Jail & \\
\hline Present condition & Not released. & \\
\hline Remarks & : & \\
\hline
\end{tabular}




\begin{tabular}{|c|c|}
\hline S. No. & $13-05-276$ \\
\hline Name & Hamidullah \\
\hline F/Name & Rahmat Jan \\
\hline Age & 35 \\
\hline Sex & Male \\
\hline Address in Pakistan & Lal Qila, NWFP, Pakistan \\
\hline Address in Afghanistan & Jalalabad, Afghanistan \\
\hline Accusation/Crime & He was arrested under section 107/151. \\
\hline \multicolumn{2}{|l|}{ Date arrested } \\
\hline Prison & Dir (upper) \\
\hline Type of Prison & Sub Jail \\
\hline Present condition & Not released. \\
\hline Remarks & \\
\hline
\end{tabular}

\begin{tabular}{|c|c|}
\hline S. No. & $13-06-277$ \\
\hline Name & Abdullah \\
\hline F/Name & Amarullah \\
\hline Age & 28 \\
\hline Sex & Make \\
\hline Address in Pakistan & Dir Camp, NWFP, Pakistan \\
\hline \multicolumn{2}{|l|}{ Address in Afghanistan } \\
\hline Accusation/Crime & He was arrested under section $302 / 188$. \\
\hline \multicolumn{2}{|l|}{ Date arrested } \\
\hline Prison & Dir (upper) \\
\hline Type of Prison & Sub Jail \\
\hline Present condition & Not released. \\
\hline Remarks & \\
\hline
\end{tabular}

\begin{tabular}{|c|c|}
\hline S. No. & $13-07-278$ \\
\hline Name & Bahadur Khan \\
\hline F/Name & Toor Khan \\
\hline Age & 28 \\
\hline Sex & Male \\
\hline Address in Pakistan & Toor Camp, NWFP, Pakistan \\
\hline \multicolumn{2}{|l|}{ Address in Afghanistan } \\
\hline Accusation/Crime & He was arrested under section $5 / 10 / 11$. \\
\hline \multicolumn{2}{|l|}{ Date arrested } \\
\hline Prison & Dir (upper) \\
\hline Type of Prison & Sub Jail \\
\hline Present condition & Not released. \\
\hline Remarks & \\
\hline
\end{tabular}




\begin{tabular}{|c|c|}
\hline S. No. & $13-08-279$ \\
\hline Name & Sayyed Karim \\
\hline F/Name & Sayyed Amin \\
\hline Age & 25 \\
\hline Sex & Male \\
\hline Address in Pakistan & Mayar Camp, NWFP, Pakistan \\
\hline \multicolumn{2}{|c|}{ Äddress 'n Af Granistan } \\
\hline Accusation/Crime & He was arrested under section 377 . \\
\hline \multicolumn{2}{|l|}{ Date arrested } \\
\hline Prison & Dir (upper) \\
\hline Type of Prison & Sub Jail \\
\hline Present condition & Not released. \\
\hline Remarks & \\
\hline
\end{tabular}

\begin{tabular}{|c|c|}
\hline S. No. & $13-09-280$ \\
\hline Name & Umar Badshah \\
\hline F/Name & Mohammed \\
\hline Age & 40 \\
\hline Sex & Male \\
\hline Address in Pakistan & Dir Camp, Samar Bagh, NWFP, Pakistan \\
\hline Address in Afghanistan & Jalalabad, Afghanistan \\
\hline Accusation/Crime & He was arrested under section 279 . \\
\hline \multicolumn{2}{|l|}{ Date arrested } \\
\hline Prison & Dir (upper) \\
\hline Type of Prison & Sub Jail \\
\hline Present condition & Not released. \\
\hline Remarks & \\
\hline
\end{tabular}

\begin{tabular}{|c|c|}
\hline S. No. & $13-10-281$ \\
\hline Name & Subhanullah" \\
\hline F/Name & Shamandoz \\
\hline Age & 25 \\
\hline Sex & Male \\
\hline Address in Pakistan & Baramol Dir Camp, NWFP, Pakistan \\
\hline \multicolumn{2}{|c|}{ Address in Afghanistan } \\
\hline Accusation/Crime & He was arrested under section $5 / 10 / 11$. \\
\hline \multicolumn{2}{|l|}{ Date arrested } \\
\hline Prison & Dir (upper) \\
\hline Type of Prison & Sub Jail \\
\hline Present condition & Not released. \\
\hline Remarks & \\
\hline
\end{tabular}




\begin{tabular}{|l|l|}
\hline S. No. & $13-11-282$ \\
\hline Name & Shafiullah \\
\hline F/Name & Gul Rahman \\
\hline Age & 35 \\
\hline Sex & Male \\
\hline Address in Pakistan & Taimor Gara Camp, NWFP. Pakistan \\
\hline Address in Afghanistan & Jalalabad, Afghanistan \\
\hline Accusation/Crime & He was arrested under section 188. \\
\hline Date arrested & \\
\hline Prison & Dir (upper) \\
\hline Type of Prison & Sub Jail \\
\hline Present condition & Not released. \\
\hline Remarks & \\
\hline
\end{tabular}

\begin{tabular}{|l|l|}
\hline S. No. & $13-12-283$ \\
\hline Name & Nasruddin \\
\hline FiName & Jalaluddin \\
\hline Age & 35 \\
\hline Sex & Male \\
\hline Address in Pakistan & Taimor Gara, NWFP, Pakistan \\
\hline Address in Afghanistan & Kundoz, Afghanistan \\
\hline Accusation/Crime & He was arrested under section 14 Islamic Law. \\
\hline Date arrested & Dir (upper) \\
\hline Prison & Sub Jail \\
\hline Type of Prison & Not released. \\
\hline Present condition & \\
\hline Remarks &
\end{tabular}

\begin{tabular}{|c|c|}
\hline S. No. & $13-13-284$ \\
\hline Name & Salar \\
\hline F/Name & Mohammed \\
\hline Age & 35 \\
\hline Sex & Male \\
\hline Address in Pakistan & Balambat, NWFP, Pakistan \\
\hline Address in Afghanistan & Logar, Afghanistan \\
\hline Accusation/Crime & He was arrested under section $107 / 151$. \\
\hline \multicolumn{2}{|l|}{ Date arrested } \\
\hline Prison & Dir (upper) \\
\hline Type of Prison & Sub Jail \\
\hline Present condition & Not released. \\
\hline Remarks & \\
\hline
\end{tabular}




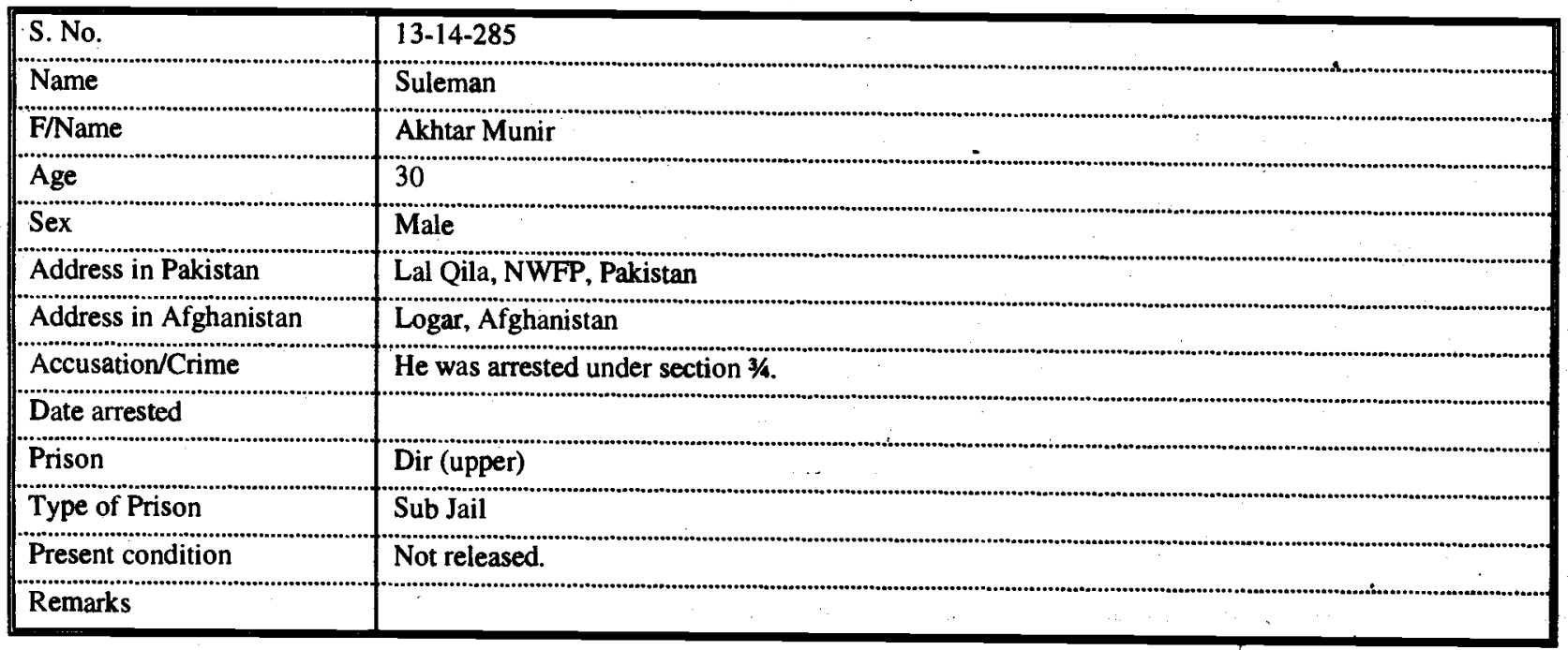

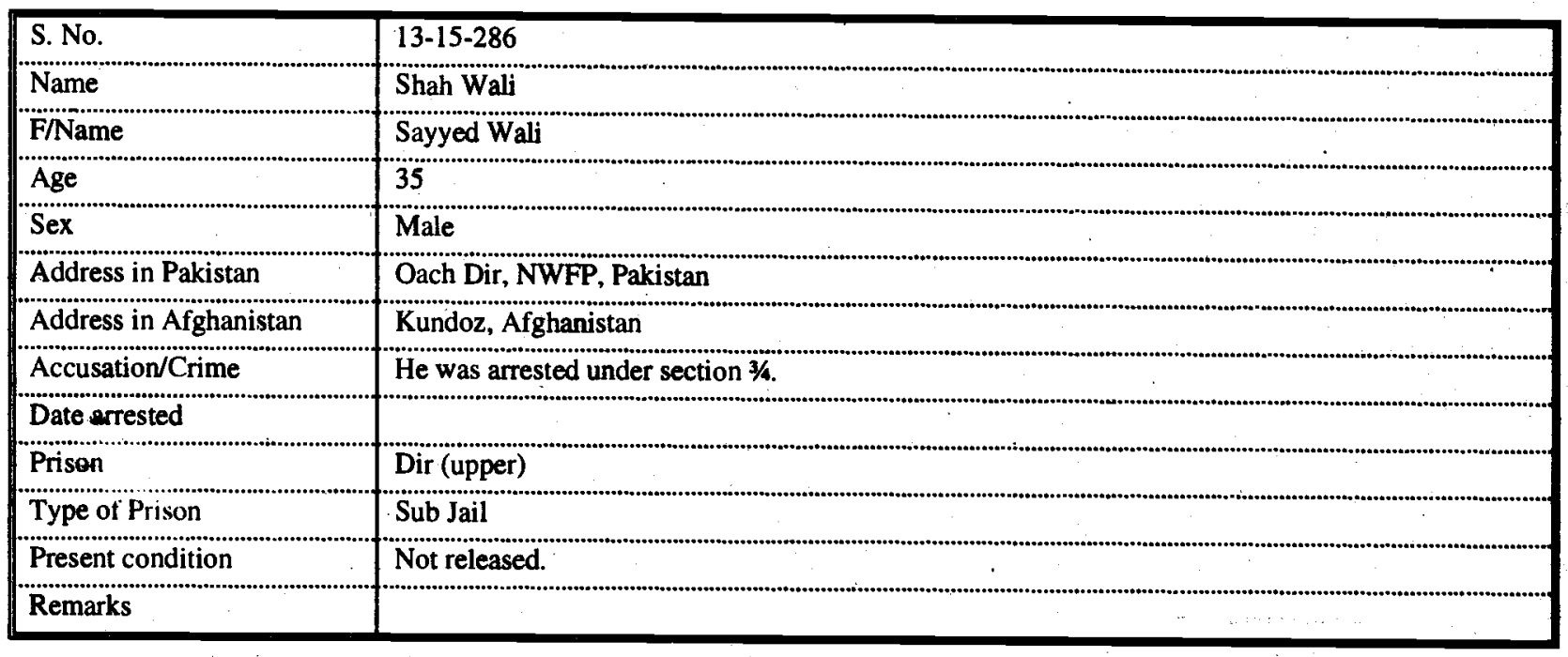

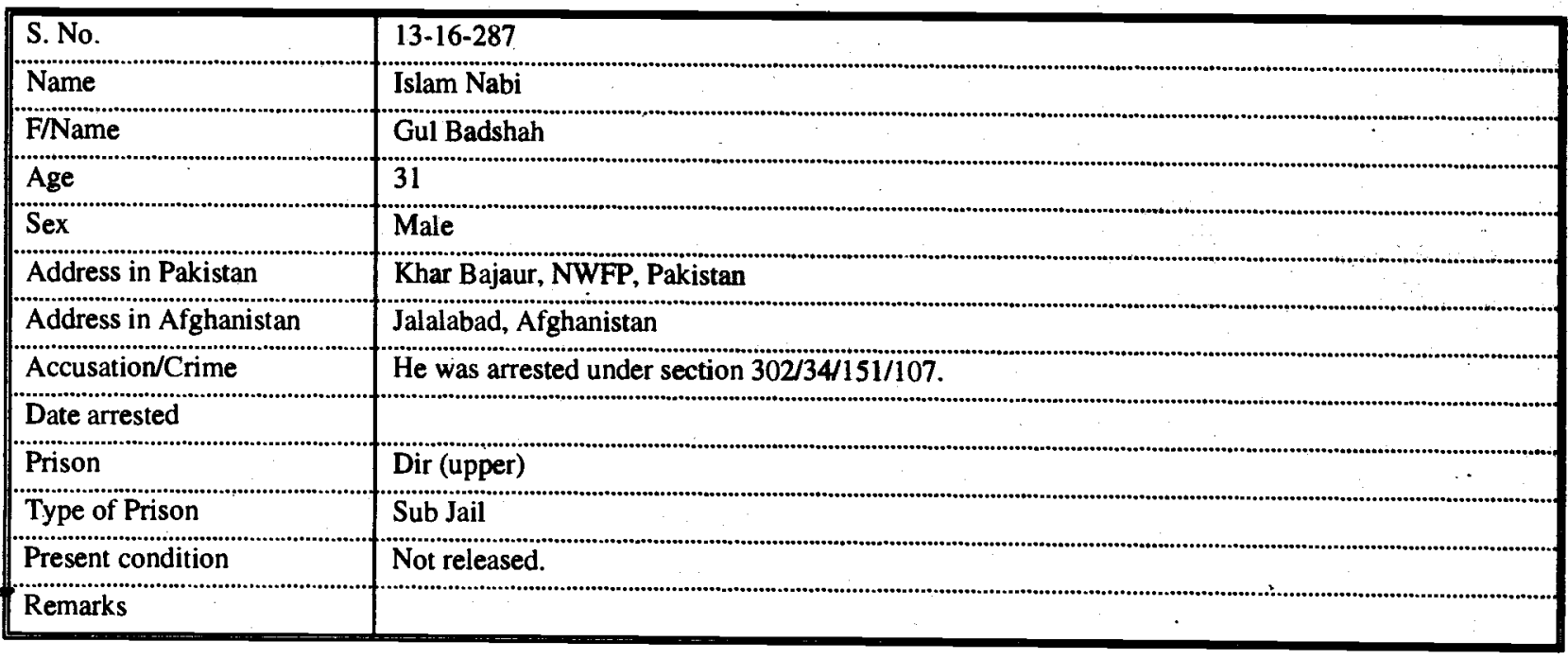




\begin{tabular}{|c|c|}
\hline S. No. & $13-17-288$ \\
\hline Name & Sarfaraz \\
\hline F/Name & Nadir Shah \\
\hline Age & 45 \\
\hline Sex & Male \\
\hline Address in Pakistan & Galgot Mayar Camp, NWFP, Pakistan \\
\hline Address in Afghanistan & Logar, Afghanistan \\
\hline Accusation/Crime & He was arrested under section $107 / 151$. \\
\hline \multicolumn{2}{|l|}{ Date arrested } \\
\hline Prison & Dir (upper) \\
\hline Type of Prison & Sub Jail \\
\hline Present condition & Not released. \\
\hline Remarks & \\
\hline
\end{tabular}

\begin{tabular}{|c|c|}
\hline S. No. & $13-18-289$ \\
\hline Name & Fazl Ghafar \\
\hline F/Name & Bakhtiyar \\
\hline Age & 40 \\
\hline Sex & Male \\
\hline Address in Pakistan & Chakdara Camp, NWFP, Pakistan \\
\hline Address in Afghanistan & Kunar, Afghanistan \\
\hline Accusation/Crime & He was arrested under section $302 / 34$. \\
\hline \multicolumn{2}{|l|}{ Date arrested } \\
\hline Prison & Dir (upper) \\
\hline Type of Prison & Sub Jail \\
\hline Present condition & Not released. \\
\hline Remarks & \\
\hline
\end{tabular}

\begin{tabular}{|l|l|l|}
\hline S. No. & $13-19-290$ \\
\hline Name & Jangir & Gul Pur \\
\hline FName & 25 \\
\hline Age & Male \\
\hline Sex & Chakdara Camp, NWFP, Pakistan \\
\hline Address in Pakistan & Kunar, Afghanistan \\
\hline Address in Afghanistan & He was arrested under section 14 Islamic law. \\
\hline Accusation/Crime & Dir (upper) \\
\hline Date arrested & Sub Jail \\
\hline Prison & Not released. \\
\hline Type of Prison & \\
\hline Present condition & \\
\hline Remarks &
\end{tabular}




\begin{tabular}{|c|c|}
\hline S. No. & $13-20-291$ \\
\hline Name & Khwidad \\
\hline F/Name & Amanullah \\
\hline Age & 25 \\
\hline Sex & Male \\
\hline Address in Pakistan & Oach Camp, NWFP, Pakistan \\
\hline Address in Afghanistan & \\
\hline Accusation/Crime & He was arrested under section 10/5/11. \\
\hline Date arrested & \\
\hline Prison & Dir (upper)" \\
\hline Type of Prison & Sub Jail \\
\hline Present condition & Not released. \\
\hline Remarks & \\
\hline
\end{tabular}

\begin{tabular}{|c|c|}
\hline S. No. & 1.3 .21 .292 \\
\hline Name & Baji Gul \\
\hline F/Name & Gul Rahman \\
\hline Age & 38 \\
\hline Sex & Male \\
\hline Address in Pakistan & Barawal Camp, NWFP, Pakistan \\
\hline Address in Afghanistan & Logar, Afghanistan \\
\hline Accusation/Crime & He was arrested under section $302 / 34$. \\
\hline Date arrested & \\
\hline Prison & Dir (upper) \\
\hline Type of Prison & Sub Jail \\
\hline Present condition & Not released. \\
\hline Remarks & \\
\hline
\end{tabular}

\begin{tabular}{|c|c|}
\hline S. No. & $13-22-293$ \\
\hline Name & Mushtaq \\
\hline F/Name & Tajbar Gul \\
\hline Age & 25 \\
\hline Sex & Male \\
\hline Address in Pakistan & Yusuf Abad Camp, NWFP, Pakistan \\
\hline Address in Afghanistan & Kunar, Afghanistan \\
\hline Accusation/Crime & He was arrested under section $5 / 10 / 11$ \\
\hline \multicolumn{2}{|l|}{ Date arrested } \\
\hline Prison & Dir (upper) \\
\hline Type of Prison & Sub Jail \\
\hline Present condition & Not released. \\
\hline Remarks & \\
\hline
\end{tabular}




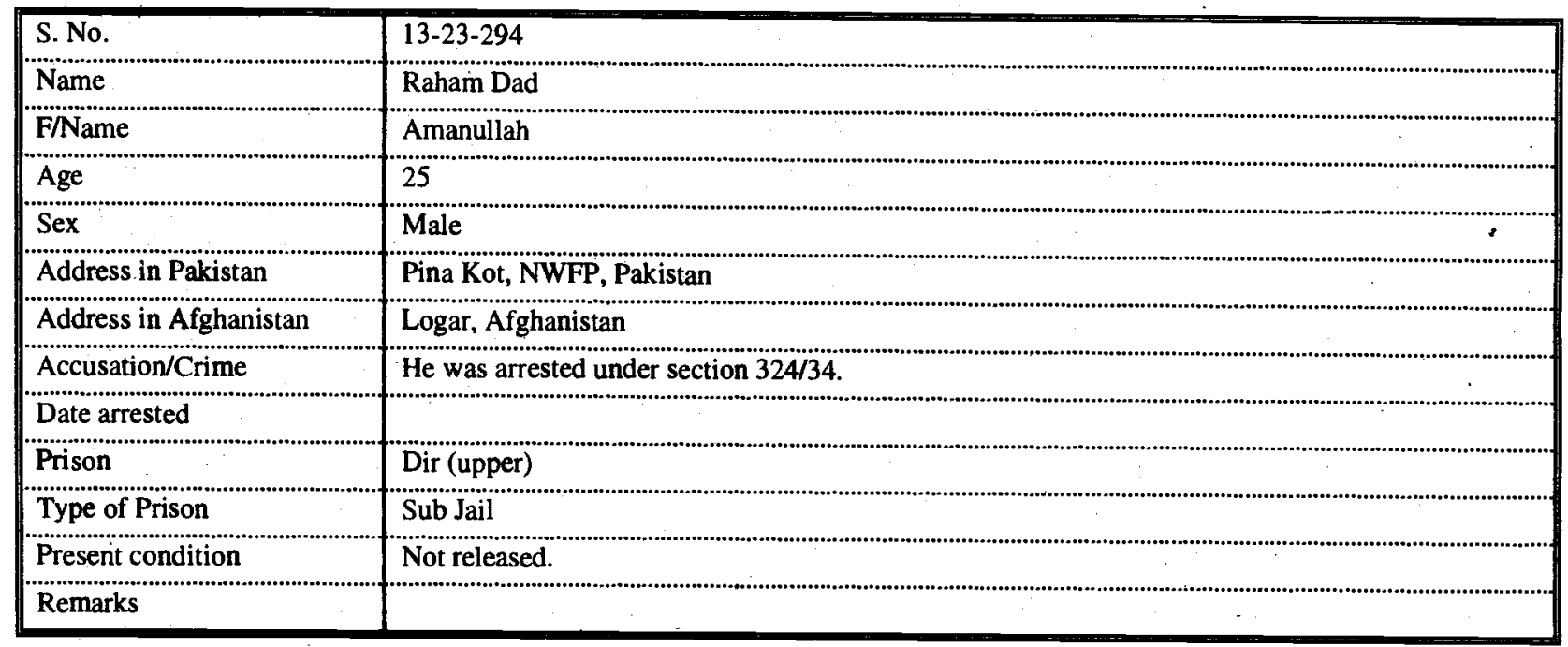




\section{Conclusions}

\section{Observations:}

1. Number of the surveyed prisons in NWFP (North West Frontier Provinces) was 10, among them. 2 were central prisons, 6 were district prisons and the remaining were judicial lockups.

2. 294 Afghan prisoners were found confined under different section of PPC Ex. P.A., Z.O., FCR and Islamic laws.

3. 88 were confined under 40 FCR (Frontier Crime Regulation) in district Prison Kohat and 11 were in central Prison Peshawar (Black Law for Tribal)

4. 10 juvenile delinquents ages 8 to 12 were found confined under section theft and $\mathrm{ZO}$ in different prisons.

5. 40 juvenile delinquents ages 13 to 21 were confined for the last two years.

6. It found that Afghan children were not treated as under trial i.e. should be innocent before being proven guilty.

7. 85 adult prisoners were confined under section 107, 109, 151, 4P.O. (Vagrancy act, and disobedient of Parents and drug addict).

8. 14 persons were confined under section 17(3) 14 Islamic law (theft charges).

9. 12 persons were under trail under section Z.O. 5/10/11 (Zina).

10. 11 persons were found in the prisons arrested under section $13 \mathrm{AO}$ (un licensed weapon).

11. 19 persons were found confined in different cases of abduction, murder dacoity and pick pocketing.

12. More than $44 \%$ had spent over 2 months of imprisonment while $34 \%$ had been in prisons more than two years.

13. $65 \%$ were not being provided legal aid by their own families, while $25 \%$ claimed to have legal aid by the parents and relative.

14. More than $75 \%$ did not get any visitors or got an occasional visit, so had to do without outside help.

15. $40 \%$ of the Afghan delinquent prisoners were students or had attended school earlier.

16. Afghan prisoners in NWFP: Prisons are a pathetic sight. They confronted legal human problems.

17. During the visit, Afghan juvenile delinquents approached us, these could not have been more than 10 years old, seared and bewildered, they said their parents did not know that they were in prisons. they were caught for stealing. Most of them were in bad condition. 
18. At district Prisons - Taimergara and Swat - Afghan prisoners complained us about maltreatment by the prisons staff.

19. All the Afghan prisoners complained us extraordinary delay in the hearing of their cases and non production in courts on the date of hearing.

20. The over-whelming number of Afghan prisoners in NWFP prisons have not been convicted of any crime. 20 convicted persons is different cases are confined in central prison Peshawar Haripur.

21. The excessive Afghan prisoners population in NWFP prisons in large due to deficiencies in the criminal justice process, both by the police and the courts.

22. $25 \%$ Afghan prisoners were complained about the violence in the police lockups during remand for the confessing of offence.

23. There is no special court for refugees in NWFP or even in Pakistan. The refugees and foreigners have been confined in prisons about 2 to 10 years.

24. UNHCR and other concerned agencies have not been contacted for help and releasing, which is most lamentable.

25. Local Afghan NGOs which are working in Pakistan or NWFP have not yet tried to help the oppressed prisoners in NWFP prisons nor have they proposed to the Govt. of Pakistan or other concerned authorities.

\section{Suggestions:}

1. United Nation and other agencies should introduce a legal aid fund to be located special for refugees/foreigners prisoners.

2. NGOs \& Human Rights Groups should be encouraged to specially monitor the cases of refugees $\&$ foreigners prisoners.

3. Those prisoners in Pakistan who have got no diplomatic agents of their states in Pakistan should be given facilities to concern with their states counsellors.

4. Refugees such as Afghanistan and others should be given facilities to communicate with UNGCR offices or any other international organisation.

5. Arrangements should be made for food, education, health, sanitation, residential, refreshment and environmental facilities according to the framed rules for foreigners or UN standard minimum rules for the treatment of prisoners.

6. Afghan NGOs, working in Pakistan have, the foremost duty to make a long terms program to work together for helping Afghan prisoners. They must have liaison with concerned authorities.

7. Committee, consisting on Afghan senior advocates, should be constituted for this purpose.

8. A legal aid cell should be established to provide the innocent prisoners with legal aid and make necessary contacts with Pakistani authorities for the release of prisoners. 


\section{Crimes Sections}

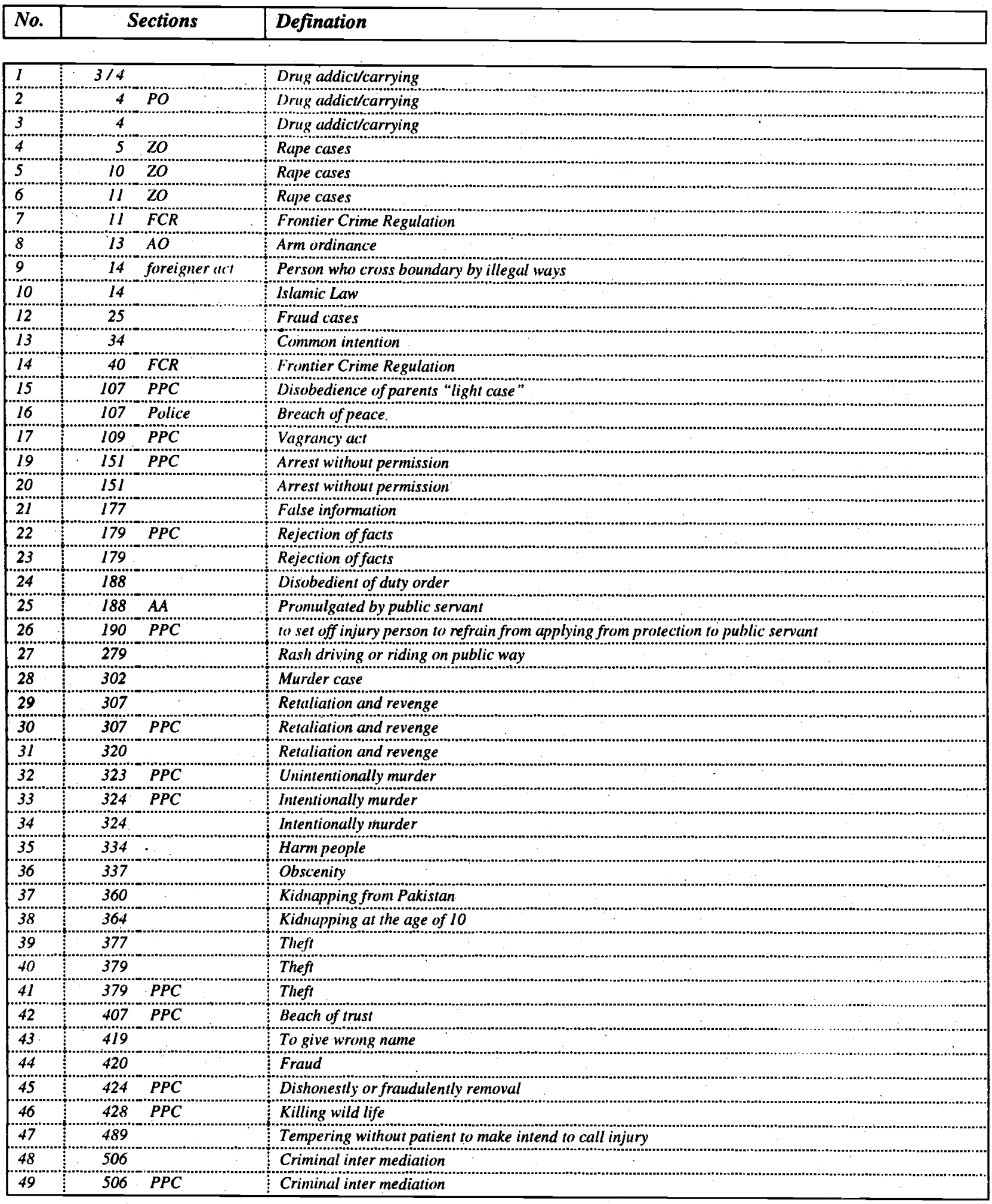


Q All human beings are born free and equal in dignity and rights. They ar endowed with reason and conscience and should act towards one another in a spirit of brotherhood.

2 No one shall be subjected to torture or to cruel, inhuman or degrading treatment or punishment.

Everyone has the right to recognition everywhere as a person before the law.

All are equal before the law and are entitled without any discrimination to equal protection $\mathrm{f}$ the law. All are entitled to equal protection against any discrimination in violation of this Declaration and against any incitement to such discrimination.

Everyone has the right to seek and to enjoy in other countries asylum from persecution.

This right may not be invoked in the case of prosecutions genuinely arising from non-political cremes or from acts contrary to the purposes and principles of the United Nations. 